\title{
EXPERIMENTAL STUDY OF PROFILE LOSSES IN THREE TRANSONIC TURBINE CASCADES
}

by

\author{
Christopher Sooriyakumaran
}

A thesis submitted to the Faculty of Graduate Studies and Postdoctoral Affairs in partial fulfilment of the requirements for the degree of

\section{Master of Applied Science}

in

Aerospace Engineering

\author{
Ottawa-Carleton Institute for \\ Mechanical \& Aerospace Engineering \\ Department of Mechanical and Aerospace Engineering \\ Carleton University \\ Ottawa, Ontario
}

(c) 2014

Christopher Sooriyakumaran 
The undersigned recommend to

the Faculty of Graduate Studies and Postdoctoral Affairs

acceptance of the thesis entitled

Experimental Study of Profile Losses in Three Transonic Turbine Cascades

submitted by

\section{Christopher Sooriyakumaran}

in partial fulfilment of the requirements for the degree of

Master of Applied Science in Aerospace Engineering

Thesis Supervisor:

Professor Steen A. Sjolander

Department of Mechanical \& Aerospace Engineering

Department Chair:

Professor Metin I. Yaras

Department of Mechanical \& Aerospace Engineering

Carleton University 
for Nora 


\section{Abstract}

This thesis documents the experimental study of the aerodynamic performance of transonic turbine cascades, with particular emphasis on the profile losses generated at design incidence. The midspan flow was measured for three transonic turbines from two Siemens industrial gas turbine engines. The measured profile losses were compared with the predictions from the Kacker \& Okapuu (1982) loss system. Loss predictions for a fourth turbine were also made.

The influence of outlet Mach number was investigated and measurements were obtained at Mach numbers between 0.8 and 1.4. Reynolds numbers were allowed to vary with the Mach number and values from $6.5 \times 10^{5}$ to $1.4 \times 10^{6}$ were observed. The influence of surface roughness on the correction for Reynolds number effects was examined. Finally, measurements were obtained at a number of different inlet flow angles ranging from $-30^{\circ}$ to $30^{\circ}$.

It was found that the loss predictions did not accurately capture trends observed in the data. Losses for the turbines were underpredicted, particularly those with negative inlet metal angles. This was attributed to the manner in which profile and trailing edge losses for these turbines are predicted from an extrapolation of empirical nozzle and impulse blade loss data in the Kacker \& Okapuu system. An alternative correction for Reynolds number effects due to Aungier (2006) was shown to slightly improve the loss predictions for a rough cascade. The measured losses also showed a tendency to level off in a plateau at low supersonic Mach

numbers. This is not predicted by the Kacker \& Okapuu loss system. It is suggested that the extent of the loss plateau may be influenced by the amount of unguided turning in the blade passage. 


\section{Acknowledgments}

This project has been one of learning, pride and determination. However, it has also been one of uncertainty and doubt. Without the overwhelming support and encouragement of a number of individuals, it would not have been possible.

I would first like to acknowledge the constant support and guidance of my thesis supervisor, Professor Steen Sjolander. His comprehensive knowledge of turbomachinery and deep understanding of experimental fluid mechanics proved invaluable to me throughout the course of this project. I would also like to humbly thank him for reviewing this thesis, which I am certain was no easy task. A number of colleagues also deserve recognition. Farzard Taremi, for instructing me in the art of diligent experimentation and patience. Steven Hall, for his guidance and shared experiences in the wind tunnel as well as for the countless discussions of aerodynamics and fantasy literature. Hamza Abo Ella, Andrew Scribner, Gordon MacIsaac, Jean-Michel Prevost, Sean MacTavish, Mitch Kibsey and Adrian Gerber were always available with insight and a helpful hand.

The financial and technical support of Siemens Energy, Inc. is gratefully acknowledged. Matthew Montgomery and Tobias Buchal of Siemens also offered very helpful direction and insight at every step of the experimental portion of this research project.

I owe thanks to Graham Beard and the other technicians of the Science and Technology Centre for their patience and willingness to work one-on-one to ensure the success of the project. The help provided from the faculty and staff of the Department of Mechanical and Aerospace engineering is also gratefully appreciated.

Finally, I wish to acknowledge the unyielding support and motivation from my parents and siblings, without whom this would never have been possible. 


\section{Table of Contents}

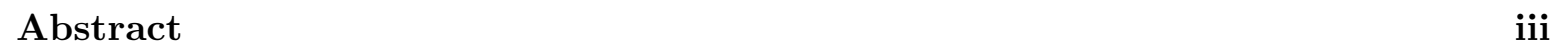

\begin{tabular}{ll}
\hline Acknowledgments & iv
\end{tabular}

List of Tables $\quad$ ix

List of Figures $\quad x$

Nomenclature xiii

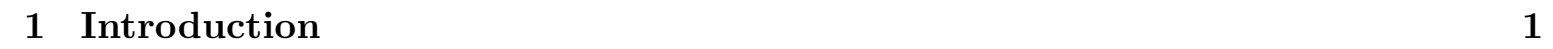

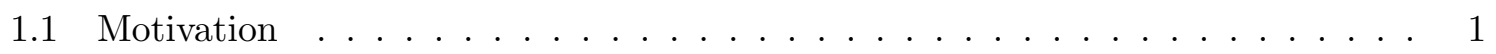

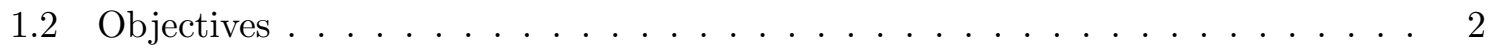

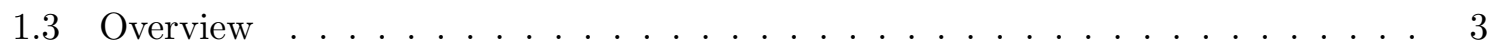

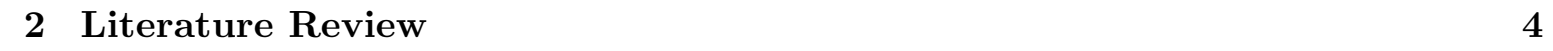

2.1 Introduction . . . . . . . . . . . . . . . . . . . . . 4

2.2 Flow Features and Loss Mechanisms in Axial Flow Turbines . . . . . . . . . . . 4

2.3 Loss Coefficient Definitions $\ldots \ldots \ldots \ldots \ldots \ldots$

2.4 Midspan Losses in Transonic Cascades . . . . . . . . . . . . . . . . . . . 9

2.5 Aerodynamic Influences on Midspan Losses . . . . . . . . . . . . . . . . . . . . 11

2.5 .1 Compressibility Effects . . . . . . . . . . . . . . . . 11

$2.5 .2 \quad$ Base Pressure . . . . . . . . . . . . . . . . . . . . . . . . 14

2.5 .3 Reynolds Number and Free Stream Turbulence Intensity . . . . . . . . . 15 
2.5 .4 Surface Roughness $\ldots \ldots \ldots \ldots \ldots \ldots$

$2.5 .5 \quad$ Effects of Off-Design Incidence $\ldots \ldots \ldots \ldots \ldots \ldots$

2.6 Correlation Systems for Predicting Profile Losses of Axial Turbines . . . . . . . 21

2.6 .1 Introduction . . . . . . . . . . . . . . . . . 21

2.6 .2 Ainley \& Mathieson Loss Correlation . . . . . . . . . . . . . . 21

2.6 .3 Modifications due to Dunham \& Came. . . . . . . . . . . . . . . . . 25

$2.6 .4 \quad$ Modifications due to Kacker \& Okapuu $\ldots \ldots \ldots \ldots \ldots \ldots$

2.6.5 Recent Modifications to the Ainley \& Mathieson Family of Loss Correlations . . . . . . . . . . . . . . . . . . . . . 31

2.6.6 Alternatives to the Ainley \& Mathieson Family of Loss Correlations . . 32

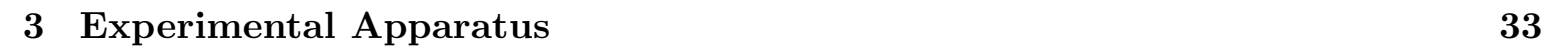

3.1 Introduction . . . . . . . . . . . . . . . . . . . 33

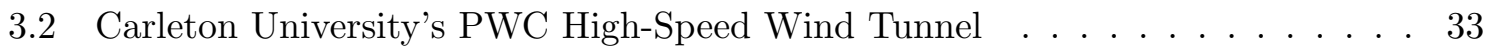

3.2 .1 Wind Tunnel Overview . . . . . . . . . . . . . . . . . 33

$3.2 .2 \quad$ Supply Air Delivery System $\ldots \ldots \ldots \ldots \ldots$

3.2 .3 Control and Shutoff Valves . . . . . . . . . . . . . . . . 37

3.2 .4 Test Section and Ejector-Diffusor Assembly . . . . . . . . . . . . 37

3.2 .5 Probe Traverse Mechanism . . . . . . . . . . . . . . . . 42

3.3 Pressure and Temperature Sensors $\ldots \ldots \ldots \ldots$. . . . . . . . . 43

3.4 Pressure Measurement Probes . . . . . . . . . . . . . . . . . . . . . . 44

3.4 .1 Three Hole Pressure Probe $\ldots \ldots \ldots$. . . . . . . . . . . . . 44

3.4 .2 Static Pressure Probe $\ldots \ldots \ldots \ldots \ldots$

$3.4 .3 \quad$ Endwall Boundary Layer Probes $\ldots \ldots \ldots \ldots$

$3.5 \quad$ Probe Calibration $\operatorname{Rig} \ldots \ldots \ldots \ldots \ldots$

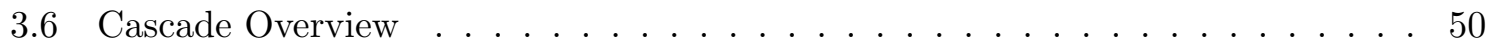

$3.6 .1 \quad$ Geometry $\ldots \ldots \ldots \ldots \ldots \ldots \ldots$

$3.6 .2 \quad$ Manufacturing $\ldots \ldots \ldots \ldots \ldots \ldots \ldots \ldots \ldots \ldots$

$3.6 .3 \quad$ Cascade Assembly $\ldots \ldots \ldots \ldots \ldots \ldots \ldots \ldots$ 
$\begin{array}{lll}4 & \text { Experimental Procedures and Measurement Uncertainties } & 56\end{array}$

4.1 Introduction . . . . . . . . . . . . . . . . . . . . . . . . . 56

4.2 Data Acquisition and Control System . . . . . . . . . . . . . . . . 57

4.2 .1 Measurement Locations . . . . . . . . . . . . . . . . . . 57

$4.2 .2 \quad$ Data Acquisition System . . . . . . . . . . . . . . . . . 59

4.2 .3 Data Sampling Rates and Integration Times . . . . . . . . . . . . . . . 60

4.2 .4 Control System . . . . . . . . . . . . . . . . . . . . . . . 61

4.3 Three-Hole Probe Calibration . . . . . . . . . . . . . . . . . . . . . . . . . 62

4.4 Data Reduction Procedures . . . . . . . . . . . . . . . . . . . . 66

4.4 .1 Three-Hole Probe Data Reduction . . . . . . . . . . . . . . . . 66

4.4 .2 Rayleigh Pitot Correction . . . . . . . . . . . . . . 66

$4.4 .3 \quad$ Alignment Procedure for Three-Hole and Static Pressure Probe Measurements . . . . . . . . . . . . . . . . 6 68

$4.4 .4 \quad$ Averaging and Mixing Calculations of Non-Uniform Flow . . . . . . . . 69

4.5 Measurement Uncertainty . . . . . . . . . . . . . . . . . . . . . . 72

4.6 Flow Quality . . . . . . . . . . . . . . . . . . . . . . . 74

$4.6 .1 \quad$ Introduction $\ldots \ldots \ldots \ldots \ldots \ldots \ldots$

4.6 .2 Inlet Endwall Boundary Layer . . . . . . . . . . . . . . . . 75

4.6 .3 Inlet Pitchwise Flow Uniformity $\ldots \ldots \ldots$. . . . . . . . 77

4.6 .4 Outlet Flow Periodicity $\ldots \ldots \ldots \ldots \ldots$. . . . . . . . . . . . . . . .

\begin{tabular}{lll}
\hline 5 & Experimental Results & 85
\end{tabular}

$5.1 \quad$ Introduction $\ldots \ldots \ldots \ldots \ldots \ldots \ldots \ldots \ldots$

5.2 Test Matrix . . . . . . . . . . . . . . . . . . . . . . 86

5.3 Blade Loadings for E2S50 f . . . . . . . . . . . . . . . . . . . 88

$5.4 \quad$ Experimentally Determining Design Incidence $\ldots \ldots \ldots$. . . . . . . . . . . . 95

5.5 Results for the Siemens Cascades Near Design Incidence . . . . . . . . . . . . . 99

$5.5 .1 \quad$ Profile Losses $\ldots \ldots \ldots$. . . . . . . . . . . . . . . . . . . . . . . . . . 99

5.5 .2 Outlet Flow Angle . . . . . . . . . . . . . . . . . . 105 
5.5 .3 Reynolds Number Variations . . . . . . . . . . . . . . . . . . . 110

$5.6 \quad$ Kacker \& Okapuu Prediction for Siemens Cascades . . . . . . . . . . . . . . . 110

5.7 Effects of Reynolds Number and Surface Roughness on Profile Loss Predictions 115

$5.7 .1 \quad$ Introduction $\ldots \ldots \ldots \ldots \ldots \ldots \ldots$

5.7.2 Alternatives to the Kacker \& Okapuu Reynolds Number Correction and the Effects of Surface Roughness . . . . . . . . . . . . . . . . 115

5.7 .3 Effect of Reynolds Correction on E2S50 Loss Prediction . . . . . . . . . 119

5.8 Future Work . . . . . . . . . . . . . . . . . . . . . . . . . . . . . . . . 121

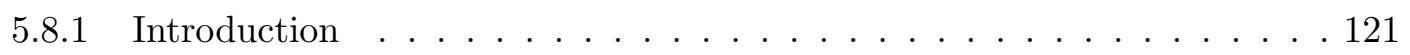

5.8.2 Effect of Negative Inlet Angles on Kacker \& Okapuu Profile Loss Prediction . . . . . . . . . . . . . . . . . . . 121

5.8.3 Effect of Negative Inlet Angles on Kacker \& Okapuu Trailing Edge Loss Prediction . . . . . . . . . . . . . . . . . . . . . 127

5.8 .4 Mach Number and Reynolds Number Corrections . . . . . . . . . . . . . 130

\begin{tabular}{|lll}
\hline 6 & Conclusions and Recommendations for Future Work & 132
\end{tabular}

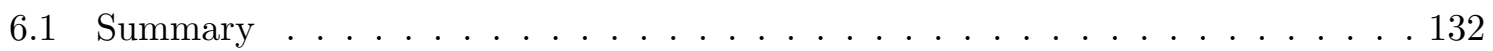

6.2 Conclusions . . . . . . . . . . . . . . . . . . . . . . 133

6.3 Recommendations for Future Work . . . . . . . . . . . . . . . . . . . . . . 134

\begin{tabular}{lr}
\hline List of References & 136
\end{tabular}

\begin{tabular}{|l|l|}
\hline Appendix A Siemens Cascade Geometry & 142
\end{tabular}

\begin{tabular}{|l|l|l|l|}
\hline Appendix B & Amecke \& Šafar̆ík & Method for Mixing-Out Non-Uniform Flows 150
\end{tabular}

\begin{tabular}{|ll|}
\hline Appendix C & Mixed-Out Results for the Siemens Cascades \\
\hline
\end{tabular} 


\section{List of Tables}

$3.1 \quad$ Valve Summary $\ldots \ldots \ldots \ldots \ldots$

3.2 Druck Transducer Summary . . . . . . . . . . . . . . . . . . . . . . 43

3.3 Cascade Geometry . . . . . . . . . . . . . . . . . . . 52

4.1 Summary of the Uncertainties Calculated by Corriveau[(2005)] . . . . . . . . . 74

$4.2 \quad$ Mixed-Out Periodicity $\ldots \ldots \ldots \ldots \ldots$. . . . . . . . . . . . . . . . 83

5.1 Wind Tunnel Operating Conditions . . . . . . . . . . . . . . . . 87

5.2 Estimated Design Incidence $\ldots \ldots \ldots$. . . . . . . . . . . . . . 99

A.1 Siemens Cascade Coordinates . . . . . . . . . . . . . . . . . . . . . . 142

A.1 Siemens Cascade Coordinates . . . . . . . . . . . . . . . . . 143

A.1 Siemens Cascade Coordinates . . . . . . . . . . . . . . . . . . . . 144

A.1 Siemens Cascade Coordinates . . . . . . . . . . . . . . . . . 145

A.1 Siemens Cascade Coordinates . . . . . . . . . . . . . . . . . 146

A.1 Siemens Cascade Coordinates . . . . . . . . . . . . . . . . . . . . . . 147

A.1 Siemens Cascade Coordinates . . . . . . . . . . . . . . . . . . . . . . . . 148

A.1 Siemens Cascade Coordinates . . . . . . . . . . . . . . . . . . . . . . . . . . . . 149

C.1 E1S50 Mixed-Out Results . . . . . . . . . . . . . . . . . . . . 154

C.1 E1S50 Mixed-Out Results . . . . . . . . . . . . . . . . . . . . . 155

C.2 E2S50 Mixed-Out Results . . . . . . . . . . . . . . . . . 156

C.3 E2S90 Mixed-Out Results . . . . . . . . . . . . . . . . . . . . . 157 


\section{List of Figures}

2.1 Flow Features in an Axial Flow Turbine Blade Passage (Adapted from Jeffries, 2000 by Corriveau, 2005) . . . . . . . . . . . . . . . . . 5

2.2 h-s Diagram for an Expansion Process with no Work or Heat Transfer (Adapted from Denton $[1993) \ldots \ldots \ldots \ldots \ldots \ldots$

$2.3 \quad$ Kinetic Energy Loss Coefficient Components at the Midspan of a Transonic Turbine Cascade (Mee et al. [1992)] . . . . . . . . . . . . . . . . . . . . 10

2.4 Shock Wave-Boundary Layer Interactions (Graham \& Kost, 1979) . . . . . . . 13

2.5 Trailing Edge Shock System (Denton \& Xu[ 1990) . . . . . . . . . . . . . . . 14

2.6 Influence of Reynolds Number on Turbine Cascade Performance (Hourmouziadis $[1989] \ldots \ldots \ldots \ldots \ldots$

$2.7 \quad$ Total Pressure Loss Coefficient Variation with Reynolds Number at $M_{2}=0.59$ (Ladwig \& Fottner 1993)] . . . . . . . . . . . . . . . . . 17

2.8 Total Pressure Loss Coefficient Variation with Reynolds Number at Different Turbulence Intensities (Hoheisel et al. [1987)] . . . . . . . . . . . . . . . . . . . 18

2.9 Loss Behaviour at Off-Design Incidence (Mayle, 1991)] . . . . . . . . . . . . . . 20

2.10 Profile Loss Coefficients at Design Incidence: $t_{\max } / c=0.2 ; R e=2 \times 10^{5}$; $M_{2}<0.6$ (Reproduced from Ainley \& Mathieson[ 1951)] . . . . . . . . . . . . 23

2.11 Correction Factor for Trailing Edge Thickness (Ainley \& Mathieson, 1951)] . . 24

2.12 Trailing Edge Energy Loss Coefficient (Reproduced from $\mid$ Kacker \& Okapuu,

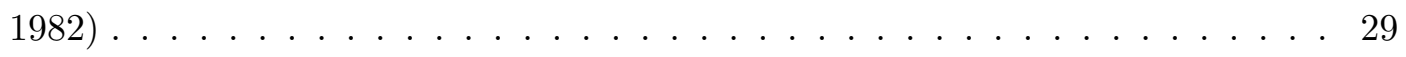

2.13 Comparison of Predicted Efficiency with Measured Efficiency of 33 Turbines (Kacker \& Okapuu, 1982)] . . . . . . . . . . . . . . . . . 30 
$3.1 \quad$ Pratt \& Whitney Canada High-Speed Wind Tunnel (Jeffries \& Sjolander, 1995) 34

3.2 Broom-Wade VC500 Compressor (Corriveau, 2005)] . . . . . . . . . . . . 36

3.3 Test Section (Corriveau $[2005)] \ldots \ldots \ldots \ldots \ldots$

3.4 Ejector-Diffusor Assembly (Jeffries \& Sjolander, 1995)] . . . . . . . . . . . . . 40

3.5 Test Section and Probe Traverse Mechanism (Jouini et al.[2002)] . . . . . . . . 41

3.6 Transducer Calibration Curves . . . . . . . . . . . . . . . . . . . . . . . . . . 44

3.7 Three Hole Probe $(\operatorname{Islam}[1999)] \ldots \ldots$. . . . . . . . . . . . 45

3.8 Static Pressure Probe $(\operatorname{Islam}[1999)] \ldots \ldots$. . . . . . . . . . . . 47

3.9 Boundary Layer Probes $($ Corriveau $[2005)] \ldots \ldots$. . . . . . . . . . . . 48

3.10 Probe Calibration Rig $($ Hall $[2012)] \ldots \ldots$. . . . . . . . . . . . 49

3.11 Symbols for Describing Airfoil Geometry . . . . . . . . . . . . . . . . 51

3.12 Siemens Cascade Profiles . . . . . . . . . . . . . . . . . . . . 53

3.13 Cascade Mounting: Endplate and Mounting Platform . . . . . . . . . . . 55

4.1 Measurement Locations . . . . . . . . . . . . . . . . . . . . . . . . . . . 58

4.2 Three-Hole Probe Nomenclature $\ldots \ldots \ldots$. . . . . . . . . . . . . 63

4.3 Thee-Hole Probe Calibration Curves . . . . . . . . . . . . . . . . . . . . . 65

4.4 Three Hole Probe Bow Shock $\ldots \ldots \ldots$. . . . . . . . . . . . . . . . 67

4.5 Effect of Poorly Aligned Static Pressure Measurements . . . . . . . . . . . . . . 69

4.6 Nomenclature used for Mixing Calculation . . . . . . . . . . . . . . 71

4.7 Variation of Uncertainty on Pressure Measurements (Corriveau, 2005)] . . . . 73

4.8 Inlet Spanwise Variation of Mach Number for E2S50 at $\alpha_{1}=9^{\circ} . . . .76$

4.9 Inlet Pitchwise Flow Uniformity for E2S50 Near Design Incidence . . . . . . . . 78

4.10 Total Pressure Loss Periodicity . . . . . . . . . . . . . . . . . 80

4.11 Outlet Flow Angle Periodicity . . . . . . . . . . . . . . . . . . . 81

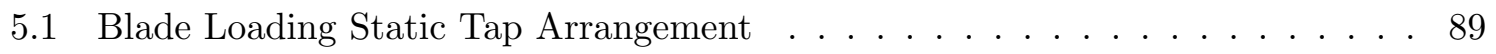

$5.2 \quad$ Surface Isentropic Mach Number $M_{s, i s}$ Distribution for E2S50 . . . . . . . . . . 90

$5.2 \quad$ Surface Isentropic Mach Number $M_{s, i s}$ Distribution for E2S50 (cont.) . . . . . 91

5.3 Trailing Edge Shock Structure.$\ldots \ldots$. . . . . . . . . . . . . . . 92

5.4 Blade Loadings for E2S50 . . . . . . . . . . . . . . . . . . 94 
5.5 Effect of Incidence on Losses for the Siemens Cascades . . . . . . . . . . . 96

$5.6 \quad$ Variation of Midspan Losses with Outlet Mach Number Near Design Incidence 100

5.7 Pitchwise Variation of Total Pressure Loss $C_{p o}$ for E1S50 $\ldots \ldots \ldots$

5.8 Pitchwise Variation of Total Pressure Loss $C_{p o}$ for E2S50 $\ldots \ldots \ldots$

5.9 Pitchwise Variation of Total Pressure Loss $C_{p o}$ for E2S90 $\ldots \ldots \ldots$

5.10 Variation of Outlet Flow Angle $\alpha_{2}$ with Mach Number Near Design Incidence . 106

5.11 Schematic of Probe Tip and Flow Angles $\ldots \ldots$. . . . . . . . . 107

5.12 Pitchwise Variation of Downstream Static Pressure for E1S50 Obtained with the Static Pressure Probe . . . . . . . . . . . . . . . . . . . 108

5.13 Variation of Outlet Reynolds Number $R e_{c}$ with Mach number Near Design Incidence . . . . . . . . . . . . . . . . . . . . . . 111

5.14 Kacker \& Okapuu Predicted Losses for Siemens Cascades . . . . . . . . . . . 113

5.15 Reynolds Corrections for Rough Cascades due to Kacker \& Okapuu (1982) and Aungier (2006) . . . . . . . . . . . . . . . . . . . . . . 118

5.16 Predicted loss for E2S50: Effect of Reynolds Correction . . . . . . . . . . 120

5.17 Loss Prediction at Negative Inlet Angles . . . . . . . . . . . . . . . . 123

5.18 Ratio of $Y_{P I}$ to $Y_{P N}$ from AMDC Correlation . . . . . . . . . . . 124

5.19 Kacker \& Okapuu Predicted Profile Losses for Siemens Cascade Families . . . . 125

5.20 Effect of $\frac{\beta_{1}}{\alpha_{2}}$ on $K_{t} \ldots \ldots \ldots \ldots \ldots \ldots \ldots \ldots \ldots \ldots \ldots \ldots \ldots \ldots \ldots$

5.21 Effect of $\frac{\beta_{1}}{\alpha_{2}}$ on $\Delta \phi_{T E T}^{2} \ldots \ldots \ldots \ldots \ldots \ldots \ldots \ldots \ldots \ldots \ldots \ldots \ldots \ldots \ldots$ 


\section{Nomenclature}

\section{Symbols}

$a$

$A V D R$

$b$

C

$C_{\alpha}$

$c_{f}$

$C_{o}$

$c_{p}$

$C_{p_{b}}$

$C_{p o}$

$C_{s}$

$c_{v}$

$e$

$f(R e)$

$h$ speed of sound

axial velocity density ratio $=\frac{\int_{0}^{1}\left(\rho_{2} V_{x, 2}\right)_{M S} d(y / s)}{\int_{0}^{1}\left(\rho_{1} V_{x, 1}\right)_{M S} d(y / s)}$

zero pressure voltage of pressure transducer

chord length (defined in Figure 3.11

three-hole probe calibration constant $=\frac{P_{\text {port } 2}-P_{\text {port } 3}}{P_{\text {port } 1}-\bar{P}}$

skin friction coefficient

three-hole probe calibration constant $=\frac{P_{o}-P_{\text {port } 1}}{P_{\text {port } 1}-\bar{P}}$

specific heat capacity at constant pressure

base pressure coefficient $=\frac{P_{b}-P_{2}}{P_{o 2}-P_{2}}$

total pressure loss coefficient $=\frac{P_{o 2}-P_{o 1}}{P_{o 2}-P_{2}}$

three-hole probe calibration constant $=\frac{P_{o}-P_{s}}{P_{\text {port } 1}-\bar{P}}$

specific heat capacity at constant volume

height of roughness elements, suction surface curvature

correction factor for Reynolds number effects

enthalpy, blade height 
$H$

$k_{1}, k_{2}, k_{p}$

$k_{c}$

$k_{t}$

$m$

$\dot{m}$

$N$

$O$

$P$

$P_{b}$

$P_{o}$

$q$

$R$

$R e_{c}$

$R e_{r}$

$S$

$S$

$t$ boundary layer shape factor $=\frac{\delta^{*}}{\theta}$

incidence angle (defined in Figure 3.11

subsonic compressibility correction for loss correlations

supersonic compressibility correction for loss correlations

thickness correction for loss correlations

transducer calibration curve slope

mass flow rate

number of blades in cascade

throat opening width (defined in Figure 3.11 ,

static pressure

base pressure

total (stagnation) pressure

dynamic pressure

specific gas constant

Reynolds number based on chord length $=\frac{\rho V c}{\mu}$

critical roughness Reynolds number $=100 c / e$

pitch spacing (defined in Figure 3.11 , specific entropy

entropy

trailing edge thickness (defined in Figure 3.11) 


\begin{tabular}{|c|c|}
\hline$T$ & temperature \\
\hline$t u$ & free stream turbulence intensity \\
\hline$u$ & streamwise velocity inside the boundary layer \\
\hline$U_{e}$ & streamwise velocity at boundary layer edge \\
\hline$V$ & velocity, voltage \\
\hline$w$ & distance between bounding streamlines \\
\hline$W$ & inlet duct width \\
\hline$W_{e}$ & wedge angle (defined in Figure 3.11 ) \\
\hline$x$ & axial direction \\
\hline$y$ & tangential (pitchwise) direction \\
\hline$Y$ & total pressure loss coefficient $=\frac{P_{o 1}-P_{o 2}}{P_{o 2}-P_{2}}$ \\
\hline$Y_{P}$ & profile loss coefficient \\
\hline$Y_{S}$ & secondary loss coefficient \\
\hline$Y_{S H O C K}$ & shock loss coefficient \\
\hline$Y_{T C}$ & tip clearance loss coefficient \\
\hline$Y_{T E T}$ & correction factor for trailing edge losses \\
\hline$Y_{T E T}^{\prime}$ & trailing edge loss coefficient \\
\hline$z$ & spanwise direction \\
\hline
\end{tabular}




\section{Greek Symbols}

$\alpha$

$\beta_{e}$

$\gamma$

$\delta$

$\delta^{*}$

$\delta_{99 \%}$

$\zeta$

$\eta$

$\theta$

$\theta_{U G}$

$\Theta$

$\mu$

$\xi$

$\rho$

$\sigma$

$\phi$ flow angle (defined in Figure 3.11

blade metal angle (defined in Figure 3.11), probe bevel angle (defined in Figure 4.2 )

probe asymmetry angle (defined in Figure 4.2

ratio of specific heats $=\frac{c_{p}}{c_{v}}$

deviation angle $=\beta_{2}-\alpha_{2}$

boundary layer displacement thickness (defined in Equation 4.18

boundary thickness

stagger angle (defined in Figure 3.11

efficiency

boundary layer momentum thickness (defined in Equation 4.18), calibration jet alignment angle (defined in Figure 4.2

unguided turning angle (defined in Figure 3.11

non-dimensional mass flow rate $=\frac{\rho V}{\rho^{*} a^{*}}$

dynamic viscosity

energy (or enthalpy) loss coefficient $=\frac{h_{2}-h_{2 s}}{h_{o 2}-h_{2}}$

density

standard deviation

fundamental quantity 

$\Delta \phi^{2}$
kinetic energy loss coefficient $=1-\frac{V_{2}^{2}}{V_{2 s}^{2}}$
$\Delta \phi_{T E T}^{2}$
trailing edge energy loss coefficient

\section{Subscripts}

\begin{tabular}{|c|c|}
\hline $0, o$ & total (stagnation) \\
\hline 1 & value at cascade inlet \\
\hline 2 & mixed-out value at cascade outlet \\
\hline $2 s, 2 i s$ & isentropic value at cascade outlet \\
\hline $2 y$ & measured value at cascade outlet \\
\hline$A$ & area-averaged value \\
\hline atm & atmospheric condition \\
\hline des & design \\
\hline$e, \delta$ & value at boundary layer edge \\
\hline$i s, s$ & isentropic value \\
\hline le & leading edge \\
\hline$\dot{m}$ & mass-averaged value \\
\hline $\max$ & maximum \\
\hline$M S$ & value at midspan \\
\hline$r e f$ & reference \\
\hline te, $T E T$ & trailing edge \\
\hline$r$ & axial \\
\hline
\end{tabular}


Abbreviations

$\mathrm{AM}$

AMDC

DMI

DMLS

HSWT

$\mathrm{KO}$

NI

NJ

PID

PS

PWC

SS

THP
Ainley \& Mathieson

Ainley \& Mathieson and Dunham \& Came

Directed MFG, Inc.

direct metal laser sintering

high-speed wind tunnel

Kacker \& Okapuu

National Instruments

Neles-Jamesbury

proportional-integral-derivative

pressure side, pressure surface

Pratt \& Whitney Canada

suction side, suction surface

three-hole probe 


\section{Chapter 1}

\section{Introduction}

\subsection{Motivation}

Although renewable and nuclear energy sources are gaining in importance, fossil fuels continue to be a major source of global power generation. Growing concerns of the environmental impact of power generation based on fossil fuels, the rising cost of fuel, and the rapidly increasing global energy needs have pushed gas turbine manufacturers to continually develop more cost effective, efficient and clean engines. To achieve these goals, gas turbine engine manufacturers optimize every aspect of the engines, including the turbine. To increase efficiency, turbine designers aim to reduce the pressure loss through each stage of the turbine. Although turbine efficiencies are high, gains can still be made through better understanding of the turbine aerodynamics. However, as engines become larger and the pressure ratios increase, the flow through the turbine often reaches supersonic conditions. Therefore, the study of turbine performance must include high-speed investigations.

The starting point for the aerodynamic design of turbines is typically a meanline analysis. Early stages of design consider only the flow at the mean radius and treat the flow as onedimensional. Many important decisions are made during the meanline analysis, some of which are very difficult to change at later stages of design. The success of meanline analysis

depends very heavily on the ability to accurately predict the losses of the turbine being designed. Therefore, a great deal of effort has been spent to develop accurate loss prediction systems that are built on empirical correlations. These correlations are typically developed 
using data for turbine losses obtained in cascades or rotating test rigs. Cascade testing in particular provides a simplified environment in which a great deal of insight into the flow physics of a turbomachinery blade row can be gained with relative ease when compared to rotating rigs or full engine tests. As the understanding of the flow physics improves and design technologies evolve, the loss correlations must be periodically updated and improved to reflect these advances and to allow for better optimization of the turbines.

As the name suggests, the main objective of meanline analysis is to determine turbine characteristics on the meanline. Consequently, much of the empirical loss data, on which the loss correlations are built, were obtained from turbine profiles typical of the midspan of the blade. However, due to their simplicity and effectiveness, the correlations developed for meanline analysis are sometimes applied across the full blade span. This is often referred to as throughflow analysis and represents a two-dimensional approximation to the flow in the machine. Blade geometry and flow conditions vary significantly across the span of the blade. Consequently, the correlations are extrapolated to predict the losses of turbine profiles which may fall outside the range of the empirical midspan data. In an effort to understand what implications this might have for the profile loss predictions of transonic turbines (profile losses being those generated in the boundary layers of the turbine), Siemens Energy, Inc. have funded the present investigation. The findings of the study are to be used to improve the meanline and throughflow analysis for Siemens engines.

\subsection{Objectives}

The turbomachinery research group at Carleton University has investigated the losses in turbines extensively over the past decades including a number of studies on the midspan (or profile) losses of high-speed cascades. This thesis builds heavily on the work of the researchers who have established a reliable facility for turbine aerodynamics research at Carleton. The wind tunnel used for the experimental measurements is the Pratt \& Whitney Canada highspeed wind tunnel, which is an intermittent blow-down wind tunnel that operates at transonic Mach numbers up to about 1.4 . 
The objectives of the current study is to investigate the profile losses of transonic turbine cascades and compare the experimental measurements with the predictions from the loss system developed by Kacker \& Okapuu (1982). Four turbine blade geometries were provided for the study, all of which were designed by Siemens. Due to time constraints, the profile losses were measured for only three of the cascades. However, the predicted profile losses of all four turbines are examined. This investigation is part of an effort to update and improve the loss prediction system used for the meanline analysis by turbine designers.

The thesis investigates the influence of Mach number and inlet flow angle on the losses, both measured and predicted. In addition, some discussion on the effects of surface roughness and Reynolds number is included.

\subsection{Overview}

Chapter 2 is a review of the open literature that is most relevant to the current study. Chapter 3 follows with descriptions of the experimental apparatus. The measurement procedures and uncertainties, as well as the data reduction techniques are described in Chapter 4 . The flow quality assessment is also discussed in Chapter 4. The midspan flow measurements obtained for the three Siemens cascades are discussed and compared with the empirical loss predictions in Chapter 5. Chapter 5 also indicates several areas of possible future research. Finally, the conclusions of the current study and a summary the recommendations for future work are given in Chapter 6 . 


\section{Chapter 2}

\section{Literature Review}

\subsection{Introduction}

The current study is an investigation of the performance of four transonic turbine cascades, with the aim to compare loss measurements with the loss predictions using methods described in the open literature. This chapter describes these prediction methods. However, the chapter opens with a description of the physical flow structures of a turbine blade row and the key mechanisms by which losses are generated. Profile losses in particular will be the focus of discussion. Following this, several aerodynamic influences on losses, such as Mach number and Reynolds number, will be discussed. Finally, the widely-used loss prediction system due to Ainley \& Mathieson (1951) and the subsequent improvements made to it will be described. Several less widely-used systems will also be mentioned briefly.

\subsection{Flow Features and Loss Mechanisms in Axial Flow Tur- bines}

The focus of this thesis is on the losses generated in the region of two-dimensional flow at the midspan of a turbine blade in a non-rotating linear cascade. Although cascade flows are simplified when compared to rotating annular turbine blade rows, it is worthwhile to first examine the general case of flow in turbine passage. Furthermore, the passage flow as a whole should be examined. The main flow features typical of a turbine passage are shown 


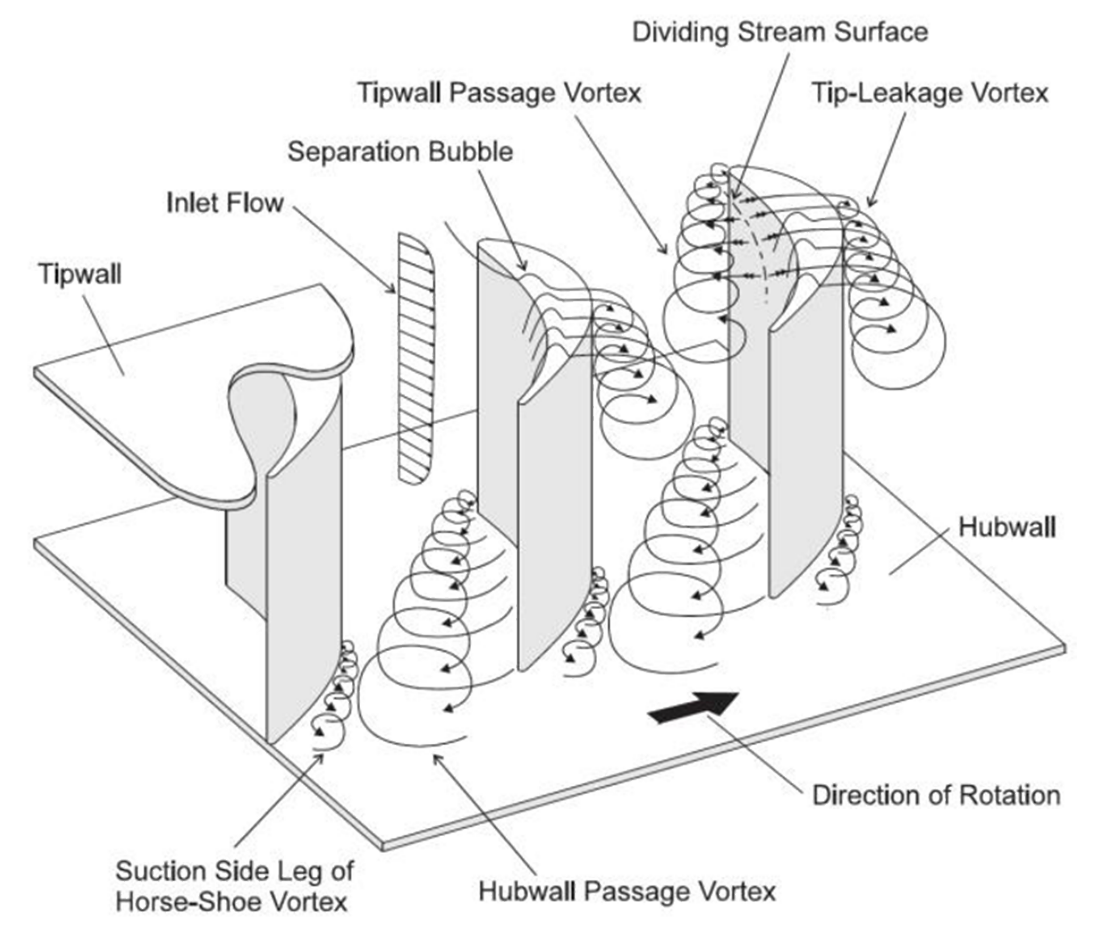

Figure 2.1: Flow Features in an Axial Flow Turbine Blade Passage (Adapted from Jeffries, 2000 by Corriveau, 2005)

schematically in Figure 2.1.

The inlet boundary layer that forms on the endwall upstream of the cascade separates around the leading edge of the turbine, forming two counter rotating vortices; together these are known as the horseshoe vortex. Due to the cross-passage pressure gradient that exists between the suction and pressure surfaces of adjacent blades, the pressure side leg of the horseshoe vortex is swept across the passage. The hubwall boundary layer within the blade passage is also affected by the cross-passage pressure gradient and it too rolls up into a vortex that combines with the pressure side leg of the horseshoe vortex. The resultant is called the passage vortex. These vortices will form at both the hub and the tip endwall. However, for rotor blades with tip clearance (as shown in Figure 2.1), some amount of tip leakage is typically present. Fluid will flow from the pressure side, over the tip of the blade, where there is often a separation bubble, and emerge on the suction side where it rolls up into a tipleakage vortex. Boundary layers form on the pressure and suction sides of the blade. Near the endwalls, these boundary layers are effected by the secondary flow structures. If the aspect 
ratio of the blade is sufficiently large, the flow at the meanline of the blade is two-dimensional. The surface boundary layers are sensitive to the blade loading (i.e. pressure distribution) of the airfoil. Under certain conditions, separation bubbles can form on either side of the blade. At transonic conditions, shock waves and Prandtl-Meyer expansion fans can form within the blade passage and at the trailing edge.

Entropy generation, or losses, in a turbine blade row can be influenced by many factors. Therefore, the losses are typically broken down into components. Mee et al. (1992) and Denton (1993) offer detailed examinations of these components. The typical loss break down is as follows:

Profile Losses are losses generated in the blade surface boundary layers. Losses here are dependent upon the state of the boundary layers (laminar or turbulent) and the free stream velocity. The interaction between the boundary layers and shock waves influences the profile loss production. Shock wave-boundary layer interaction is discussed further later in the chapter.

Trailing Edge Losses arise from the sudden expansion in flow area at the trailing edge of the blade. At the trailing edge, the blade surface boundary layers separate and a region of low pressure (called the base pressure) forms. This region is often associated with complex shock structures at transonic Mach numbers.

Shock Losses are caused by the entropy rise across shock waves in the free stream of the flow passage. These are limited to transonic cascades. Shock losses and trailing edge losses are sometimes considered part of the profile losses, in which case the term midspan loss is often used interchangeably with profile loss.

Secondary Losses are losses produced by the secondary flow structures. These may include the passage vortex and the suction side leg of the horseshoe vortex. Losses produced in the annulus boundary layer within the flow passage, and in the mixing of the secondary flow structures as they convect downstream, also contribute to the secondary losses.

Tip-Leakage Losses are the losses caused by tip-leakage flow in rotor blades. Viscous losses 
in the tip gap, mixing of the tip flow with the passage flow and the resulting tip-leakage vortex, all contribute to the tip-leakage losses.

Annulus Losses are the losses produced in the annulus boundary layers outside of the blade passage (i.e. between blade rows).

\subsection{Loss Coefficient Definitions}

Fundamentally, loss and inefficiency is the generation of entropy within the flow passage, i.e. the "loss" of potentially useful work. Entropy, however, is not a directly measurable quantity. This makes it difficult to determine changes in entropy experimentally. Thus, loss coefficients are introduced. These non-dimensional parameters allow for easy comparison between the losses of different turbines. There are many loss coefficients in use for characterising the losses of turbines; several will be discussed. For a detailed description of the loss coefficients see Denton (1993).

Figure 2.2 shows the enthalpy-entropy $(h-s)$ diagram for the expansion process in a turbine nozzle. Examining Figure 2.2, it can be seen that entropy generation, $\Delta s$ manifests itself in at least three ways: a decrease in total pressure between the initial (1) and final (2) states, an increase in static enthalpy at the final state, and a decrease in velocity at the final state.

Entropy can be expressed as a function of temperature $T$ and pressure $P$. For an ideal gas, the change in entropy in a process is,

$$
d S=c_{p} \frac{d T}{T}-R \frac{d P}{P}
$$

where $c_{p}$ is the specific heat at constant pressure and $R$ the gas constant. Assuming constant $c_{p}$ for an arbitrary process between two states, like that shown in Figure 2.2, the expression for the change in entropy can be written as,

$$
\Delta S=c_{p} \ln \left(\frac{T_{2}}{T_{1}}\right)-R \ln \left(\frac{P_{2}}{P_{1}}\right)
$$

Equations 2.1 and 2.2 are valid when using either static, or stagnation properties since, by definition, the changes from static to stagnation conditions are isentropic. 


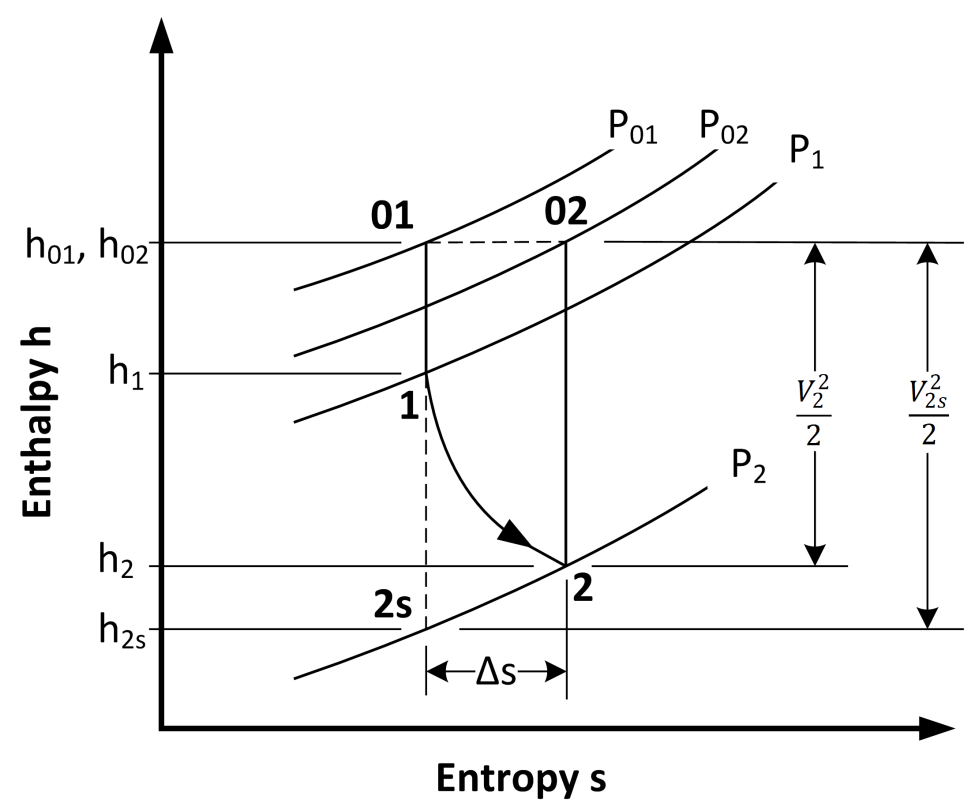

Figure 2.2: h-s Diagram for an Expansion Process with no Work or Heat Transfer (Adapted from Denton, 1993)

For linear cascade testing, no work is done on the fluid as it passes through the blade passage. Consequently, the total temperature $T_{o}$ is constant. Therefore, by Equation 2.2, the entropy rise is a function of only the total pressure drop, $\Delta P_{o}$ in the blade row. Conveniently, $\Delta P_{o}$ is easy to measure experimentally. This leads to the first, and perhaps most common loss coefficient: the total pressure loss coefficient, $Y$. For a turbine, $Y$ is defined as,

$$
Y=\frac{P_{o 1}-P_{o 2}}{P_{o 2}-P_{2}}
$$

where $P_{o 1}$ and $P_{o 2}$ are the mass-averaged or mixed-out values of the total pressure at the inlet and the outlet of the cascade respectively and $P_{2}$ is the static pressure at the outlet. The total pressure loss coefficient is frequently used for cascade measurements and several of the loss estimation systems use this definition. It will serve as the primary coefficient for the discussion of losses in this thesis.

The energy loss coefficient, $\xi$ is based on the enthalpy, $h$. It is also referred to as the total enthalpy loss coefficient. For a turbine, $\xi$ is defined by Denton (1993) as,

$$
\xi=\frac{h_{2}-h_{2 s}}{h_{o 2}-h_{2}}
$$


where $h_{2 s}$ is the static enthalpy that would have been obtained if the expansion to $P_{2}$ was isentropic. Since the slope of the constant pressure line, $P_{2}$ (i.e. the static temperature, $T_{2}$ ) is not constant, $\xi$ is only an approximation of the entropy generation. However, for processes in which $T_{2}-T_{2 s}$ is small, it may be a reasonable approximation. Other authors such as Kacker \& Okapuu (1982) and Mee et al. (1992), use a slightly different expression for the energy loss coefficient, which will be represented by $\Delta \phi^{2}$. It is defined as;

$$
\Delta \phi^{2}=\frac{h_{2}-h_{2 s}}{h_{o 2}-h_{2 s}}=1-\frac{V_{2}^{2}}{V_{2 s}^{2}}
$$

where $V$ is the velocity, which can be related to enthalpy as shown in Figure 2.2. Physically, the energy loss coefficient represents a deficiency in resulting velocity between the isentropic and the actual expansion process. Consequently, it is often called the kinetic energy loss coefficient. $\Delta \phi^{2}$ is used by Kacker \& Okapuu for the trailing edge loss component in the loss system. For incompressible flow, $\Delta \phi^{2}$ is related to the energy loss coefficient of Denton by,

$$
\xi=\frac{\Delta \phi^{2}}{1-\Delta \phi^{2}}
$$

and to the total pressure loss coefficient, $Y$ by,

$$
Y=\frac{\Delta \phi^{2}}{1-\Delta \phi^{2}}
$$

In general, $Y$ and $\Delta \phi^{2}$ are related by the following expression:

$$
Y=\frac{\left[1-\frac{\gamma-1}{2} M_{2}^{2}\left(\frac{1}{1-\Delta \phi^{2}}-1\right)\right]^{-\frac{\gamma}{\gamma-1}}-1}{1-\left(1+\frac{\gamma-1}{2} M_{2}^{2}\right)^{-\frac{\gamma}{\gamma-1}}}
$$

\subsection{Midspan Losses in Transonic Cascades}

For sufficiently high aspect ratio blades, the midspan losses are comprised of three components: the profile (boundary layer) losses; the trailing edge losses; and the shock losses.

Mee et al. (1992) experimentally examined the individual components of the midspan losses in a transonic cascade using detailed measurements in the boundary layer at the trailing edge, and measurements of the wake downstream of the cascade. The resulting breakdown of losses is shown in Figure 2.3 . 


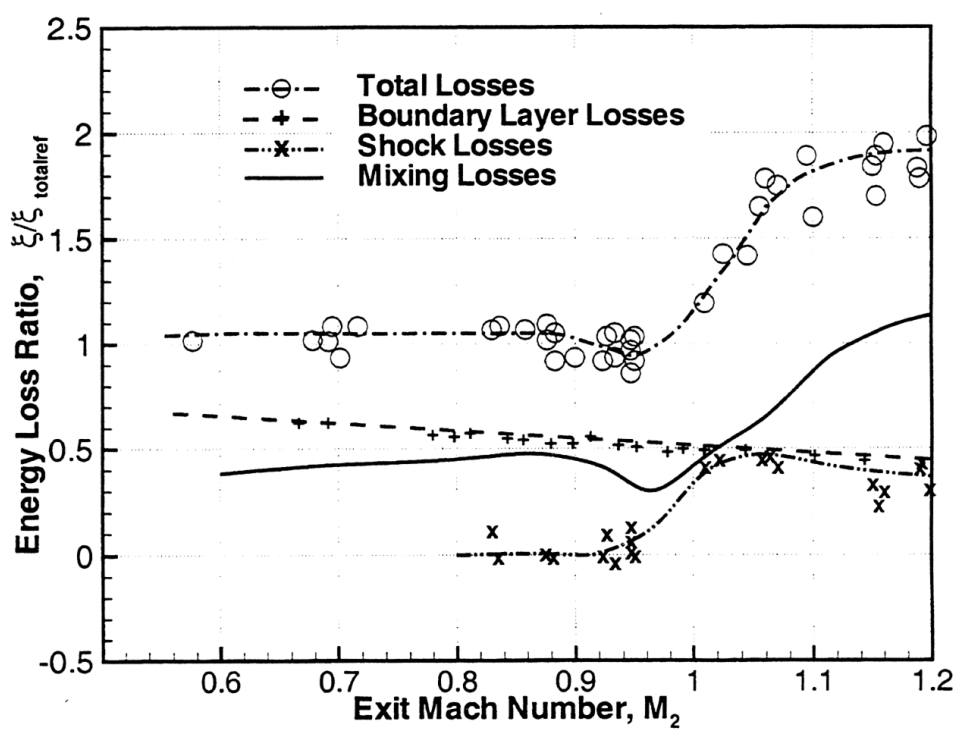

Figure 2.3: Kinetic Energy Loss Coefficient Components at the Midspan of a Transonic Turbine Cascade (Mee et al., 1992)

The results of Mee et al. show that the relative magnitude of the individual loss components of the tested cascade vary with Mach number. The results are specific to the cascade tested, and should therefore be considered representative and not universal. Boundary layer losses decreased roughly linearly with increasing Mach number due to the thinning of the boundary layers. At low subsonic Mach numbers, the boundary layer losses contributed to the majority of the total losses. As the boundary layer losses decrease, the mixing losses increased to give constant total loss at subsonic Mach numbers.

Mixing losses were calculated by subtracting the boundary layer and shock losses from the total losses. The mixing losses primarily represent the trailing edge losses. However, Mee et al. note that shock losses which occur downstream of the traversing plane will influence the mixing losses.

Shock losses begin to appear just below Mach 1.0. The outlet Mach number quoted is the average downstream value. Before this value reaches 1.0, there are local patches of supersonic flow, ending in shocks, on the aft part of the suction surface. Hence, some shock losses are generated before the downstream flow is sonic. The shock losses are calculated from the total pressure deficit in the flow outside of the blade wake. 
Near Mach 1.0, the three loss components are roughly equal in magnitude. As the Mach number is increased, the shocks become increasingly oblique. Consequently the shock losses decrease slightly.

These three components of loss are not independent. Shock waves that form at the trailing edge of one blade can influence the pressure distribution on the suction surface of the adjacent blade, thus altering the boundary layer losses. In some cases, the shock wave-boundary layer interaction can lead to separation; this will be discussed later in the chapter.

\subsection{Aerodynamic Influences on Midspan Losses}

\subsubsection{Compressibility Effects}

In transonic cascade measurements, the Mach number at the exit of the cascade is often a parameter which is easily varied in the experiment. Consequently, high-speed studies of turbine losses often cover a range of outlet Mach numbers. However, common methods for varying Mach number inherently vary the Reynolds number as well. Relatively few high-speed studies isolate the effects of Mach number from Reynolds number. Mee et al. (1992), whose results covered a broad range of Mach numbers, and were obtained at a constant Reynolds number of $10^{6}$, is one such study. Additionally, Corriveau (2005) measured losses at several transonic Mach numbers, each over a range of Reynolds numbers for both design and off-design incidence. The author of this thesis is not aware of other such studies.

At constant Reynolds number, the direct effect of Mach number on boundary layer growth is slight (Denton, 1973). Therefore, boundary layer losses are affected primarily by the influence of Mach number on the blade surface velocity distribution. As the outlet Mach number approaches 1.0, losses are also influenced by the formation of shocks in the blade passage. These shocks contribute to the losses in two ways: through direct entropy rise across the shock and through interactions between the shocks and the blade surface boundary layers. Shock-boundary layer interaction has been described by Graham \& Kost (1979), and later, in more detail, by Delery \& Marvin (1986).

Shocks can form in the flow passage at outlet Mach numbers as low as 0.6. Near the leading 
edge, depending on the inlet flow angle and leading edge geometry, regions of supersonic flow can also arise. These regions of supersonic flow often will be terminated in a shock. Turbines with high inlet Mach numbers are often designed with thin leading edges to avoid, or reduce the impact of these shocks.

Increasing the outlet Mach number to about 0.9 can result in a region of supersonic flow just downstream of the throat, which ends in an oblique shock. This oblique shock moves downstream towards the trailing edge of the blade as the Mach number approaches 1.0. Above Mach 1.0, the shock becomes increasingly oblique with Mach number, propagates downstream, and does not interact with adjacent blades. Further discussion of the trailing edge shocks follows in Section 2.5.2.

Around Mach 1.0, shocks are formed at the compression corners created as the flow closes behind the finite trailing edge. These shocks can propagate across the flow passage and interact with the boundary layer on the suction surface of adjacent blades. As the Mach number increases, the location at which the impinging shock meets the suction surface moves towards the trailing edge, becoming more oblique. The impinging shock influences the suction side boundary layer. Figure 2.4 is a schematic representation of the the boundary layer shock wave interaction.

A separation bubble can form at the foot of the incident shock. The pressure disturbance caused by the shock propagates upstream through the subsonic part of the boundary layer. As the boundary layer deflects around the separation bubble, compression waves form upstream and combine to form a separation shock. The boundary layer fluid then passes through the impinging shock. Expansion waves can be generated as the separated shear layer turns back towards the surface. If the boundary layer reattaches, the deflection that occurs to align the boundary layer with the blade surface will result in compression waves and a reattachment shock. All of these features will increase the losses generated in the boundary layer. Furthermore, the mixing losses associated with the separation bubble will convect downstream and add to the total midspan losses. Depending on the strength of the impinging shock, and the state of the boundary layer, the boundary layer might separate entirely and not reattach. This would result in a large increase in losses. 

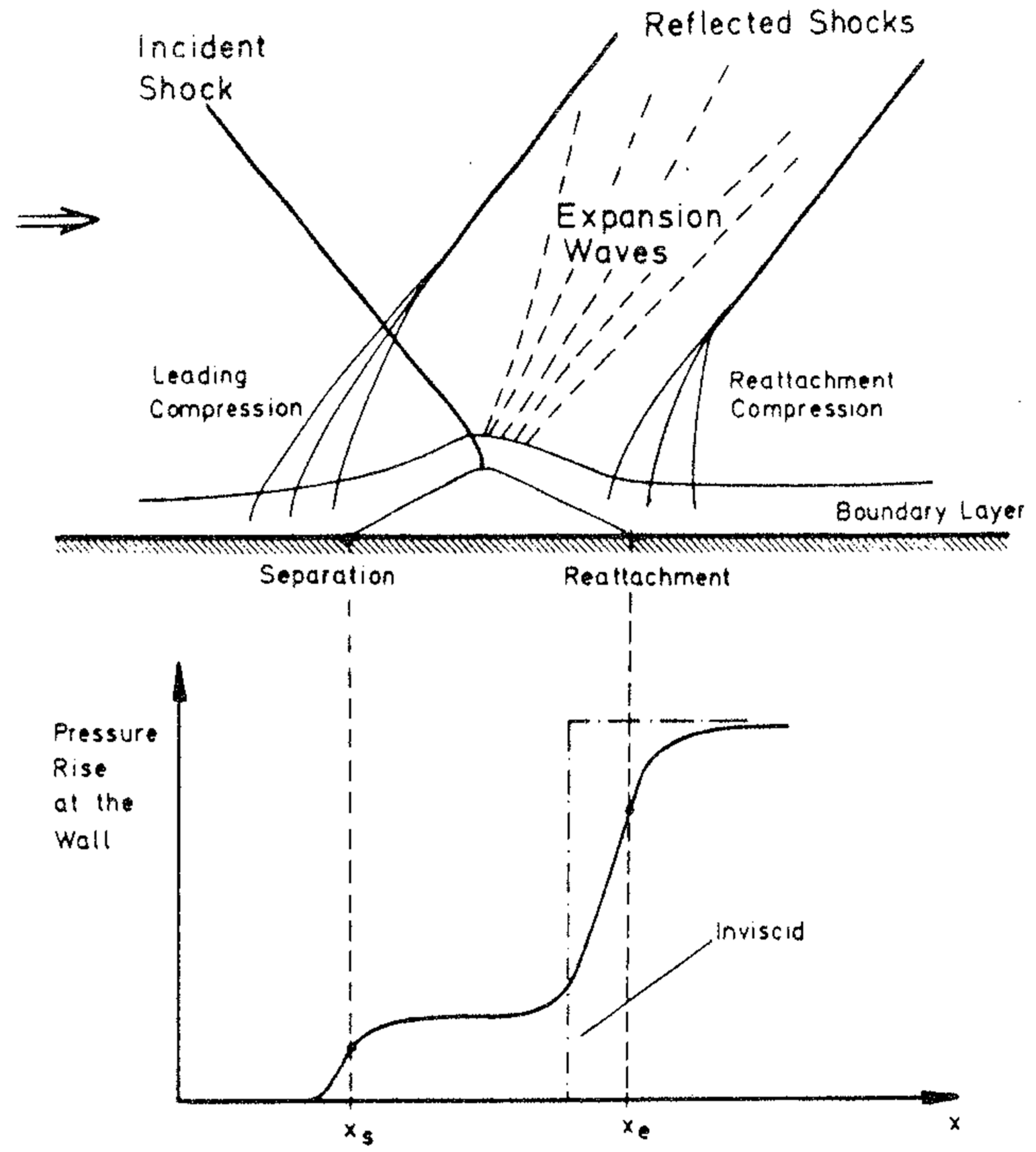

Figure 2.4: Shock Wave-Boundary Layer Interactions (Graham \& Kost, 1979) 


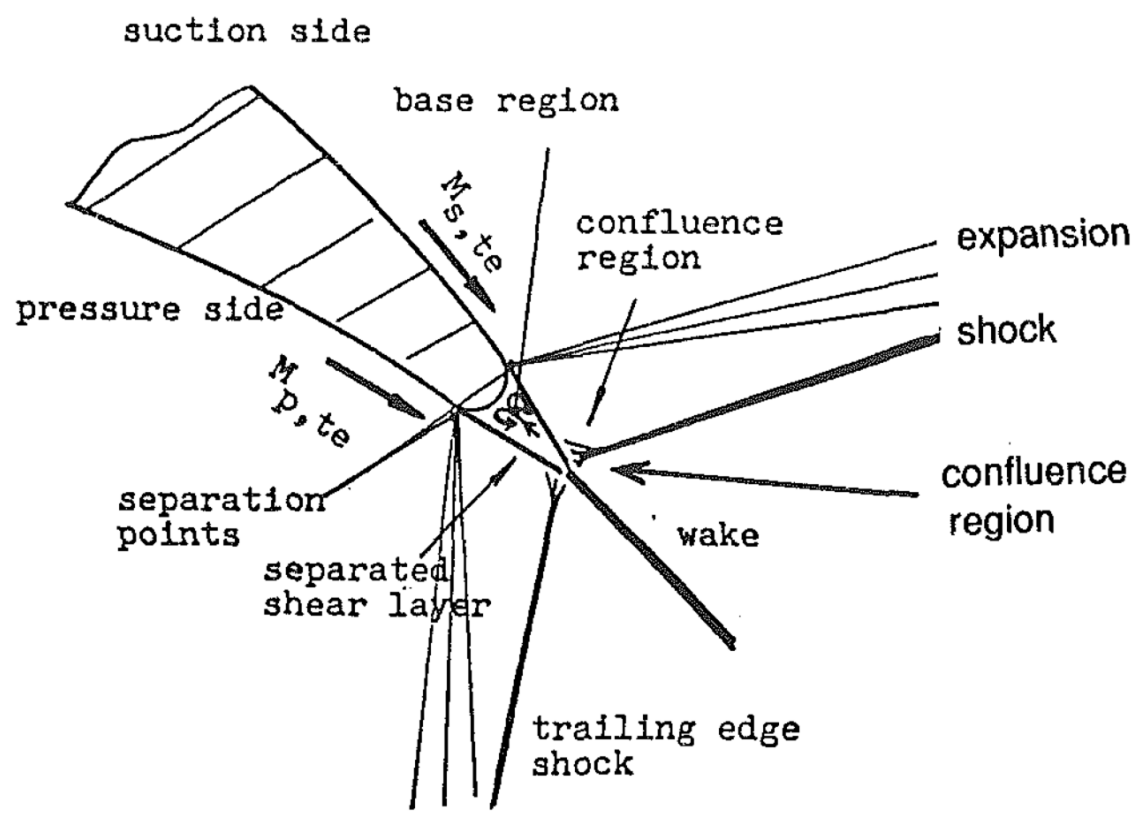

Figure 2.5: Trailing Edge Shock System (Denton \& Xu, 1990)

\subsubsection{Base Pressure}

A major contribution to the midspan losses is generated at the trailing edge of the blade. Flow in the region surrounding the trailing edge of transonic cascades is particularly complicated. However, trailing edge flows have been studied extensively (see for example: Denton $\& \mathrm{Xu}, 1990$; Mee et al., 1992; and Denton, 1993). Figure 2.5 from Denton \& Xu (1990) shows the major flow features at the trailing edge.

The blade surface boundary layers separate near the start of the trailing edge curvature, resulting in large amounts of viscous dissipation. Flow expands into the low pressure region just downstream of the trailing edge, called the base region. The pressure in the base region is nearly uniform and is called the base pressure. The change in flow direction which results from the separated flow expanding to the low base pressure gives rise to expansion waves which propagate into the free stream and can influence the adjacent blades. The separated shear layers meet in the confluence region, where they turn to a common flow direction. Compression shocks are often generated by the change in direction.

Using a control volume analysis of the flow downstream of the trailing edge, Denton (1993) 
gives the following approximate expression for the total pressure loss coefficient as a function of base pressure, $P_{b}$, and the boundary layer displacement and momentum thicknesses $\left(\delta^{*}\right.$ and $\theta)$ :

$$
Y_{t e}=\frac{\Delta P_{o}}{\frac{1}{2} \rho V_{2}^{2}}=-\frac{C_{P_{b}} t}{o}+\frac{2 \theta}{o}+\left(\frac{\delta^{*}+t}{o}\right)^{2}
$$

where the base pressure coefficient, $C_{P_{b}}$ is,

$$
C_{P_{b}}=\frac{P_{b}-P_{2}}{\frac{1}{2} \rho V_{2}^{2}}
$$

$o$ is the throat width and $t$ the blade thickness at the trailing edge. Corriveau (2005) showed that $C_{P_{b}}$ varies with Mach number and geometry. Both positive and negative values of $C_{P_{b}}$ were measured by Corriveau.

Equation 2.9 shows that the influence of base pressure on losses varies as the trailing edge blockage, $t / o$. High pressure (HP) turbines will often have much higher trailing edge blockages than low pressure (LP) turbines due to the need to cool the trailing edge of HP turbines. Consequently, low base pressure contributes more to the losses of HP turbines than of LP turbines. The second term of Equation 2.9 represents the loss contribution from the separated shear layers as they mix downstream. The losses produced in this mixing are dependent on the momentum thickness of the blade surface boundary layers. The final term accounts for the losses generated by the sudden expansion in flow area at the trailing edge, including the effective area change caused by the displacement effect of the boundary layers.

\subsubsection{Reynolds Number and Free Stream Turbulence Intensity}

The influence of Reynolds number on turbine cascade losses has been studied extensively. For example, Baines et al. (1986), Hourmouziadis (1989), Mayle (1991), and Ladwig \& Fottner (1993) have all measured the effect of Reynolds number on losses over a wide range of values.

Reynolds number is generally defined based on the outlet flow conditions as follows:

$$
R e=\frac{\rho_{2} V_{2} c}{\mu_{2}}
$$

where $c$ is the chord length of the turbine, and $V_{2}, \rho_{2}$ and $\mu_{2}$ the velocity, density and viscosity at the cascade outlet. The variation of losses with Reynolds number observed by 


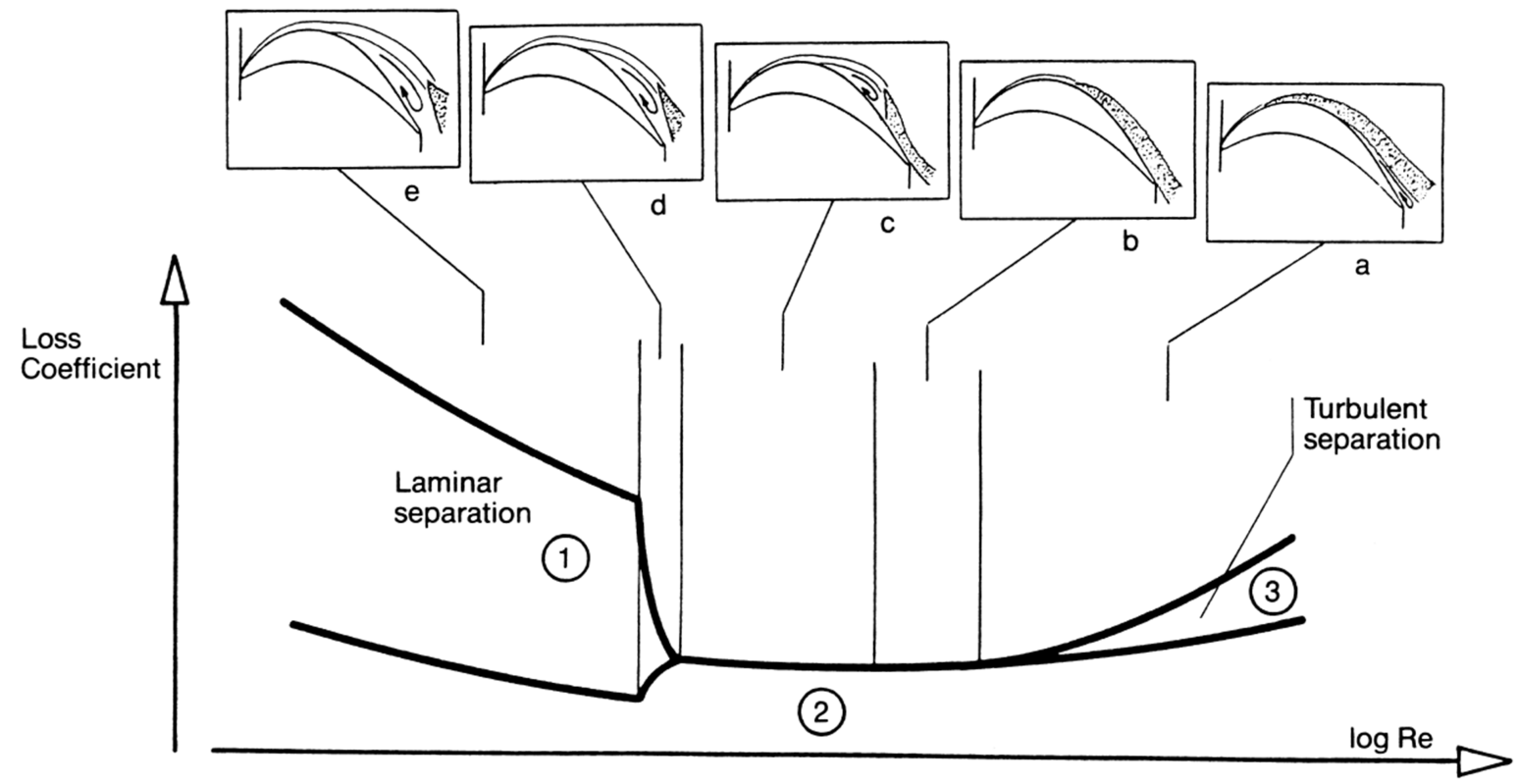

Figure 2.6: Influence of Reynolds Number on Turbine Cascade Performance Hourmouziadis, 1989)

Hourmouziadis is shown in Figure 2.6. The data of Hourmouziadis covers a Reynolds number range of $1 \times 10^{5}$ to $4 \times 10^{6}$.

The Reynolds number affects losses through its influence on the location at which transition from laminar to turbulent flow occurs on the suction side. When the outlet Reynolds number is high, the suction surface boundary layer transitions from laminar to turbulent flow near the leading edge. Any turbulent separation that may be present near the trailing edge will cause additional mixing losses. With a reduction in Reynolds number, turbulent separation may disappear and the losses will decrease. Losses will continue to decrease with decreasing Reynolds number since the transition location will move towards the trailing edge and less of the blade surface flow is turbulent. Losses reach a minimum in this regime. Further reduction of Reynolds number causes the transition location to move aft of the laminar separation point. Hence, separation will occur and the losses will increase. However, the flow will often reattach after transition to turbulence occurs in the free shear layer of the separation bubble. Continuing to reduce the Reynolds number causes the transition location, and the reattachment point, to continue to move downstream, increasing the size of the separation 


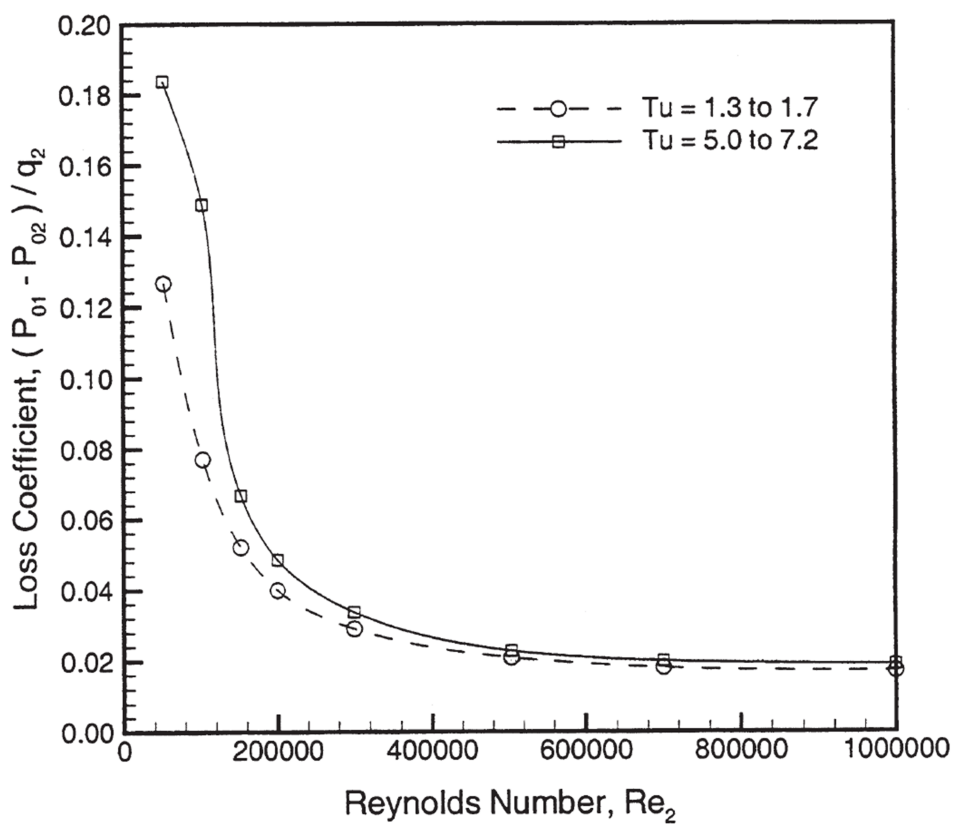

Figure 2.7: Total Pressure Loss Coefficient Variation with Reynolds Number at $M_{2}=0.59$ (Ladwig \& Fottner, 1993)

bubble and further increasing losses. At a point, the flow will not be able to reattach before the trailing edge, resulting in the highest losses.

Several authors also noted a relationship between the free stream turbulence intensity, $T u$, and the loss variation with Reynolds number. Ladwig \& Fottner (1993) measured losses at two values of turbulence intensity: $1.5 \%$ and $6.0 \%$. The free stream turbulence intensity of the Carleton high-speed wind tunnel is about 4\% (Jeffries, 2000; Jouini, 2000; Corriveau, 2005) and the free stream turbulence values in low pressure turbines can vary from $1 \%$ up to $8 \%$ (Binder et al. 1989). The data of Ladwig \& Fottner were obtained at an exit Mach number of 0.59 and spanned a range of Reynolds numbers from 50,000 to 1,000,000. Their results are shown in Figure 2.7.

Figure 2.7 shows that the losses decrease with increasing turbulence intensity at low Reynolds numbers. Increasing the turbulence intensity causes early transition in the separated shear layer, shortening the separation bubble. Ladwig \& Fottner show only a weak dependence on free stream turbulence at the higher Reynolds numbers. However, the results shown in Figure 2.7 are limited to Reynolds numbers below 1,000,000. At high Reynolds 


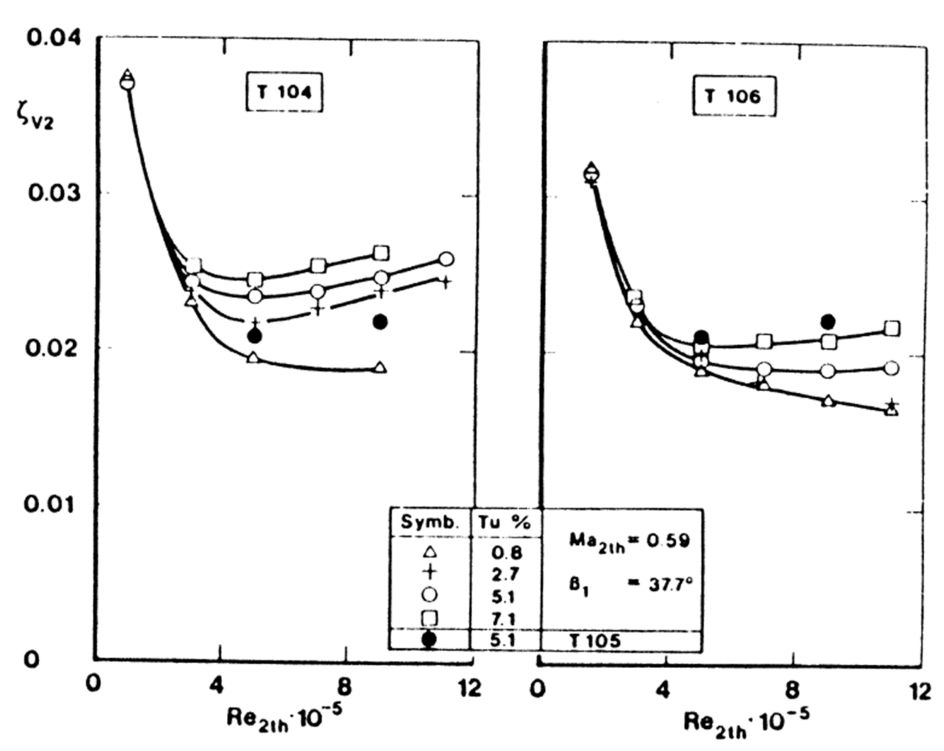

Figure 2.8: Total Pressure Loss Coefficient Variation with Reynolds Number at Different Turbulence Intensities (Hoheisel et al., 1987)

numbers, Hoheisel et al. (1987) have shown that an increase in turbulence intensity increases the losses produced in the boundary layer for the reasons explained by Hourmouziadis. These results are shown in Figure 2.8 .

\subsubsection{Surface Roughness}

Typically, for cascade testing, the turbine blades are usually hydraulically smooth. The presence of roughness affects the magnitude of losses and particulary their variation with Reynolds number. In practice, turbine blades may become rough due to corrosion, erosion, or deposition during operation. Increases in surface roughness leads to the degradation of turbine performance. Roughness of turbines removed from operating engines has been measured by Taylor (1990) and Tarada \& Suzuki (1993). Roughness tends to increase with service time, however, there was no general correlation between in-service hours and roughness height, $e$. Typical roughness heights measured ranged between 20-150 $\mu \mathrm{m}$ centreline average (CLA). Roughness is often expressed in terms of an equivalent sand-grain roughness, which corresponds to the size of uniformly distributed sand grains that yields the equivalent skin friction as the actual roughness. Using a correlation from Koch \& Smith (1976), Kind et al. (1998) 
indicate that 20-150 $\mu \mathrm{m}$ corresponds to sand-grain roughness-to-chord ratios, $e_{s} / c$, of about 0.0025 to 0.02 for for a blade with a $50 \mathrm{~mm}$ chord.

Kind et al. (1998) investigated the effects of roughness on turbine profile losses in incompressible flow. The turbines studied had span-wise roughness bands of varying lengths placed on the suction and pressure surfaces. Roughness height, spacing between roughness elements, and the location of the band in relation to the leading edge, were varied. Their findings show that roughness has little effect on the loadings of the blade. In the absence of separation, roughness also has little effect on the flow deviation at the trailing edge. Roughness on the surface thickens the boundary layers. Consequently, due to the increased blockage, the passage flow accelerates, which tends to have a greater effect on the suction surface flow due to the inherently higher velocities present there. This also leads to roughness on the suction surface having a greater effect on losses than would the equivalent amount of roughness on the pressure surface.

The effect of roughness on losses for compressible flow turbines has been studied by Jouini et al. (1998), Yuan (2004) and Zhang \& Ligrani (2004, 2006). The investigations of Yuan were closely related to those of Kind et al. (1998) for incompressible flow. Loss measurements were obtained over a range of exit Mach numbers from 0.40 to 1.13, and Reynolds numbers up to $1,000,000$ for cascades with a number of roughness configurations, including a smooth case. Like Kind et al. Yuan found little influence of roughness on the blade loadings and flow deviation. At subsonic Mach numbers, losses are sensitive to roughness height: greater roughness causes higher losses. The largest contribution to an increase in loss production is thought to be caused by roughness-induced early transition. Near Mach 1.0, losses are less sensitive to roughness due in part to the increasing contribution to losses from low base pressure. Furthermore, Yuan suggests that thicker boundary layers tend to increase base pressure, reducing trailing edge losses and partly offsetting the increase in boundary layer losses. At higher Mach numbers, the additional blockage of the roughness-thickened boundary layers causes higher acceleration, and consequently higher velocities in the passage. High velocities can lead to stronger trailing edge shocks, which in turn can strongly influence the pressure distribution of adjacent blades, potentially causing separation. Therefore, despite 


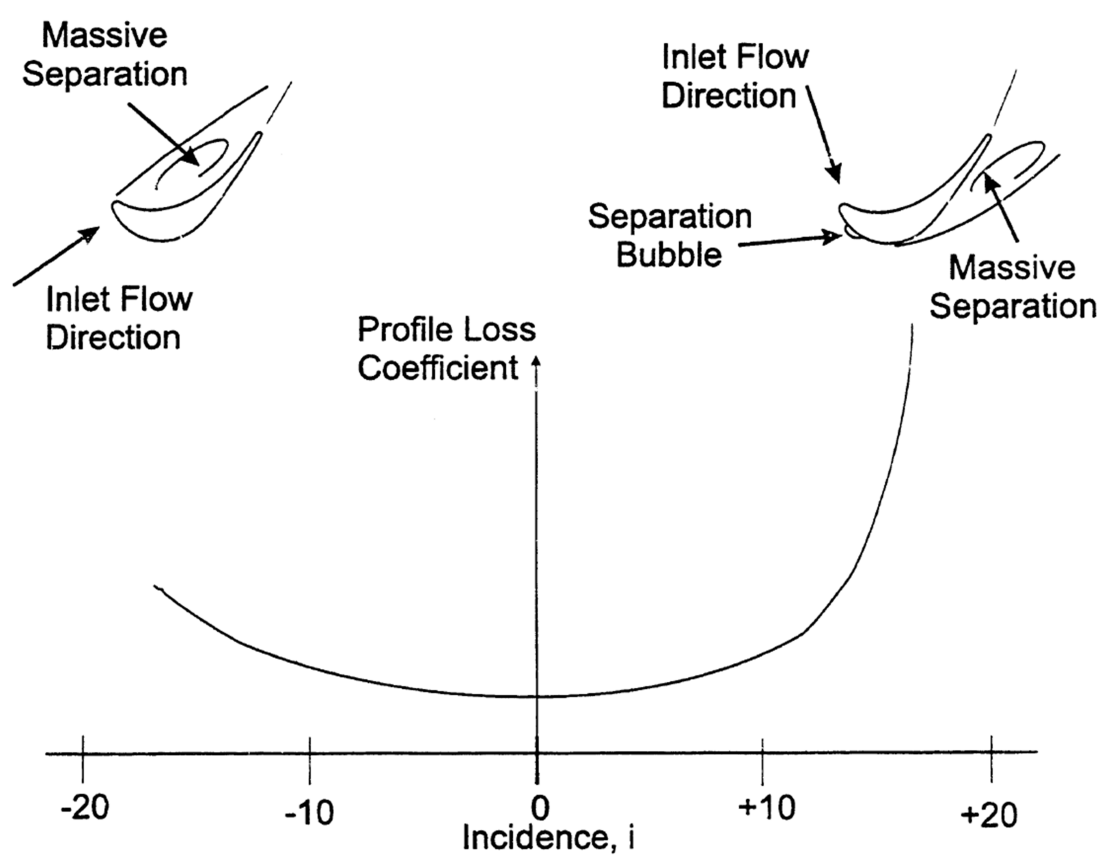

Figure 2.9: Loss Behaviour at Off-Design Incidence (Mayle, 1991)

the possible favourable influence on trailing edge losses, increased roughness generally has an adverse effect on losses above Mach 1.0.

\subsubsection{Effects of Off-Design Incidence}

It is not uncommon for gas turbine engines to operate at conditions other than those for which they were designed. Operating at off-design conditions can lead to changes in the inlet flow angle; this is especially true for LP turbines. HP turbines, which immediately follow the combustion chamber, tend not to see much variation in inlet angle. The effect that operating at off-design incidence has on losses has been a topic of much interest. Turbines are typically designed to produce the least loss while operating at the design point. This is often achieved by aligning the primary flow vector with the leading edge metal angle. Deviation from the design incidence can lead to an increase in losses. Figure 2.9 shows the typical loss behaviour at off design incidence.

Losses typically increase for both positive and negative changes in incidence. When the inlet angle is reduced (negative incidence) losses will rise gradually, and a separation bubble 
can form on the pressure side of the blade. At very large values of negative incidences, the pressure surface boundary layer can separate entirely, causing a sharp increase in losses. Similarly, at positive incidence, a separation bubble will form on the suction surface near the leading edge of the blade. The separation bubble will grow with increasing incidence and eventually the boundary layer will separate entirely. Losses tend to increase more rapidly at positive incidence and therefore the design incidence is sometimes chosen to have a slightly negative bias.

\subsection{Correlation Systems for Predicting Profile Losses of Axial Turbines}

\subsubsection{Introduction}

In meanline analysis, the need to predict turbine performance arises. Empirical correlations offer a means by which turbine designers can obtain an estimate of turbine performance early in the design process. The correlations are based on simple geometric and aerodynamic parameters that are known early in the design. These correlations offer a quick and accurate gauge of performance without the need for detailed knowledge of the blade profile.

The correlations that will be described in detail here are all based upon the widely-used system proposed by Ainley \& Mathieson (1951). A few less widely used systems will then be cited and only described very briefly.

\subsubsection{Ainley \& Mathieson Loss Correlation}

Perhaps the most widely-used loss prediction system for axial turbines is that which is due to Ainley \& Mathieson (1951) and its subsequent derivatives. The Ainley \& Mathieson method offers predictions for profile losses, secondary losses, and tip leakage losses both at design and off-design values of incidence. This section will focus solely on the design incidence

predictions of profile (boundary layer and trailing edge) losses since these are the only losses measured in the present study. In addition to the losses, Ainley \& Mathieson proposed a 
method for predicting flow deviation at the trailing edge.

The Ainley \& Mathieson system uses measured loss data from the two special cases of axial inflow nozzles, for which the inlet metal angle, $\beta_{1}=0^{\circ}$, and impulse blades, for which the inlet metal and outlet flow angles are equal $\left(\beta_{1}=\alpha_{2}\right)$, to correlate the losses with the turbine geometry and flow parameters. The data were obtained over a range of outlet flow angles, $\alpha_{2}$ and pitch-to-chord ratios, $s / c$ for both nozzles and impulse blades. The majority of the results were obtained at Reynolds numbers between $1 \times 10^{5}$ to $3 \times 10^{5}$, and Mach numbers of less than 0.6 for cascades with a maximum thickness, $t_{\max }$ equal to $20 \%$ of the chord and a trailing edge thickness, $t$, roughly equal to $2 \%$ of the blade pitch. The resulting loss coefficients are shown in Figure 2.10. The values of $Y_{P\left(\beta_{1}=0\right)}$ and $Y_{P\left(\beta_{1}=\alpha_{2}\right)}$ which correspond to the four cascades of the current study, namely E1S50, E1S90, E2S50, and E2S90 are indicated on the Figure. The cascades will be described in Section 3.6.

To estimate the performance of blades which do not belong to either special case, a weighted average is used. Profile losses are weighted based on the ratio of the inlet metal angle to the outlet flow angle $\left(\beta_{1} / \alpha_{2}\right)$. The weighted expression for profile losses prediction is,

$$
Y_{P, A M}=\left[Y_{P\left(\beta_{1}=0\right)}+\left(\frac{\beta_{1}}{\alpha_{2}}\right)^{2}\left(Y_{P\left(\beta_{1}=\alpha_{1}\right)}-Y_{P\left(\beta_{1}=0\right)}\right)\right]\left(\frac{t_{\max } / c}{0.2}\right)^{\beta_{1} / \alpha_{2}}
$$

where $Y_{P\left(\beta_{1}=0\right)}$ and $Y_{P\left(\beta_{1}=\alpha_{2}\right)}$ are the losses for nozzles and impulse blades which have the same values of $\alpha_{2}$ and $s / c$ as the blade for which the predictions are being made.

The majority of the results in Figure 2.10 were obtained for what Ainley \& Mathieson call "conventional" blades. However, they anticipated the use of the loss prediction system for blades which have different values of $t_{\max } / c, t / s$, and Reynolds number. Ainley \& Mathieson offer no correction for influence of Mach number on total pressure loss coefficient.

For cascades which have a different value of $t_{\max } / c$, the losses are scaled by $k_{t}=$ $\left(\frac{t_{\max } / c}{0.2}\right)^{\beta_{1} / \alpha_{2}}$ as indicated in Equation 2.12 .

To account for the increase in losses associated with an increase in trailing edge thickness, Ainley \& Mathieson offer the correction shown in Figure 2.11. This correction is applied as a 


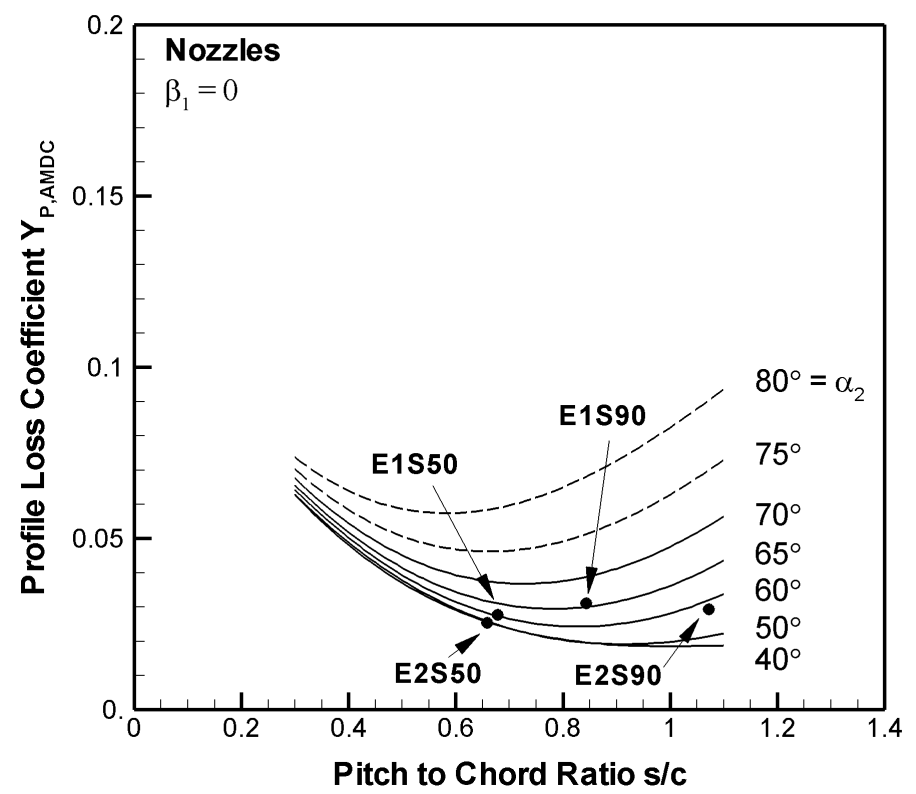

(a) Profile loss coefficient for $\beta_{1}=0^{\circ}$

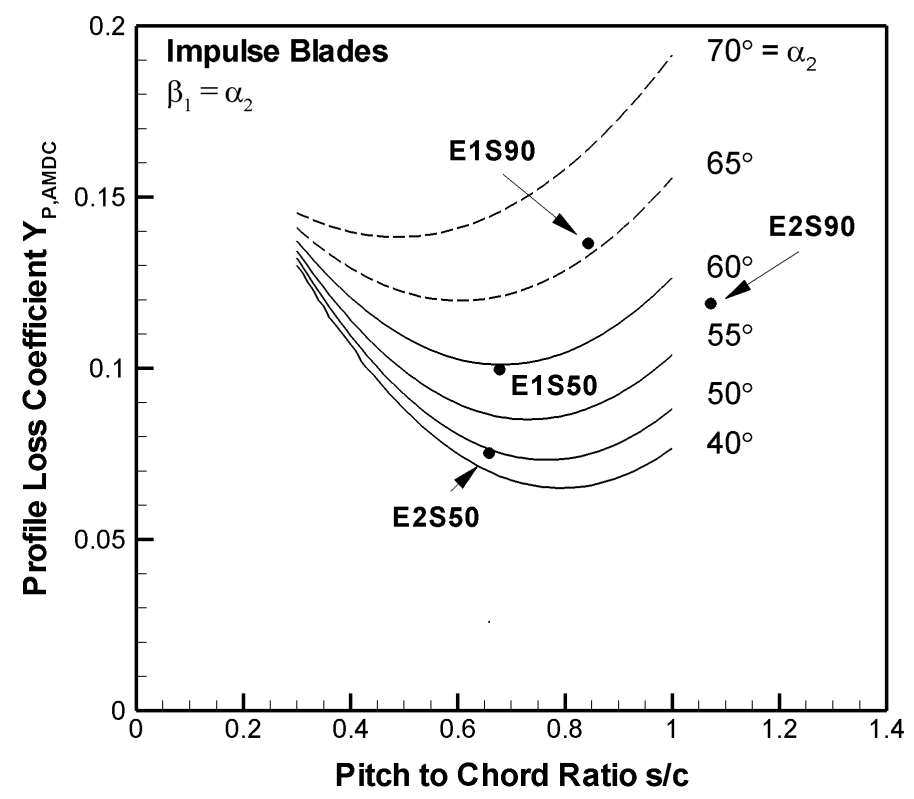

(b) Profile loss coefficient for $\beta_{1}=\alpha_{2}$

Figure 2.10: Profile Loss Coefficients at Design Incidence: $t_{\max } / c=0.2 ; R e=2 \times 10^{5}$; $M_{2}<0.6$ (Reproduced from Ainley \& Mathieson, 1951) 


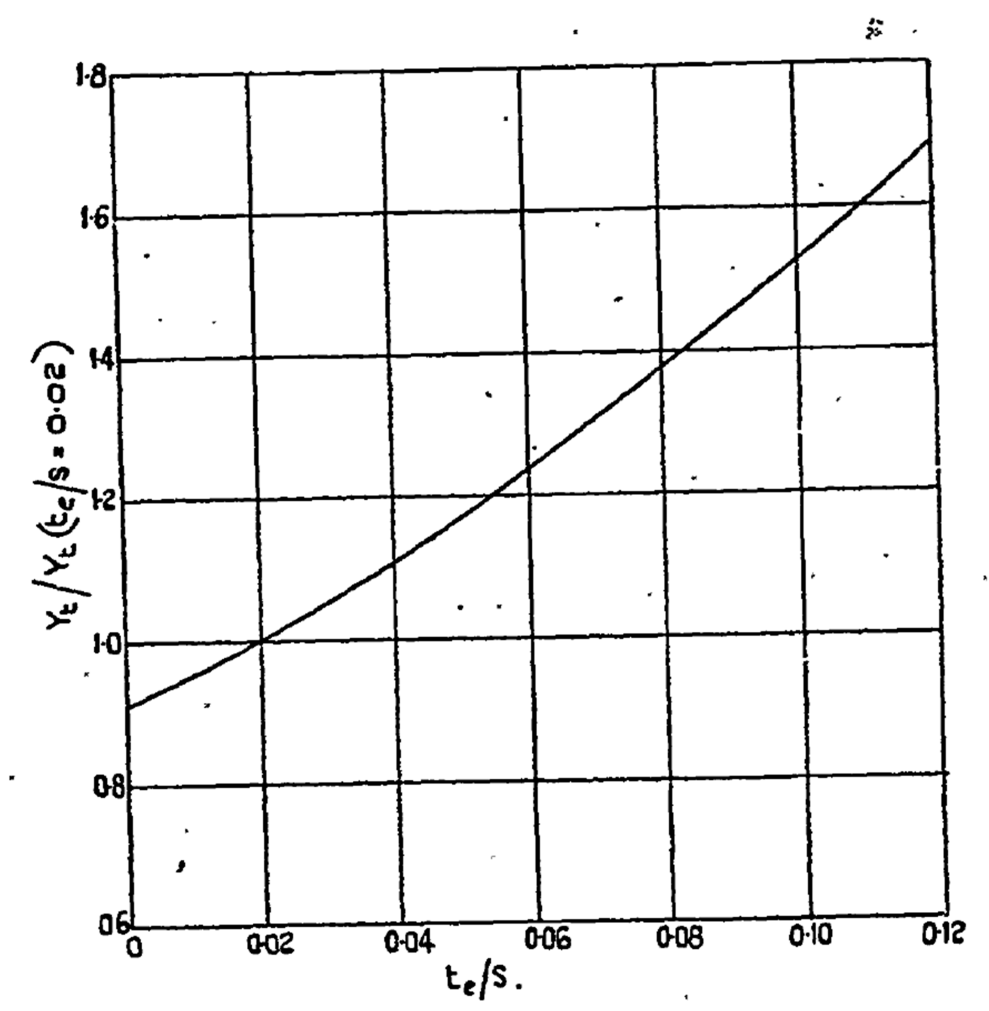

Figure 2.11: Correction Factor for Trailing Edge Thickness (Ainley \& Mathieson, 1951)

multiplier to the total pressure loss coefficient.

Finally, Ainley \& Mathieson suggest a correction for Reynolds numbers as follow:

$$
1-\eta_{\text {corrected }}=(1-\eta)\left(\frac{R e}{2 \times 10^{5}}\right)^{-1 / 5}
$$

where $\eta$ is the overall efficiency of the turbine. To estimate the efficiency of a turbine for which the outlet Reynolds number is different from $2 \times 10^{5}$, one must estimate the profile losses (Equation 2.12) as if they are independent of Reynolds number, determine the overall turbine efficiency, $\eta$, and then correct the efficiency for Reynolds number by Equation 2.13. Ainley \& Mathieson suggest that this correction is suitable for Reynolds numbers as low as $5 \times 10^{4}$, below which losses are expected to rise more rapidly.

In addition to loss prediction, Ainley \& Mathieson also offer an expression for the outlet flow angle. Below Mach 0.5, Ainley \& Mathieson suggest that the outlet flow angle is constant and is given as a function of the cascade gauge angle $\left(\cos ^{-1} o / s\right)$ and the ratio between the blade pitch and the suction surface curvature downstream of the throat $(s / e)$. For 
choked, straight-backed blades, the outlet flow angle approaches the gauge angle as Mach 1.0 is reached. This method does not take into account any influence of incidence.

\subsubsection{Modifications due to Dunham \& Came}

In the decades that followed the Ainley \& Mathieson (1951) publication, the need to update and improve the loss estimation system became apparent. This need was addressed in 1970 by Dunham \& Came. The paper compares the measured losses of 16 turbine designs with the Ainley \& Mathieson predictions. These 16 turbines were designed with the design practices current at the time of the Dunham \& Came publication. The comparisons brought to light several aspects of the loss estimations system which would benefit from modifications.

The first of these modifications concerned the Reynolds number correction. Dunham \& Came found it preferable to correct the profile and secondary loss coefficients for Reynolds number, rather than the efficiency. They did not, however, modify the correction factor itself.

Dunham \& Came also found there was much room for improvement in predicting the losses of cascades with supersonic outlet Mach numbers. Dunham \& Came introduced a compressibility correction factor $k_{c}$, which is to be applied to the profile losses if the outlet Mach number exceeds 1.0.

The resulting expression for the overall total pressure loss coefficient is,

$$
Y=\left[\left(Y_{P, A M} \times k_{c}+Y_{s}\right) f(R e)_{A M D C}+Y_{T C}\right] Y_{T E T}
$$

where $Y_{T E T}$ is the correction factor for trailing edge thickness from Ainley \& Mathieson (Figure 2.11) and not itself a loss coefficient, the Reynolds correction, $f(R e)_{A M D C}$, is,

$$
f(R e)_{A M D C}=\left(\frac{R e}{2 \times 10^{5}}\right)^{-1 / 5}
$$

and the supersonic compressibility factor, $k_{c}$, is,

$$
k_{c}=1+60\left(M_{2}-1\right)^{2}
$$

\subsubsection{Modifications due to Kacker \& Okapuu}

The loss prediction system of Ainley \& Mathieson (1951) and with the modifications of Dunham \& Came (1970) (referred to collectively as AMDC) was further improved by Kacker \& 
Okapuu (1982). Several aspects of the loss system were revisited and the final form validated against measurements from 33 turbines which, like the earlier Dunham \& Came cascades, represented the then current trends in design.

The full expression for the total pressure loss coefficient from Kacker \& Okapuu (KO) is,

$$
Y=Y_{P} \times f(R e)_{K O}+Y_{s}+Y_{T E T}^{\prime}+Y_{T C}
$$

where, $Y_{P}, Y_{s}, Y_{T E T}^{\prime}$ and $Y_{T C}$ are the loss coefficients for the profile, secondary, trailing edge, and tip clearance loss components. $f(R e)_{K O}$ is the correction factor for Reynolds number effects introduced by Kacker \& Okapuu. This can be shown to differ from the expression due to AMDC (Equation 2.14) and will be discussed further.

Inspection of Equations 2.17 and 2.14 shows that Kacker \& Okapuu modified the way in which the loss components are summed to obtain an overall total pressure loss coefficient, $Y$. In the KO system, secondary losses are no longer corrected for Reynolds number effects, and trailing edge losses have been separated entirely from the other components. Furthermore, the expressions for the loss components have been modified by Kacker \& Okapuu.

The KO expression for the profile loss coefficient is,

$$
Y_{P}=0.914\left(\frac{2}{3} Y_{P, A M D C} \times k_{p}+Y_{\text {shock }}\right) \times k_{c}
$$

There are several factors in this expression which do not appear in AMDC. First is the factor of 0.914. This corrects the AMDC nozzle and impulse blade loss coefficients to the equivalent values for zero trailing edge thickness. Additionally, a factor of $2 / 3$ is included. This represents the expected reduction in losses due to the advancements in turbine design practices that occurred between 1951 and 1982. $Y_{P, A M D C}$ is the interpolated loss coefficient from AMDC, however, with some modification; these will be addressed shortly. $k_{p}$ is a subsonic compressibility correction, which will also be discussed later. $Y_{\text {shock }}$ is a loss coefficient which accounts for the formation of shocks in the flow passage. Finally, $k_{c}$ is the previously defined supersonic drag rise multiplier from AMDC (Equation 2.16).

While generally satisfactory for predicting the profile losses for turbine blades whose inlet angle falls between impulse blades and nozzles, Kacker \& Okapuu noted that the AMDC 
expression for profile loss, $Y_{P, A M}$ (Equation 2.12, made no provision for turbine geometries with negative inlet metal angles. As a loss system which was intended for use at the meanline, where inlet angles are not generally negative, the AMDC system is adequate. However, lightly loaded turbines can sometimes be designed with negative inlet angles. Furthermore, the meanline loss systems due to AMDC or KO have subsequently been used to predict turbine performance away from the meanline in throughflow analyses of the full blade span. Near the blade tip, turbine rotors can have significantly negative values of inlet angle. This prompted Kacker \& Okapuu to modify the basic weighted expression for profile losses to,

$$
Y_{P, A M D C}=\left[Y_{P\left(\beta_{1}=0\right)}+\left|\frac{\beta_{1}}{\alpha_{2}}\right|\left(\frac{\beta_{1}}{\alpha_{2}}\right)\left(Y_{P\left(\beta_{1}=\alpha_{2}\right)}-Y_{P\left(\beta_{1}=0\right)}\right)\right]\left(\frac{t_{\max } / c}{0.2}\right)^{\frac{\beta_{1}}{\alpha_{2}}}
$$

Kacker \& Okapuu use the subscript "AMDC" to denote this profile loss coefficient, which should not be confused with the profile loss coefficient in the AMDC loss system shown in Equation 2.12 which, in this thesis, is denoted by the subscript "AM".

The AMDC loss data for nozzles and impulse blades were obtained at low subsonic Mach numbers. Consequently, the losses for turbines operating at high subsonic Mach numbers can be poorly predicted. Kacker \& Okapuu, therefore, included the subsonic compressibility correction, $k_{p}$, which is given by the following:

$$
k_{p}=1-k_{2}\left(1-k_{1}\right)
$$

where,

$$
k_{1}=1-1.25\left(M_{2}-0.2\right) \text { for } M_{2}>0.2
$$

and,

$$
k_{2}=\left(\frac{M_{1}}{M_{2}}\right)^{2}
$$

$k_{1}$ and $k_{2}$ respectively correct for influence of exit Mach number and passage acceleration on losses.

Kacker \& Okapuu also introduce a loss coefficient, $Y_{\text {shock }}$ to account for shocks that can form at the blade leading edge as a result of the local acceleration which is caused by the highly curved surfaces. 
Kacker \& Okapuu did not publish any modifications to the AMDC supersonic drag rise correction. However, they acknowledge that $k_{c}$ is independent of exit angle and therefore does not take into account airfoil limit load. The limit load is the operating point at which the tangential force is a maximum for a given inlet pressure. For turbine rotors, this has implications for the amount of work which can be extracted from the working fluid. The Mach number at which limit loading will occur is dependent on the outlet flow angle of the cascade, and so varies with cascade. Kacker \& Okapuu compare loss measurements from four transonic cascades with the AMDC predictions. They also include preliminary predictions using a new supersonic correction, the expression for which is not published. The new supersonic correction is based on the known limit load of the airfoils, which can be calculated from blade geometry.

In Equation 2.17, $f(R e)_{K O}$ is the Reynolds number correction factor proposed by Kacker \& Okapuu. $f(R e)_{K O}$ corrects for cascades with Reynolds numbers that differ from the Ainley \& Mathieson data, which primarily was for a Reynolds number of $2 \times 10^{5} \cdot f(R e)_{K O}$ is based on skin friction models and therefore has different expressions for laminar, transitional and turbulent flow; it is given by,

$$
f(R e)= \begin{cases}\left(\frac{R e_{c}}{2 \times 10^{5}}\right)^{-0.4} & \text { for } R e_{c} \leq 2 \times 10^{5} \\ 1.0 & \text { for } 2 \times 10^{5}<R e_{c}<1 \times 10^{6} \\ \left(\frac{R e_{c}}{1 \times 10^{6}}\right)^{-0.2} & \text { for } R e_{c} \geq 1 \times 10^{6}\end{cases}
$$

There is no correction in the transitional regime $\left(2 \times 10^{5}<R e_{c}<1 \times 10^{6}\right)$, where skin friction models for predicting the loss coefficient variation were not well understood. As shown in Equation 2.17, Kacker \& Okapuu correct only the profile losses for Reynolds number effects.

Similar to the profile losses, the trailing edge losses are predicted by interpolating between measured losses from the two blade types: axial inflow nozzles and impulse blades. Kacker $\&$ Okapuu proposed a relationship for the trailing edge losses of nozzles and impulse blades, shown in Figure 2.12, as a function of the trailing edge blockage, $t / o$. The losses are expressed in terms of the energy loss coefficient, $\Delta \phi_{T E T}^{2}$, defined in Section 2.3 . The relationship shown in Figure 2.12 resulted from a survey of published cascade measurements. The weighted 


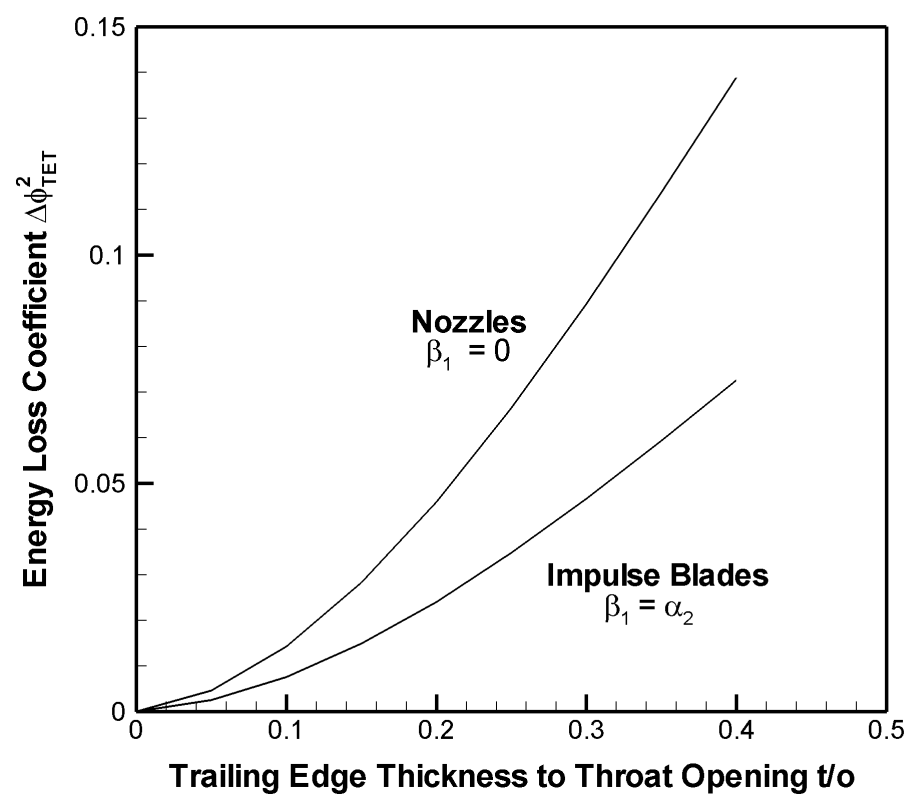

Figure 2.12: Trailing Edge Energy Loss Coefficient (Reproduced from Kacker \& Okapuu, 1982)

interpolation expression for predicting the trailing edge loss coefficient of a turbine which falls between nozzles and impulse blade geometry is,

$$
\Delta \phi_{T E T}^{2}=\Delta \phi_{T E T\left(\beta_{1}=0\right)}^{2}+\left|\frac{\beta_{1}}{\alpha_{2}}\right|\left(\frac{\beta_{1}}{\alpha_{2}}\right)\left(\Delta \phi_{T E T\left(\beta_{1}=\alpha_{2}\right)}^{2}-\Delta \phi_{T E T\left(\beta_{1}=0\right)}^{2}\right)
$$

To estimate the overall losses for a turbine, the trailing edge loss, $\Delta \phi_{T E T}^{2}$, must first be converted to a total pressure loss coefficient. The two definitions of loss coefficient vary differently with Mach number. Consequently, the conversion between total pressure and energy loss coefficients is a function of the outlet Mach number. As shown in Section 2.3, the conversion between the energy loss coefficient and the total pressure loss coefficient is,

$$
Y_{T E T}^{\prime}=\frac{\left[1-\frac{\gamma-1}{2} M_{2}^{2}\left(\frac{1}{1-\Delta \phi_{T E T}^{2}}-1\right)\right]^{-\frac{\gamma}{\gamma-1}}-1}{1-\left(1+\frac{\gamma-1}{2} M_{2}^{2}\right)^{-\frac{\gamma}{\gamma-1}}}
$$

where, $\gamma$ is the specific heat ratio of the working fluid. For incompressible flow, it can be shown that the relationship between the two definitions of loss coefficients is,

$$
Y_{T E T}^{\prime}=\frac{\Delta \phi_{T E T}^{2}}{1-\Delta \phi_{T E T}^{2}}
$$




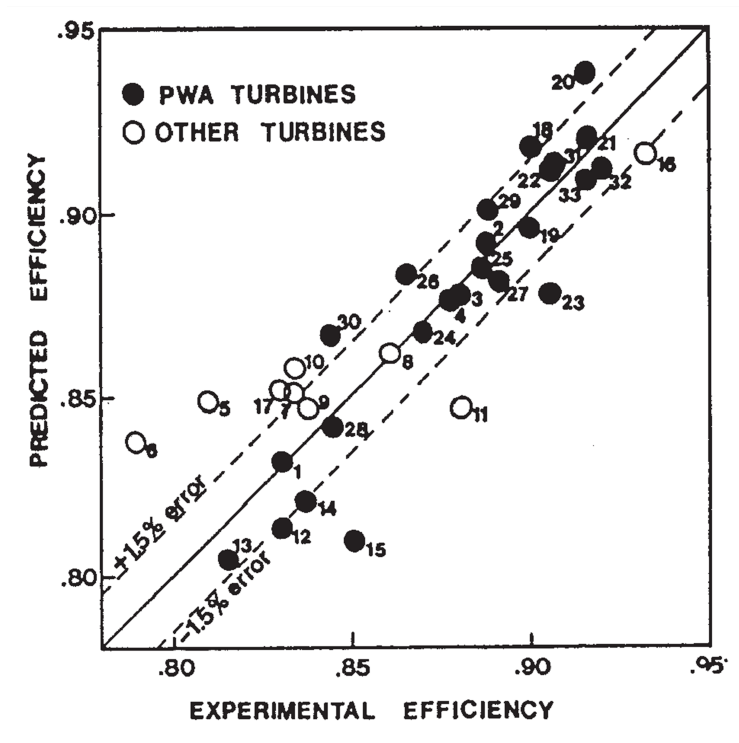

(a) Kacker \& Okapuu Prediction

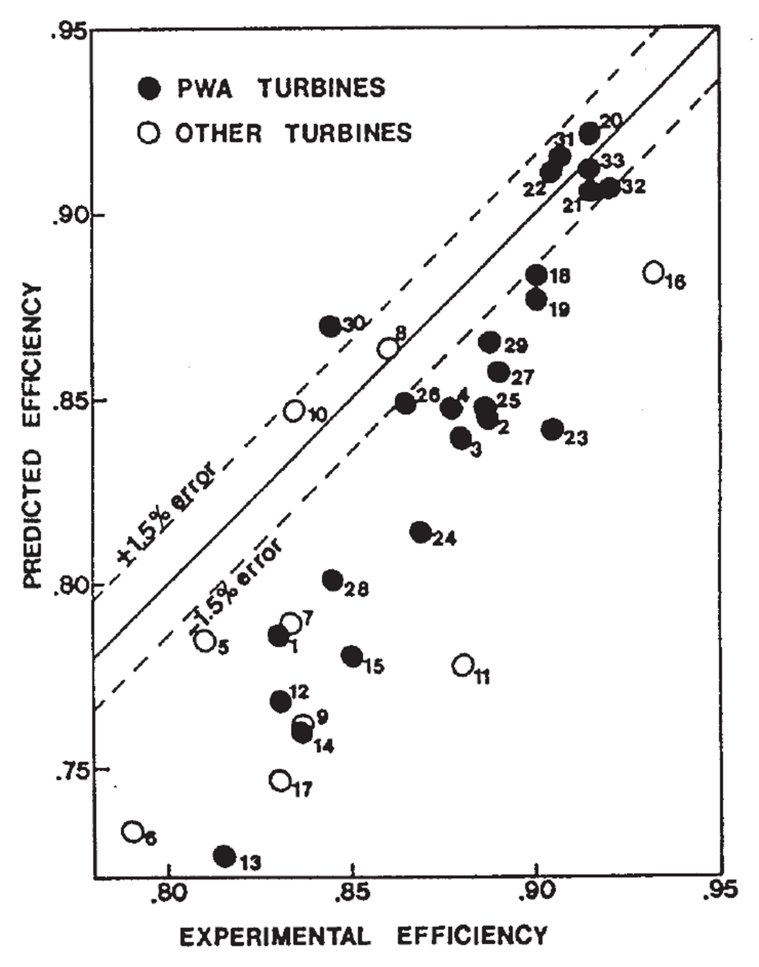

(b) Ainley \& Mathieson Prediction

Figure 2.13: Comparison of Predicted Efficiency with Measured Efficiency of 33 Turbines (Kacker \& Okapuu, 1982)

Kacker \& Okapuu validated the loss system in two ways. They first compared the efficiency predictions of 103 turbine stages which were designed using the design rules and mechanical limitations appropriate for 1950's technology. Consequently, they did not include the $2 / 3$ multiplier in Equation 2.19. The results of such predictions were compared with the well known "Smith's Chart" (see Smith, 1965). By trial and error, the subsonic compressibility correction, $k_{p}$ was adjusted to achieve reasonable agreement between the KO efficiency predictions and the Smith's Chart.

Second, design point efficiencies for 33 turbines were measured in a rotating rig. The measured efficiencies were compared both with the KO loss system as well as the AMDC system. These turbines were all of recent design at the time of the comparison and so the predicted profile losses were calculated using the factor $2 / 3$. The comparisons are shown in Figure 2.13. It is seen that the AMDC predictions significantly underestimate the efficiencies 
of the modern turbine designes, while the KO loss system results in much more accurate predictions and noticeably less scatter.

It should be noted that the supersonic correction used for the $\mathrm{KO}$ predictions differed from $k_{c}$, and is not disclosed in the paper. However, none of the 33 turbines measured had an outlet Mach number greater than 1.17 and therefore the supersonic drag rise correction had only a small effect on the efficiency.

\subsubsection{Recent Modifications to the Ainley \& Mathieson Family of Loss Cor- relations}

As was concluded by Kacker \& Okapuu, more recent researchers have also found that the loss correlations required updates to reflect design improvements. Moustapha et al. (1990) proposed an improvement to the Ainley \& Mathieson off-design incidence correlation. Based on losses from 19 cascades, Moustapha et al. introduced using the leading edge diameter as a correlating parameter. Losses are expressed in terms of the energy loss coefficient. Several years later, Benner et al. (1997) followed up the work of Moustapha et al. and further improved upon the off-design correlation. Benner et al. found that the magnitude of the discontinuity in surface curvature between the leading edge circle and the rest of the blade profile has a large effect on the blades sensitivity to changes in incidence. The leading edge wedge angle was found to give good indication of the magnitude of this discontinuity in curvature, and was therefore included as a parameter in the correlation. Both the correlation due to Moustapha et al., and that of Benner et al. is intended to be used with the design-point loss correlation of Kacker \& Okapuu (1982).

Islam \& Sjolander (1999) developed a correlation for predicting deviation to improve upon the existing deviation correlations due to Ainley \& Mathieson and Carter \& Hughes (1950). Islam \& Sjolander suggest that the greatest influence on deviation is the pressure distribution on the aft end of the blade. Blade loading is determined primarily by the amount of flow turning and the blade spacing-to-chord ratio, $s / c$. Other parameters, such as the stagger angle, $\zeta$, maximum blade thickness, and axial velocity density ratio (AVDR), were found to

also influence loading, and hence the deviation. Zhu \& Sjolander (2005) further improved the 
deviation correlation. Deviation measurements from 81 test cases, covering a wider range of the influencing parameters, led Zhu \& Sjolander to remove the influence of AVDR and include the Reynolds number as a correlating parameter.

In the same paper that proposed the improved deviation correlation, Zhu \& Sjolander (2005) also proposed several improvements to the Kacker \& Okapuu profile loss expression. Losses from 31 cascades with a total of 142 test cases were examined. The factor of $2 / 3$ used by Kacker \& Okapuu was found to be too optimistic when applied to nozzles and so was modified to 0.875 . Zhu \& Sjolander also showed that losses were over predicted for cascades which had values of $t_{\max } / c$ greater than 0.2 . Finally, Zhu \& Sjolander proposed a modification to the Reynolds number correction for low Reynolds numbers. Subsequently, Hall (2012) has suggested a preliminary correction for Reynolds number in the transitional range between $2 \times 10^{5}$ and $1 \times 10^{6}$.

\subsubsection{Alternatives to the Ainley \& Mathieson Family of Loss Correlations}

Although the Ainley \& Mathieson family of loss correlations are generally the most commonly used, several alternatives do exist. Horlock (1960), Stewart (1961), Traupel (1966), and Craig \& Cox (1971) have all proposed loss systems. Denton (1973) gives a short summary of several of these correlations and others, and compares their accuracy against measured and calculated losses. Denton suggests that, while the correlations are reasonably accurate at predicting losses for the cascades upon which the correlations are based, they fail to adequately predict losses of a range of cascades from different sources. However, of those examined, that due to Baljé \& Binsley (1968) proved the most accurate. It is important to note that this comparison was made before the modifications to the Ainley \& Mathieson correlation, as described above, were made. 


\section{Chapter 3}

\section{Experimental Apparatus}

\subsection{Introduction}

This chapter describes the experimental set-up, measurement equipment, and the turbine cascades used in this study. The experimental data were obtained with the Pratt \& Whitney Canada (PWC) high-speed wind tunnel (HSWT) at Carleton University in Ottawa, Canada. The chapter opens with a description of the HSWT facility, including details of the wind tunnel test section, as well as all supporting equipment. A description of the data collection instrumentation, such as pressure transducers and pressure probes, will follow. The chapter concludes with an overview of the turbine cascades used for this investigation.

\subsection{Carleton University's PWC High-Speed Wind Tunnel}

\subsubsection{Wind Tunnel Overview}

The Pratt \& Whitney Canada (PWC) high-speed wind tunnel (HSWT), shown in Figure 3.1. is an intermittent blow-down wind tunnel capable of sustaining stable transonic flow for as long as 60 seconds. The HSWT was originally designed and constructed in the early 1970's under the supervision of Prof. J.H.T Wade at McMaster University in Hamilton, Canada (Jeffries \& Sjolander, 1995). In the 1990's, the equipment was acquired by Carleton University's Mechanical and Aerospace Engineering department. It was commissioned and its capabilities were extended by Jeffries (1994). 


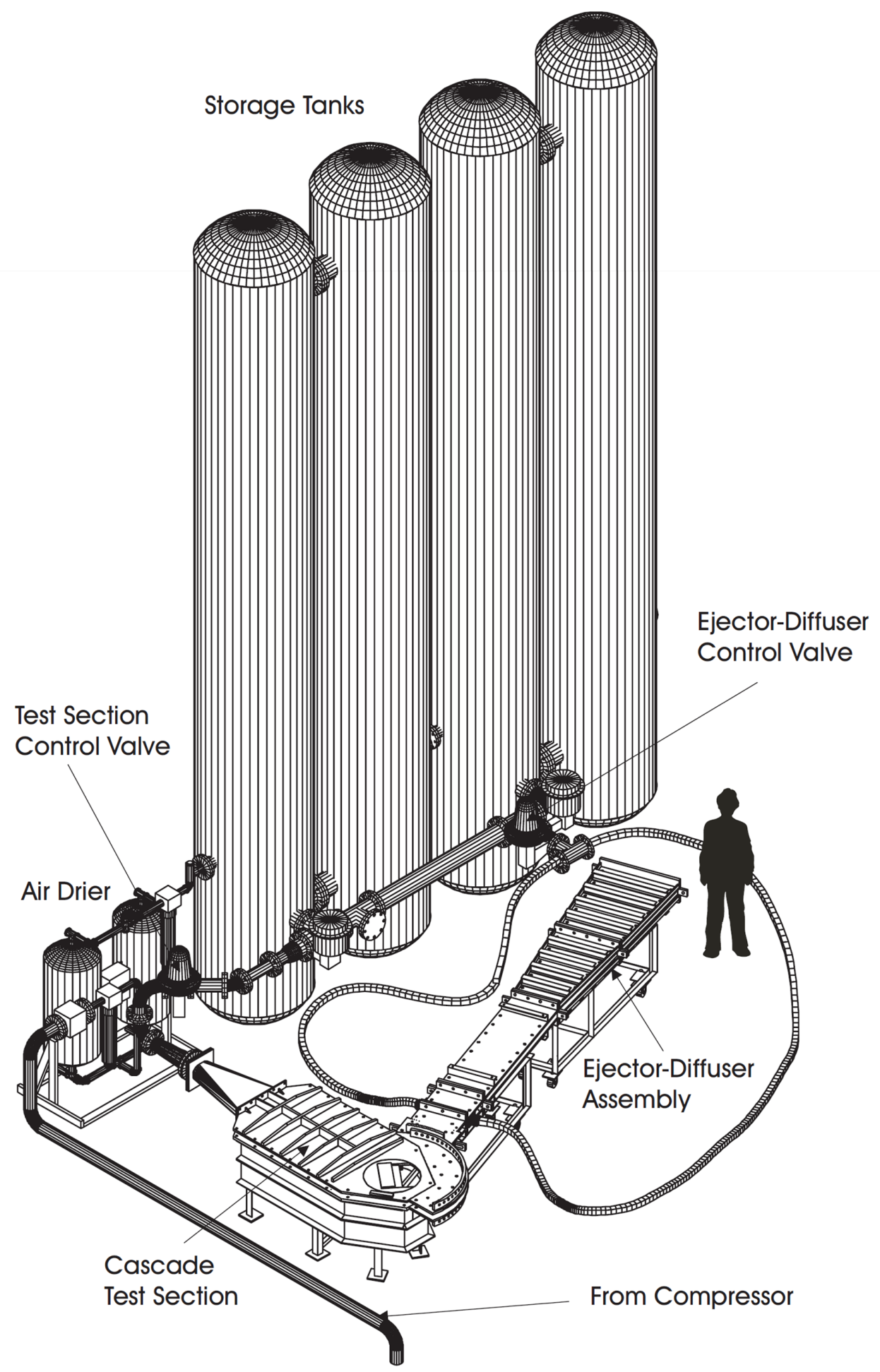

Figure 3.1: Pratt \& Whitney Canada High-Speed Wind Tunnel (Jeffries \& Sjolander, 1995) 
Compressed air is supplied to the wind tunnel at 8 atmospheres from a two-stage reciprocating compressor located several floors below the main laboratory. Before reaching the wind tunnel, the compressed air passes through a series of coolers and dryers, lowering the dew point to about $-45^{\circ} \mathrm{C}$. The air is then stored in four large pressure vessels with a combined volume of about $26 \mathrm{~m}^{3}$. Air is discharged intermittently, passes through a wire mesh that generates a known amount of turbulence, and is then directed through the test section. The measurement of the turbulence generated will be discussed further. Following the test section, the air passes through the ejector-diffusor assembly and discharges into the laboratory at atmospheric pressure. Blowing pressure is controlled with an electro-pneumatically actuated 4 inch ball valve located upstream of the turbulence grid. Run times vary with the throat area of the cascade installed and typically are of the order of 30-60 seconds.

The following sections will describe in detail the components of the HSWT.

\subsubsection{Supply Air Delivery System}

The compressor, pictured in Figure 3.2, is a Boom-Wade model VC500 two-stage intercooled compressor, driven by a $100 \mathrm{HP}$ electric motor. The compressor supplies air at about 8 atmospheres (approximately $115 \mathrm{psi}$ ) at a volumetric flow rate of about $14 \mathrm{~m}^{3} / \mathrm{min}$ (roughly $500 \mathrm{scfm}$ ). This corresponds to a mass flow rate of about $17 \mathrm{~kg} / \mathrm{min}$. At these rates, the storage tanks take about 15-20 minutes to fill to a pressure of about $690 \mathrm{kPa}(100 \mathrm{psi}(\mathrm{g}))$ from near atmospheric. As a safety measure, when the tank pressure rises above 100 psi(g), the air exiting the compressor is discharged into the room that houses the compressor. Once the pressure in the storage tanks drops below $80 \mathrm{psi}(\mathrm{g})$, tank pressurization resumes. A check valve prevents flow reversal.

The air exits the second stage of the compressor at temperatures in excess of $100^{\circ} \mathrm{C}$. Therefore, the air passes through a reverse flow aftercooler, which reduces the temperature to just several degrees above the temperature of the cooling water. Following the cooling, the air passes through a water separator which removes some moisture from the air. However, the majority of the drying occurs in dedicated dryers. The drying system, shown in Figure 3.1, is a Comp-Air Kellogg model CDH520 regenerative desiccant dryer. The air drying is alternated 


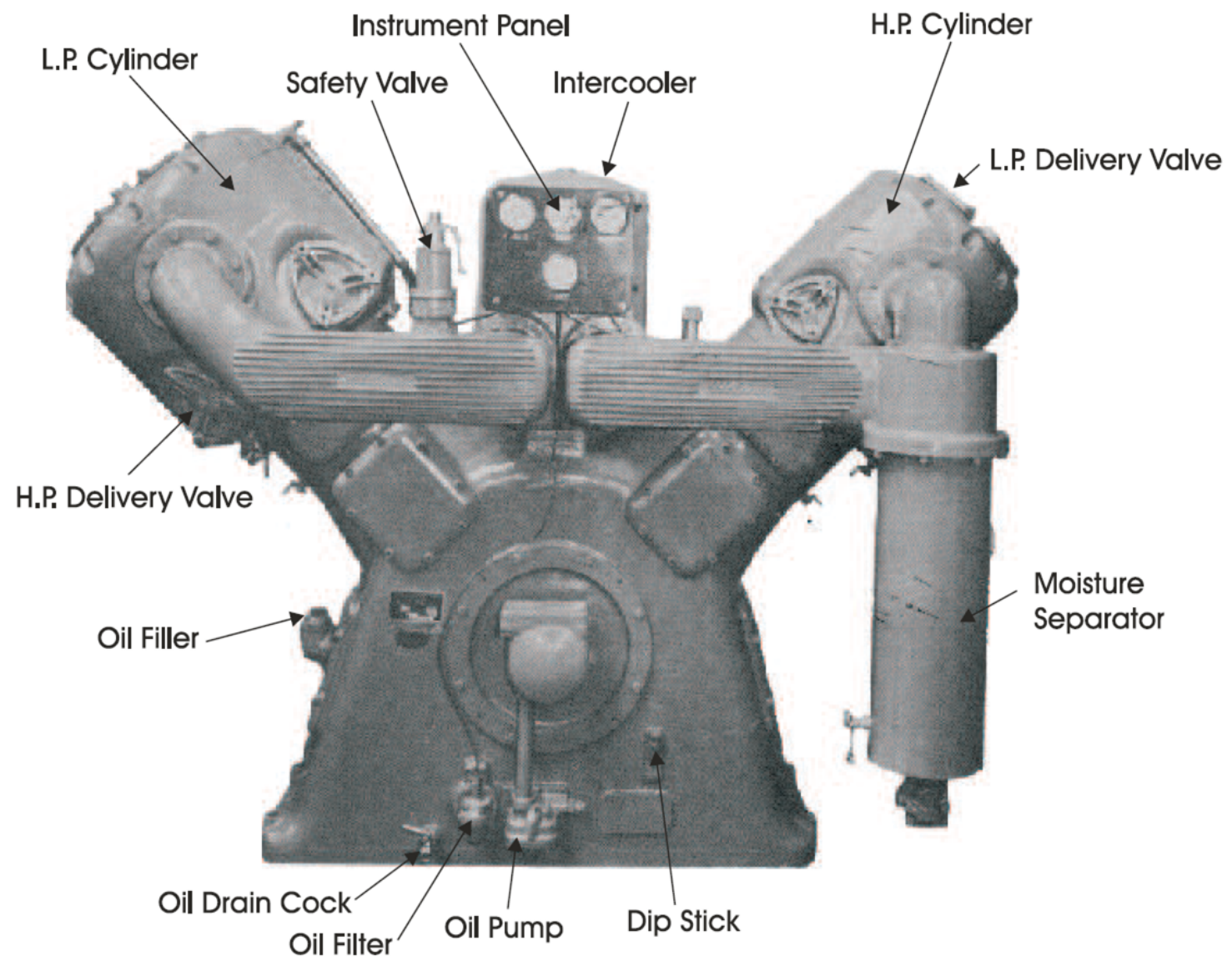

Figure 3.2: Broom-Wade VC500 Compressor (Corriveau, 2005) 
between the two tanks shown in Figure 3.1. while one tank dries the air through adsorption, the desiccant material in the other is regenerated. Air leaving the dryers has a dew point of about $-45^{\circ} \mathrm{C}$. As the tanks are discharged, the temperature of the air inside the tanks, and hence the total temperature of the air in the test section, decreases. Furthermore, the air inside the test section is highly accelerated. Consequently, the static temperature of the air inside the test section can drop well below the freezing point of water. In the absence of the dryers, condensation and icing can occur, which would damage the equipment. Furthermore, and perhaps more importantly, moisture in the air can distort the data through the formation of condensation shocks and other effects. The compressed, cooled, and dried air is stored in four large pressure vessels which have a total volume of $26 \mathrm{~m}^{3}$.

\subsubsection{Control and Shutoff Valves}

The HSWT has two flow paths: the main test section flow path, and the auxiliary flow path. The auxiliary flow path has been used in the past to supply air to the ejector-diffusor system. However, in the current study, the auxiliary system was used solely to supply air to the probe calibration rig. The probe calibration rig will be described in Section 3.5. The blowing pressure of both the main test section and the auxiliary system is independently controlled with dedicated valves. For safety, a shut-off valve, located upstream of each of the control valves, is closed when the wind tunnel is not in operation. The details of the valves, actuators, and positioners are summarized in Table 3.1 .

\subsubsection{Test Section and Ejector-Diffusor Assembly}

The main test section, shown in Figure 3.3, houses the test cascade and instrumentation. Air is supplied at the inlet to the test section where a turbulence generating grid is located. Using hot-wire anemometry, Corriveau (2005) measured the turbulence intensity and length scale at a location about $300 \mathrm{~mm}$ upstream of the cascade. The turbulence intensity was found to be $4 \%$ with an integral length scale of $15 \mathrm{~mm}$. These values were shown to be roughly constant over the range of operating conditions typically seen. A reference total pressure and total temperature are measured downstream of the turbulence grid, as shown in Figure 3.3 . 
Table 3.1: Valve Summary

\begin{tabular}{cllll}
\hline & Function & Actuator & Valve Details & Positioner \\
\hline \hline \multirow{2}{*}{$\begin{array}{c}\text { Main Test } \\
\text { Section }\end{array}$} & Shut-Off & N.J. ${ }^{1}$ QP3C-M & $\begin{array}{l}\text { 6" N.J. 815L } \\
\text { Butterfly }\end{array}$ & - \\
\cline { 2 - 5 } & Control & N.J. QP2C-M & 4" N.J. 7150 Ball & $\begin{array}{l}\text { NE724 0-20 mA } \\
\text { Electro-Pneumatic }\end{array}$ \\
\hline $\begin{array}{c}\text { Auxiliary } \\
\text { System }\end{array}$ & Shut-Off & N.J. QP3C-M & $\begin{array}{l}\text { 6" N.J. 815L } \\
\text { Butterfly }\end{array}$ & - \\
\cline { 2 - 5 } & Control & N.J. QP1C-M & $\begin{array}{l}\text { 2" N.J. R21 } \\
\text { Segmented-Ball }\end{array}$ & $\begin{array}{l}\text { NE724 0-20 mA } \\
\text { Electro-Pneumatic }\end{array}$ \\
\hline
\end{tabular}

${ }^{1}$ Neles-Jamesbury

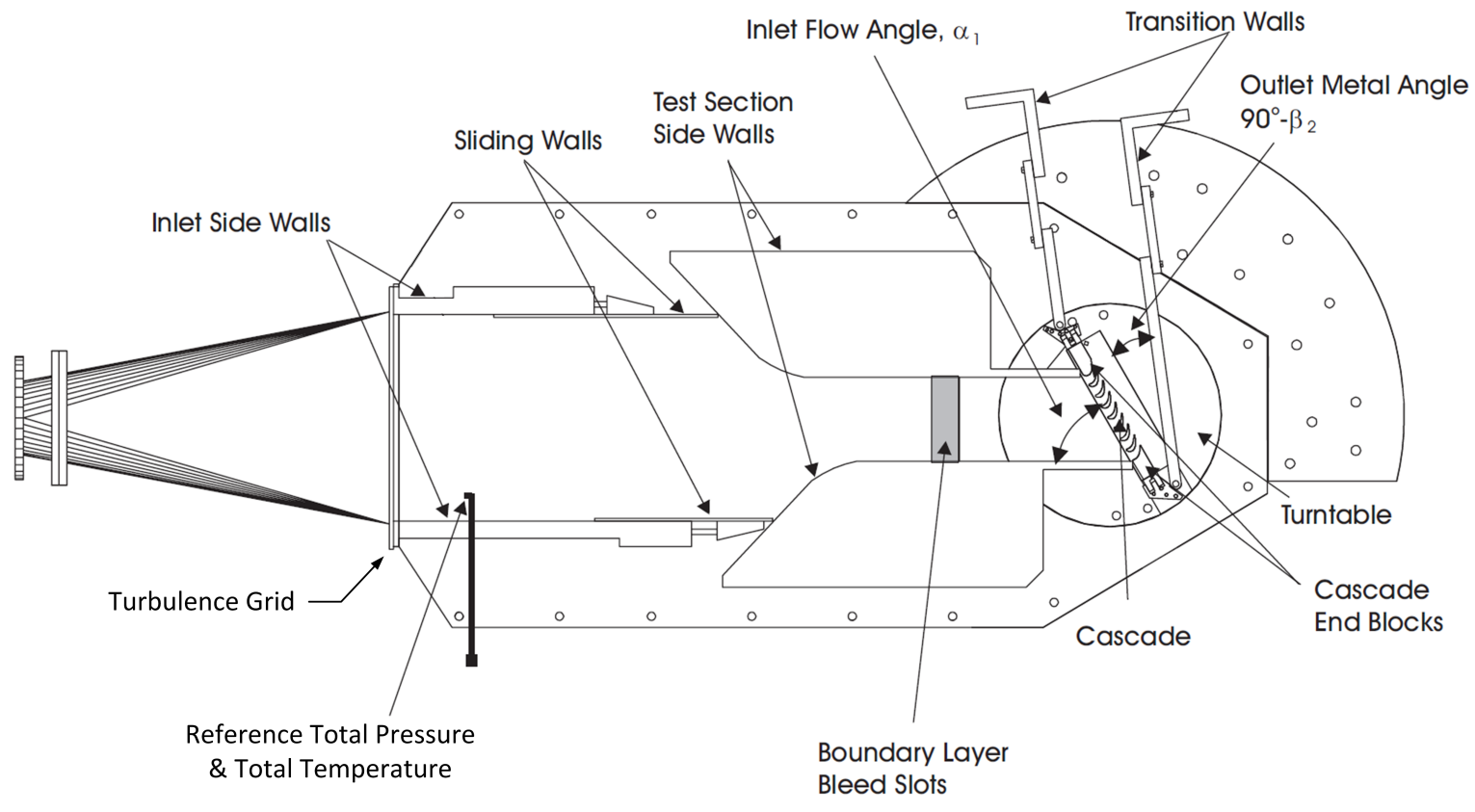

Figure 3.3: Test Section (Corriveau, 2005) 
Adjustable side walls direct the flow to the inlet of the cascade where, due to the contraction in flow area, the flow accelerates to Mach numbers typically between 0.3 and 0.5 . The side walls can be adjusted both forward and backward, and side to side to allow for cascades of varying width and inlet metal angles to be installed into the test section. Furthermore, at different incidences the walls are adjusted to account for the change in inlet flow area. The seal between the side walls and the cascade endblocks is kept tight with a spring. Upstream of the adjustable side walls, pneumatic pistons are used to maintain a tight seal between the sliding walls and the side walls. The height of the test section is $61 \mathrm{~mm}$.

The cascade is mounted on a turntable which allows the inlet flow angle to be varied over a wide range. In the current investigation, the inlet flow angle was varied between $-30^{\circ}$ and $30^{\circ}$. The turntable is equipped with a worm gear and protractor with which the inlet angle can be set with an accuracy of $\pm 0.5^{\circ}$. A clear Lexan window, instrumented with static taps upstream and downstream of the cascade, seals the top of the cascade. Downstream of the cascade, the transition walls, which can be adjusted to match the outlet metal of the cascade, attach to the ejector-diffusor assembly.

The flow exits the wind tunnel through the ejector-diffusor assembly shown in Figure 3.4 . Use of the ejector-diffusor system for independently varying the Mach and Reynolds numbers is described by Corriveau (2005). Two supersonic jets are introduced parallel to the outlet flow, which has a lower velocity than the jets. Mixing of the jets with the core test section flow entrains fluid from the core, thus lowing the static pressure downstream of the cascade. In the current study, the ejector portion was not used. For the measurements of cascade E2S90 the ejector and mixing tube were removed to allow a wider range of inlet angles to be set without interference between the diffusor and air storage tanks. The diffusor, which doubles in width over its length, provides some static pressure recovery, discharging to the laboratory at atmospheric pressure. For a given blowing pressure, the use of the diffusor lowers the static pressure downstream of the cascade, thus increasing the outlet Mach number. 


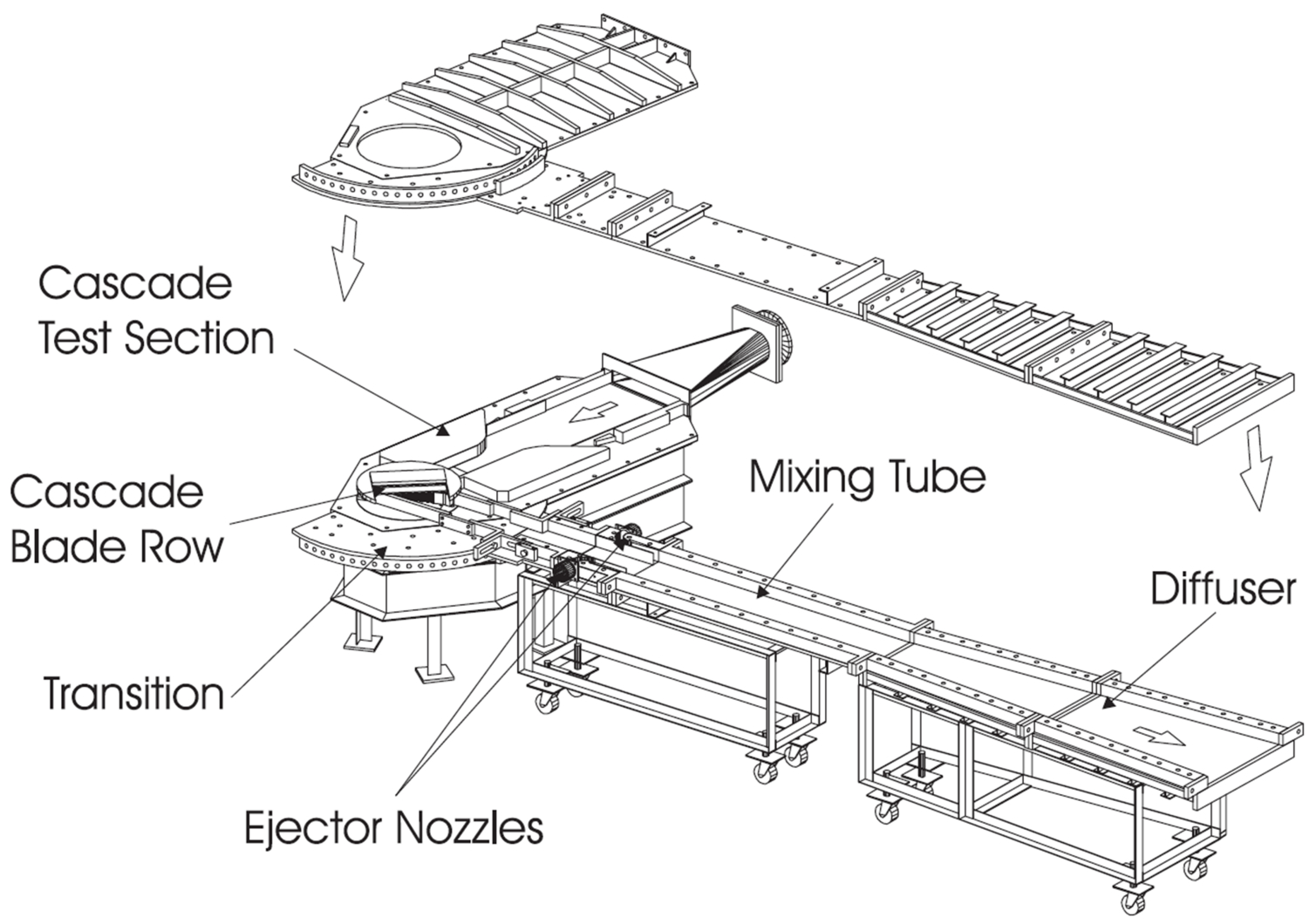

Figure 3.4: Ejector-Diffusor Assembly (Jeffries \& Sjolander, 1995) 


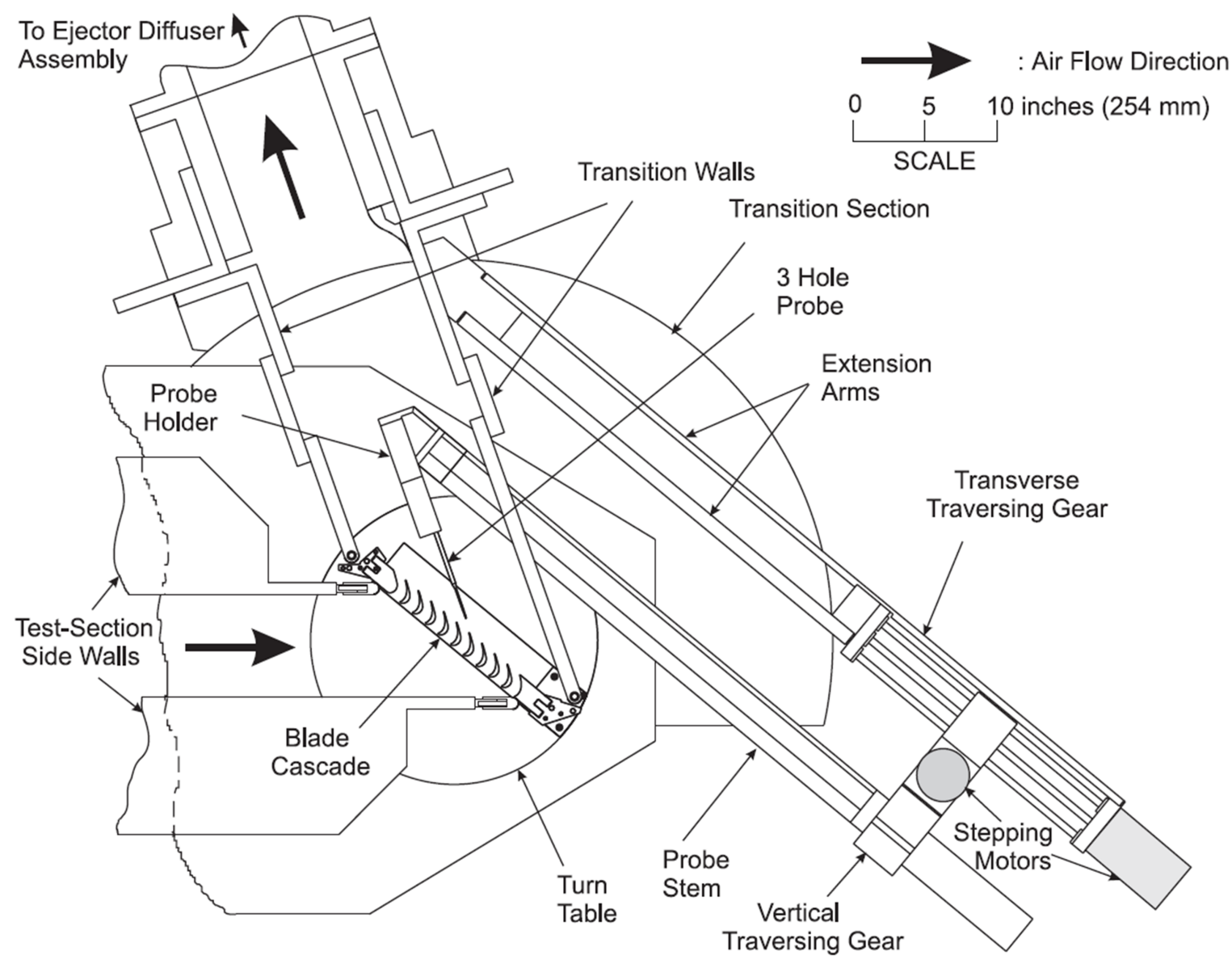

Figure 3.5: Test Section and Probe Traverse Mechanism (Jouini et al., 2002) 


\subsubsection{Probe Traverse Mechanism}

To obtain pitchwise measurements, the traverse mechanism shown in Figure 3.5 was used. The traverse gear is attached to the transition walls at a fixed angle specific to each cascade. The probe stem, on the end of which the probe holder is mounted, runs parallel to the traverse gear. The point at which the probe stem enters the test section is sealed using two Teflon blocks, which are also specific to each cascade. The angle is chosen such that as the probe traverses the cascade, it remains at a constant axial distance downstream of the trailing edge (i.e. it moves in the pitchwise direction).

Movement of the probe stem is achieved through the use of a Compu-Motor stepper motor. The motor turns a threaded rod, to which the probe stem is coupled. The stepper motor has a resolution of 200 steps per revolution, and the threaded rod has a thread pitch of 1.96 $\mathrm{mm}$. Therefore, the probe travels $1.96 \mathrm{~mm}$ for every 200 steps of the motor. Motor control is integrated into the data acquisition and control software, which will be described in Section 4.2. Although the backlash in the gear is thought to be small, the probe location is verified frequently with a ruler mounted to the traverse gear. Accuracy in setting the probe location manually is about 0.016 inches (about $0.4 \mathrm{~mm}$ ). Furthermore, to eliminate backlash error, it is suggested that the starting probe location always be approached from the same direction.

The traverse mechanism also has the capability to move in the spanwise direction. However, since the current investigation is one of profile losses, and not secondary losses, this feature was not used.

Inlet boundary layer measurements, which will be discussed in Chapter 4, were obtained on the centerline upstream of the cascade. For these, a second smaller traverse gear was mounted to the top of the test section about one axial chord length upstream of the leading edge of the cascade. The stepper motor described above is also used to drive this traverse gear. The threaded rod for this traverse gear has a pitch of $1.56 \mathrm{~mm}$. Therefore, each step of the motor displaces the boundary layer probe by about $0.008 \mathrm{~mm}$. 
Table 3.2: Druck Transducer Summary

\begin{tabular}{|c|c|c|c|c|c|}
\hline $\mathbf{N}^{\circ}$ & Range & Serial $\mathbf{N}^{\circ}$ & Single Port Probe & Three-Hole Probe & Misc. \\
\hline $\mathrm{T} 1$ & $50 \mathrm{psi}(\mathrm{a})^{1}$ & 76738 & $P_{a t m}$ & $P_{\text {atm }}$ & $P_{\text {atm }}$ \\
\hline $\mathrm{T} 2$ & 100 psi(a) & 76741 & $P_{o, r e f}$ & $P_{o, r e f}$ & $P_{o, r e f}$ \\
\hline $\mathrm{T} 3$ & $100 \mathrm{psi}(\mathrm{d})^{2}$ & 134061 & $P_{1}$ & $P_{1}$ & Tap 1 \\
\hline $\mathrm{T} 4$ & 100 psi(a) & 76740 & $P_{2}$ & $P_{2}$ & $P_{2}$ \\
\hline $\mathrm{T} 5$ & $50 \mathrm{psi}(\mathrm{d})$ & 114370 & Port Pressure & Port 1 & Tap 2 \\
\hline $\mathrm{T} 6$ & $50 \mathrm{psi}(\mathrm{d})$ & $\mathrm{B} 6228$ & - & Port 2 & Tap 3 \\
\hline $\mathrm{T} 7$ & $50 \mathrm{psi}(\mathrm{d})$ & $82842 / 834$ & - & Port 3 & Tap 4 \\
\hline $\mathrm{T} 8$ & $75 \mathrm{psi}(\mathrm{d})$ & B3116 & - & - & Tap 5 \\
\hline $\mathrm{T} 9$ & $100 \mathrm{psi}(\mathrm{d})$ & 134060 & - & - & Tap 6 \\
\hline $\mathrm{T} 10$ & $100 \mathrm{psi}(\mathrm{d})$ & B6202 & - & - & Tap 7 \\
\hline T11 & $100 \mathrm{psi}(\mathrm{d})$ & B6992 & - & - & Tap 8 \\
\hline
\end{tabular}

\subsection{Pressure and Temperature Sensors}

Measurement of the pressures within the test section are obtained using 11 Druck pressure transducers. The operating range and serial number of each transducer are shown in Table 3.2 along with the normal configuration for data collection. Calibration curves for the transducers, shown in Figure 3.6, were obtained using the Druck DPI-605 (300 psi) high-precision digital pressure calibrator. Voltages were measured at several known pressures between about -10 $\operatorname{psi}(\mathrm{g})$ and $30 \mathrm{psi}(\mathrm{g})$.

Temperature is measured with a K-type thermocouple. The thermocouple sits in a region of low velocity upstream of the cascade. Therefore, the temperature measured is taken to be the total temperature. Because no work is done by, or imparted to the air as it passes through the test section, and any heat transfer between the air and the wind tunnel walls is assumed to be negligible, the total temperature does not change from the inlet to outlet. However, due to the expansion of the air in the storage tanks, the total temperature drops over the course of a run. Therefore, the temperature and pressures are sampled concurrently.

The pressure in the four storage tanks is measured with an Omega PX613 (150 psi(g)) 


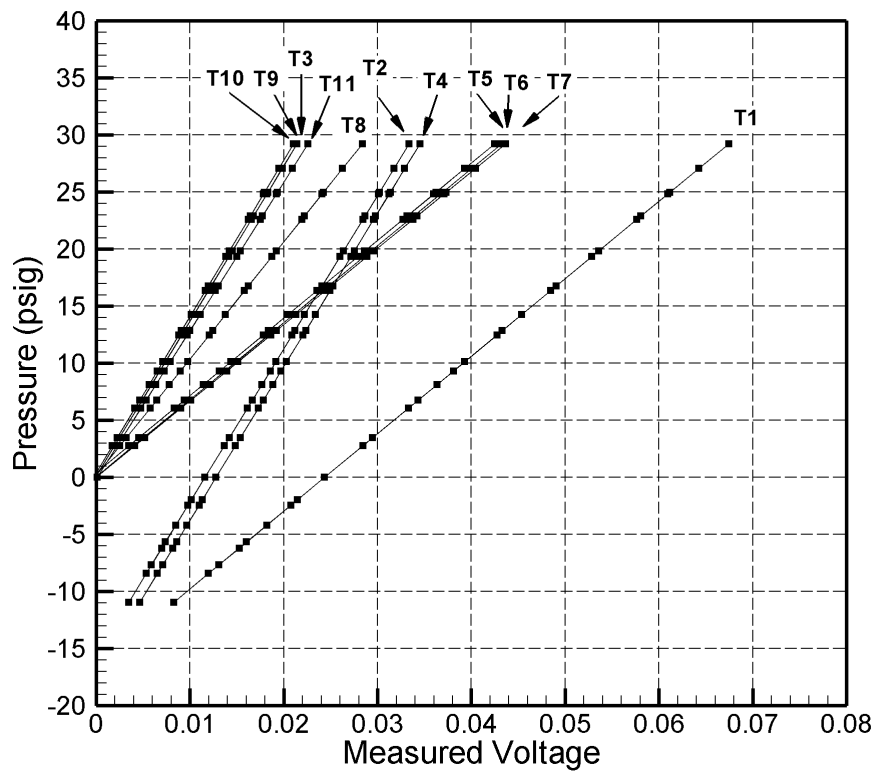

Figure 3.6: Transducer Calibration Curves

transducer. The pressure measured with this transducer is used to control the air compressor.

\subsection{Pressure Measurement Probes}

\subsubsection{Three Hole Pressure Probe}

Total pressure and flow angle are obtained using the three-hole probe (THP), the dimensions of which are shown in Figure 3.7. The probe was designed and manufactured by Islam (1999).

Three stainless steel hypodermic tubes with an outer diameter of $0.46 \mathrm{~mm}$ are soldered side by side to make the three measuring ports. The two outer tubes are bevelled at an angle of $30^{\circ}$ at the tip. The tip extends $5 \mathrm{~mm}$ from the probe body. The hypodermic tubing is housed in a stainless steel probe body that has a length of $165 \mathrm{~mm}$ and a diameter of $7 \mathrm{~mm}$ at its base. The body tapers to a diameter of $1.78 \mathrm{~mm}$ near the tip.

Pressure and flow angle measurements in unknown flow fields are obtained using the threehole probe by first calibrating the probe in a known flow. The calibration and data reduction 


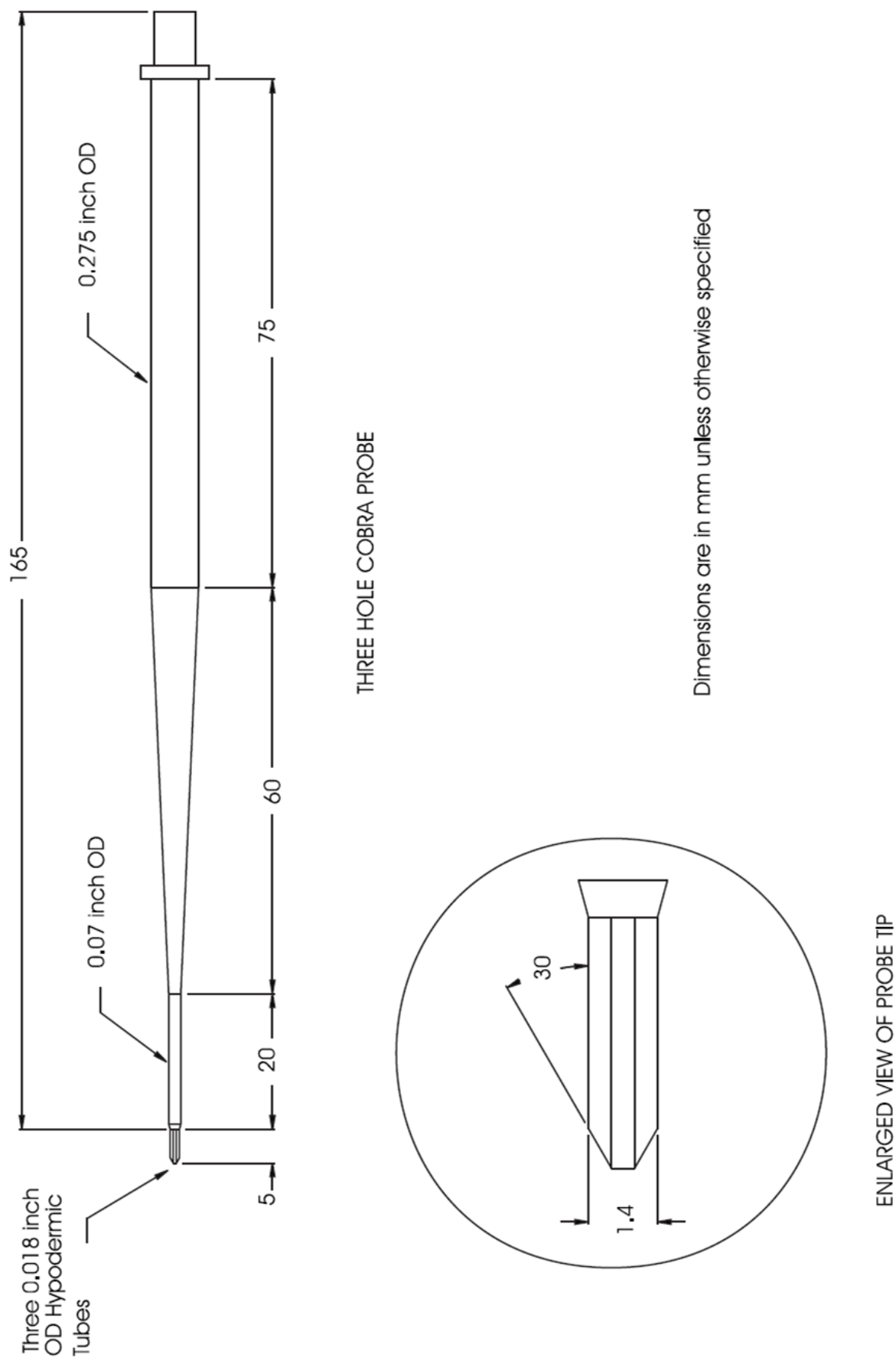

Figure 3.7: Three Hole Probe (Islam, 1999) 
procedures are described in Chapter 4. Currently, the calibration of three-hole probe is limited to subsonic Mach numbers. As will be seen, measurements in supersonic flow must, therefore, be corrected using the Rayleigh pitot formula. This requires measurements of the true static pressure, which are obtained using the static pressure probe.

\subsubsection{Static Pressure Probe}

Although it is possible to infer the static pressure from the three-hole probe data, in supersonic flows shocks form upstream of the probe tip. Without supersonic calibration data, the static pressure measured by the probe will not be the static pressure that would exist in the absence of the probe. Instead, the static pressure is obtained using the static pressure probe designed specifically for transonic flow. The dimensions of the static pressure probe, which was also designed and manufactured by Islam (1999), are shown in Figure 3.8 .

The conical tip of the static probe is bevelled at an included angle of $15^{\circ}$. The tip is designed to have negligible influence on flow. Two static pressure ports, $0.3 \mathrm{~mm}$ in diameter, are located $12 \mathrm{~mm}$ (roughly 12 probe diameters) from the tip and are positioned $180^{\circ}$ from one another. At the midspan of the cascade, the largest gradients in static pressure are in the pitchwise direction. Therefore, when installed in the test section, the two ports of the static probe are positioned vertically (i.e. the spanwise direction).

\subsubsection{Endwall Boundary Layer Probes}

Inlet boundary layer measurements are obtained using two boundary layer probes designed and manufactured by Corriveau (2005). The probes are designed with bent tips to allow measurements to be obtained very close to the wall. The dimensions are shown in Figure 3.9.

The flexible probe tips allow for the probes to touch the endwall without suffering damage. Placing the probe on the wall allows for the location of the probe to be known precisely. The procedure for measuring the endwall boundary layer is described in more detail by Corriveau (2005). For secondary flow investigations, detailed measurements of the endwall boundary layer are necessary. However, for the current study of profile losses, the inlet total pressure variation was measured simply to ensure spanwise uniformity at the midspan. Therefore, the 

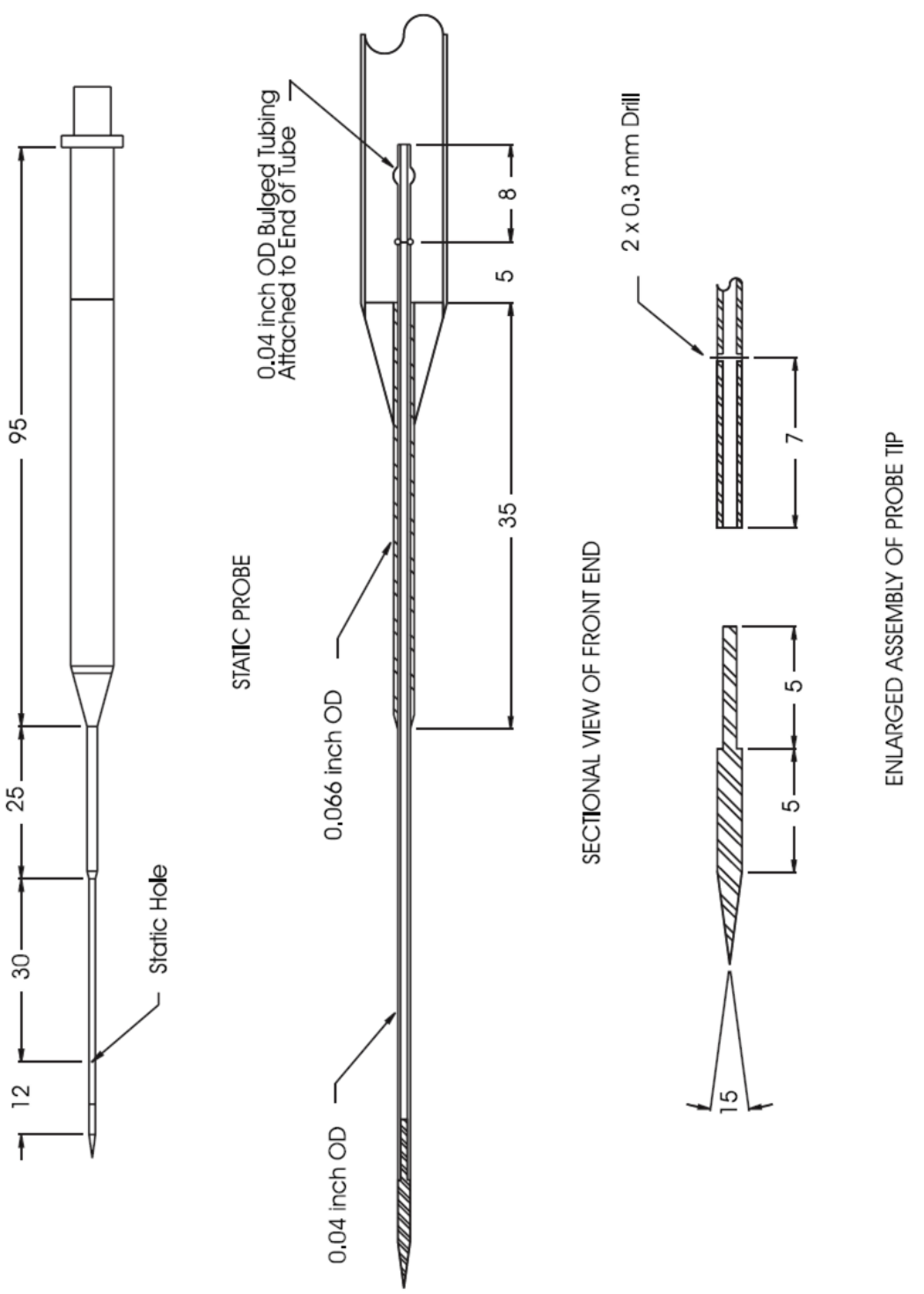

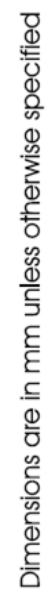

Figure 3.8: Static Pressure Probe (Islam, 1999) 


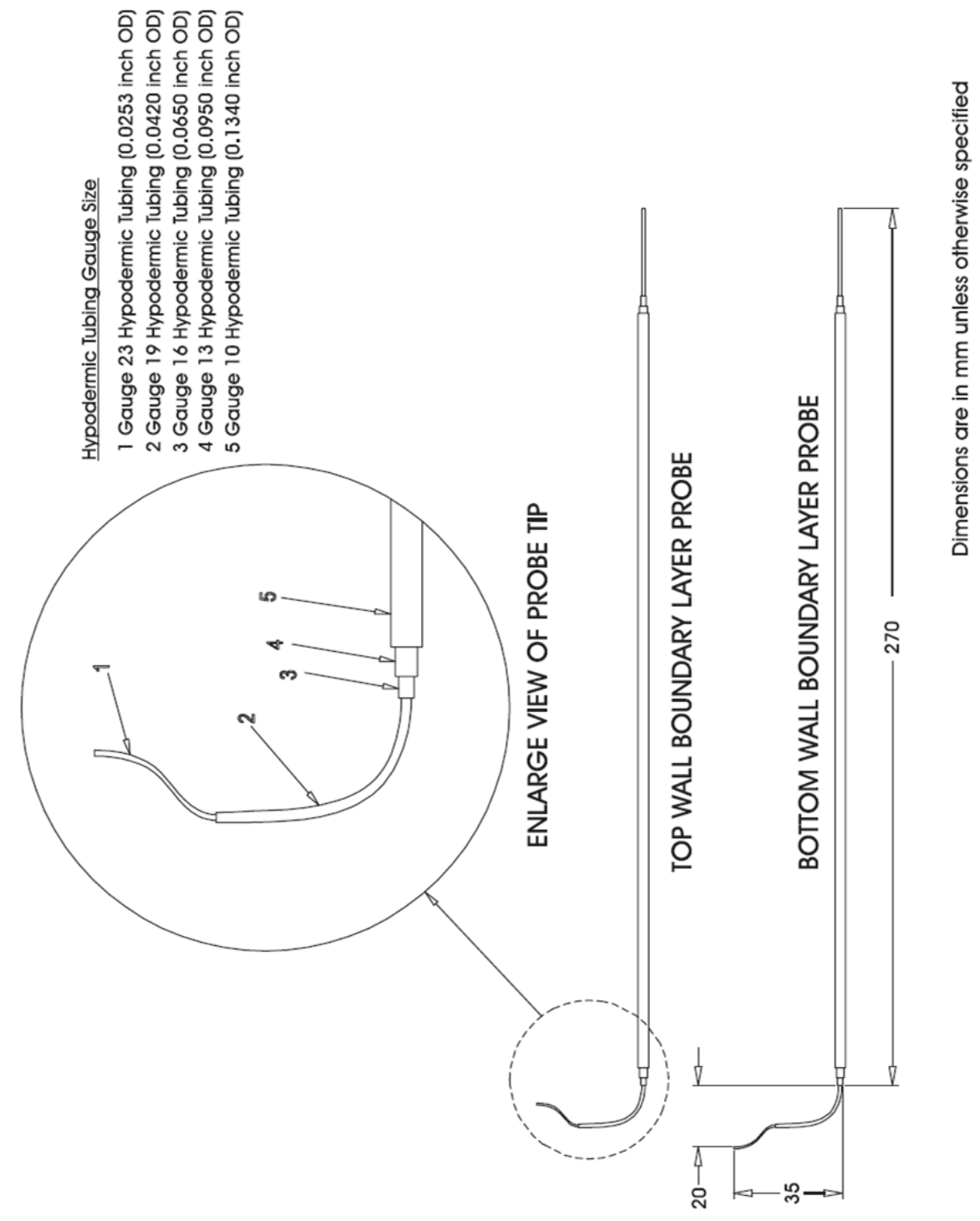

Figure 3.9: Boundary Layer Probes (Corriveau, 2005) 


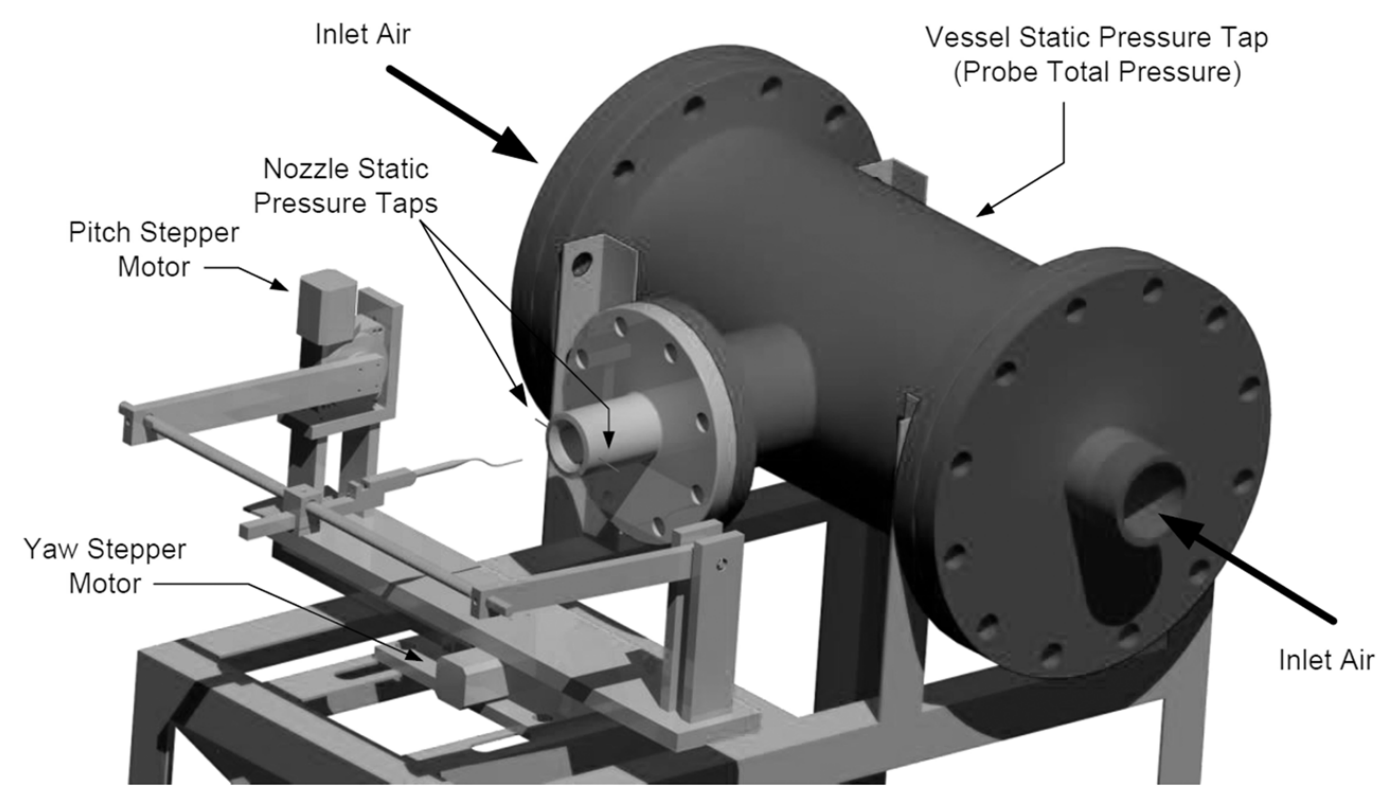

Figure 3.10: Probe Calibration Rig (Hall, 2012)

measurements did not extend to the wall. The inlet measurements will be further discussed in Chapter 4.

\subsection{Probe Calibration Rig}

To obtain accurate total pressure and flow angle measurements, the three-hole probe described in Section 3.4.1 must be calibrated. The calibration maps the characteristic of the probe over a range of yaw angles. The procedure for calibration is outlined in Section 4.3 . The probe was calibrated using the apparatus shown in Figure 3.10 ,

Pressurized air is supplied to the calibration rig settling chamber with the same flexible hoses that can be configured to supply air to the ejector-diffusor system. A 2 inch NelesJamesbury segmented-ball valve with a electro-pneumatic actuator is used to control the pressure in the settling chamber. The flow is accelerated through a bellmouth nozzle to a maximum exit Mach number of 1.0. When the probe is exactly aligned with the nozzle jet, the centre port measures the total pressure of the flow. This reading is used to correct the settling chamber pressure for the losses which occur in the nozzle. Therefore, the total pressure at 
the nozzle jet can be inferred from the settling chamber static pressure when the probe is misaligned with the jet. The alignment procedure is described in Section 4.3 .

The probe is mounted on a Velmex A5990TS rotary table capable of both pitch and yaw rotation. Rotation is achieved with the use of two Vexta PK245-01AA stepper motors capable of $1.8^{\circ}$ steps. The motors are geared so that the table can be moved in steps as small as $0.01^{\circ}$ with little backlash. The probe is positioned in such a way that the centre of rotation of the rotary table coincides exactly with the probe tip. Yaw and pitch angles can be varied between $-30^{\circ}$ and $30^{\circ}$.

Three-hole probes need only be calibrated in one direction, namely yaw. For measurements involving two flow directions, such as for endwall flow measurements, five- or seven-hole probes, which require calibration in both directions, are required.

\subsection{Cascade Overview}

\subsubsection{Geometry}

As mentioned in Chapter 1, the motivation for the current study came from Siemens Energy, Inc. In an effort to improve the profile loss prediction system used in their meanline analysis, the measurement of the profile losses of several turbine profiles was proposed.

The cascades used in this study were supplied by Siemens. The original intention was to measure the midspan flow for four different cascades. Profiles from the midspan and from near the tip (90\% of the blade span) from two Siemens engines, designated E1 and E2, were therefore supplied. The midspan (S50) cascades are designated E1S50 and E2S50, and the tip sections by E1S90 and E2S90. However, time constraints allowed for the flows of only three of the cascades to be measured: E1S90 was omitted and not manufactured. Key geometric and aerodynamic parameters are defined in Figure 3.11 and listed for the four profiles in Table 3.3. The dimensions for the airfoils have been scaled from the engine dimensions to appropriate sizes for the HSWT. Since E1S90 was not manufactured, it was not scaled. The profile coordinates for the Siemens cascades are given in Appendix A,

The HSWT has a channel height of $61 \mathrm{~mm}$. Corriveau (2005) showed that a blade aspect 
c: Chord length

$\mathbf{c}_{\mathbf{x}}$ : Axial chord length

i: Incidence angle

o: Throat opening

s: Pitch spacing

t: Trailing edge thickness

$\mathbf{W e}_{\mathbf{l e}}$ : Leading edge wedge angle

$\mathbf{W e}_{\mathrm{te}}$ : Trailing edge wedge angle

$\boldsymbol{\alpha}$ : Flow angle

B: Metal angle

$\boldsymbol{\delta}$ : Deviation angle

$\boldsymbol{\theta}_{\mathrm{UG}}$ : Unguided turning angle

$\zeta$ : Stagger angle

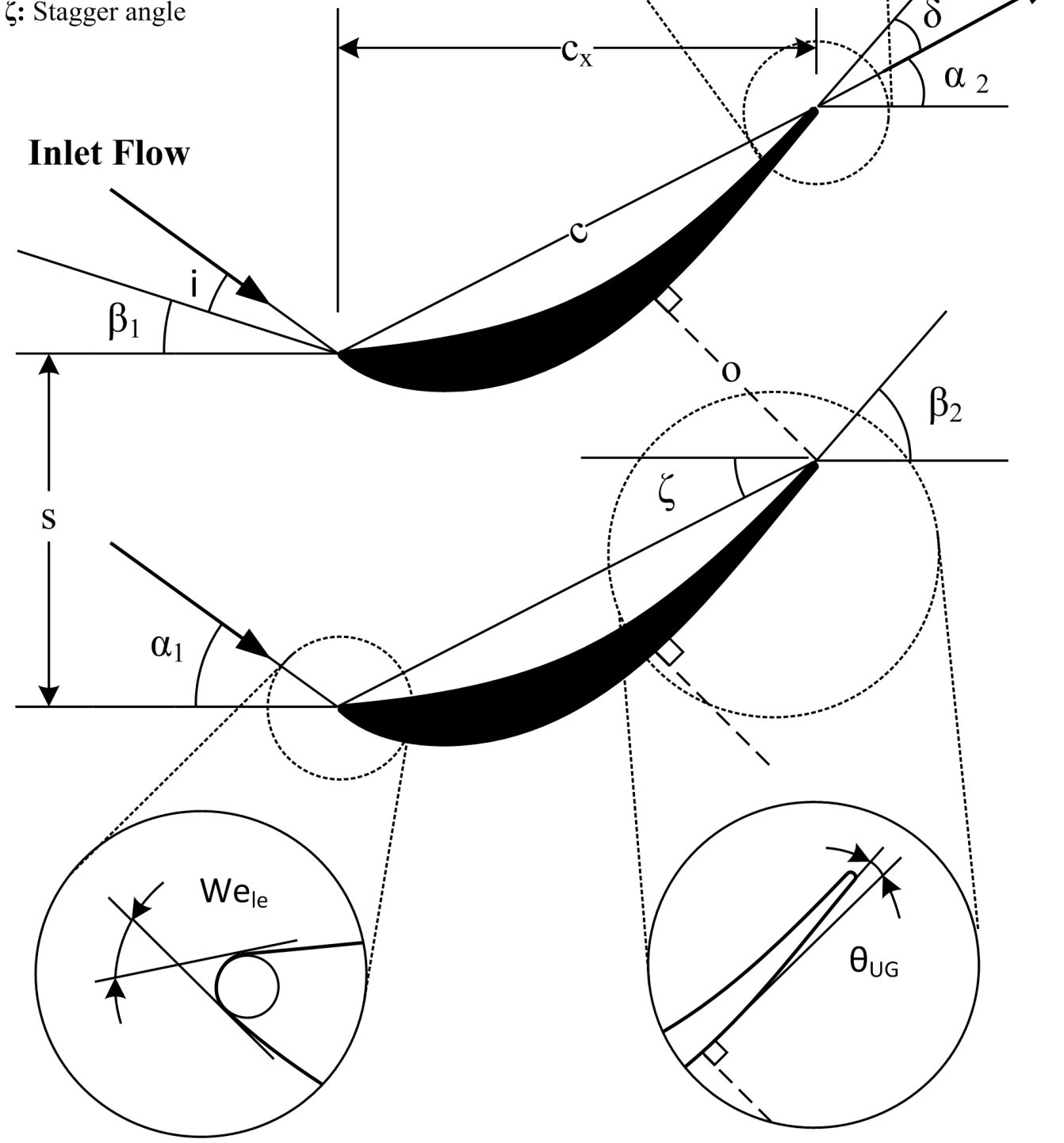

Figure 3.11: Symbols for Describing Airfoil Geometry 
Table 3.3: Cascade Geometry

\begin{tabular}{lcccc}
\hline & E1S50 & E1S90 & E2S50 & E2S90 \\
\hline \hline Chord Length, $c$ & $39.5 \mathrm{~mm}$ & - & $40.7 \mathrm{~mm}$ & $34.5 \mathrm{~mm}$ \\
Axial Chord, $c_{x}$ & $30.9 \mathrm{~mm}$ & - & $36.2 \mathrm{~mm}$ & $22.7 \mathrm{~mm}$ \\
Blade Span, $h$ & $61 \mathrm{~mm}$ & - & $61 \mathrm{~mm}$ & $61 \mathrm{~mm}$ \\
Blade Pitch, $s$ & $26.8 \mathrm{~mm}$ & - & $26.8 \mathrm{~mm}$ & $37.0 \mathrm{~mm}$ \\
Aspect Ratio, $h / c$ & 1.54 & - & 1.50 & 1.77 \\
Inlet Metal Angle, $\beta_{1}$ & $5.7^{\circ}$ & $-32.0^{\circ}$ & $17.8^{\circ}$ & $-12.6^{\circ}$ \\
Leading Edge Wedge Angle, $W e_{l e}$ & $88.8^{\circ}$ & $86.6^{\circ}$ & $56.6^{\circ}$ & $68.9^{\circ}$ \\
Outlet Metal Angle, $\beta_{2}$ & $58.0^{\circ}$ & $66.3^{\circ}$ & $49.0^{\circ}$ & $58.0^{\circ}$ \\
Trailing Edge Wedge Angle, $W e_{t e}$ & $3.4^{\circ}$ & $6.2^{\circ}$ & $5.7^{\circ}$ & $5.1^{\circ}$ \\
Trailing Edge Thickness, $t$ & $0.29 \mathrm{~mm}$ & - & $0.77 \mathrm{~mm}$ & $0.72 \mathrm{~mm}$ \\
Stagger Angle, $\zeta$ & $38.6^{\circ}$ & $59.5^{\circ}$ & $27.1^{\circ}$ & $48.9^{\circ}$ \\
Unguided Turning, $\theta_{U G}$ & $4.6^{\circ}$ & $9.5^{\circ}$ & $9.1^{\circ}$ & $13.7^{\circ}$ \\
Throat Opening, $o$ & $13.7 \mathrm{~mm}$ & - & $17.0 \mathrm{~mm}$ & $20.6 \mathrm{~mm}$ \\
Pitch-to-Chord ratio, $s / c$ & 0.678 & 0.843 & 0.658 & 1.072 \\
Trailing Edge Blockage, $t / o$ & 0.021 & 0.024 & 0.045 & 0.035 \\
Number of Blades, $N$ & 7 & - & 7 & 5 \\
\hline
\end{tabular}



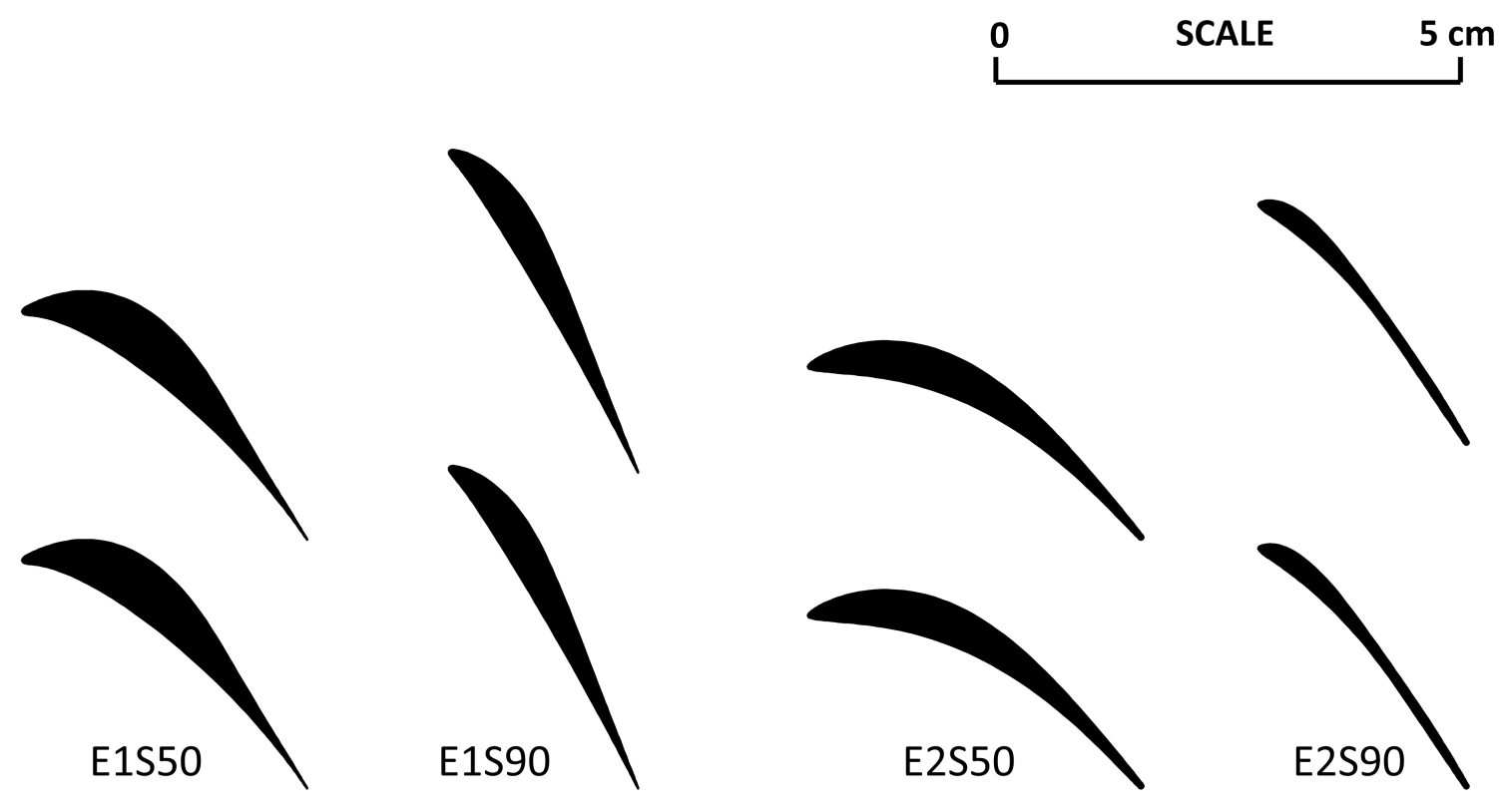

SCALE

$5 \mathrm{~cm}$

Figure 3.12: Siemens Cascade Profiles

ratio, $h / c$, of at least 1.5 is sufficient to allow for a region of essentially two-dimensional flow at the midspan, which is essential for measurements of profile losses. Therefore, the chord length of the blades was chosen with consideration for this requirement. Furthermore, the two midspan cascades were sized such that the pitch spacing was the same. This allowed for the same mounting plate to be used for the two cascades. Finally, due to the low solidity of E2S90, the tip section cascade used only five blades. Limiting the cascade to five blades kept the total throat area low enough to ensure adequate run times at high Mach numbers.

The four profiles are shown with correct proportions in Figure 3.12 . The scale is representative of the cascades as they where manufactured for testing in the HSWT. Although E1S90 was not manufactured, the representation in Figure 3.12 is included for comparison with the other profiles and since its predicted losses are discussed in Chapter 5 .

\subsubsection{Manufacturing}

The Siemens cascades were manufactured by Forecast3D in Carlsbad, CA, and Direct MFG, Inc. (DMI) in Austin, TX. The blades were manufactured using a direct metal laser 
sintering (DMLS) rapid prototyping process. In this additive process, the blade is sintered in $0.02 \mathrm{~mm}$ layers from a powdered form of the alloy of choice. The part is built in an inert atmosphere and each layer is sintered using a high power laser. Following the completion of a layer, the part is lowered and $0.02 \mathrm{~mm}$ of powdered metal is deposited over the previous layers. For the current blades, cobalt-chrome MP1 was the material selected. This super-alloy provided the material properties to achieve the necessary manufacturing tolerances required for high-speed cascade testing. Tight tolerances are particularly important for the current study due to the very thin trailing edges of the Siemens cascades. Furthermore, cobalt-chrome MP1 was suggested by the manufacturers to limit bowing of the thin trailing edges that was observed in early trial parts.

The midspan cascade E2S50 was the first blade to be manufactured and served as a trial for the process. It was found that the DMLS process resulted in a surface roughness of about $10 \mu \mathrm{m}$. This was considered too high and consequently several blades were chemically polished. It was found that this polishing process removed about $0.25 \mathrm{~mm}$ (0.01 inches) of material from all surfaces. For E2S50, this resulted in a change in trailing edge thickness of the order of $30 \%$. Therefore, the rough blades were used for the loss measurements for E2S50. For subsequent cascades, the profiles were manufactured with additional thickness to allow for polishing. After manufacturing and polishing, the blades were then inspected. Unfortunately, due to the manner in which the blades were polished, the amount of material removed was not completely consistent across the blade span; slightly more material was removed from the tips of the blades than from the base. Furthermore, slight variations were noted between blades in the cascade. It is not clear whether this had any effect on flow periodicity or loss measurements. Further discussion of flow quality follows in Chapter 4.

Laser sintering offers an advantage in regard to manufacturing time and cost when considering blades with surface static taps. The sintering process allows for internal features to be built into the blades during the sintering process eliminating the need to drill the taps after the blades are manufactured. Furthermore, this reduces the effect the static taps will have on the flow behaviour since drilling can cause raised edges around the static tap. Due to the thinness of the Siemens cascades, only E2S50 could be instrumented with static taps. 

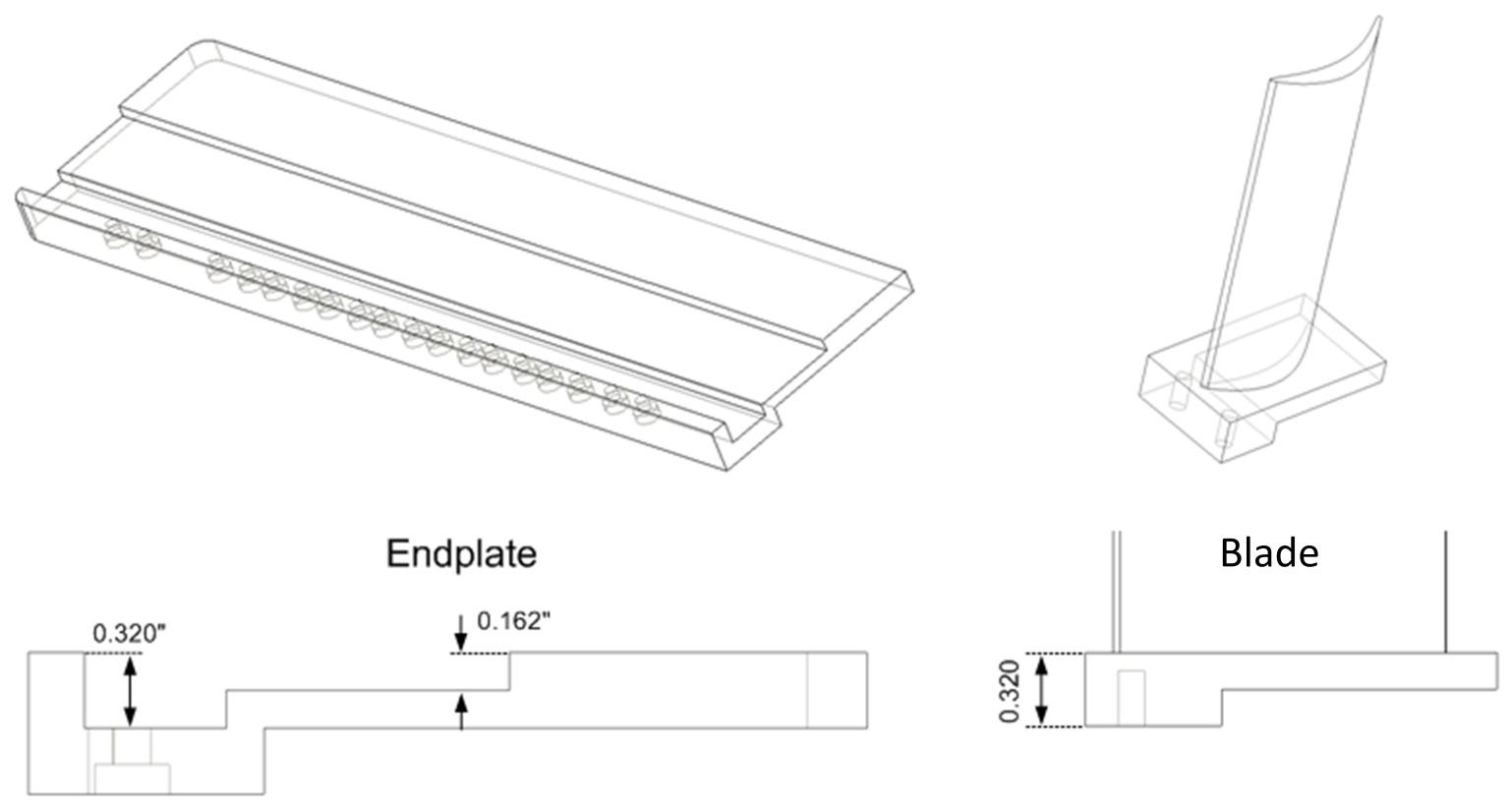

Figure 3.13: Cascade Mounting: Endplate and Mounting Platform

The benefit to traditionally machining and drilling instrumented blades is the quality of the surface finish and the static taps. Static taps produced by sintering are not perfectly round, and in some cases, the roughness produced by the sintering may partly obstruct the static tap. Imperfections in static taps can lead to some errors in pressure measurements. Furthermore, the chemical polishing process affects the size and shape of the static taps. However, these effects are likely small and within the uncertainty in the measurements.

\subsubsection{Cascade Assembly}

Due to the thinness of the current blades, the method by which past blades were mounted in the test section, as described by Corriveau (2005), could not be used to mount the current blades. In previous studies, the stagger angle was set with two dowels and the blades were mounted with a bolt in the hub and tip. For the Siemens cascades, the blades were manufactured with an attached platform that is mounted in the endplate. The platforms set the stagger angle and the pitch spacing of the blades. The endplate and platform are shown in Figure 3.13 . 


\section{Chapter 4}

\section{Experimental Procedures and Measurement Uncertainties}

\subsection{Introduction}

Although measurements similar to those obtained in this study have been made in the past using the same facility, all of the details of the data reduction procedures have not previously been documented in one place. Furthermore, as components of the wind tunnel have been updated and improved, the experimental procedures change. Therefore, this chapter will describe all of the current procedures used for measuring the midspan flow of turbine cascades in the Carleton high-speed wind tunnel.

First, the data acquisition and control system will be described. The methods by which measurements are obtained and processed are included. A description of the three-hole probe calibration procedure follows. Reduction of the three-hole probe data, application of the Rayleigh pitot correction, and mixing-out calculations for non-uniform flows are also described. A brief description of the measurement uncertainty calculations is then given and the values of the uncertainties are presented. The chapter concludes with a discussion of the flow quality assessment. Inlet spanwise and pitchwise flow uniformity, and outlet flow periodicity for the Siemens cascades are presented. 


\subsection{Data Acquisition and Control System}

\subsubsection{Measurement Locations}

Figure 4.1 shows a scale drawing of the E2S50 midspan cascade as mounted in the test section. All measurement locations are given relative to the cascade dimensions. Measurements for E1S50 and E2S90 follow the same scheme. However, as mentioned in Chapter 3 , cascade E2S90 has only five blades.

As mentioned in Chapter 3 a reference inlet total pressure $P_{o, r e f}$, and total pressure $T_{o}$, are measured far upstream of the cascade. Since entropy is generated between the reference measurement location and the inlet to the cascade, the reference pressure must therefore be corrected to obtain the value at the cascade inlet. The total pressure is measured on the centerline about $40 \mathrm{~mm}$ upstream of the cascade leading edge for all operating Mach numbers. These measurements are then used to correct the reference pressure for subsequent tests. The value of $P_{o 1} / P_{o, r e f}$ varies between 0.995 and 0.998 over the range of isentropic exit Mach numbers from 0.8 to 1.3 .

Spanwise measurements of the total pressure are also obtained on the centreline $40 \mathrm{~mm}$ upstream of the leading edge. These measurements are used to determine the thickness of the inlet endwall boundary layer.

The static taps shown upstream and downstream of the cascade are drilled into the Lexan window that seals the top of the test section. The taps have a diameter of $0.4 \mathrm{~mm}$ and are spaced by about 0.5 pitch spacings for each cascade. The taps are used to measure the static pressure at the endwall $1.0 c_{x}$ upstream and $1.5 c_{x}$ downstream of the leading edge.

The static pressure upstream of the cascade, $P_{1}$, is assumed to be constant across the span. The endwall static pressure is therefore taken to be the static pressure at the midspan. Pitchwise measurements of static pressure, $P_{1 y}$, are obtained across the inlet for each cascade at each value of inlet flow angle to ensure that there is no static pressure gradient in the pitchwise direction. However, only those ports labeled " $P_{1}$ " in Figure 4.1 are measured concurrently with the downstream wake measurements. The upstream static pressure is obtained by averaging the four ports using a manifold and a single transducer is used for the 


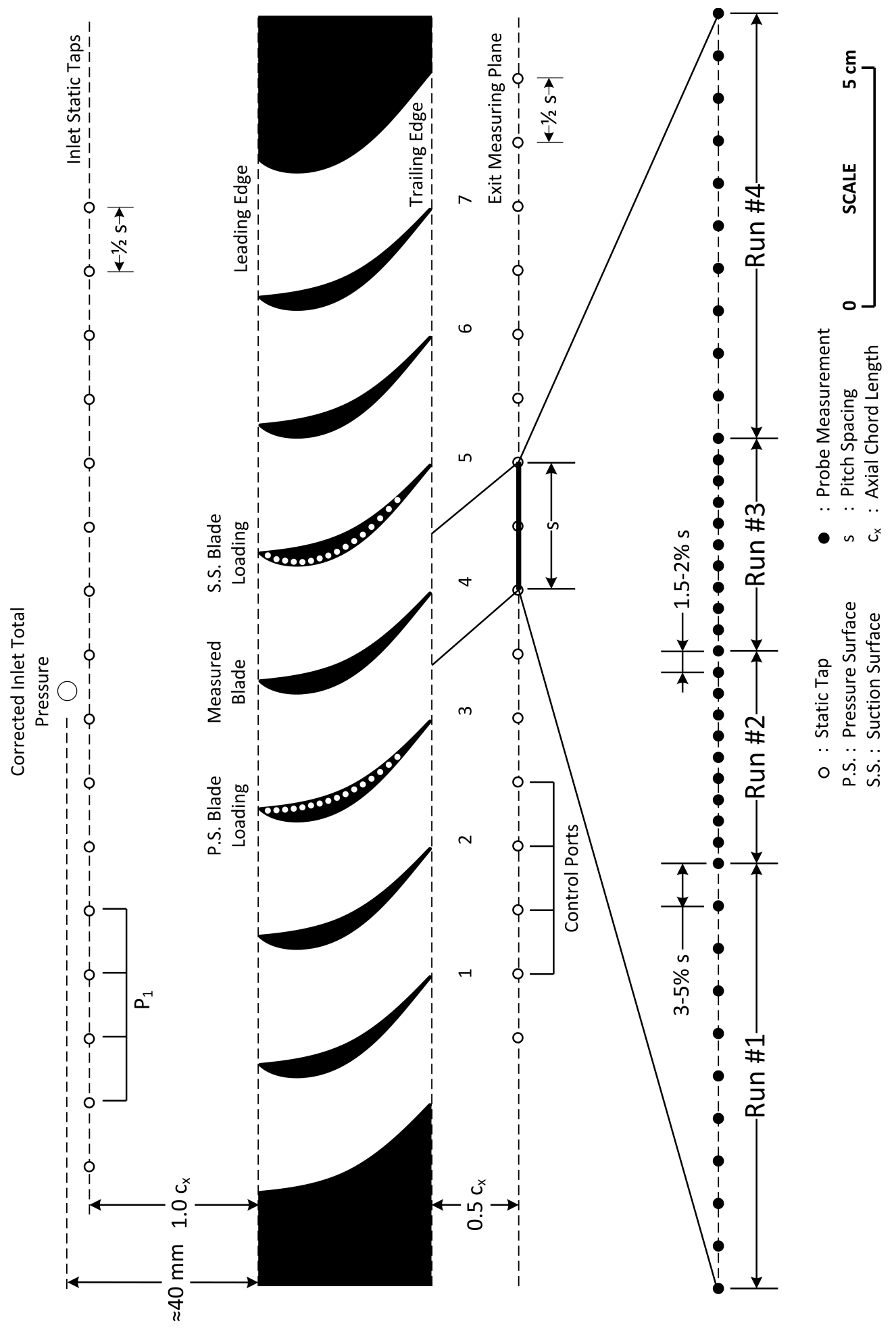

Figure 4.1: Measurement Locations 
measurement.

Similarly, the static pressure measurements from all of the downstream static taps are recorded for each operating point. These measurements require much less time to obtain than probe traverse measurements and thus serve as a first approximate measure of the outlet flow periodicity. The static pressure measurements obtained by the four ports labeled "control ports" in Figure 4.1 are averaged and measured by a single transducer. This is the downstream static pressure used by the control system to control the wind tunnel operating Mach number. The control system will be discussed further in Section 4.2.4.

Figure 4.1 shows the locations of the blade loading measurements for E2S50. The centre blade is not instrumented to avoid error in the downstream measurements for that blade. The instrumented blades are positioned in such a way that the loading measurements are obtained for the shared flow passage between the centre blade and the instrumented blade. Blade loading measurements will be presented in Chapter 5.

The three-hole probe and static pressure probe measurements are obtained at a location $1.5 c_{x}$ downstream of the leading edge. To asses the outlet flow periodicity, the five centre blades are traversed. Thus, for the tip cascade E2S90, measurements are obtained for the entire cascade. However, for the detailed measurement of the midspan flow, only the centre blade is traversed. The pitchwise locations at which probe measurements are obtained for the detailed wake traverses are shown in Figure 4.1. 41 data points are obtained over one pitch and are typically collected over a total of four runs. However, at subsonic exit Mach numbers it is sometimes possible to obtain the 41 data points in fewer than four runs. Regardless of the number of runs required, the measurements are always spaced more closely over the middle third of the pitch to capture the large gradients in total pressure and flow angle present in the blade wake. Flow quality and periodicity measurements will be discussed further in Section 4.6 and the centre blade results in Chapter 5.

\subsubsection{Data Acquisition System}

The data acquisition system for the HSWT was updated by Taremi et al. (2008) as part of an effort to extend the wind tunnel capabilities to include the measurements of endwall flows. 
The data acquisition system was updated to improve the response time of the old system. The data acquisition is managed with an in-house program developed using LabVIEW8.

The voltages produced by the pressure transducers are amplified and recorded using a 16-bit National Instruments (NI) PCI-6229 data acquisition card installed in a Microsoft Windows-based PC computer. Similarly, the voltage produced by the K-type thermocouple is amplified, filtered and acquired using a 24-bit National Instruments USB-9211A data acquisition unit installed in the same PC.

To reduce errors introduced through turbulent fluctuations and operating point drift, the signals are sampled at a frequency of $2000 \mathrm{~Hz}$ and averaged over a 0.1 second period. Hence, 200 measurements are averaged for a single data point. Further discussion of the sampling rate and data integration time follows.

\subsubsection{Data Sampling Rates and Integration Times}

The dynamic characteristics of the HSWT were investigated by Jeffries et al. (1997). Both the low and high frequency characteristics were described. The goal of the investigation was to determine suitable data sampling rates and integration times for pressure measurements.

The low frequency characteristics showed that the pressure measurements fluctuate by about $1 \%$ of the mean value with a frequency of about $0.5 \mathrm{~Hz}$. The source of this fluctuation was determined to be the wind tunnel control system. Although the control system has been redesigned by Hall (2012), the low frequency characteristics have not since been measured.

The high frequency fluctuations, which are caused by inherent turbulence in the flow, were measured to have a frequency of the order of $1000 \mathrm{~Hz}$. Following the Nyquist criterion, a sampling rate of $2000 \mathrm{~Hz}$ was chosen. At $2000 \mathrm{~Hz}$ it was found that an integration time of 0.1 seconds was sufficient to obtain a pressure measurement with a relative error of less than $1 \%$.

To correct for the low frequency fluctuations, Jeffries et al. suggest normalizing all pressures by the blowing pressure. The blowing pressure measurements will tend to fluctuate roughly in phase with the other measurements due to the proximity at which all measurements are obtained. However, variation in blowing pressure, and hence Mach number, leads 
to small variations in losses. This is particularly true at transonic conditions where shock locations and strengths are sensitive to changes in Mach number. Therefore, normalizing by upstream total pressure may not, in some cases, remove all fluctuations in downstream parameters which are dependent on losses.

In addition to the sampling rates and integration times suggested by Jeffries et al. (1997), the plumbing between the transducers and the static taps and pressure probes is kept as short as possible to reduce the total volume of the pressure sensing system.

\subsubsection{Control System}

The HSWT control system was redesigned by Hall (2012). Hall integrated the control system software with the data acquisition software described previously. The NI PCI-6229A card, which acquires the transducer voltage signals, can also generate output voltage signals. As mentioned in Chapter 3 , the operating point of the wind tunnel (i.e. the cascade exit Mach number) is controlled with a Neles-Jamesbury 4 inch ball valve positioned by a current-to-air electro-pneumatic actuator. Therefore, the voltage signal must be converted to a current signal. The voltage supplied by the NI PCI-6229A varies between 0 and 10 volts and is converted to amperage using an Omega OM7-39-02-C voltage-to-current convertor which supplies the necessary $0-20 \mathrm{~mA}$ of current to the actuator.

Since the aim is to achieve constant Mach number, both the upstream total pressure $P_{o 1}$ and the downstream static pressure $P_{2}$ measured at the endwall are sampled by the control system using the data acquisition system described above. The calibration curves for each of the transducers is used to convert the measured voltage to a pressure. Although the slope of these curves is consistent, the offsets have been known to drift over time. Therefore, new values for the offsets are measured daily and entered into the control system as part of the start-up routine. The exit isentropic Mach number $M_{2, i s}$ can be calculated from $P_{o 1}$ and $P_{2}$ and used for control. A feedback P.I.D. control system adjusts the voltage that is supplied to the control valve, thus adjusting the blowing pressure and Mach number.

A disadvantage to controlling on the isentropic Mach number is that the relationship between isentropic Mach number and true Mach number is dependent upon the losses. Therefore, 
it becomes difficult to obtain results at a specific intended Mach number when other parameters, such as incidence are also varied since the losses will also change. The actual Mach number obtained is therefore determined after the measurements have been completed.

\subsection{Three-Hole Probe Calibration}

The three-hole probe measurements in the current study have been obtained while using the probe described in Section 3.4.1 in non-nulling mode. That is, the probe is set to a fixed angle in the wind tunnel and measurements of flow angle are made relative to the probe orientation. Probes which are used in this manner must first be calibrated in a flow of known direction. This section describes the calibration procedure and presents the calibration curves for the Carleton three-hole probe (THP). Probe calibration was performed most recently by the current author for high subsonic Mach numbers, and by Hall (2012) for lower subsonic Mach numbers. Calibration curves were obtained using the calibration rig described in Section 3.5. which can achieve a maximum Mach number just below 1.0. As mentioned in Chapter 3. the probe is mounted in such a way that the location of the probe tip is held at a fixed position within the calibration jet as the yaw or pitch angles are varied.

Before calibration, the probe axis must first be aligned with the calibration jet. Alignment is done aerodynamically. Figure 4.2(a) shows the probe orientation and the relevant angles. $\theta$ is the angle of the calibration jet with respect to the zero-yaw reference of the calibration rig and varies with the Mach number. Therefore, the alignment procedure must be repeated for each Mach number at which the probe is calibrated.

A perfectly symmetrical probe will be aligned with the jet when the two side ports measure equal pressures (i.e.: $P_{\text {port } 2}-P_{\text {port } 3}=0$ ). Hence for a symmetric probe, the yaw angle $\alpha$ at alignment will be equal to $\theta$. However, it is impossible to manufacture perfectly symmetrical probes. Consequently, if the bevel angle $\beta$ differs between the two sides by an angle $\beta_{e}$, as shown in Figure 4.2(a), the measured pressures from these ports will be equal when $\alpha=\theta+\frac{\beta_{e}}{2}$.

To determine the direction of the flow vector (i.e. $\theta$ ) as well as the asymmetry of the probe $\beta_{e}$, the difference between the two side port pressures is plotted over a range of yaw 


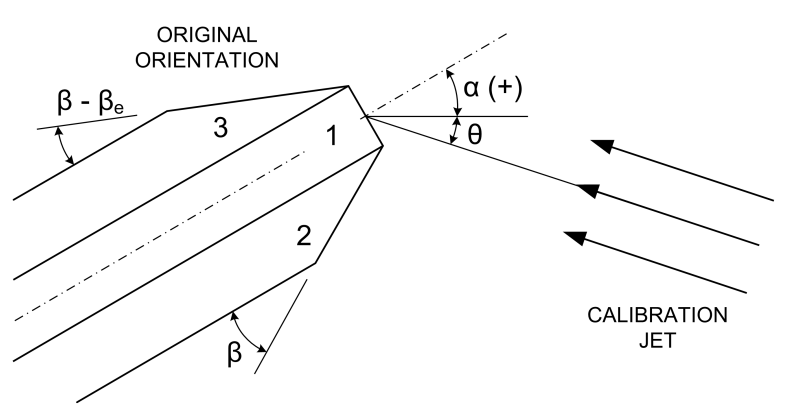

(a) Probe Geometry and Flow Angles

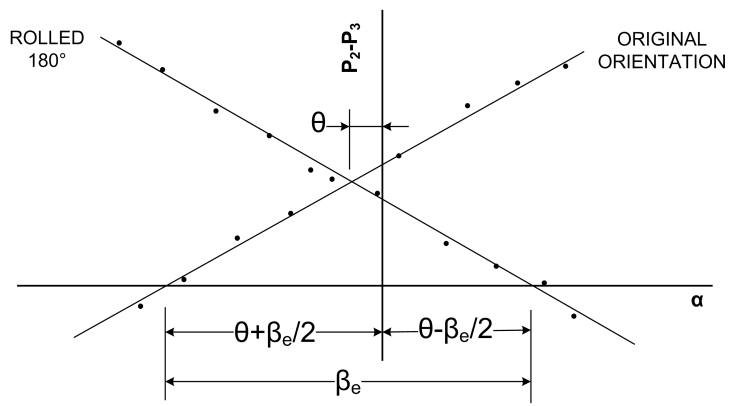

(b) Aerodynamic Influence of Asymmetry and Misalignment

Figure 4.2: Three-Hole Probe Nomenclature

angles. The probe is then rolled by $180^{\circ}$ in the calibration rig and the procedure repeated. In the rolled orientation, the side port pressures will now be equal at the yaw angle, $\alpha=\theta-\frac{\beta_{e}}{2}$. A hypothetical example of this is shown in Figure 4.2(b). It follows that $\theta$ will correspond to the point of intersection of the two calibration curves. Furthermore, $\beta_{e}$ can be determined from the difference in yaw angles at which $P_{\text {port } 2}-P_{\text {port } 3}=0$ between the two orientations. Once the direction of the calibration jet is determined, the probe is rolled $180^{\circ}$ back to the original orientation and aligned with the jet. Figure 4.3(a) shows that the asymmetry of the three-hole probe is of the order of $0.25^{\circ}$, which as will be discussed further, is within the uncertainty of the measurements.

As mentioned in Chapter 3, once the probe is aligned with the calibration jet the centre port effectively acts as a pitot tube. Therefore, the pressure measured by this port is the total pressure of the jet. This measurement is used to calibrate the calibration rig itself for the losses which occur in the nozzle. The static pressure measured in the settling chamber can then be used to infer the total pressure in the jet for subsequent calibration points, at which the probe may be at some yaw angle other than zero. Using two static taps that measure the pressure at the nozzle exit the Mach number of the jet can be calculated. Similar to the test section control system, the calibration control system controls the Mach number of the calibration jet.

Calibration coefficients that vary with yaw angle, $\alpha$ are calculated based on the three port 
pressures $\left(P_{\text {port } 1}, P_{\text {port } 2}\right.$ and $\left.P_{\text {port } 3}\right)$, and are defined as follows:

$$
\begin{gathered}
C_{\alpha}=\frac{P_{\text {port } 2}-P_{\text {port } 3}}{P_{\text {port } 1}-\bar{P}} \\
C_{o}=\frac{P_{o}-P_{\text {port } 1}}{P_{\text {port } 1}-\bar{P}}
\end{gathered}
$$

and

$$
C_{s}=\frac{P_{o}-P_{s}}{P_{\text {port } 1}-\bar{P}}
$$

where, $P_{s}$ is the static pressure measured at the nozzle exit, $P_{o}$ is the total pressure inferred from the settling chamber pressure and

$$
\bar{P}=\frac{P_{\text {port } 2}+P_{\text {port } 3}}{2}
$$

The calibration coefficients are calculated over a range of yaw angles from $-15^{\circ}$ to $15^{\circ}$ in $0.5^{\circ}$ steps at each Mach number. Following the same procedure as Hall (2012) to reduce scatter, the calibration is repeated five time and a best fit curve is then applied to the average values of the coefficients. The result is an expression for each coefficient as a function of yaw angle at each Mach number. These expressions are then used to generate a table of calibration coefficients at angles in $0.5^{\circ}$ intervals. For the current study, calibration tables were generated for the three-hole probe at the following Mach numbers: 0.79, 0.89 and 0.98 . However, calibration tables were generated by Hall (2012) at Mach numbers as low as 0.2. Calibration tables can then be generated at intermediate Mach numbers by linearly interpolating between two original tables. Relevant calibration curves are shown in Figures 4.3(b), 4.3(c), and 4.3(d),

Currently, the calibration rig is limited to Mach numbers below 1.0. When the probe is used to measure an unknown supersonic flow, a shock will form upstream of the probe tip. However, static pressure data, obtained from the transonic static pressure probe described in Section 3.4.2, provides the necessary information to solve the Rayleigh pitot formula and therefore correct the three-hole probe measurements. The Mach number behind the shock is likely to be subsonic. Therefore, the calibration curves should be adequate for determining flow angle, particularly since at midspan the probe is always aligned with the flow direction to within about $5^{\circ}$. The Rayleigh pitot correction will be described in Section 4.4.2. 


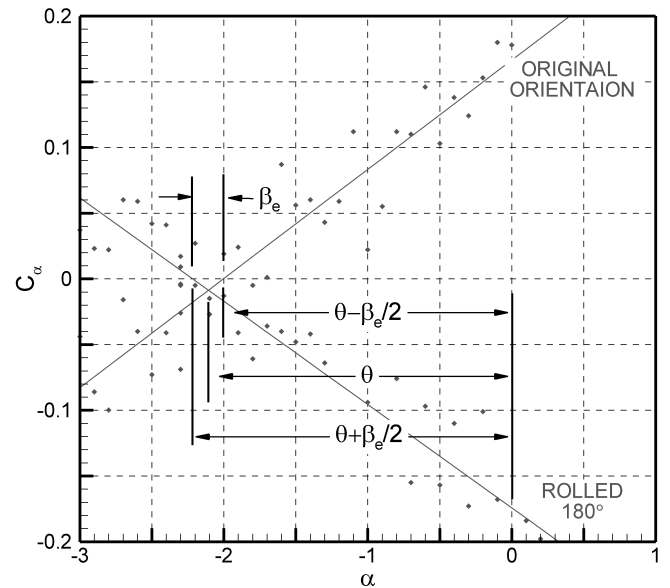

(a) Probe Alignment and Asymmetry

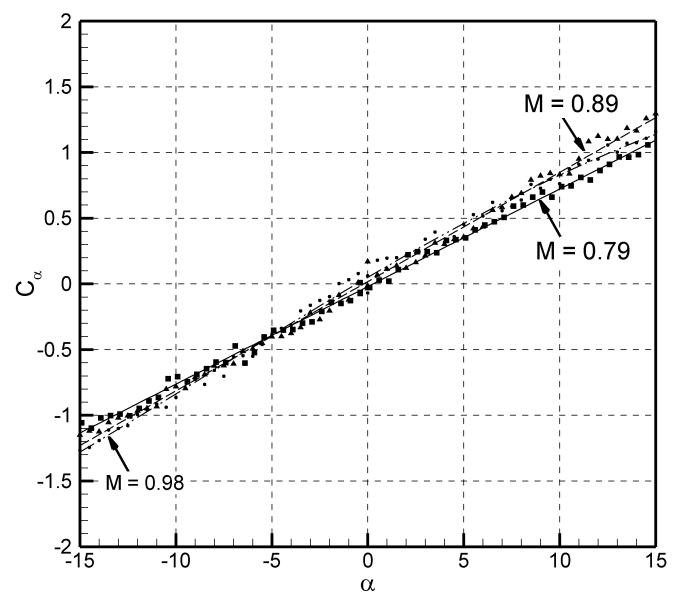

(c) Variation of $C_{\alpha}$

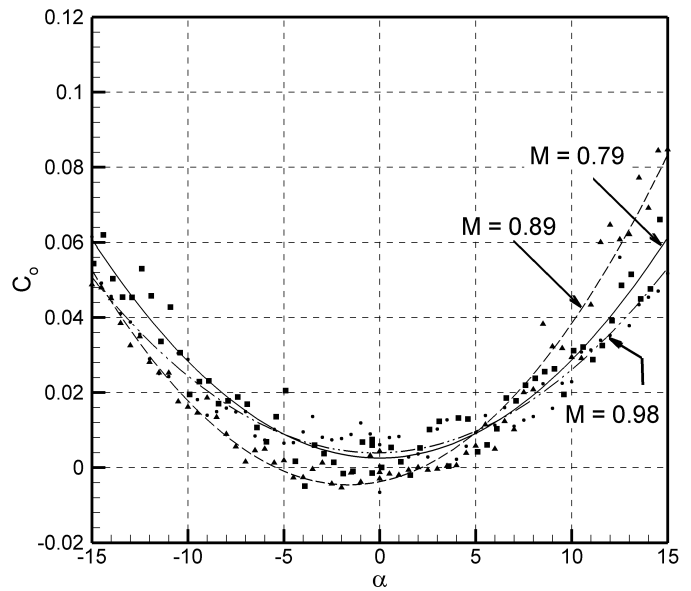

(b) Variation of $C_{o}$

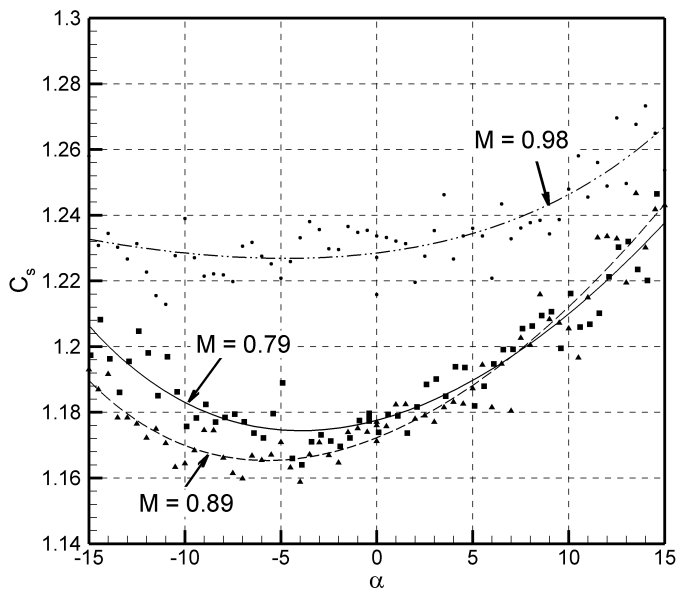

(d) Variation of $C_{s}$

Figure 4.3: Thee-Hole Probe Calibration Curves 


\subsection{Data Reduction Procedures}

\subsubsection{Three-Hole Probe Data Reduction}

To obtain measurements of total pressure, static pressure, and flow angle in the unknown flow field downstream of the cascade, the three-hole probe data must be processed using the calibration curves discussed previously. Additionally, measurements from the static pressure probe must be combined with the three-hole probe data and the alignment between probes must be verified.

The calibration coefficient $C_{\alpha}$ can be calculated from Equation 4.1 with the three port pressures measured by the three-hole probe. The value of $C_{\alpha}$ can then be used to find the flow angle $\alpha$ in the appropriate calibration table. To determine which calibration table is to be used, the Mach number must first be estimated. A new calibration table is generated by interpolation for Mach numbers which fall between the existing tables. No extrapolation is performed if the Mach number falls outside the range of calibration data. Instead, the highest or lowest Mach number table is used. However for exit Mach numbers above 1.0, the total pressure must also be corrected using the Rayleigh pitot formula which will be described further in Section 4.4.2. The flow angle is then used to determine both $C_{o}$ and $C_{s}$ from the calibration tables, which in turn yield estimates for the total and static pressure. However, the static pressure reduced in this way is not used for further calculations. The total pressure reduced from the three-hole probe and static pressure from the static pressure probe are used to calculate a new estimate for the Mach number. The procedure is then repeated until the calculated Mach number corresponds to the Mach number for the interpolated calibration tables.

\subsubsection{Rayleigh Pitot Correction}

At supersonic conditions, a bow shock will form upstream of the probe tip due to the presence of the probe as indicated in Figure 4.4. Consequently, the three hole probe measures a lower total pressure than would exist in the absence of the probe. Fortunately, the shock just upstream of the probe is normal and the pressure loss across the shock is a well-defined 


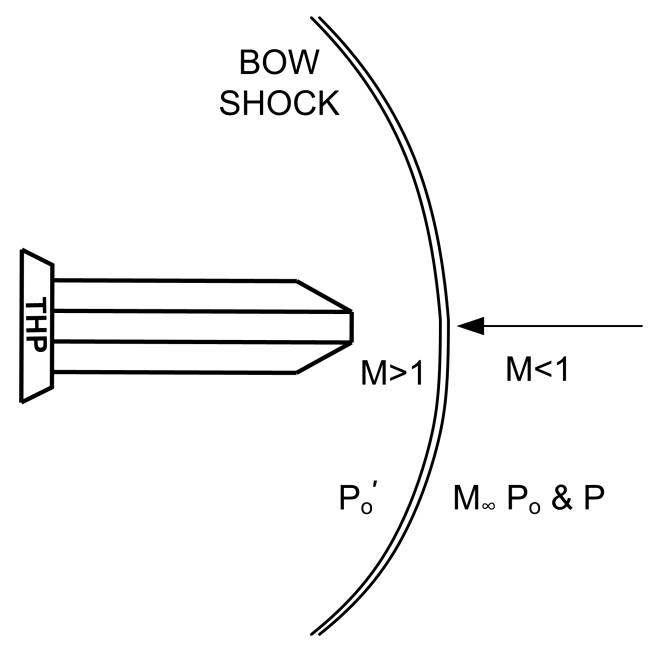

Figure 4.4: Three Hole Probe Bow Shock

function of the upstream Mach number.

Considering the schematic of the probe tip and shock shown in Figure 4.4, the total pressure change across the shock is defined by,

$$
\frac{P_{o}^{\prime}}{P_{o}}=\left[\frac{(\gamma+1) M_{\infty}^{2}}{(\gamma-1) M_{\infty}^{2}+2}\right]^{\frac{\gamma}{\gamma-1}}\left[\frac{\gamma+1}{2 \gamma M_{\infty}^{2}-(\gamma-1)}\right]^{\frac{1}{\gamma-1}}
$$

where $\gamma$ is the specific heat ratio, $P_{o}^{\prime}$ is the total pressure downstream of the shock (measured by the three hole probe), and $P_{o}$ and $M_{\infty}$ are the true total pressure and Mach number upstream of the shock (White, 2006).

To find the true Mach number, the static pressure $P$ is measured separately using the static pressure probe. As mentioned in Chapter 3 , the static pressure probe is designed to measure true static pressure at transonic conditions. With the true static pressure known, the true Mach number can then be found using the Rayleigh Pitot Formula as follows:

$$
\frac{P_{o}^{\prime}}{P}=\left[\frac{(\gamma+1) M_{\infty}^{2}}{2}\right]^{\frac{\gamma}{\gamma-1}}\left[\frac{\gamma+1}{2 \gamma M_{\infty}^{2}-(\gamma-1)}\right]^{\frac{1}{\gamma-1}}
$$

The Rayleigh Pitot formula can be solved iteratively for Mach number. Once the Mach number is determined, the total pressure ratio across the shock can then be calculated with Equation 4.5 and then the undisturbed total pressure $P_{o}$ determined. 


\subsubsection{Alignment Procedure for Three-Hole and Static Pressure Probe Mea- surements}

Although an effort was made to arrange that the two probes obtained data at the same pitchwise location during the experiment, the measurements from the three-hole and static pressure probes are compared during the data reduction routine to verify alignment. This eliminates any error that may have been introduced through sources such as backlash in the traverse gear. Static pressure measurements from the three-hole probe data reduction are compared with the measurements from the static pressure probe to align the two sets of data in the pitchwise direction. For supersonic flow, the two measurements of static pressures will differ by an amount equal to the static pressure rise across the probe bow shock. However, large gradients in static pressure, which will occur at the same pitchwise locations for the two sets of measurements, can be used as the basis for the probe alignment.

Misalignment of the probes can lead to errors in the loss measurements. Figure 4.5 shows an exaggerated example of the influence of probe misalignment. The pitchwise variation of the static pressure and the local total pressure loss coefficient $C_{p o}$ are shown in Figures 4.5(a) and 4.5(b) for E2S50 at an inlet flow angle of $14^{\circ}$ and an outlet Mach number of 1.15. The data from the two probes are shown as measured and after being misaligned by about 0.3 pitch spacings, s. The local total pressure loss coefficient is defined as,

$$
C_{p o}=\frac{P_{o 2}-P_{o 1}}{P_{o 2}-P_{2}}
$$

The error introduced by the misalignment of the static pressure data is evident by the region of negative losses shown in Figure 4.5(b), In this case due to the misalignment, the static pressure in the region between 0.7 and $0.9 \mathrm{y} / \mathrm{s}$ is measured to be lower than it is in actuality. Consequently, the Mach number in this region will be calculated erroneously high and, due to the Rayleigh correction, the total pressure drop across the probe bow shock will be overestimated. Therefore, the total pressure reduced from the three-hole probe data will be corrected by too great a factor and the data reduction calculation results in a downstream total pressure greater than that of the upstream total pressure (i.e: negative losses). However 


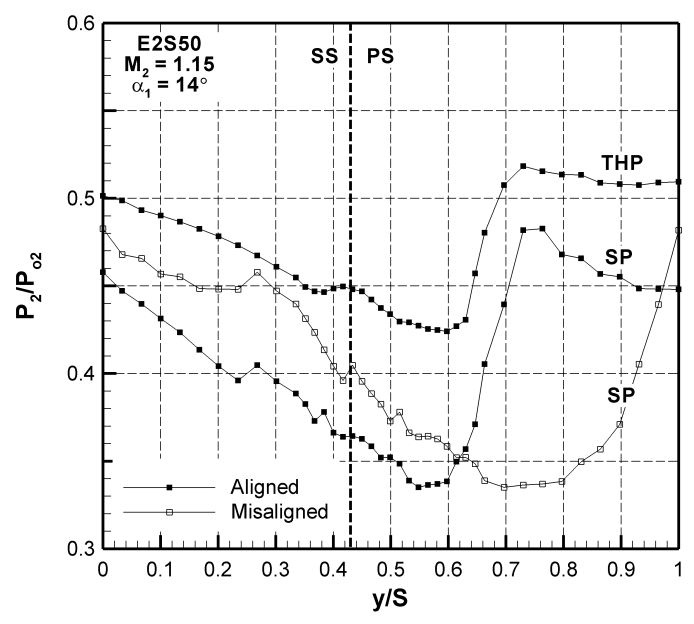

(a) Static Pressure Variation

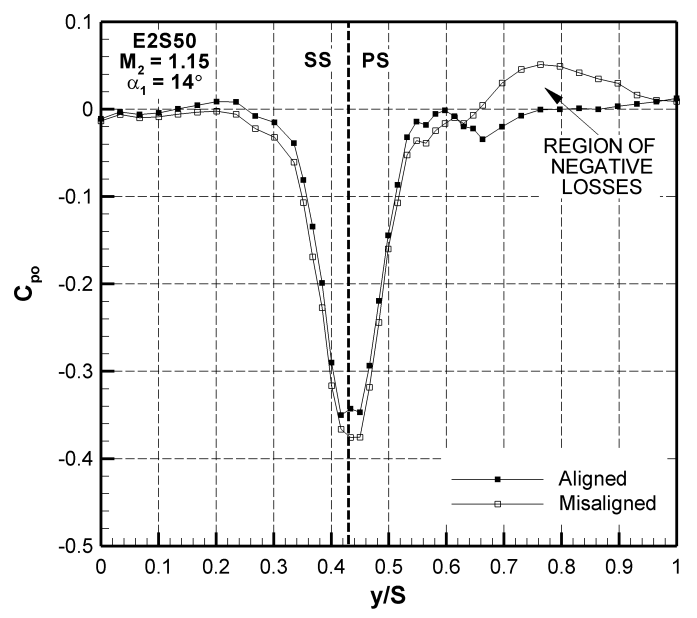

(b) Total Pressure Loss Coefficient Variation

Figure 4.5: Effect of Poorly Aligned Static Pressure Measurements

in general, the probe alignment resembles that shown by the filled symbols in Figure 4.5 .

\subsubsection{Averaging and Mixing Calculations of Non-Uniform Flow}

This section will describe the methods by which the nonuniform pitchwise measurements obtained from the data reduction procedures, presented previously, are averaged and mixedout. Area-averaging, mass-averaging and mixing-out calculations will be described. Cumpsty \& Horlock (2006) have shown that the quantitative difference between various averaging methods is small for the flows that are likely to occur in gas turbines. Mass-averaging does not account for the downstream losses which will occur due to the mixing of the wake and are therefore lower than the mixed-out losses. Area-averaged losses are estimated to be higher than mass-averaged since they are calculated with the assumption that each stream-tube carries the same mass flow rate. However in practice, the mass flow per unit area in the boundary layers and wake will be less than that of the free stream. Although the mixed-out losses will also include losses which occur downstream of the measuring plane, Jouini (2000) showed that for profile loss measurements, the additional losses are generally not large when compared with the losses obtained at the measuring plane. This is not true for secondary and tip-leakage losses. Mixed-out losses are those embedded in the loss correlations. Additionally, 
it is also necessary to calculate mixed-out losses to compare measurements from different experiments for which different traverse locations may have been used.

Figure 4.6 shows the nomenclature used for the averaging calculations. The subscripts $1,2 y$ and ${ }_{2}$ denote the inlet, measurement and mixed-out planes respectively. Additionally, area-averaged quantities will be denoted by the subscript ${ }_{A}$ and mass-averaged quantities by the subscript $\dot{m}$.

Following the nomenclature in Figure 4.6, the area-averaged value of an arbitrary flow property $\chi$ is,

$$
\left(\chi_{2}\right)_{A}=\frac{\int_{A} \chi_{2 y} d A}{\int_{A} d A}=\int_{0}^{1} \chi_{2 y} d(y / s)
$$

where $\chi$ refers to a downstream thermodynamic property (e.g.: pressure, temperature, Mach number, etc.), whether static or stagnation. However, area-averaging is typically limited to static pressure.

The mass-averaged value of $\chi$ is,

$$
\left(\chi_{2}\right)_{\dot{m}}=\frac{\int_{\dot{m}} \chi_{2 y} d \dot{m}}{\int_{\dot{m}} d \dot{m}}=\frac{\int_{0}^{1} \chi_{2 y}\left[P_{2 y} \frac{1}{\sqrt{T_{o 2 y}}} M_{2 y} \cos \alpha_{2 y}\left(1+\frac{\gamma_{2 y}-1}{2} M_{2 y}^{2}\right)^{\frac{1}{2}}\right] d(y / s)}{\int_{0}^{1}\left[P_{2 y} \frac{1}{\sqrt{T_{o 2 y}}} M_{2 y} \cos \alpha_{2 y}\left(1+\frac{\gamma_{2 y}-1}{2} M_{2 y}^{2}\right)^{\frac{1}{2}}\right] d(y / s)}
$$

where $\dot{m}$ is the mass flow rate.

To calculate the mixed-out flow properties, the method outlined by Amecke \& Šafařík (1995) is used in the current study. This method of averaging calculates the flow properties that would exist if the flow were permitted to mix to uniform conditions in a constant area channel with frictionless walls. For multi-stage turbomachines, the mixed-out losses may be considered to be pessimistic since the flow will not mix to uniform flow between blade rows. However, for cascade testing it provides a useful reference frame for loss comparison. Losses calculated from other types of averaging, such as area- or mass-averaged losses, will depend on the location of the measuring plane and do not account for the downstream mixing losses which are expected to occur. Area- and mass-averaged losses will tend to approach the mixed-out value as the measuring plane moves downstream. The Amecke \& Šafař́k method for mixing calculations does not account for the additional loss production that will take place in the endwall boundary layers downstream of the measurement plane. Although two-dimensional 


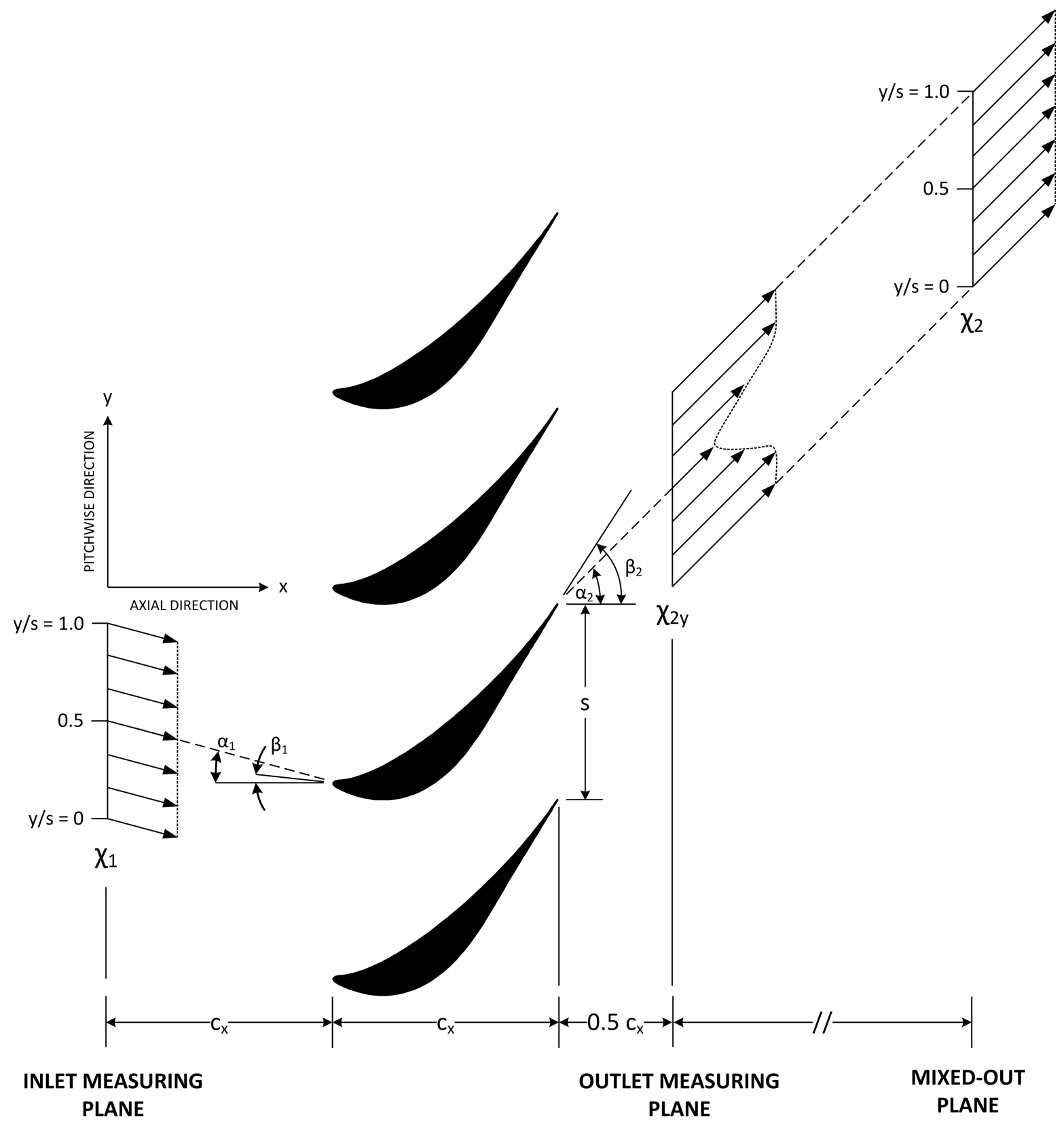

Figure 4.6: Nomenclature used for Mixing Calculation 
profile loss calculations will be unaffected by this simplification, mixing calculations of threedimensional losses will.

To solve for the mixed-out properties, the equations of conservation of mass, momentum and energy are solved for a control volume that extends from the measuring plane to a hypothetical downstream plane (the mixed-out plane in Figure 4.6), at which the flow properties are homogenous. A detailed derivation of the conservation equations for the flow field of a turbomachine can be found in Amecke \& Šafařík (1995). These are summarized in Appendix B

\subsection{Measurement Uncertainty}

The uncertainties on the measurements and calculations of the different quantities of interest in the HSWT were estimated by Corriveau (2005). The uncertainty analysis was repeated by Hall (2012) for lower Mach numbers. This section will provide a summary of the procedure used by Corriveau and Hall, as well as presents the uncertainties on the pressure measurement and the calculated parameters such as Mach number and total pressure loss coefficient.

The procedure followed by Corriveau and Hall was that which was specified in the AIAA Standard S-071A-1999 titled "Assessment of Experimental Uncertainty with Application to Wind Tunnel Testing".

Any derived parameter $F$ can be expressed as a function of fundamental quantities $\phi_{1}, \phi_{2}, \ldots, \phi_{N}$ as follows:

$$
F=F\left(\phi_{1}, \phi_{2}, \ldots, \phi_{N}\right)
$$

Furthermore, each fundamental quantity, $\phi_{i}$ can be expressed as,

$$
\phi_{i}=\hat{\phi}_{i}+\delta \phi_{i}
$$

where $\hat{\phi}_{i}$ is the true value and $\delta \phi_{i}$ the uncertainty. It follows that the contribution to the uncertainty of $\mathrm{F}$ due to the uncertainty in $\phi_{i}$ is,

$$
(\delta F)_{i}=\left(\frac{\partial F}{\partial \phi_{i}}\right) \delta \phi_{i}
$$




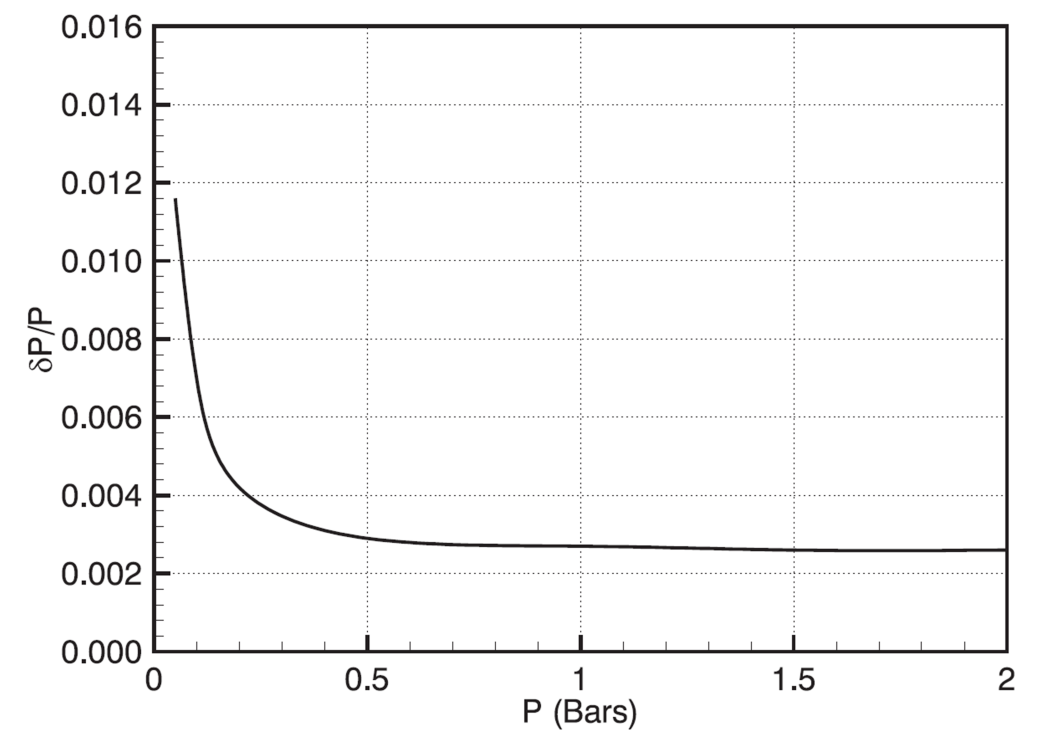

Figure 4.7: Variation of Uncertainty on Pressure Measurements (Corriveau, 2005)

If the values of the individual fundamental quantities are independent and normally distributed about the true value, the total uncertainty of $\mathrm{F}$ is given by,

$$
\delta F=\sqrt{\left(\frac{\partial F}{\partial \phi_{1}}\right)^{2} \delta \phi_{1}^{2}+\left(\frac{\partial F}{\partial \phi_{2}}\right)^{2} \delta \phi_{2}^{2}+\ldots+\left(\frac{\partial F}{\partial \phi_{N}}\right)^{2} \delta \phi_{N}^{2}}
$$

To provide an example, the uncertainty on the measurement of pressure will be considered. The pressure measurement from each pressure transducer is calculated using the calibration curve of the transducer as follows:

$$
P=m V+b
$$

where $\mathrm{P}$ is the pressure, $\mathrm{V}$ the measured transducer voltage, and $\mathrm{m}$ and $\mathrm{b}$ are the slope and zero pressure intercept of the transducer calibration curve. Therefore, the uncertainty of $\mathrm{P}$ can be expressed as,

$$
\delta P=\sqrt{\left(\frac{\partial P}{\partial m}\right)^{2} \delta m^{2}+\left(\frac{\partial P}{\partial V}\right)^{2} \delta V^{2}+\left(\frac{\partial P}{\partial b}\right)^{2} \delta b^{2}}
$$

By differentiating Equation 4.14 to obtain the partial derivatives, the uncertainty then becomes,

$$
\delta P=\sqrt{(V \delta m)^{2}+(m \delta V)^{2}+\delta b^{2}}
$$


Table 4.1: Summary of the Uncertainties Calculated by Corriveau (2005)

\begin{tabular}{lcc}
\hline & Range & Uncertainty Range \\
\hline \hline Pressure, $P$ & 0.5 to 1.5 bars & $\pm 0.3 \%$ \\
Inlet Flow Angle, $\alpha_{1}$ & $-30^{\circ}$ to $30^{\circ}$ & $\pm 1.0^{\circ}$ \\
Outlet Flow Angle, $\alpha_{2}$ & $\pm 10^{\circ}$ relative to probe axis & $\pm 0.5^{\circ}$ \\
Inlet Mach number, $M_{1}$ & $<0.6$ & \pm 0.008 \\
Outlet Mach number, $M_{2}$ & 0.8 to 1.3 & \pm 0.005 \\
Loss Coefficient, $Y$ & $M_{2, \text { is }}$ from 0.5 to 1.25 & \pm 0.020 to \pm 0.007 \\
Reynolds Number, $R e_{c}$ & $M_{2, i s}$ from 0.5 to 1.25 & \pm 5500 to \pm 97000 \\
\hline
\end{tabular}

The uncertainty on the measurements of the transducer voltages was obtained by Jeffries (2000). At a sampling frequency of $2 \mathrm{kHz}$, Jeffries found with a $95 \%$ confidence limit that the uncertainty in the mean voltage was about $\pm 0.25 \%$ of the absolute value after a sampling time of 0.1 seconds. Consequently, the uncertainty in voltage, and hence in pressure, is dependent on the pressure.

The variation of the uncertainty on the pressure measurement is shown in Figure 4.7. For the range of operating conditions in the current study, the pressure did not drop below about $60000 \mathrm{kPa}$ (0.6 bar). Therefore, the uncertainty in pressure is roughly constant at about $\pm 0.3 \%$ of the absolute pressure measured.

Following the same procedure, Corriveau calculated the uncertainties of other flow parameters. These are summarized in Table 4.1 .

\subsection{Flow Quality}

\subsubsection{Introduction}

Linear cascade testing offers a valuable tool for gaining insight into the flow physics of turbine blade passages. However, for the results of the cascade to be useful, great care must be exercised to ensure that the measurements are not dependent upon the wind tunnel in which they were obtained. To achieve this, the blade for which measurements are obtained must 
behave similarly aerodynamically to a blade that is part of a cascade with an infinite number of blades. For midspan measurements, the cascade must also have a significant region of uniform flow in the spanwise direction about the midspan of the inlet, uniform static pressure in the pitchwise direction at the inlet, and periodic flow behavior downstream of the cascade. The influence of poor flow quality, particularly non-uniform inlet flow, was investigated by Corriveau \& Sjolander (2002). This section shows the flow quality for the three cascades measured in the current study. The work of Corriveau (2005), who used the same wind tunnel as the current study, will serve as a guide when considering the flow quality.

\subsubsection{Inlet Endwall Boundary Layer}

As shown in Figure 3.3, before reaching the cascade the inlet flow travels a distance of about $2 \mathrm{~m}$ in the test section. Consequently, at the cascade inlet the boundary layer that develops on the endwalls of the test section has a thickness which is a significant fraction of the blade span. Therefore, the inlet flow was traversed in the spanwise direction about $40 \mathrm{~mm}$ upstream of the centre blade. For two-dimensional cascade studies, it is necessary that the flow be uniform over a significant region around the midspan. The HSWT has two boundary layer bleed slots upstream of the cascade which can be outfitted with porous plates to blow off air from the test section and thus reduce the thickness of the inlet boundary layer. However, as will be seen, these were not necessary for the current study.

The traverse measurements were obtained using the boundary layer probes described in Section 3.4.3. A total of 60 measurements were obtained over the span from roughly $12 \%$ to $88 \%$ of the span at three exit Mach numbers for the first cascade tested, E2S50.

Figure 4.8 shows the spanwise variation of the inlet Mach number for E2S50 at three outlet isentropic Mach numbers: 0.79. 0.98, and 1.20. There is very little difference between the three traverses suggesting that the cascade is choked for all three operating points. Although the boundary layer was not traversed in its entirety, the edge of the boundary layer can be

observed within the data. The boundary layer thickness $\delta_{99 \%}$ is estimated to be between 10 and $15 \mathrm{~mm}$ (roughly $20 \%$ of the span) for all Mach numbers and appears to be symmetrical about the midspan. This is similar to the results obtained by Corriveau (2005). Corriveau 


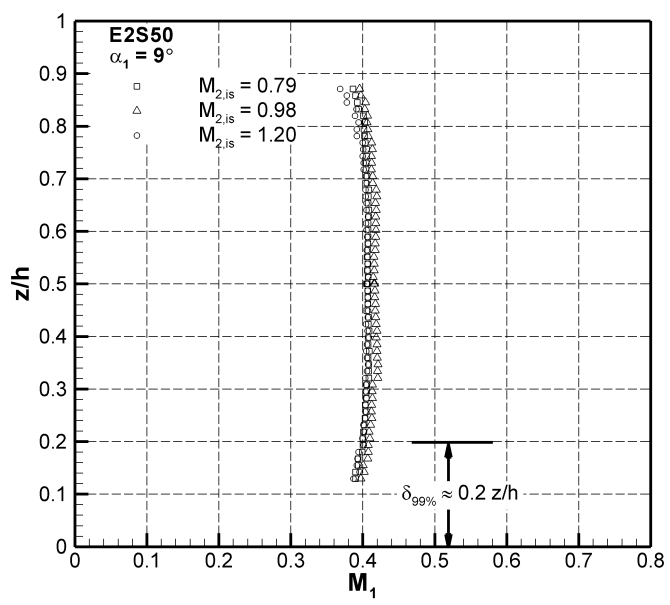

Figure 4.8: Inlet Spanwise Variation of Mach Number for E2S50 at $\alpha_{1}=9^{\circ}$

obtained detailed measurements of the inlet boundary layer at several exit Mach numbers between 0.5 and 1.16 and showed that the boundary layer parameters where similar between choked and unchoked flow conditions. For both conditions, Corriveau calculated the boundary layer shape factor $H$ to be about 1.4, which is typical of a zero pressure-gradient turbulent boundary layer. The shape factor is defined as,

$$
H=\frac{\delta^{*}}{\theta}
$$

where $\delta^{*}$ and $\theta$ are the displacement and momentum thicknesses, which are defined for compressible boundary layers as,

$$
\delta^{*}=\int_{0}^{\infty}\left(1-\frac{\rho}{\rho_{e}} \frac{u}{U_{e}}\right) d z
$$

and,

$$
\theta=\int_{0}^{\infty} \frac{\rho}{\rho_{e}} \frac{u}{U_{e}}\left(1-\frac{u}{U_{e}}\right) d z
$$

where $\rho_{e}$ and $U_{e}$ are the fluid density and velocity at the boundary layer edge.

Although the cascade shows a significant region of uniform inlet flow at the midspan, Corriveau showed that for a cascade with similar inlet conditions, the outlet two-dimensionality 
of the flow suffered at positive values of incidence. Corriveau attributed the decline in twodimensionality to the increased loading and decreased passage acceleration at positive incidences. The outlet two-dimensionality is partially dependent on the secondary flow structures, which originate from the upstream endwall boundary layer. The strength of the secondary vortex increases at positive incidences due to the increased cross-passage pressure gradient. On the other hand, high amounts of acceleration tend to limit the spanwise dimension of the vortex. Thus, at positive incidences, as the acceleration is reduced, the extent of the region of two-dimensional flow at the midspan will also be reduced.

Outlet two-dimensionality can be evaluated by obtaining loss measurements at several span wise locations downstream of the cascade. However, no such measurements were obtained for the current study. The majority of the results discussed in this study were obtained near the design incidence. Corriveau showed that at design incidence, outlet two-dimensionality was acceptable. Furthermore, the current cascades have higher channel acceleration than the cascades measured by Corriveau with similar aspect ratios. Therefore, it is likely that the outlet two-dimensionality is similar to, if not slightly better than that observed by Corriveau.

\subsubsection{Inlet Pitchwise Flow Uniformity}

The inlet static pressure is measured at the endwall at a distance of $1.0 c_{x}$ upstream of the leading edge to verify uniformity in the pitchwise directions. The inlet flow is controlled through the alignment and spacing of the adjustable side walls. The appropriate spacing of the side walls can be calculated for a cascade of $\mathrm{N}$ blades, with a pitch spacing $s$ and inlet flow angle $\alpha_{1}$ as follows. The spacing between bounding streamlines for the stream tube entering one passage is given by,

$$
w=s \cos \alpha_{1}
$$

Therefore, the total width of the cascade with $\mathrm{N}+1$ passages is

$$
W=(N+1) \times w
$$

This method ignores blockage due to the boundary layers that develop on the inlet sidewalls. The boundary layers on the sidewalls are expected to be thin since the flow over much of the 


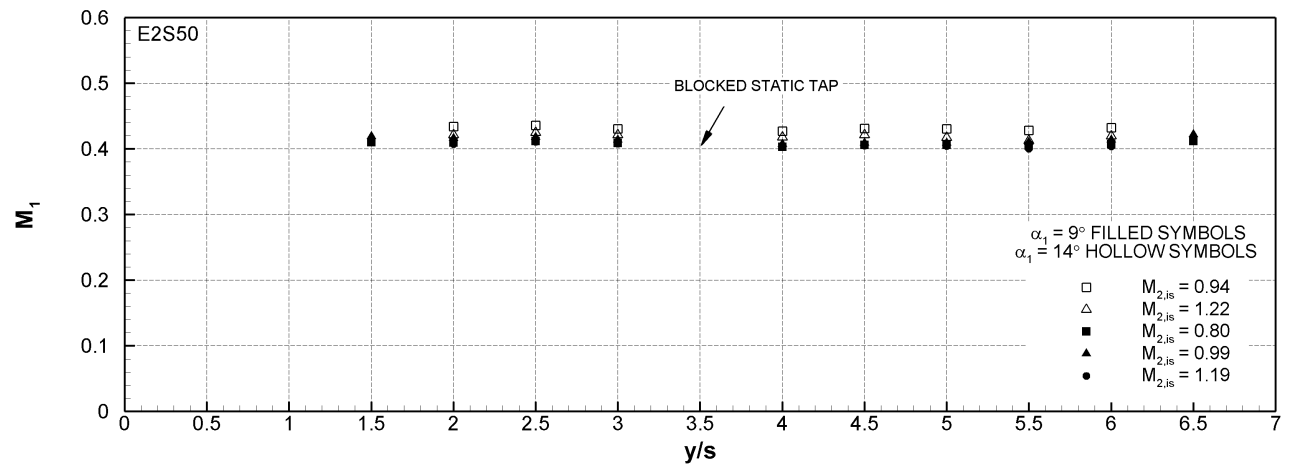

Figure 4.9: Inlet Pitchwise Flow Uniformity for E2S50 Near Design Incidence

sidewall length is highly accelerated. Although thought to be small, the sidewall boundary layers will have an effect on the flow in the two outermost blade passages. There is no provision to adjust the flow through these outermost passages.

The inlet flow uniformity is measured at each incidence to ensure that the mass flow rates through all passages are equal. For early measurements of E2S50, inlet pitchwise variations of Mach number, calculated from the centreline total pressure and the endwall static pressure, were obtained at several values of outlet Mach number and are shown in Figure 4.9. As expected for choked flow, there is no influence of exit Mach number on inlet flow uniformity. Therefore, subsequent uniformity measurements were obtained at only one value of exit Mach number. The good inlet uniformity shown in Figure 4.9 suggests that the inlet side walls are correctly aligned and spaced properly.

The influences of non-uniform inlet flow are discussed by Corriveau (2005). When the inlet side walls are misaligned, a static pressure gradient is observed, suggesting curvature in the inlet streamlines. Consequently, the inlet angle would likely vary from passage to passage. This would lead to error in the loss measurements, particularly at large values of incidence at which the cascade becomes sensitive to changes in the inlet angle.

\subsubsection{Outlet Flow Periodicity}

In an annular turbine with uniform inlet flow, the outlet flow will naturally be periodic. In linear turbine cascades, perfect periodicity is only possible for a cascade with an infinite 
number of blades. For real cascades with a finite number of blades, outlet flow periodicity is only approximated over several blade passages in the centre of the cascade. As was shown in Chapter 3, the outlet flow from the cascade discharges into a channel with considerably larger area than the cascade. Consequently, the exit flow develops as a free jet. Control surfaces are not used to adjust the outlet flow. For supersonic outlet flow, the use of control surfaces such as tailboards can lead to errors in wake measurements caused by reflected shocks.

There are several suggested methods by which the outlet flow periodicity can be measured. For example, Starken \& Lichtfuss (1975) suggest the same procedure used for measuring inlet flow uniformity: that is, measuring static pressure at the endwall across the cascade. Other authors have suggested comparing blade loading distributions for several blades in the cascade. However, for the current study, both endwall static pressure measurments as well as midspan wake measurements were obtained at $0.5 c_{x}$ downstream of the trailing edge for the five center passages. To evaluate the periodicity, the pitchwise variation of the total pressure loss coefficient and the outlet flow angle are measured for each passage and the mixed out values of these parameters are compared.

Figures 4.10 and 4.11 show the periodicity of losses and outlet flow angle for the three Siemens cascades for which the midspan flows were measured. Periodicity measurements were obtained at an exit Mach number near 1.0 for E2S50 and E2S90, and at about 1.1 for E1S50. Although periodicity measurements are obtained for each value of inlet angle, the results are shown only for the two values of inlet angle that are estimated to be closest to the design inlet angle. These are representative of the results obtained at all values on inlet flow angle. Discussion of the method by which the design inlet angle was estimated is given in Chapter 5. The blade numbers indicated on Figures 4.10 and 4.11 correspond to Figure 4.1 .

Static pressure data measured for the periodicity assessment were reduced from three-hole probe data, rather than from data obtained with the static probe. Consequently, it was not possible to use the Rayleigh pitot formula (Equation 4.6) to correct the total pressure. Therefore, the values of losses obtained from the measurements include those losses generated in the bow shock that forms due to the presence of the probe. Hence, the losses appear high. Since the purpose of these measurements was simply to gauge passage-to-passage periodicity, 


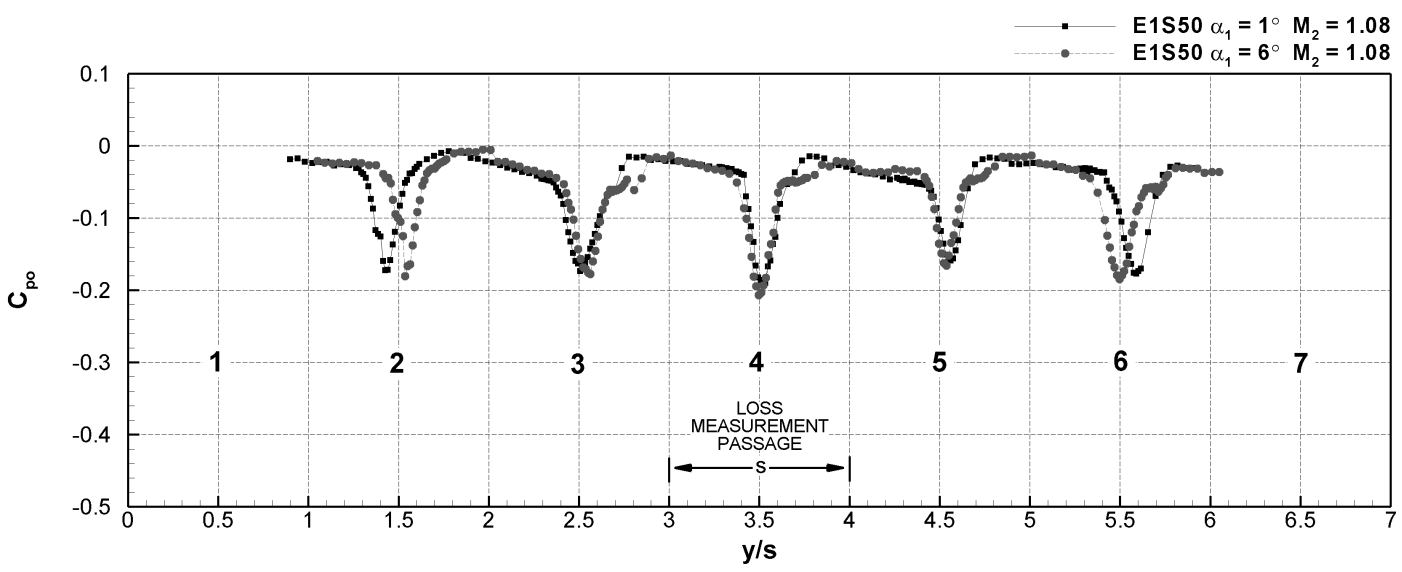

(a) E1S50

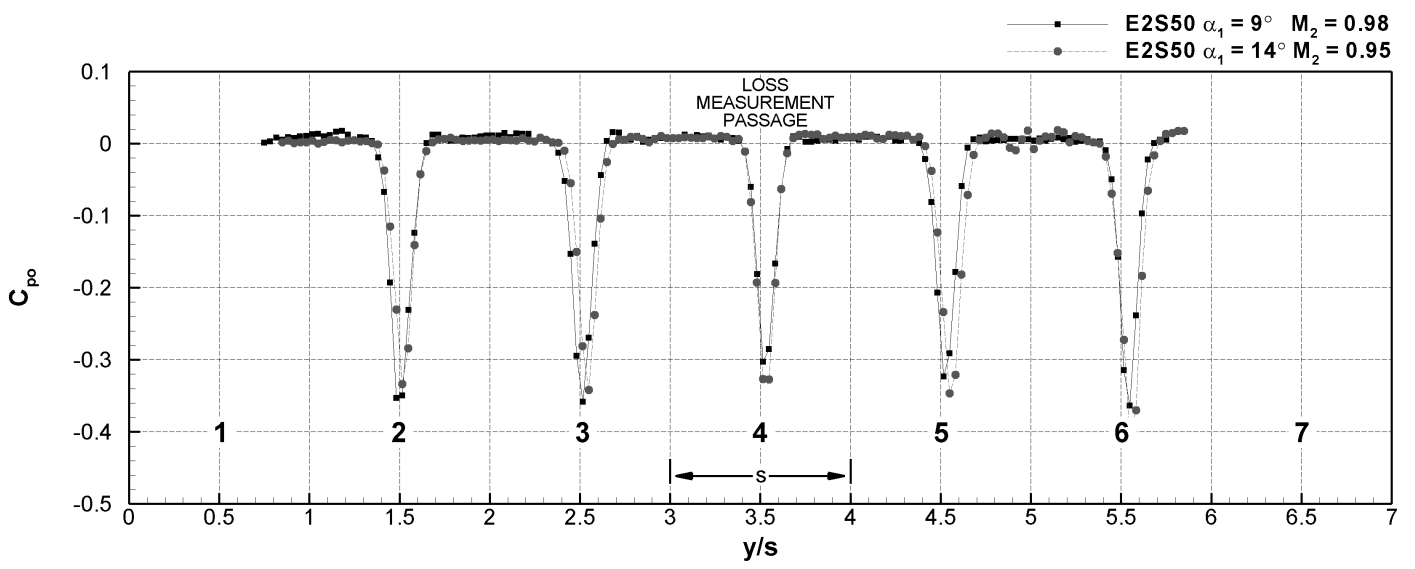

(b) E2S50

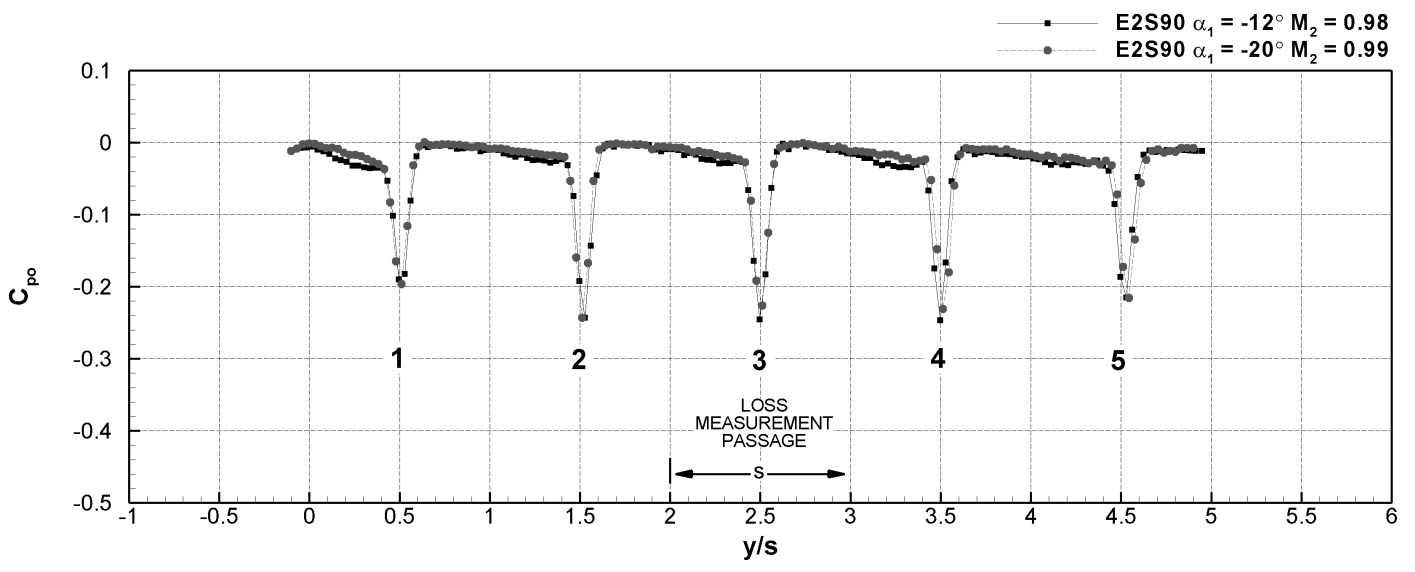

(c) E2S90

Figure 4.10: Total Pressure Loss Periodicity 


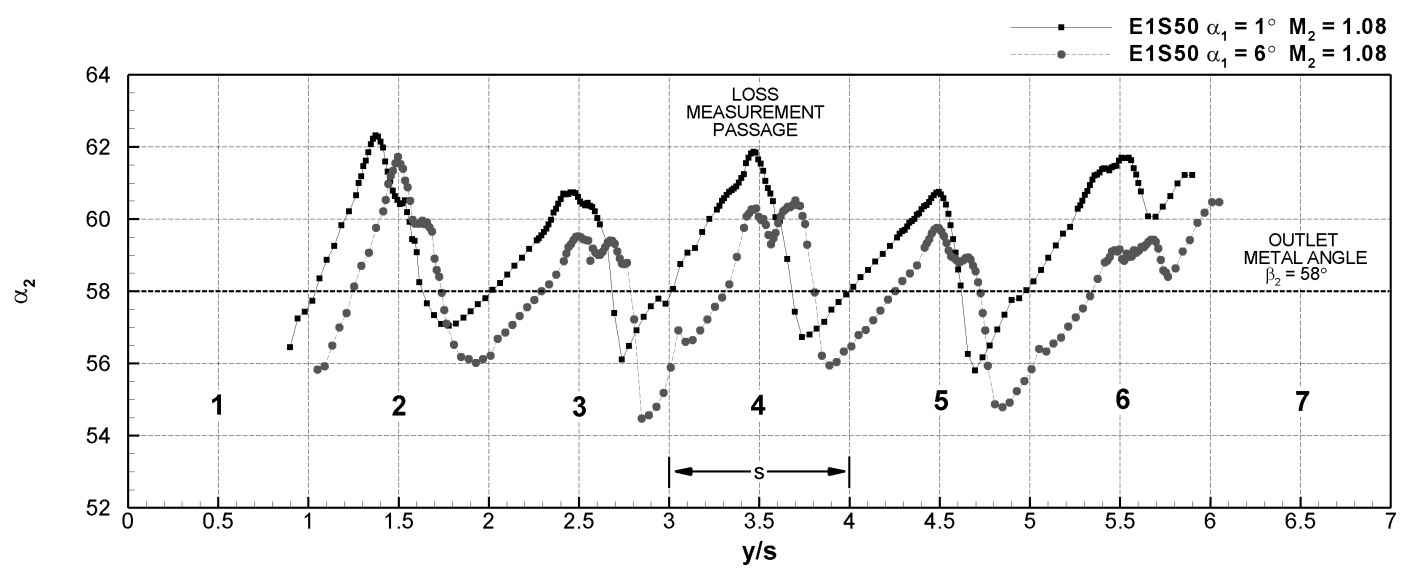

(a) E1S50

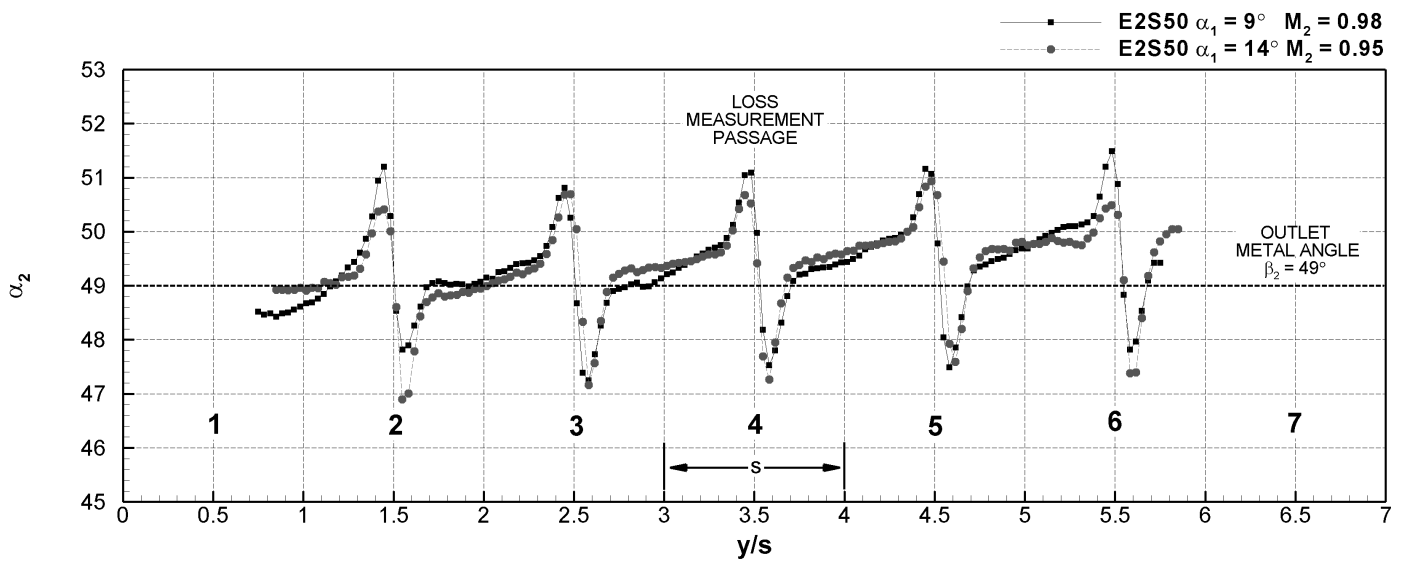

(b) E2S50

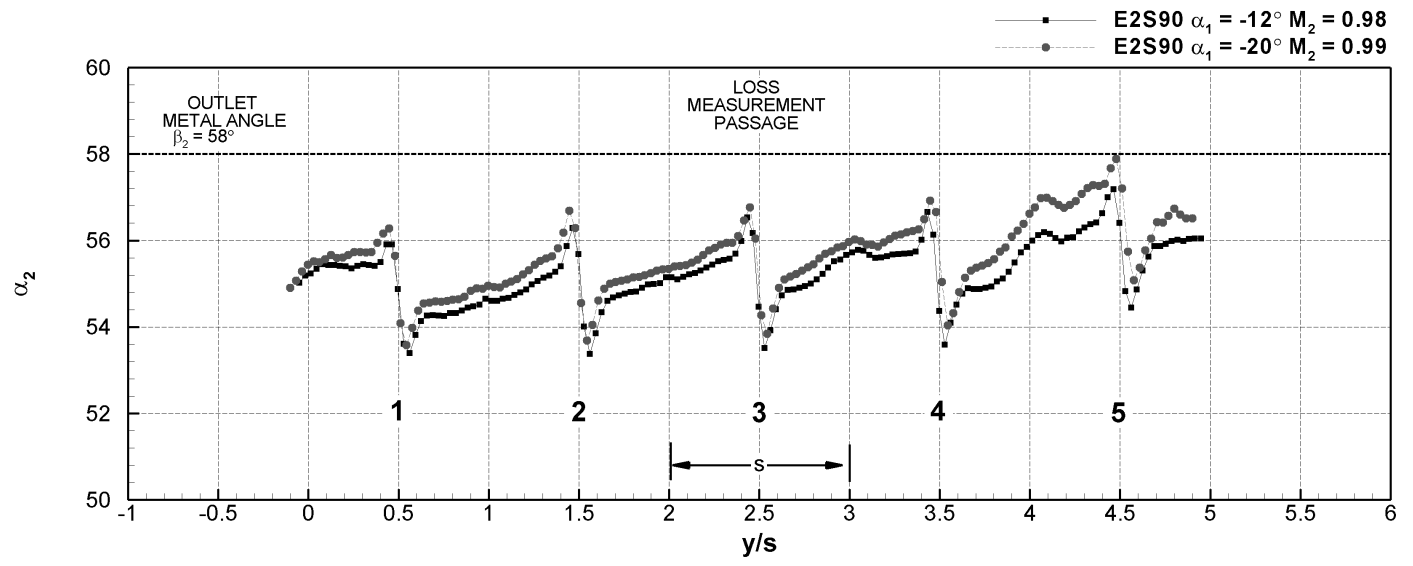

(c) E2S90

Figure 4.11: Outlet Flow Angle Periodicity 
and the effect of the probe bow shock is roughly consistent across the cascade, the measurements shown in Figure 4.10 are adequate. Furthermore, due to the coarse measurement spacing, error in the mixed-out losses is expected to be non-negligible. Therefore, periodicity measurements should be interpreted qualitatively, as well as quantitatively.

E1S50 shows the most blade-to-blade variation in total pressure loss and flow angle. The Mach number at which this cascade was tested is higher than for the other two cascades. At supersonic Mach numbers the shock system in the flow passage may not be entirely stable, particularly since the operating point is known to fluctuate. Furthermore, the slight variations in geometry, such as differences in throat area from passage to passage, could lead to greater variation in losses at higher Mach numbers.

The blade-to-blade variation of total pressure loss and flow angle show good periodicity over the centre five passages for E2S50. However, E2S90 shows decreased wake depth for blades 1 and 5. As mentioned in Chapter 3, the tip cascade had fewer blades than the two midspan cascades. Blades 1 and 5 of the tip section cascade are two outermost blades. Losses for these blade are effected by the inlet side wall boundary layers.

The pitchwise variations were mixed-out using the method of Amecke \& Šafařík (1995) described in Section 4.4.4. The mixed out values of Mach number $M_{2}$, total pressure loss coefficient Y, and flow angle $\alpha_{2}$ are summarized in Table 4.2. The average values and standard deviation are shown as well.

The mixed out values of Mach number show good periodicity, particulary at lower Mach numbers. The standard deviation for the subsonic Mach numbers are similar to those measured by Corriveau (2005) in the same facility.

The absolute variation in total pressure loss coefficient $Y$ is similar to that measured by Corriveau. However, since the losses for the Siemens cascades are lower than for the cascades measured by Corriveau, the relative variations in $Y$ are larger. Of the Siemens cascades, E2S50 shows the lowest variation in losses. This could be caused by the inconsistency in the chemical polishing described in Chapter 3 .

The outlet flow angle of E1S50 shows significant variation in outlet flow angle. As mentioned, this could be due in part to the shock system that develops at supersonic conditions. 
Table 4.2: Mixed-Out Periodicity

\begin{tabular}{|c|c|c|c|c|c|c|}
\hline Blade $N^{o}$ & $M_{2}$ & $Y$ & $\alpha_{2}$ & $M_{2}$ & $Y$ & $\alpha_{2}$ \\
\hline E1S50 & \multicolumn{3}{|c|}{$\alpha_{1}=1^{\circ}$} & \multicolumn{3}{|c|}{$\alpha_{1}=6^{\circ}$} \\
\hline 2 & 1.05 & 0.0442 & $59.0^{\circ}$ & 1.05 & 0.0407 & $58.1^{\circ}$ \\
\hline 3 & 1.09 & 0.0618 & $58.7^{\circ}$ & 1.09 & 0.0617 & $57.6^{\circ}$ \\
\hline 4 & 1.07 & 0.0533 & $59.1^{\circ}$ & 1.07 & 0.0602 & $58.2^{\circ}$ \\
\hline 5 & 1.08 & 0.0568 & $58.5^{\circ}$ & 1.08 & 0.0521 & $57.4^{\circ}$ \\
\hline 6 & 1.09 & 0.0562 & $60.1^{\circ}$ & 1.11 & 0.0619 & $58.5^{\circ}$ \\
\hline Mean & 1.08 & 0.0545 & $59.1^{\circ}$ & 1.08 & 0.0553 & $58.0^{\circ}$ \\
\hline Std. Deviation, $\sigma$ & 0.0164 & 0.0058 & $0.57^{\circ}$ & 0.0203 & 0.0081 & $0.40^{\circ}$ \\
\hline E2S50 & \multicolumn{3}{|c|}{$\alpha_{1}=9^{\circ}$} & \multicolumn{3}{|c|}{$\alpha_{1}=14^{\circ}$} \\
\hline 2 & 0.98 & 0.0283 & $49.2^{\circ}$ & 0.95 & 0.0295 & $49.0^{\circ}$ \\
\hline 3 & 0.97 & 0.0281 & $49.2^{\circ}$ & 0.95 & 0.0282 & $49.3^{\circ}$ \\
\hline 4 & 0.98 & 0.0229 & $49.4^{\circ}$ & 0.95 & 0.0255 & $49.5^{\circ}$ \\
\hline 5 & 0.98 & 0.0266 & $49.6^{\circ}$ & 0.95 & 0.0283 & $49.7^{\circ}$ \\
\hline 6 & 0.98 & 0.0292 & $49.8^{\circ}$ & 0.95 & 0.0354 & $49.8^{\circ}$ \\
\hline Mean & 0.98 & 0.0270 & $49.4^{\circ}$ & 0.95 & 0.0294 & $49.5^{\circ}$ \\
\hline Std. Deviation, $\sigma$ & 0.0017 & 0.0022 & $0.25^{\circ}$ & 0.0012 & 0.0033 & $0.27^{\circ}$ \\
\hline E2S90 & \multicolumn{3}{|c|}{$\alpha_{1}=-12^{\circ}$} & \multicolumn{3}{|c|}{$\alpha_{1}=-20^{\circ}$} \\
\hline 1 & 0.98 & 0.0310 & $54.9^{\circ}$ & 0.98 & 0.0253 & $55.2^{\circ}$ \\
\hline 2 & 0.98 & 0.0310 & $54.9^{\circ}$ & 0.98 & 0.0264 & $55.2^{\circ}$ \\
\hline 3 & 0.98 & 0.0322 & $55.2^{\circ}$ & 0.98 & 0.0267 & $55.5^{\circ}$ \\
\hline 4 & 0.98 & 0.0379 & $55.4^{\circ}$ & 0.99 & 0.0309 & $55.8^{\circ}$ \\
\hline 5 & 0.99 & 0.0370 & $56.0^{\circ}$ & 1.00 & 0.0350 & $56.7^{\circ}$ \\
\hline Mean & 0.98 & 0.0338 & $55.3^{\circ}$ & 0.99 & 0.0288 & $55.7^{\circ}$ \\
\hline Std. Deviation, $\sigma$ & 0.0027 & 0.0030 & $0.42^{\circ}$ & 0.0073 & 0.0036 & $0.56^{\circ}$ \\
\hline
\end{tabular}


E2S90 also shows some variation in flow angle. Since the tip section periodicity measurements include the two outermost passages, the blade-to-blade variation is expected to be higher than for the other two cascades. Figure 4.11(c) shows that the fifth blade passage for E2S90 has significantly higher values of exit flow angle. This is likely an indication that the outlet flow from this passage is expanding into the larger area downstream of the trailing edge. Similar to the losses, the periodicity of the outlet flow angle is best for E2S50. 


\section{Chapter 5}

\section{Experimental Results}

\subsection{Introduction}

Measurements of midspan losses and outlet flow angles were obtained for three of the Siemens cascades: the two midspan geometries, E1S50 and E2S50, and the tip section E2S90. This chapter examines the influence of the operating conditions on the performance of the cascades. The discussion will be focused on the influence of outlet Mach number $M_{2}$ on the total pressure loss coefficient $Y$, and the outlet flow angle $\alpha_{2}$ of the cascades near design incidence; however some discussion of the effects of incidence is also included. The off-design incidence results are used to estimate the design incidence.

Cascade performance at design incidence is discussed in detail. The results are compared with predictions from the loss correlation system of Kacker \& Okapuu (1982) (KO) for design incidence. The loss predictions for a fourth cascade, for which no experimental data exists, are also included. This fourth cascade, the tip section E1S90, has a large negative inlet angle. Shortcomings of the KO loss system are noted when predicting the losses of turbine geometries with large negative inlet angles.

Reynolds number effects are discussed. An alternative to the Kacker \& Okapuu correction factor for Reynolds number, due to Aungier (2006), is described. The two Reynolds corrections are compared with the data for E2S50. This cascade is not hydraulically smooth. Therefore, the influence of surface roughness on loss prediction could be examined.

Finally, several recommendations are made for the improvement of the Kacker \& Okapuu 
loss system. Included in the recommendations are suggested future studies which could further the understanding of loss behaviour of turbine cascades, specifically at large negative inlet angles.

\subsection{Test Matrix}

Each cascade was tested at a range of outlet Mach numbers $M_{2}$ and inlet flow angles $\alpha_{1}$. Reynolds number based on outlet flow conditions and blade chord $R e_{c}$ varies as well, however not independently of Mach number. The wind tunnel used for this study is equipped with an ejector-diffusor system, described by Corriveau (2005), which gives the wind tunnel the capability to vary Mach and Reynolds number independently. The ejector-diffusor system operates using the same air supply as the main test section. Because the air supply is limited for each run, achieving suitable run times for high Mach number tests can be difficult. Even without the ejector-diffusor system, up to four runs were required to measure the downstream wake across a single blade pitch at every operating point. Therefore, the ejector-diffusor system was not used for this study.

Table 5.1 shows the complete test matrix. For reference, the inlet metal angle $\beta_{1}$ of each cascade is included. Table 5.1 also indicates that blade loadings were measured only for E2S50. As mentioned in Chapter 3, this was the only cascade thick enough to be instrumented with static taps.

Originally, the intention was to test the cascades at four exit Mach numbers between 0.9 and 1.2. However, initial tests of E2S50 at $\alpha_{1}=9^{\circ}$ yielded mixed-out Mach numbers below the target values. Tunnel operating settings, such as blowing pressure and control system gains, were modified to reach the desired outcome. Subsequent tests for E2S50 were only conducted at the new operating points. Consequently, losses were measured at eight exit Mach numbers at an inlet angle of $9^{\circ}$ and four at all other inlet angles. Blade loading measurements at $9^{\circ}$, however, were obtained only at the original four Mach numbers.

The range of Mach numbers tested for E1S50 was broadened because the total cascade throat area allowed for increased run times at higher Mach numbers. The total cascade throat 
Table 5.1: Wind Tunnel Operating Conditions

\begin{tabular}{|c|c|c|c|c|}
\hline & $\alpha_{1}$ & $M_{2}$ & $R e_{c} \times 10^{-3}$ & Loadings \\
\hline \multirow{4}{*}{$\begin{array}{l}\text { E1S50 } \\
\beta_{1} \approx 6^{\circ}\end{array}$} & $1^{\circ}$ & $\begin{array}{c}0.92,0.97,1.02,1.03,1.05 \\
1.12,1.21,1.23,1.37,1.41\end{array}$ & $852-1400$ & - \\
\hline & $6^{\circ}$ & $\begin{array}{c}0.87,0.95,1.00,1.02,1.06 \\
1.11,1.20,1.30,1.33,1.41\end{array}$ & $817-1250$ & - \\
\hline & $15^{\circ}$ & $\begin{array}{c}0.91,0.96,1.03,1.06,1.07 \\
1.12,1.23,1.26,1.34,1.35\end{array}$ & $817-1280$ & - \\
\hline & $25^{\circ}$ & $\begin{array}{c}0.87,0.93,1.00,1.06,1.07 \\
1.11,1.22,1.25,1.36\end{array}$ & $788-1300$ & - \\
\hline \multirow{6}{*}{$\begin{array}{c}\mathbf{E 2 S 5 0} \\
\beta_{1} \approx 18^{\circ}\end{array}$} & $0^{\circ}$ & $0.85,0.94,1.03,1.15$ & $854-1330$ & $\checkmark$ \\
\hline & & $0.91,1.00,1.08,1.16$ & & - \\
\hline & $14^{\circ}$ & $0.92,1.02,1.10,1.18$ & $914-1370$ & $\checkmark$ \\
\hline & $19^{\circ}$ & $0.94,1.02,1.11,1.16$ & $954-1400$ & $\checkmark$ \\
\hline & $24^{\circ}$ & $0.93,1.01,1.09,1.19$ & $940-1360$ & $\checkmark$ \\
\hline & $29^{\circ}$ & $0.92,1.00,1.09,1.17$ & $910-1380$ & $\checkmark$ \\
\hline \multirow{3}{*}{$\begin{array}{c}\text { E2S90 } \\
\beta_{1} \approx-13^{\circ}\end{array}$} & $-30^{\circ}$ & $0.86,0.96,1.09,1.18$ & $692-975$ & - \\
\hline & $-20^{\circ}$ & $0.87,0.97,1.09,1.16$ & $703-1030$ & - \\
\hline & $-12^{\circ}$ & $0.88,0.99,1.10,1.17$ & $719-982$ & - \\
\hline
\end{tabular}

area for E1S50 is 20\% smaller than that of E2S50 and 11.5\% smaller than E2S90. Because of the ability to reach higher Mach numbers, E1S50 was the best candidate for a more detailed investigation on the effects of Mach number. Therefore, additional test points were added near $M_{2}=1.0$ as well.

Despite having detailed knowledge of the profiles for the cascades, certain design parameters were unclear, including the intended design incidence. Consequently, the cascades were not necessarily tested at their design incidence. As a first approximation, the design incidence was assumed to be at or near the inlet metal angle. Each cascade, with the exception of E2S50, was first tested at the assumed design inlet angle. E2S50 was first tested at $\alpha_{1}=9^{\circ}$ based upon suggestions from Siemens. Measurements at off-design incidence were obtained in 
$10^{\circ}$ intervals until a loss bucket was observed. Additional tests at $5^{\circ}$ intervals were selectively conducted for E1S50 and E2S50 to help identify a minimum loss incidence. A minimum loss incidence for E2S90 was not found within the range of measurements which were limited to a minimum inlet angle of $-30^{\circ}$ by wind tunnel capabilities.

\subsection{Blade Loadings for E2S50}

Blade loading measurements were obtained only for E2S50. As noted, this was the only cascade with sufficient thickness to accommodate the internal channels required for instrumenting the blades with surface static taps at midspan. Even for E2S50, the static taps were limited to the thickest parts of the airfoil, between 0.05 and $0.80 x / c_{x}$. Due to this limitation, there is no provision for measuring base pressure. This is unfortunate since base pressure has a significant influence on profile losses at transonic conditions in particular.

Base pressure $P_{b}$ is the local static pressure on the trailing edge in a region called the base region. Denton \& Xu (1990) describe the base region as a roughly triangular region of almost uniform pressure bounded by the separated shear layers from the pressure and suction surfaces. Using a control volume analysis for incompressible flow, Denton (1993) has shown that trailing edge losses are influenced by the base pressure, momentum thickness $\theta$ and displacement thickness $\delta^{*}$ of the blade-surface boundary layers as follows:

$$
Y_{t e}=\frac{\Delta P_{0}}{\frac{1}{2} \rho V_{2}^{2}}=-\frac{C_{P_{b}} t}{o}+\frac{2 \theta}{o}+\left(\frac{\delta^{*}+t}{o}\right)^{2}
$$

where the base pressure coefficient $C_{P_{b}}$ is

$$
C_{P_{b}}=\frac{P_{b}-P_{2}}{\frac{1}{2} \rho V_{2}^{2}}
$$

$o$ is the throat width and $t$ the blade thickness at the trailing edge.

Denton suggests that base pressure has an effect on losses generated in the downstream mixing of the pressure and suction side boundary layers. Furthermore, base pressure influences the shock system that forms by the sudden expansion at the trailing edge and also effects the pressure drag on the airfoil. The influence of base pressure on losses varies as the trailing edge blockage $t / o$, as evident by the first term in Equation 5.1. Fortunately, the Siemens cascades 


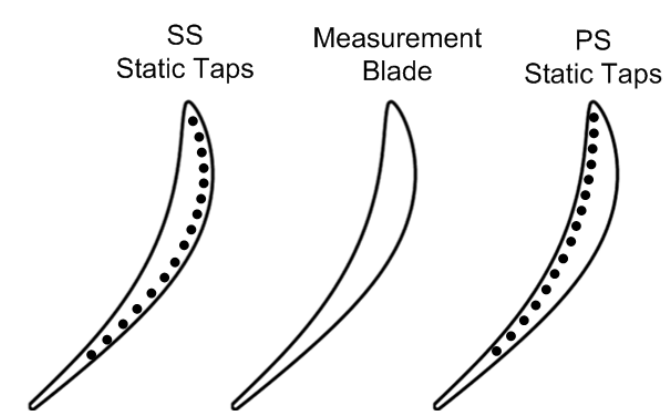

Figure 5.1: Blade Loading Static Tap Arrangement

all have thin trailing edges with trailing edge blockage less than $5 \%$. Hence, the influence of base pressure on losses is probably small.

Blade loadings for E2S50 were measured at the operating conditions shown in Table 5.1 . Static pressure measurements were obtained on the pressure side (PS) and the suction side (SS) using 16 static taps spaced by $0.05 x / c_{x}$ for each surface. The static taps are normal to the blade surface and $0.4 \mathrm{~mm}$ in diameter. The presence of static taps on the surface of the blades can affect the flow behaviour. Thus, to avoid measurement error, the blade for which losses were measured is not instrumented. Instead, the two adjacent blades are fitted with static taps in such a way that the taps share a flow passage with the loss measurement blade, as shown in Figure 5.1

The distributions of the isentropic Mach number $\left(M_{s, i s}\right)$ on the blade surface for E2S50 at all operating conditions are shown in Figure $5.2, M_{s, i s}$ is based on the local static pressure measured at the blade surface and the total pressure measured on the test-section centreline upstream of the cascade.

Figure 5.2(a) shows the distribution of $M_{s, i s}$ for E2S50 at an inlet flow angle $\alpha_{1}=9^{\circ}$. At this incidence, the flow along the suction surface accelerates from the leading edge to $x / c_{x}=0.55$, followed by a small region of constant surface Mach number; this is true at all exit Mach numbers tested. For smooth airfoils with comparable Reynolds numbers, the fact that the flow is accelerating would suggest that the flow over the forward part of the suction surface could be laminar. However, the surface roughness of E2S50 may act to promote the 


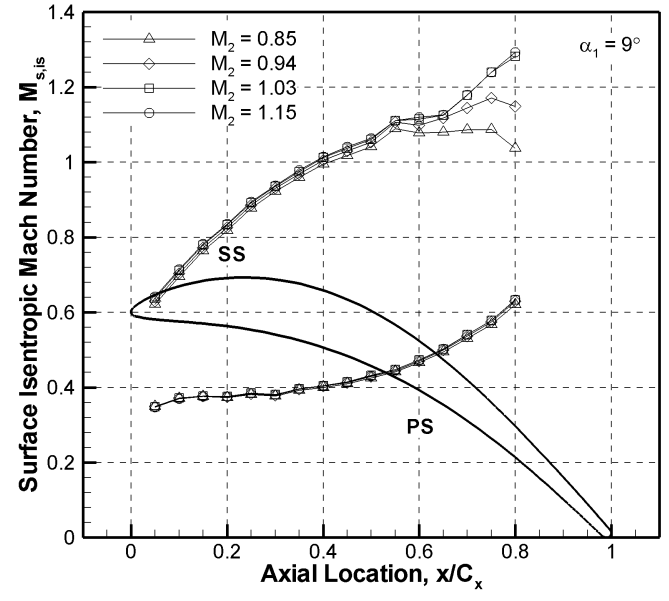

(a) $\alpha_{1}=9^{\circ}$

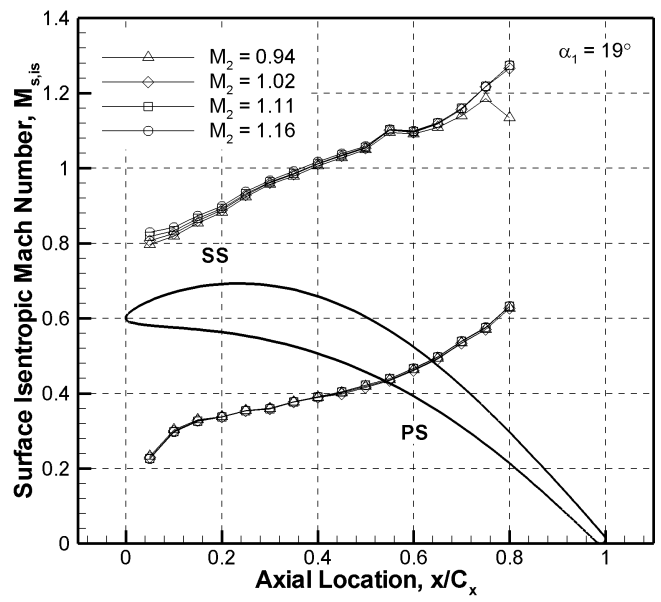

(c) $\alpha_{1}=19^{\circ}$

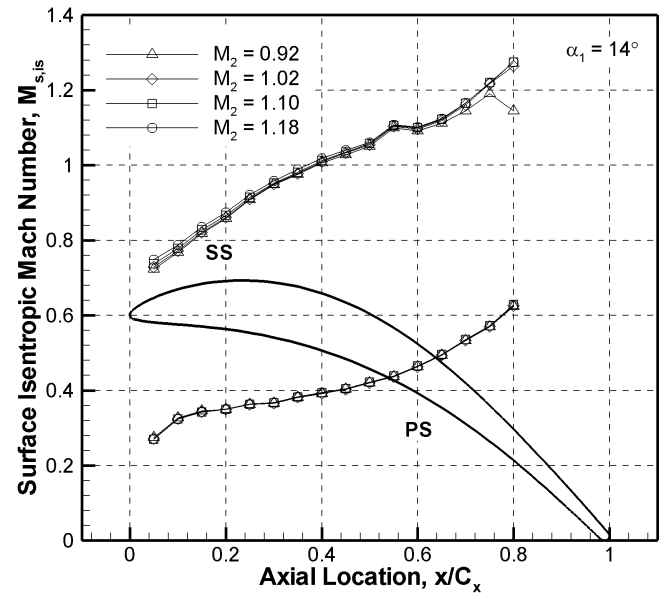

(b) $\alpha_{1}=14^{\circ}$

Figure 5.2: Surface Isentropic Mach Number $M_{s, i s}$ Distribution for E2S50 


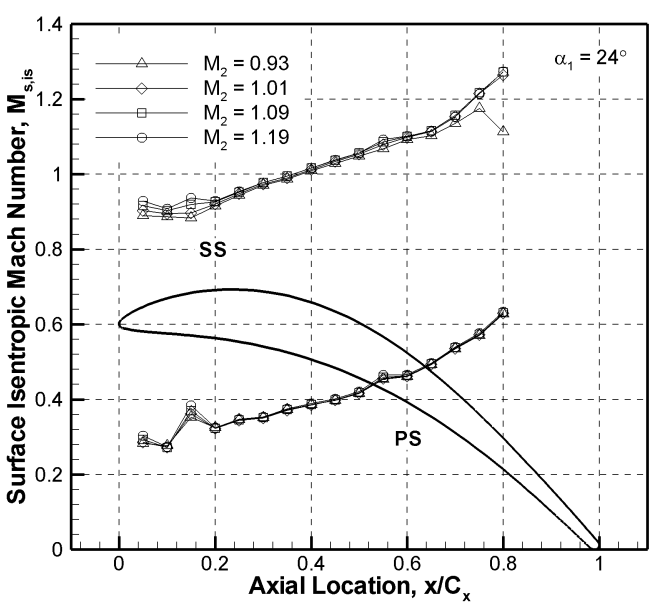

(d) $\alpha_{1}=24^{\circ}$

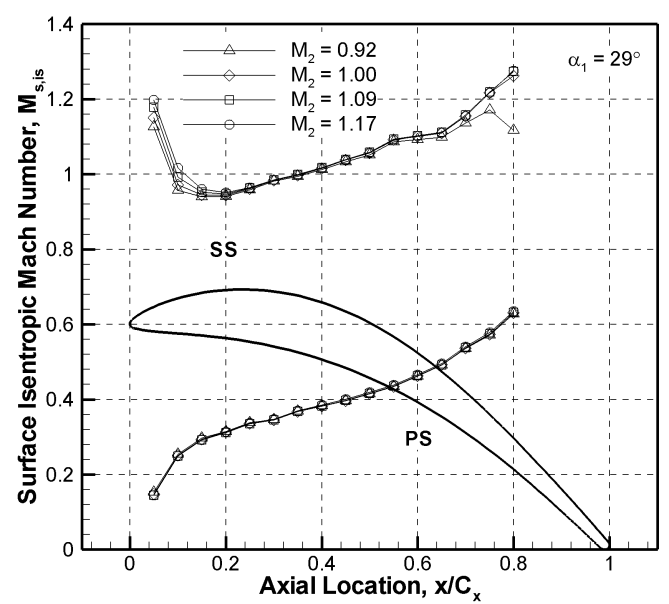

(e) $\alpha_{1}=29^{\circ}$

Figure 5.2: Surface Isentropic Mach Number $M_{s, i s}$ Distribution for E2S50 (cont.)

early onset of transition, which will increase the loss generated in the boundary layer. More discussion on the effects of surface roughness will be presented in Section 5.7,

It is only aft of about $x / c_{x}=0.55$ that the blade loadings show a dependency on outlet Mach number. At the lowest measured exit Mach number $\left(M_{2}=0.85\right)$, the suction peak is reached by $x / c_{x}=0.50$. The surface flow then remains at a constant supersonic Mach number until encountering an adverse pressure gradient aft of $x / c_{x}=0.75$. The deceleration is probably caused by an impinging shock from the adjacent blade's trailing edge. The shock wave will likely cause an increase in losses. It is possible for the boundary layer to separate as a result of the strong adverse pressure gradient caused by the shock wave-boundary layer interactions, leading to an even further increase in losses. In the unlikely event that the boundary layer has remained laminar to this point, it is likely that the adverse pressure gradient would also cause transition to occur. The trailing edge shock wave will reflect from the suction surface and propagate downstream.

As the outlet Mach number increases, the suction peak moves aft towards the trailing edge and the height of the peak increases. The second region of acceleration present aft of about $x / c_{x}=0.65$ at higher outlet Mach numbers $\left(M_{2}=0.94\right.$ and above $)$ is likely caused 


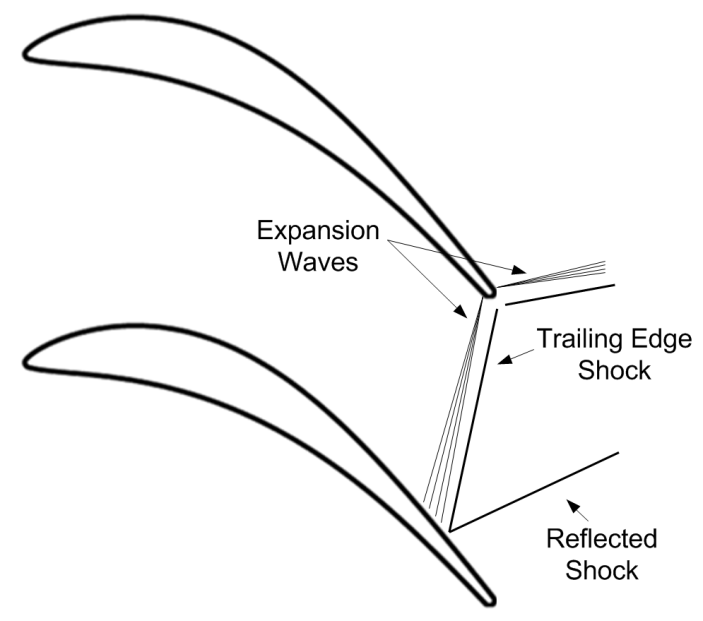

Figure 5.3: Trailing Edge Shock Structure

by expansion waves that accompany the impinging shock. The expansion waves and shock pattern are shown schematically in Figure 5.3 this is illustrative only, since the locations of the shocks and expansion waves are not known directly from the experiment. Detailed study of the trailing edge flow (Denton \& Xu, 1990 and Sieverding et al., 1979) has shown that expansion waves are formed by the change in flow direction where the separated shear layers detach and the trailing edge curvature begins. The expansion waves can propagate across the flow passage and influence the pressure distribution on the suction surface of the adjacent blade, as seen here in Figure 5.2 .

Above an outlet Mach number of about 1.0 the suction peak has moved aft of $x / c_{x}=$ 0.80. Consequently, the location of the suction peak and the shock induced deceleration are unknown. However, the suction peak and impinging shock should both move aft as the outlet Mach number increases. This agrees with the findings of Denton \& Xu (1990). As the Mach number increases, the accelerated region due to the expansion fans becomes larger and the amount of acceleration increases. Consequently, the Mach number just upstream of the impinging shock increases. However, the pressure drop across the shock does not necessarily increase because the shock becomes increasingly oblique as the outlet Mach number increases.

The flow on the pressure side accelerates over the entire measured region of the blade 
and the surface Mach number remains subsonic. Entropy generation in attached boundary layers varies as the third power of boundary layer edge velocity (i.e.: $\left.V_{\delta}^{3}\right)($ Denton, 1993$)$. Therefore, the contribution from the pressure side boundary layer to profile loss will be small in comparison to that from the suction side for this incidence.

Figures $5.2(\mathrm{~b})$ through to $5.2(\mathrm{e})$ show the surface Mach number distribution for E2S50 at different inlet angles ranging from $\alpha_{1}=14^{\circ}$ to $29^{\circ}$ in $5^{\circ}$ steps. As mentioned in Section 5.2 , blade loadings and subsequent measurements were obtained at higher values of outlet Mach number for these inlet angles compared to $\alpha_{1}=9^{\circ}$. Since results are available only up to $x / c_{x}=0.80$, at higher exit Mach numbers only one static tap for each inlet angle shows any deceleration near the aft of the blade. Hence, at the higher inlet flow angles the impinging shock has moved aft of $x / c_{x}=0.80$ at the three higher exit Mach numbers.

The pressure side results at $\alpha_{1}=24^{\circ}$ (Figure 5.2(d) show a discontinuity in the surface Mach number at about $x / c_{x}=0.05$. This is believed to be due to an error in measurement rather than a physical flow feature. It could be due to a partially blocked static tap, or more likely a leak in the measurement system. Because the entire test section is disassembled and the cascade is removed from the wind tunnel between measurements at each incidence, the blockage could have been dislodged, or the leak eliminated when the tubing was reconnected.

Near the leading edge, the loading is increased with Mach number. This effect is subtle at $\alpha_{1}=9^{\circ}$ but becomes more apparent at larger inlet angles. This is probably due to the velocity overshoot that occurs on the suction side due to the high streamline curvature as the flow rounds the leading edge.

The blade loadings for E2S50 are re-plotted in Figures 5.4(a) to 5.4(d) to aid in the discussion of the effect of incidence. Each sub-figure shows the blade loadings at all values of inlet angle at roughly the same exit Mach numbers. There are no measurements obtained near $M_{2}=1.10$ for $\alpha_{1}=9.0^{\circ}$, and therefore Figure 5.4(c) shows data for only four inlet angles. In general, the trend for loading on the forward part of the blade is similar at all exit Mach numbers.

The increased loading is a result of two phenomena. First, the flow accelerates as it turns around the leading edge; the amount of flow turning around the leading edge, and hence the 


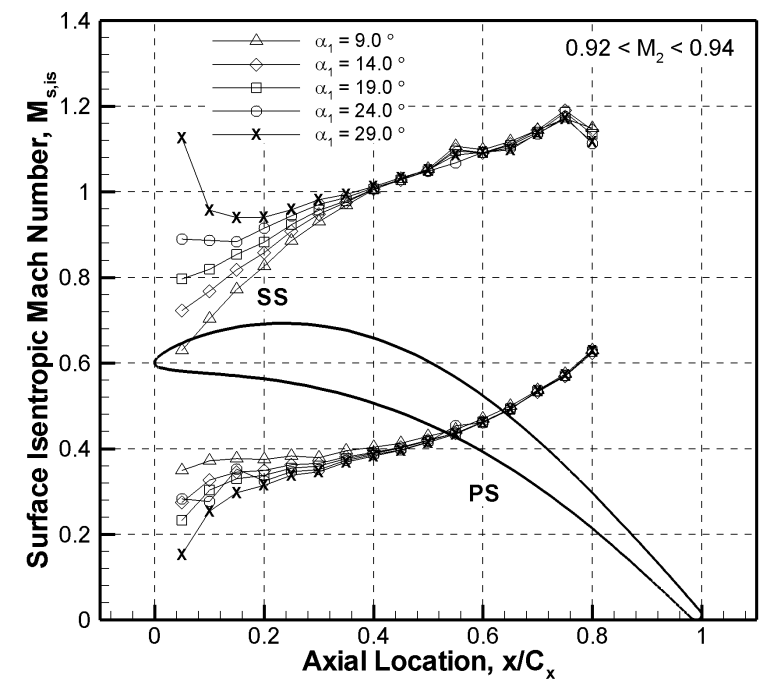

(a) $0.92<M_{2}<0.94$

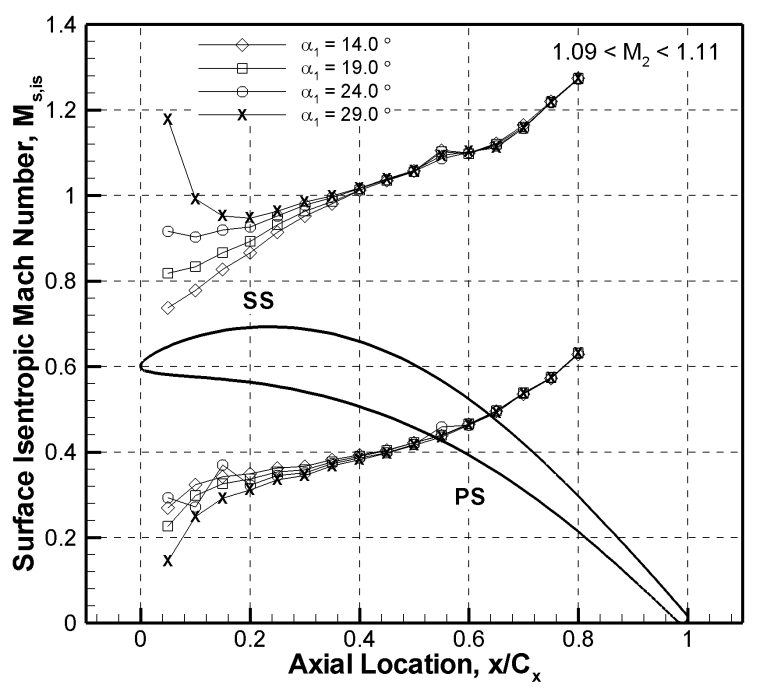

(c) $1.09<M_{2}<1.11$

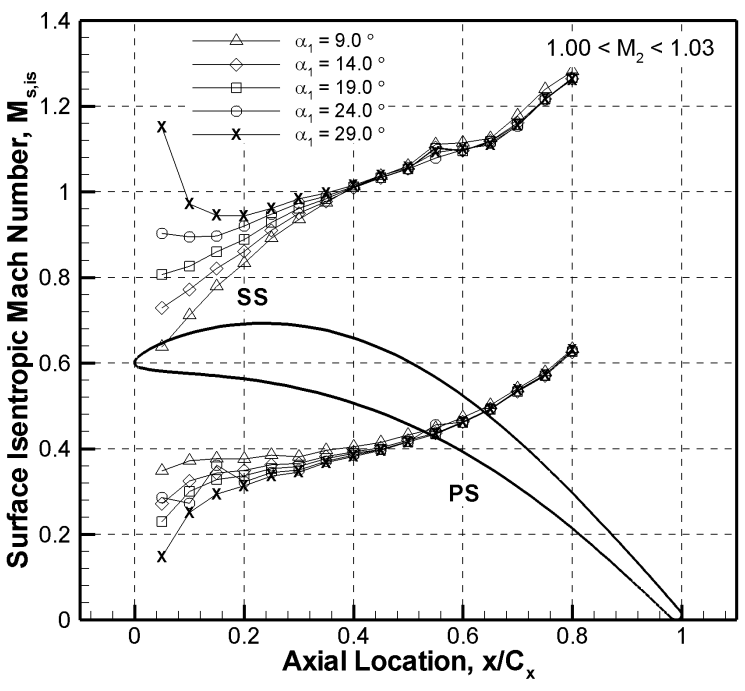

(b) $1.00<M_{2}<1.03$

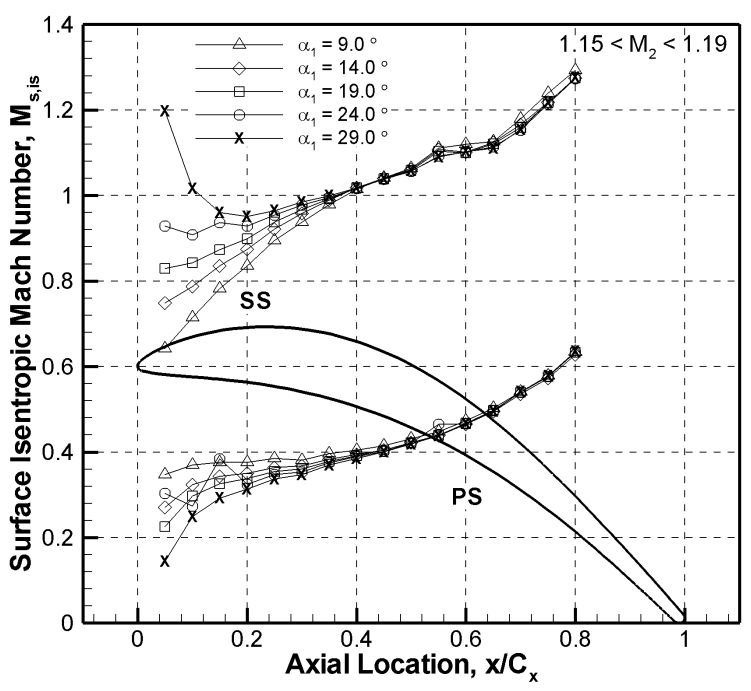

(d) $1.15<M_{2}<1.19$

Figure 5.4: Blade Loadings for E2S50 
acceleration on the suction side increases with incidence. Second, the stagnation point moves downstream on the pressure surface with increased incidence, thus increasing the local static pressure near the leading edge on the pressure side. At an inlet angle of $\alpha_{1}=29^{\circ}$, there is sufficient flow turning at the leading edge that the flow has accelerated to a supersonic velocity by $0.05 x / c_{x}$ on the suction surface. This initial acceleration is followed by a strong deceleration. Corriveau (2005) observed the same effect at positive incidence and attributed it to shocks that form on the suction surface near the leading edge. In the case of E2S50 at $\alpha_{1}=29^{\circ}$, the amount of deceleration should cause significant thickening of the boundary layer and perhaps even boundary layer separation. However, the flow then begins to accelerate at about $x / c_{x}=0.20$; the boundary layer would be expected to reattach in this favourable pressure gradient. The thicker boundary layer and separation bubble lead to increased losses which will be included in the losses measured in the downstream wake. The loadings at $\alpha_{1}=24^{\circ}$ also show deceleration on the forward part of the suction side, however, to a much lesser extent. Evidence of a thickened boundary layer can be observed in the downstream pitchwise measurements at this incidence as well, as will be discussed later.

\subsection{Experimentally Determining Design Incidence}

Later in this chapter the measured losses for the Siemens cascades will be compared to the Kacker \& Okapuu (1982) (KO) predicted losses. The KO loss system is a design incidence loss correlation, and as such, should only be compared with experimental losses obtained at design incidence. However, the design inlet flow angles for the Siemens cascades are not precisely known. Thus, measurements were not necessarily obtained at the design incidence. However, measurements were obtained at several inlet angles near the inlet metal angle $\beta_{1}$ for each cascade. The results of these off-design measurements, shown in Figure 5.5, are used to estimate the intended design incidence. The accepted sign convention used for the metal and flow angles is shown in Figure 5.5(d) using the midspan cascade E2S50 as an example.

Turbine blades are generally designed to produce the least losses when the engine is operating at the design point. This would typically occur when the gas flow angle meets the 


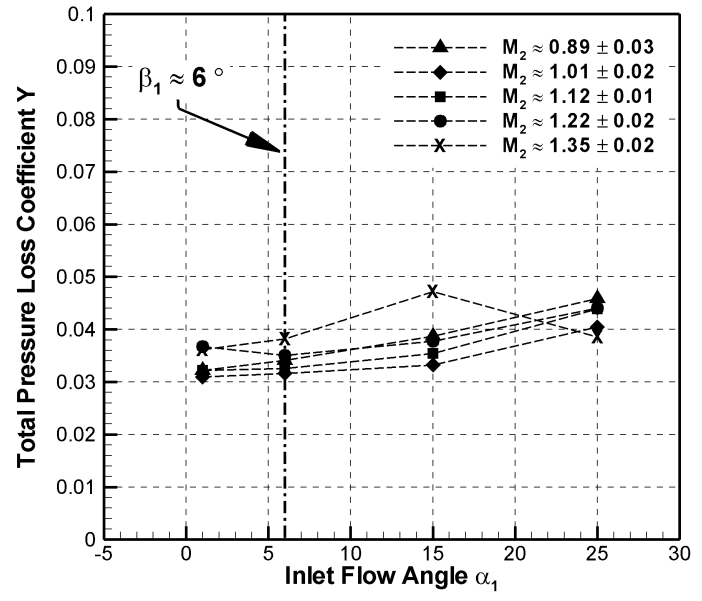

(a) Midspan Cascade E1S50

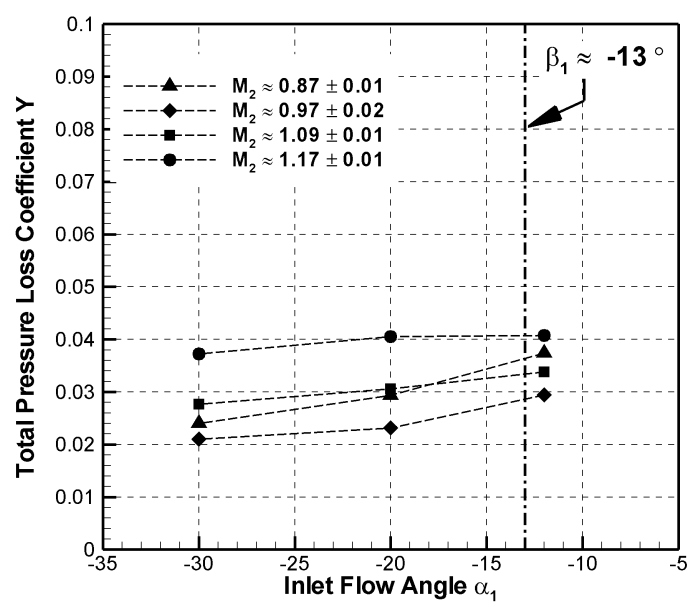

(c) Tip Section E2S90

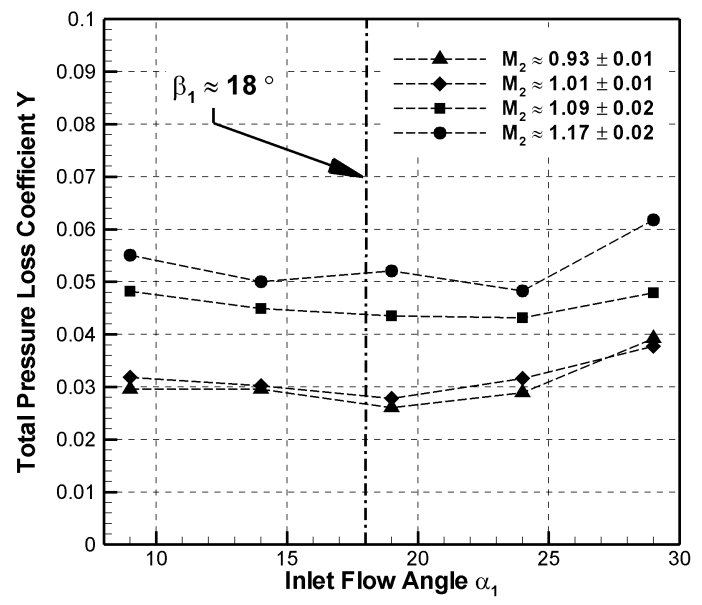

(b) Midspan Cascade E2S50

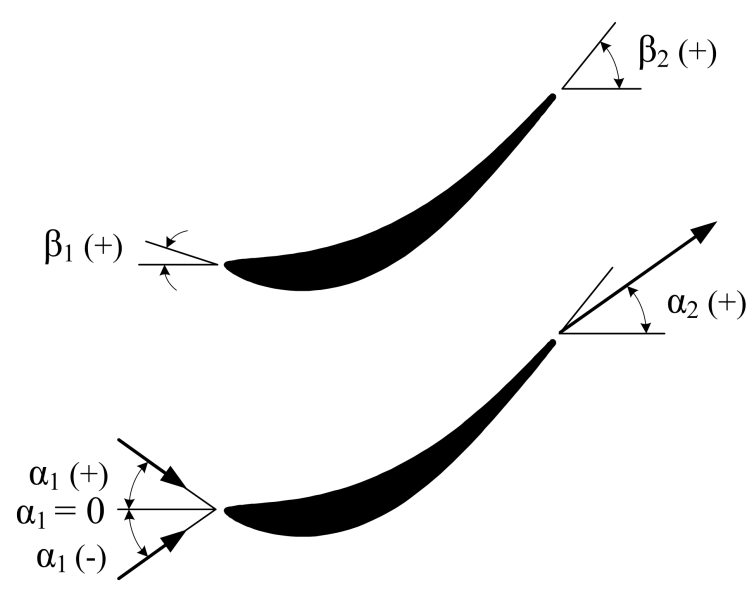

(d) Sign Convention for Metal and Flow Angles

Figure 5.5: Effect of Incidence on Losses for the Siemens Cascades 
leading edge at close to the metal angle. However, the induced velocity field upstream of the leading edge can act to increase the apparent inlet flow angle experienced by the blade. Therefore, when designing the airfoil geometry, after the flow path is designed, it is common practice to design the leading edge to have an inlet metal angle several degrees larger than that of the inlet flow angle (i.e. $\left.\alpha_{1}<\beta_{1}\right)$.

Additionally, it is sometimes favourable to use as a design inlet flow angle one that is less than the minimum loss incidence because of the asymmetrical nature of the loss bucket. As shown in Chapter 2 , losses typically increase more rapidly at positive incidences due to the potential for suction surface separation and blade stall. Thus, a slight negative bias in design incidence allows good performance over a wider range off-design inlet flow angles about the design value.

Figure 5.5 shows the variation of losses with inlet flow angle for the three Siemens cascades for which experimental measurements were obtained: the two midspan sections, E1S50 and E2S50, and the tip section E1S90. The estimated inlet metal angles $\beta_{1}$ are shown for reference. The losses are plotted against the inlet flow angle $\alpha_{1}$ at several values of outlet Mach number $M_{2}$. As described in Chapter 4, the true outlet Mach numbers are not known at the time of measurement since total pressure losses are not known a priori. The wind tunnel is controlled instead based on the isentropic Mach number obtained from the upstream total pressure and the downstream wall static pressure. Therefore, it is difficult to obtain measurements at specific intended outlet Mach numbers at different values of inlet flow angle due to the change in losses. Where data were not obtained at all inlet angles for a specific Mach number, the data were omitted from these plots. For example, losses were measured for E1S50 at an outlet Mach number of 1.41 at inlet angles of $1.0^{\circ}$ and $6.0^{\circ}$. However, at all other inlet angles for which losses were measured, the highest Mach number was no greater than 1.35. Therefore, the loss data at $M_{2}=1.41$ are not used to examine the effects incidence.

Below about $M_{2}=1.15$, the losses for E1S50 (Figure 5.5(a) increase with inlet angle over the full range measured. At these Mach numbers, it is not clear whether the losses would continue to decrease at lower values of inlet angle. However, of the inlet angles measured, the lowest losses are obtained at $\alpha_{1}=1.0^{\circ}$. As the Mach number increases the loss bucket 
changes; at about $M_{2}=1.2$, the minimum loss incidence moves to $\alpha_{1}=6.0^{\circ}$ but returns to $1.0^{\circ}$ by $M_{2} \approx 1.35$. The reason for the drop in losses at $\alpha_{1}=25^{\circ}$ and $M_{2} \approx 1.35$ is unclear, but stable operating points are more difficult to obtain as the Mach number increases.

From the loss trends shown in Figure 5.5(a), it is reasonable to take the design incidence for this cascade as close to $\alpha_{1}=0^{\circ}$ (axial flow) or possibly lower. Unfortunately, no data exists below $\alpha_{1}=1.0^{\circ}$. Therefore, data obtained at $\alpha_{1}=1.0^{\circ}$ and $6.0^{\circ}$ will both be considered to represent an approximation of the design incidence losses.

Loss measurements for E2S50 (Figure $5.5(\mathrm{~b})$ ) suggest that a the minimum loss inlet angle is probably between $\alpha_{1}=15^{\circ}$ and $25^{\circ}$. The losses are largely constant in this range, with small variations observed at $19^{\circ}$. This variation is within expected scatter. Above $25^{\circ}$, there is a sharp increase in losses, confirming the speculation of boundary layer thickening and possible separation on the suction surface that was discussed in Section 5.3. The losses also seem to rise below about $\alpha_{1}=10^{\circ}$, although the rise in losses at low inlet angles seems to be more gradual.

Figure 5.5(b) shows that the losses E2S50 are not strongly influenced by the inlet angle over a large range. This could be a related to the surface roughness present for this geometry, but it is not clear that this is the case. Based on the steep rise in losses at large inlet flow angles, and an inlet metal angle of roughly $18^{\circ}$, the design incidence of this cascade likely falls between $10^{\circ}$ and $15^{\circ}$. The closest inlet angles, at which data were collected, are $9^{\circ}$ and $14^{\circ}$. Data from these two inlet angles will therefore be compared with the design incidence predictions from Kacker \& Okapuu.

The losses for E2S90 (Figure 5.5(c) increase with flow angle, showing the lowest losses at $-30^{\circ}$. From the data, it is not clear the minimum in losses was reached. Ideally, losses would be measured at larger negative inlet angles until a rise in losses was observed. Unfortunately, the wind tunnel could not accommodate negative inlet angles below $-30^{\circ}$. Despite the higher losses at higher inlet angles, it is still likely that the intended design incidence is close to $-20^{\circ}$. This estimate is based on the fact that E2S50 and E2S90 are two sections of the same airfoil. These two sections have metal angles that differ by about $30^{\circ}$ (as a consequence of the twist of the actual blade); it follows then that the design incidences should differ by a similar 
Table 5.2: Estimated Design Incidence

\begin{tabular}{lcc}
\hline Cascade & Estimated Design Incidence & Representative Measurements \\
\hline \hline E1S50 & $-5^{\circ}<i_{d e s}<5^{\circ}$ & $\alpha_{1}=1.0^{\circ} \& 6.0^{\circ}$ \\
E2S50 & $5^{\circ}<i_{d e s}<15^{\circ}$ & $\alpha_{1}=9.0^{\circ} \& 14^{\circ}$ \\
E2S90 & $-25^{\circ}<i_{d e s}<-15^{\circ}$ & $\alpha_{1}=-20^{\circ} \&-12^{\circ}$ \\
\hline
\end{tabular}

amount. Since E2S50 likely has a design incidence no lower than $+10^{\circ}$, the design incidence for E2S90 is not likely to be less than $-20^{\circ}$. Therefore, the data collected at $-12^{\circ}$ and $-20^{\circ}$ are taken as representative of design incidence losses.

In summary, losses were not measured at the intended design incidences for the three Siemens cascades. Using the loss data measured at several inlet angles, estimates for the design incidence were made; these estimates are shown in Table 5.2. Fortunately, all three cascades do not show a strong dependence on inlet angle near the design incidence. Therefore, losses measured at the two inlet angles nearest the estimated design incidence are considered to be representative of the losses at the design incidence. The influence of Mach number on losses and outlet flow angle for these incidences will be discussed further in the following sections. Comparisons will be made with the Kacker \& Okapuu loss predictions.

\subsection{Results for the Siemens Cascades Near Design Incidence}

\subsubsection{Profile Losses}

The influence of Mach number on the total pressure loss coefficient $Y$ for the Siemens cascades is shown in Figure 5.6. For each cascade, results at two inlet angles near the estimated design incidence are shown for the reasons discussed in the last section. However, results obtained at all values of inlet angle are tabulated in Appendix C. The mixed out properties shown (e.g.: $M_{2}, Y$, etc.) have been calculated by the method of Amecke \& Šafařík (1995), as described in Section 4.4.4,

The losses of the midspan geometry E1S50 are similar for both inlet angles. This suggests 


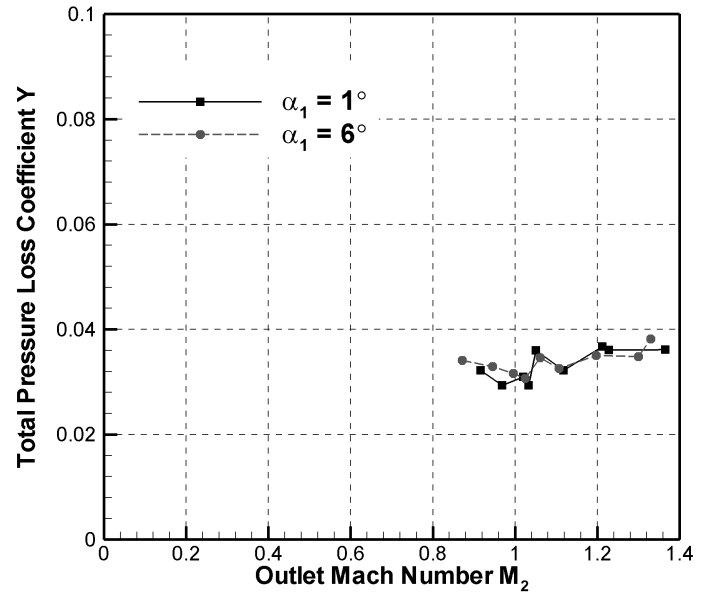

(a) Midspan Cascade E1S50

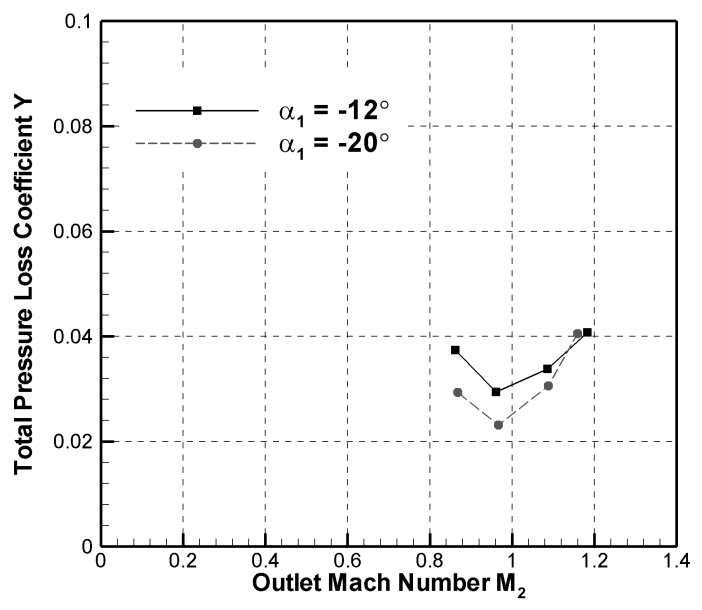

(c) Tip Section Cascade E2S90

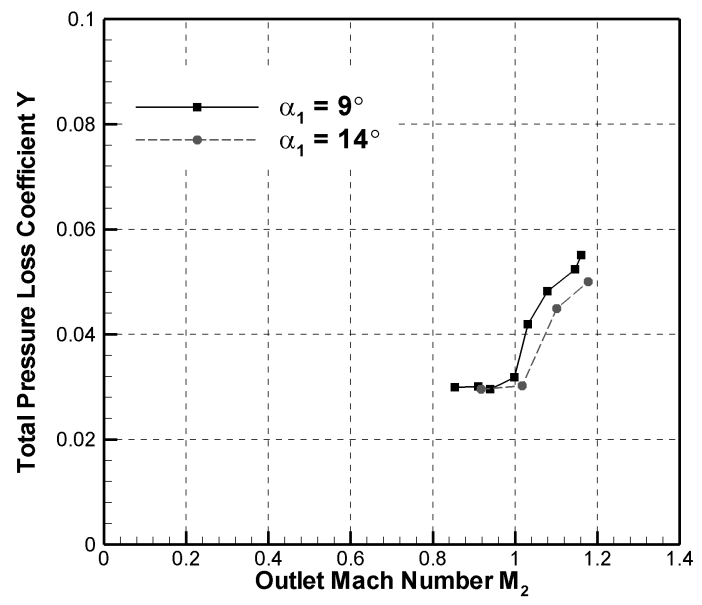

(b) Midspan Cascade E2S50

Figure 5.6: Variation of Midspan Losses with Outlet Mach Number Near Design Incidence 
that these angles are indeed close to the design incidence. It also indicates that this cascade is insensitive to small changes in inlet angle near the design incidence. Furthermore, there does not appear to be any strong dependence on Mach number. Losses decrease slightly as the outlet Mach number approaches 1.0 and there is a slight increase in losses observable within the scatter at supersonic outlet Mach numbers. The trailing edge of E1S50 is very thin; trailing edge blockage $t / o$ for this cascade is only about 0.02 . Thus, the major contribution to the overall losses is from the blade surface boundary layers and the trailing edge shock waves, and not the base pressure.

It was shown in Section 4.6.3 that cascade E1S50 is choked at outlet Mach numbers above about $M_{2}=0.90$. Once the cascade is choked, increasing the outlet Mach number does not change the surface boundary layer development upstream of the throat, which accounts for a large fraction of the blade surface. However, changes in the boundary layer downstream of the throat, where increases in the outlet Mach number cause the boundary layer on this portion of the blade to thin, will influence the profile losses. Above about $M_{2}=1.0$, the impinging trailing edge shock will interact with the surface boundary layer, further influencing the losses. The increase in Reynolds number associated with the increase in Mach number will have a small effect on the boundary layer development over the entire blade surface.

The lack of a significant increase in losses above Mach 1.0 would suggest that the shock losses do not contribute greatly to the overall losses. The trailing edge shock system is formed due to the sudden expansion and resulting compression at the trailing edge. For E1S50, the expansion at the trailing edge is probably small and the resulting shocks weak. As the Mach number increases, the shocks become increasingly oblique. Total pressure loss across the shocks decreases with the increasing obliquity of the shocks.

Unfortunately, no blade loading measurements exist for E1S50. Blade loadings would have helped to determine the strength of the impinging trailing edge shock. However, the variation of the total pressure loss downstream of the cascade, shown in Figure 5.7 for E1S50, offers a means of determining the contributions from the shocks. Figure 5.7 shows the pitchwise variation of losses downstream of the cascade at $\alpha_{1}=1.0^{\circ}$ and $\alpha_{1}=6.0^{\circ}$ at several Mach numbers between 0.9 and 1.2 . 


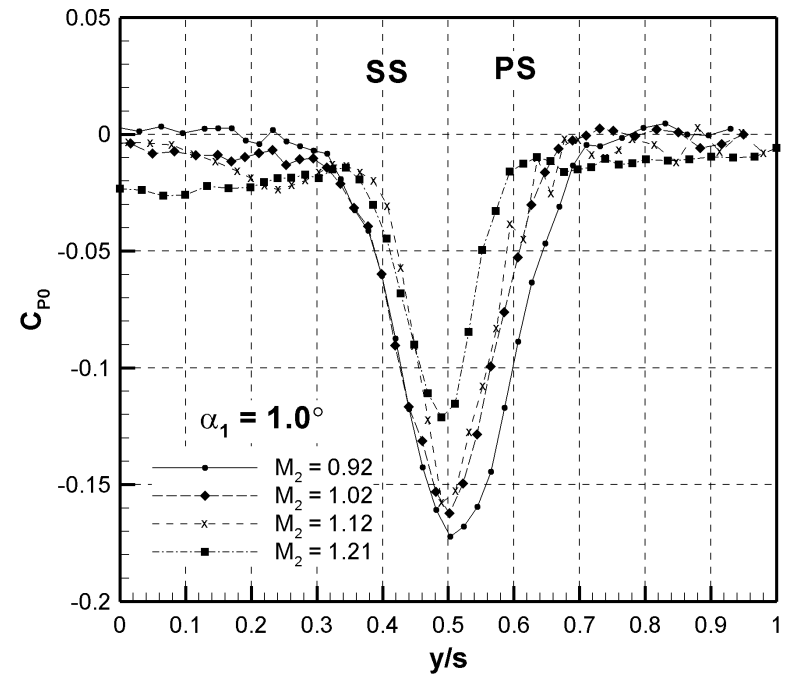

(a) $\alpha_{1}=1.0^{\circ}$

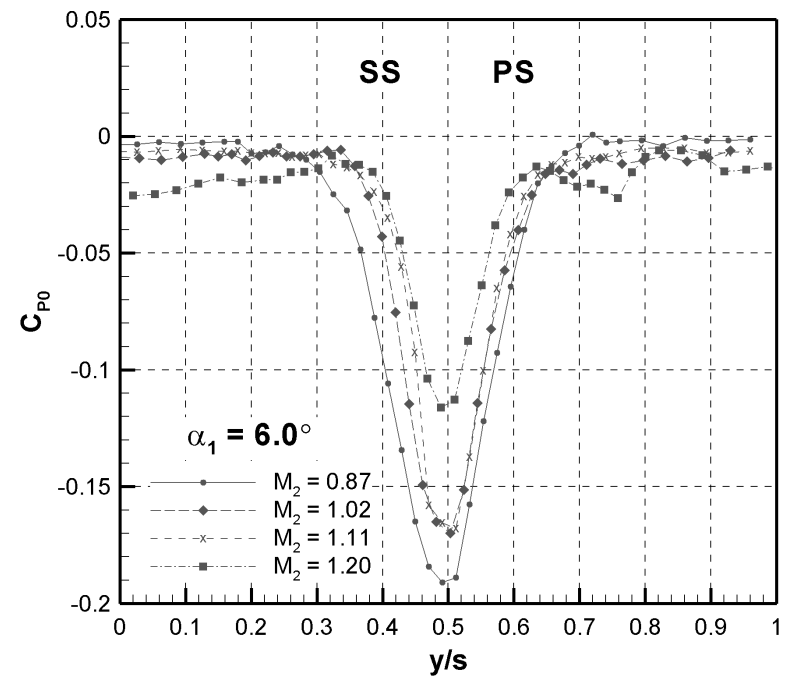

(b) $\alpha_{1}=6.0^{\circ}$

Figure 5.7: Pitchwise Variation of Total Pressure Loss $C_{p o}$ for E1S50

There are no obvious differences in the wakes between the two values of incidence. Both the depth and width of the wake of E1S50 decrease as $M_{2}$ increases. Since the trailing edge losses are expected to be small, the thinner wakes are probably a result of thinner surface boundary layers. As mentioned, the increases in both Mach and Reynolds numbers thins the boundary layers.

Shock losses can be estimated by averaging the loss coefficient in the mid-passage region outside the wakes. Since the measurements are obtained $0.5 c_{x}$ downstream of the trailing edge, losses from both of the trailing edge shocks, and the shock reflected from the suction surface will contribute to the mid-passage losses. Furthermore, as the shocks propagate downstream they will cross the measurement plane. Therefore, measurements over a certain fraction of the pitch could have been obtained downstream of a particular shock, while the remaining measurements for this pitch would have been obtained upstream of the same shock. This would manifest as a sudden rise or drop in mid-passage losses near the location at which the shock crosses the measurement plane.

The mid-passage losses are zero at about $M_{2}=0.90$ and lower. As the outlet Mach 
number increases, so too do the losses. In the case of E1S50 the decrease in losses associated with the wakes is roughly balanced by the increase in mid-passage losses. This gives rise to the seemingly constant loss behaviour indicated by Figure 5.6(a). However, this is not a universal behaviour, as evident from the other cascades. For example, the loss behaviour of the other midspan geometry, E2S50 (Figure 5.6(b) is quite different. These cascades differ in several ways. Among other things, E2S50 is not hydraulically smooth. Furthermore, the trailing edge blockage for E2S50 is more than twice that of E1S50 at $t / o=0.45$.

Between about $M_{2}=0.8$ and 1.0, the losses of E2S50 are constant. The losses rise rapidly at low supersonic Mach numbers. The losses appear to be leveling off in a plateau as $M_{2}$ approaches 1.2. Interestingly, the rise in loss at about $M_{2}=1.0$ does not appear to be the result of just increased shock losses. The downstream pitchwise variation of loss is shown in Figure 5.8. Although the mid-passage losses do increase with Mach number, this effect is small in comparison to the losses associated with the wake. The shock losses appear to be largest on the pressure side of the wake.

In addition to the shock losses, the wake of E2S50 also increases in depth with Mach number and the width of the wake increases slightly. The variation in the depth of the wake for E2S50 is small compared to the depth of the wake itself. The surface roughness of E2S50 is likely the cause of this. Aungier (2006) notes that the skin friction coefficient $c_{f}$ becomes roughly independent of Reynolds number if the roughness elements penetrate beyond the laminar sublayer of the boundary layer. Therefore, increasing the Reynolds number past the point where roughness effects begin to dominate does not significantly change the losses. Though individually each change is small, the increases in shock losses, wake depth and wake width all result in the sharp rise in losses shown in Figure 5.6(b).

Figure 5.6(c) shows the losses of the tip section cascade E2S90 as a function of Mach number. The subsonic losses of this cascade decrease as the Mach number approaches 1.0. Above $M_{2}=1.0$ the losses increase with Mach number. The pitchwise variation of $C_{p o}$ for E2S90 is shown in Figure 5.9 .

At the lowest Mach number at $\alpha_{1}=-12^{\circ}$, the wake for E2S90 is large and asymmetric. The increased losses on the suction side suggests that there may be a laminar separation 


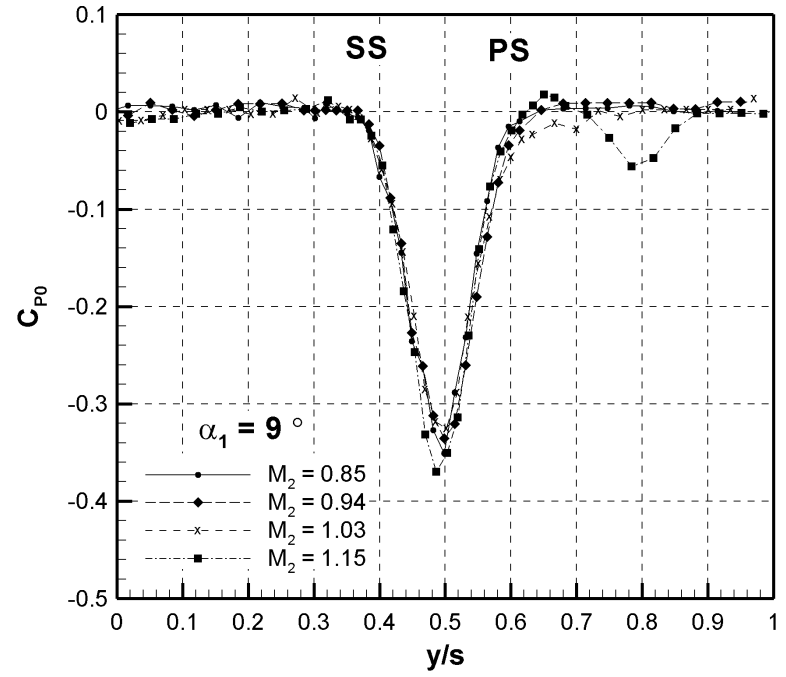

(a) $\alpha_{1}=9^{\circ}$

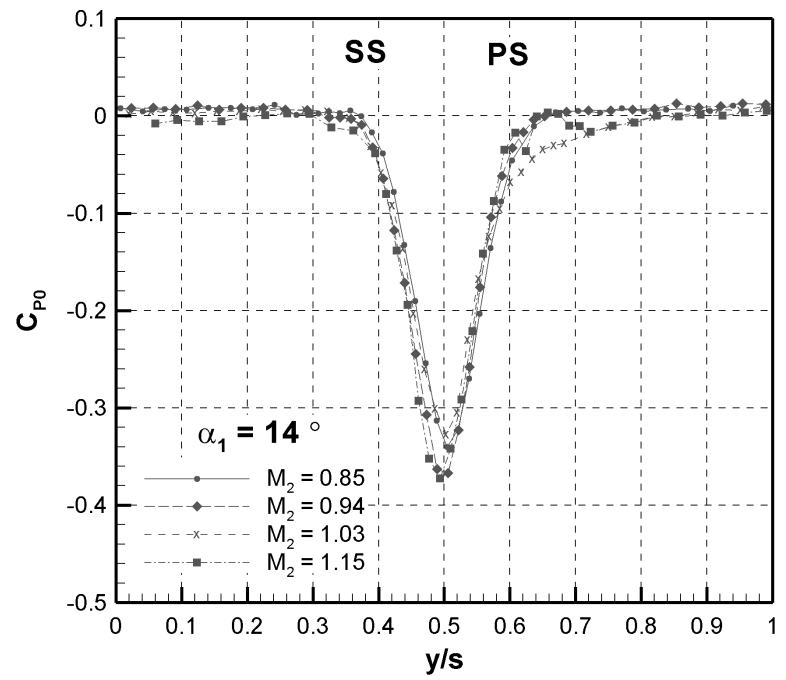

(b) $\alpha_{1}=14^{\circ}$

Figure 5.8: Pitchwise Variation of Total Pressure Loss $C_{p o}$ for E2S50

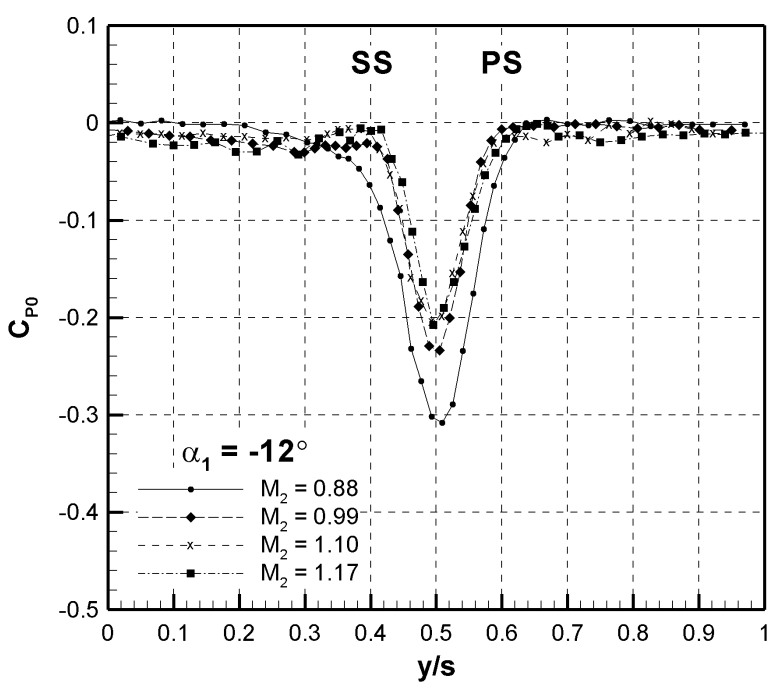

(a) $\alpha_{1}=-12^{\circ}$

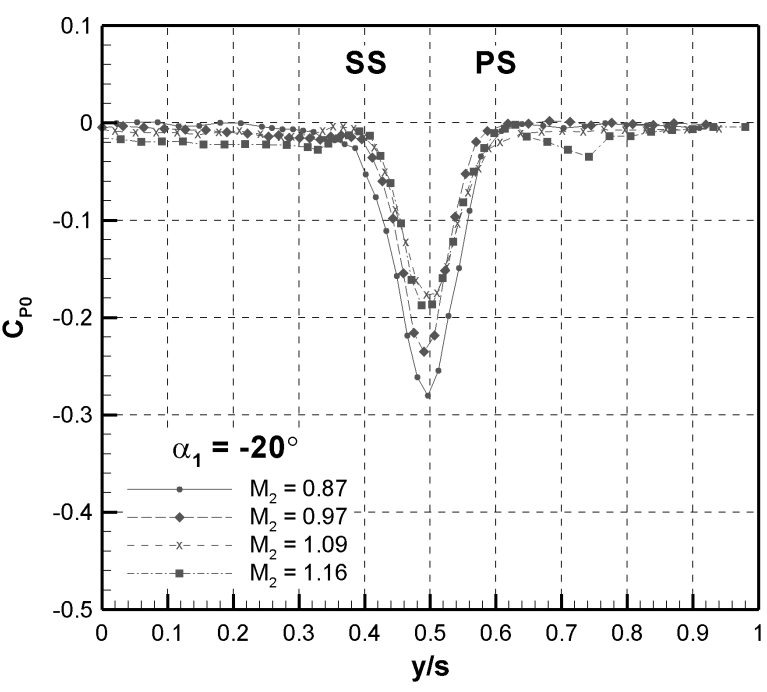

(b) $\alpha_{1}=-20^{\circ}$

Figure 5.9: Pitchwise Variation of Total Pressure Loss $C_{p o}$ for E2S90 
bubble present. This is further supported by the drop in losses at higher Mach numbers, hence higher Reynolds numbers. If the increase in Reynolds number is sufficient, transition to turbulence will occur before the laminar boundary layer separates, reducing losses.

The asymmetry in the wake is less apparent at $\alpha_{1}=-20^{\circ}$. Unfortunately, no blade loading measurements are available for this cascade. However, from the blade loadings of E2S50, it was shown that increasing the inlet angle increases the loading at the leading edge. If the inlet angle is increased sufficiently, regions of strong deceleration near the leading edge can form. If E2S90 behaves in such a way at $\alpha_{1}=-12^{\circ}$, it is possible that the deceleration at the leading edge causes a short separation bubble to form. Decreasing the inlet angle to $-20^{\circ}$ may be sufficient to suppress such a separation.

Similar to the midspan cascades, E2S90 begins to show signs of shock losses above about $M_{2}=1.0$. The wake continues to decrease in size at supersonic outlet Mach number as well. However, the increase in shock losses is greater. The overall result is an increase in losses with Mach number at supersonic conditions.

\subsubsection{Outlet Flow Angle}

Flow deviation at the trailing edge of the cascade is important to performance. The amount of work done by a turbine stage is proportional to the amount of flow turning. It is therefore important to understand the variation of the the outlet flow angle at off-design conditions. Flow angles were measured using the three-hole probe (THP) described in Section 3.4.1.

The mixed-out values of the outlet flow angles for the Siemens cascades are shown in Figure 5.10 as a function of Mach number. Results at two inlet angles near the estimated design incidence are shown. The estimated outlet metal angles for the cascades are indicated on the figures.

The trend of $\alpha_{2}$ is the similar for all three cascades: $\alpha_{2}$ increases to a maximum near $M_{2}=1.0$, above which the flow angle decreases. As the outlet Mach number becomes supersonic, the flow must turn towards the axial direction (reducing the flow angle) to increase the flow area and thus create a convergent-divergent streamtube. The most obvious difference 


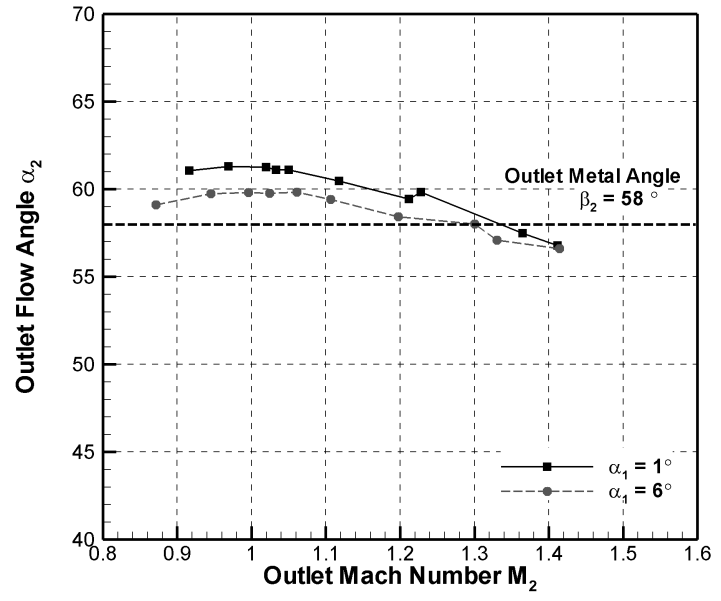

(a) Midspan Cascade E1S50

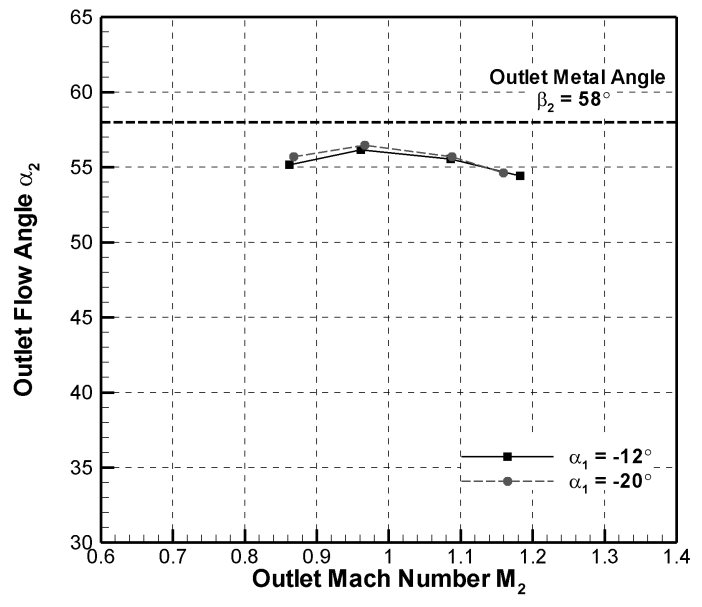

(c) Tip Section Cascade E2S90

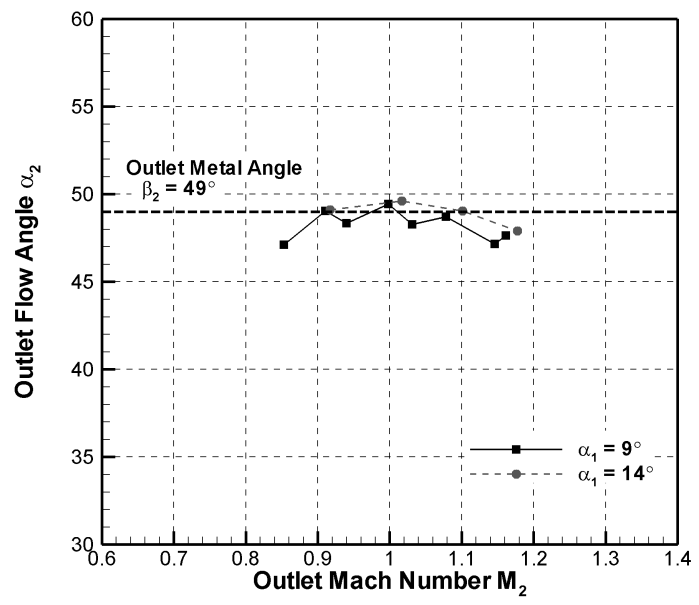

(b) Midspan Cascade E2S50

Figure 5.10: Variation of Outlet Flow Angle $\alpha_{2}$ with Mach Number Near Design Incidence 


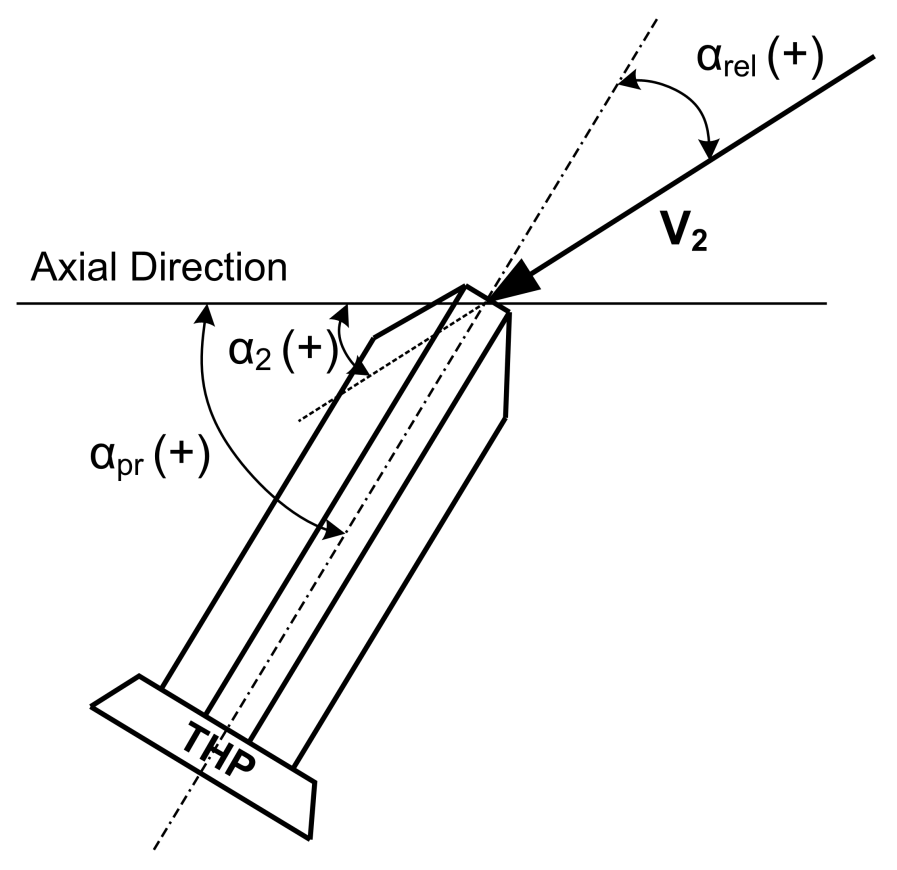

Figure 5.11: Schematic of Probe Tip and Flow Angles

between the three cascades is that the flow angle exceeds the metal angle for E1S50.

Figure 5.10(a) shows that the flow angle for E1S50 is larger than the metal angle for this cascade. This is referred to as overturning. This is not what is usually expected. Typically, the higher pressure on the pressure side drives the flow towards the low pressure on suction side, reducing the flow angle. Several factors could lead to this phenomenon of over-turning.

A potential source of error is the measurement of the angle at which the probe was positioned in the wind tunnel $\alpha_{p r}$. Using the three hole probe calibrations, a flow angle is measured relative to the probe $\left(\alpha_{r e l}\right) . \alpha_{2}$ is then calculated as follows:

$$
\alpha_{2}=\alpha_{p r}-\alpha_{r e l}
$$

Figure 5.11 is a schematic representation of the probe and the sign conventions for the various angles.

The probe angle is set using a protractor. Any error introduced in setting this angle will result in a uniform shift of the angle data at each incidence. It is believed that the uncertainty 


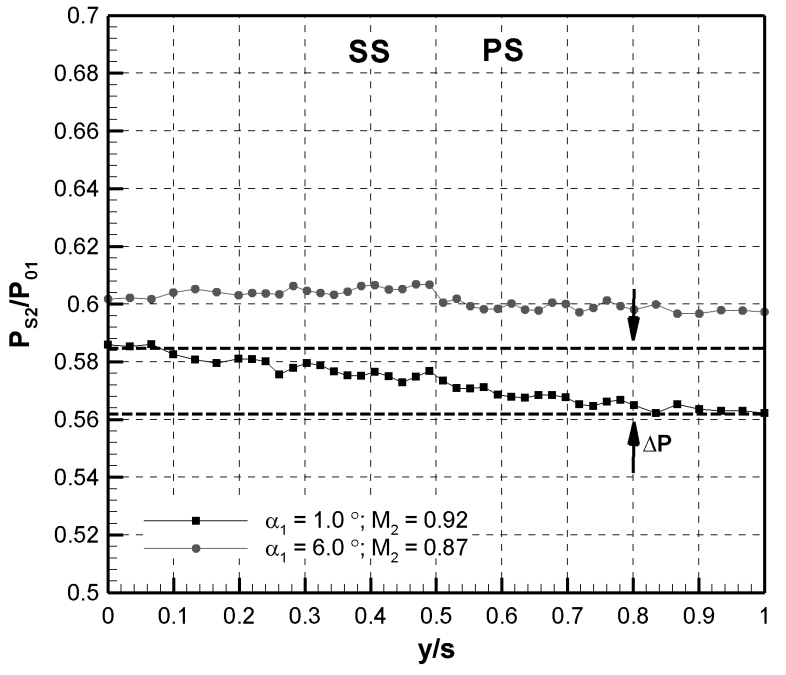

(a) $M_{2} \approx 0.9$

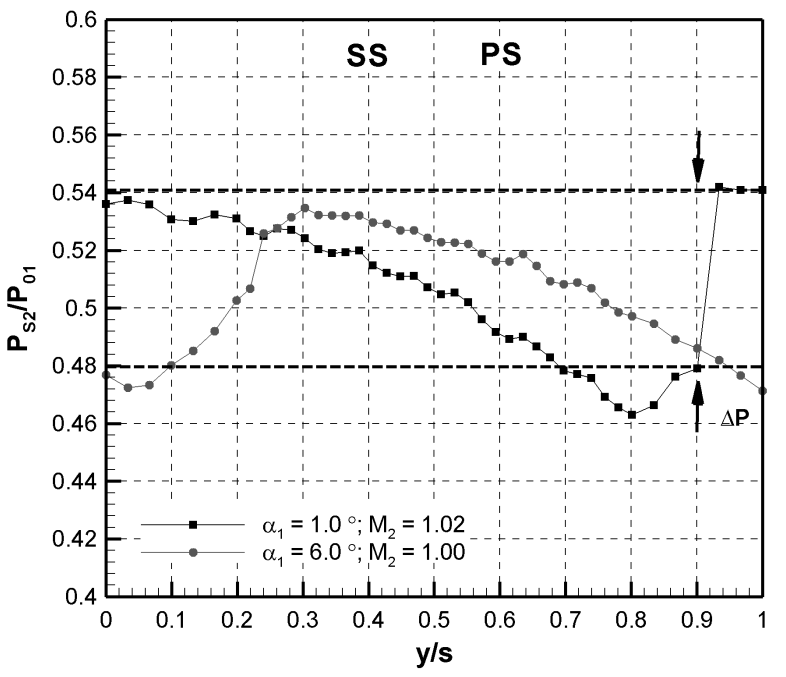

(b) $M_{2} \approx 1.0$

Figure 5.12: Pitchwise Variation of Downstream Static Pressure for E1S50 Obtained with the Static Pressure Probe

in setting $\alpha_{p r}$ by this method is about $\pm 1.0^{\circ}$. The uncertainty in the reduced angles from the three hole probe calibration $\left(\alpha_{r e l}\right)$ is $\pm 0.5^{\circ}$ for relative flow angles between $-10^{\circ}$ and $+10^{\circ}$ (see Corriveau, 2005). This amount of uncertainty does not seem to account for the over-turning shown in Figure 5.10(a).

In order for the flow to turn through a larger angle than the metal angle, there must exist a force, apart from the blade itself, turning the fluid. That is, there must be a pressure gradient driving the fluid. Therefore, the static pressures distribution is examined.

Figures $5.12(\mathrm{a})$ and $5.12(\mathrm{~b})$ show the downstream static pressure $P_{2}$ normalized by the upstream total pressure $P_{01}$ for E1S50 at $M_{2} \approx 0.9$ and $M_{2} \approx 1.0$, respectively. These two Mach numbers are shown because the results at $M_{2} \approx 0.9$ show the static pressure distribution in the absence of shocks, and the results at $M_{2} \approx 1.0$ corresponds to the case which shows the most overturning.

Figure 5.12 shows that there is indeed a pressure gradient, however only at $\alpha_{1}=1.0^{\circ}$. This inlet angle shows the most over-turning. At $M_{2} \approx 0.9$ the static pressure steadily declines across the pitch. At $M_{2} \approx 1.0$, the static pressure difference $\Delta P$ between the suction side 
and pressure side of the passage is clear; at about $y / s=0.9$, where the data was shifted, the static pressure increases suddenly. Recall that the measurements from the static pressure probe must be shifted in order to align the data between the static pressure probe and the three hole probe. $\Delta P$ is larger at the higher Mach number.

The cause of the pitchwise pressure gradient is unknown, however it may be caused by an undetected leak in the wind tunnel towards the suction side of the cascade. Where there is a leak, air from the surroundings will enter the test section at ambient pressure (higher than $P_{2}$ ). Such leaks have occurred in previous testing, but they are generally detected and the sealing of the test section improved to eliminate them. Unfortunately, the seals are broken each time the test section is opened to change the cascade or incidence.

The presence of a static pressure gradient downstream of the cascade should not greatly affect the losses. However, it will affect the total pressure measured by the three hole probe. Above about $M_{2}=1.0$, a bow shock forms ahead of the probe tip. The probe measures low total pressure as a consequence of the losses introduced by the shock. As the static pressure is decreased (i.e. the local Mach number increases), the total pressure drop across the shock increases. For this reason, the total pressure measured by the three hole probe is corrected using the Rayleigh Pitot formula. The formula relates the total pressure measured by the three hole probe $P_{o}^{\prime}$ to the static pressure measured by the static probe $P$, as a function of Mach number upstream of the shock $M_{\infty}$ as follows:

$$
\frac{P_{o}^{\prime}}{P}=\left[\frac{(\gamma+1) M_{\infty}^{2}}{2}\right]^{\frac{\gamma}{\gamma-1}}\left[\frac{\gamma+1}{2 \gamma M_{\infty}^{2}-(\gamma-1)}\right]^{\frac{1}{\gamma-1}}
$$

The Rayleigh Pitot formula is solved iteratively for $M_{\infty}$. The total pressure drop across the bow shock is then found by considering the portion of the shock just ahead of the three hole probe to be a normal shock. The Rayleigh pitot correction is discussed in further detail in Section 4.4 .2 ,

The cascades E2S50 and E2S90 do not show any over-turning. The flow angle for E2S50, however, shows an unexpected zig-zag pattern at $\alpha_{1}=9.0^{\circ}$. This can be explained by the fact that the data for this inlet angle was not all measured at the same time. As mentioned previously, the original measurements for E2S50 were not obtained at the targeted outlet Mach 
numbers. Consequently, data at this inlet angle was collected again with modified operating conditions. It is possible that the anomalous pattern in the E2S50 data at $\alpha_{1}=9.0^{\circ}$ is caused by differences in probe angle between the original measurements and the repeated measurements.

\subsubsection{Reynolds Number Variations}

As mentioned previously, in studies such as this one, it is difficult to separate Mach number

effects from Reynolds number effects since the Reynolds number also increases as the blowing pressure is raised to increase the Mach number. Reynolds number is based on the outlet flow conditions and the true chord length and is defined as

$$
R e_{c}=\frac{\rho_{2} V_{2} c}{\mu}
$$

The Reynolds number variations for the Siemens cascades are shown as functions of Mach number in Figure 5.13. At any given Mach number, the cascade E2S50 shows the highest Reynolds number. The influence of Reynolds numbers will be discussed further in Section 5.7 .3 .

\subsection{Kacker \& Okapuu Prediction for Siemens Cascades}

A full description of the Kacker \& Okapuu loss system (KO) is presented in Chapter 2 . This section compares the loss prediction from the KO system with the measured profile losses for the Siemens cascades.

$\mathrm{KO}$ is intended as a loss prediction method for design incidence, and as such should only be compared with experimental results taken at the design incidence. As mentioned in Section 5.4. the design inlet flow angles are not known precisely for these cascades. Thus, measurements were not necessarily obtained at the design incidence. Consequently, experimental results are presented, where available, at the two inlet flow angles that are believed to be closest to the design inlet flow angle.

Figure 5.14 shows the predicted profile losses as a function of Mach number for the four 


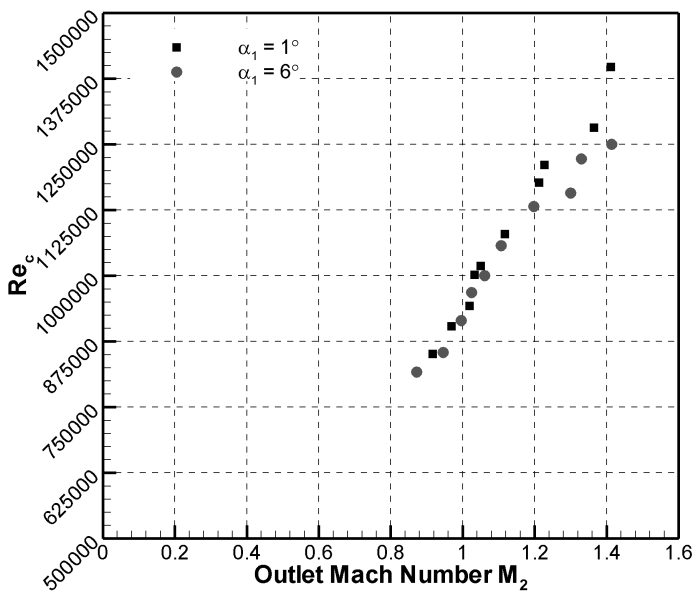

(a) Midspan Cascade E1S50

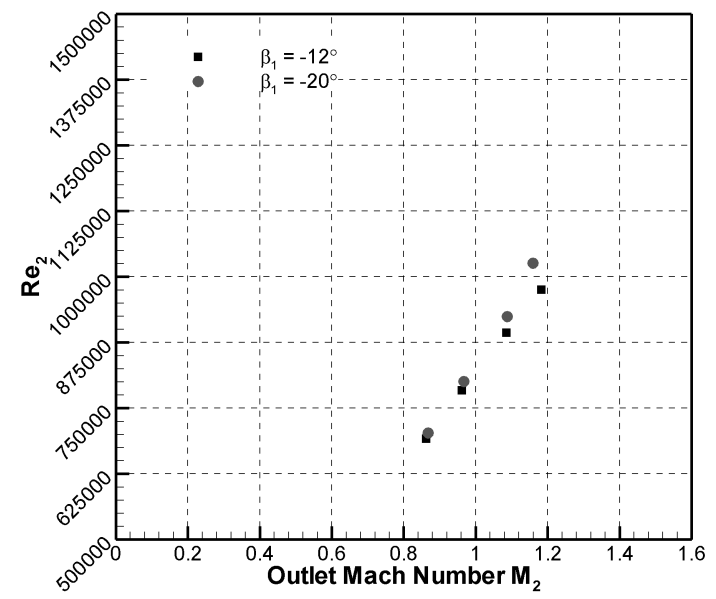

(c) Tip Section Cascade E2S90

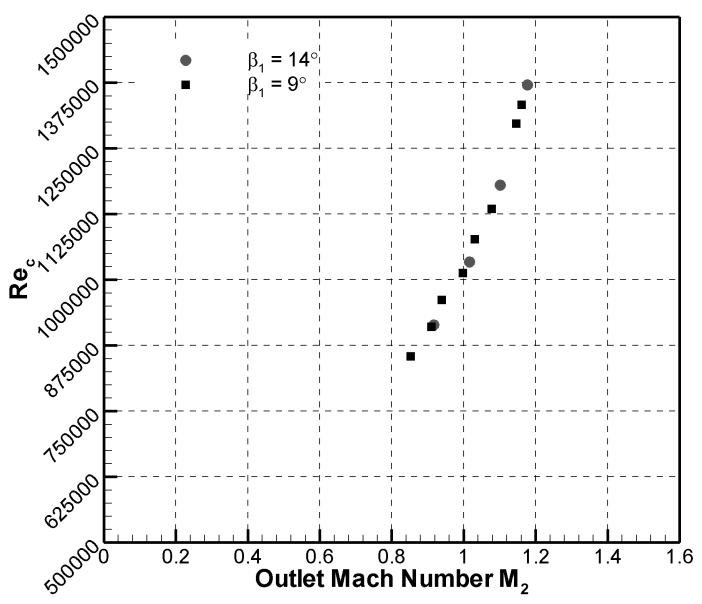

(b) Midspan Cascade E2S50

Figure 5.13: Variation of Outlet Reynolds Number $R e_{c}$ with Mach number Near Design Incidence 
Siemens cascades. The corresponding measured losses are shown with symbols. No experimental data are available for cascade E1S90, the tip section with large negative inlet design angle. Nevertheless, the KO predictions are included for this case (Figure 5.14(b) because they highlight a shortcoming of the KO loss system that will be discussed further. The predictions have not been corrected for Reynolds number since most of the measurements fall in the range where the KO Reynolds number correction (Equation 2.22) is 1.0 or close to it. It should also be noted that the E2S50 cascade was not hydraulically smooth. No roughness correction was applied to the predictions for it, but the implications of surface roughness will be discussed further in Section 5.7. Finally, two representative airfoils are shown for each cascade with the correct proportions and spacing.

In general, the KO predictions for all four cascades follow similar trends: largely constant losses for most of the subsonic Mach number range, with a small drop in losses just below Mach 1.0, and then a sharp rise in losses at supersonic Mach numbers. The experimental results, however, show different trends that vary to some degree with the cascade.

Comparing the subsonic experimental data to the predictions shows that the losses are underpredicted for all three measured cascades. For the slightly rough cascade, E2S50, this difference might have been attributed to the effects of roughness, but even the smooth cascades show apparent underprediction. Since the Reynolds numbers for all measurements are above 700,000 and in some cases cases exceed $1 \times 10^{6}$, including a Reynolds number correction would leave most predictions unchanged and, for the highest Reynolds numbers, even decrease the predictions. The predicted losses have two components: the profile loss due to the blade surface boundary layers, and the trailing edge loss due to the base pressure and the finite thickness of the trailing edge. As seen, the trailing edge losses are predicted to be very small for all of these cascades, due to their very thin trailing edges. Thus, the predicted losses are dominated by the profile contribution. Nevertheless, the overall underprediction could be due to some amount of underprediction of the trailing edge component in the KO loss system. It is not possible to determine whether this is the case from the present data.

In some respects, the most interesting subsonic predictions are for the E1S90 cascade 


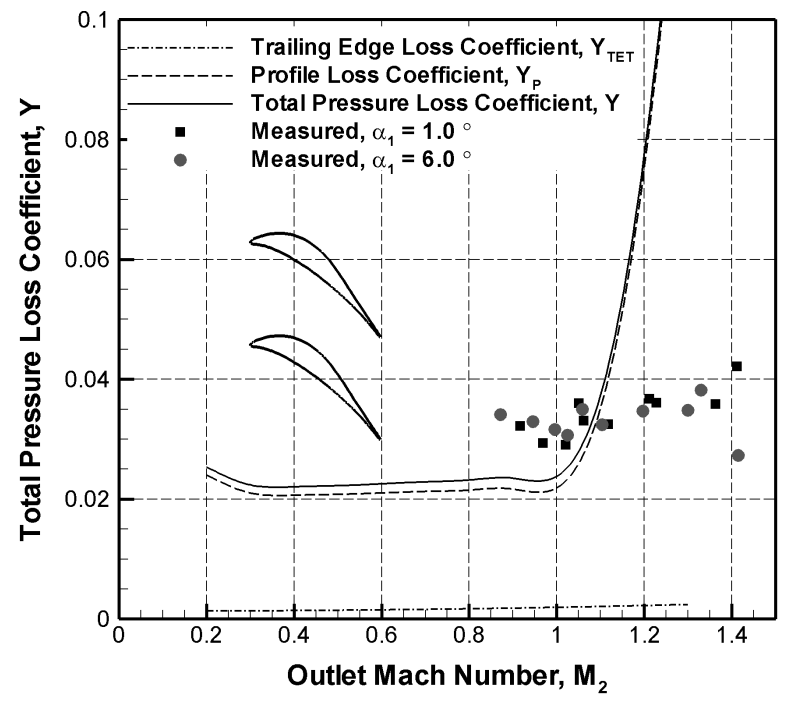

(a) E1S50

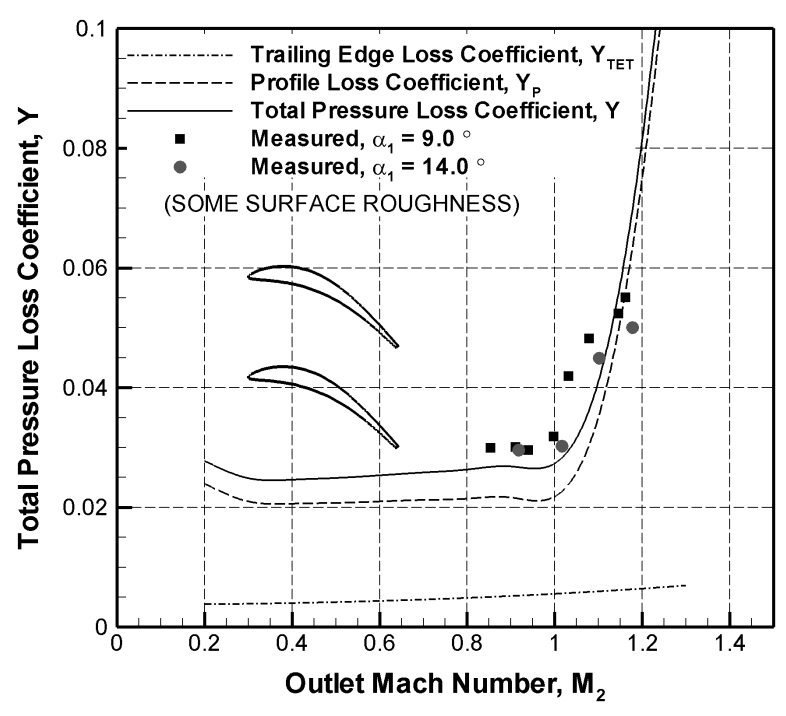

(c) E2S50

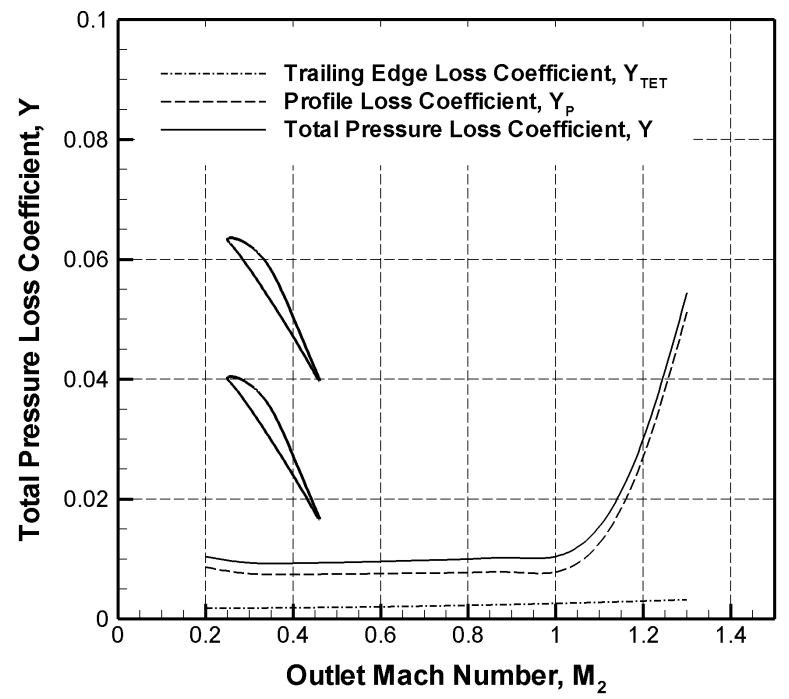

(b) E1S90

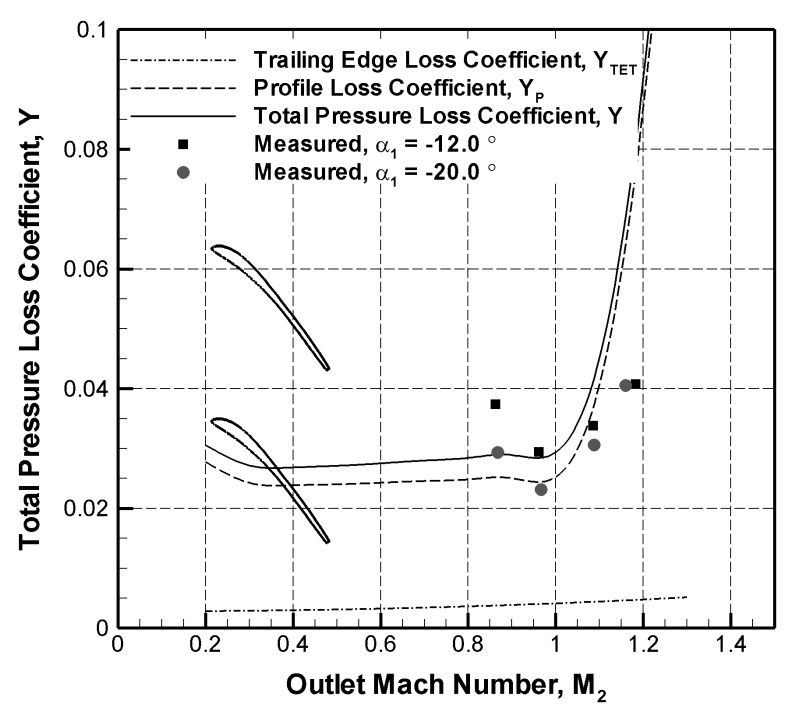

(d) E2S90

Figure 5.14: Kacker \& Okapuu Predicted Losses for Siemens Cascades 
(Figure 5.14(b)], which unfortunately was not manufactured and for which there are no experimental results. As seen, this is a tip section with a relatively low turning $\left(34^{\circ}\right)$ and a large negative inlet flow angle $\left(-32^{\circ}\right)$. The predicted losses seem implausibly low: significantly less than half the values predicted for the other three cascades. It should be noted that the KO loss system was specifically developed for meanline analysis, and thus was not intended to be applied to tip sections. At the meanline, inlet flow angles are typically positive, or at most slightly negative. While KO did allow for negative inlet flow angles, they anticipated that these would be small in magnitude. The other tip section, E2S90 (Figure 5.14(d)), also has a negative inlet angle but it is small enough in magnitude $\left(-13^{\circ}\right)$ that the predictions still look reasonable. However, the results for E1S90 suggest that there may be a fundamental flaw in the way that the KO system handles large negative inlet angles. This issue is considered to be of interest for future study, and as such will be discussed in Section 5.8 .

The Kacker \& Okapuu loss system predicts a small dip in losses as $M_{2}=1.0$ is approached. For the two midspan sections, E1S50 and E2S50, such slight dips may just be discernible within the scatter in the data. For the tip section, E2S90, there is a more obvious dip and it appears to be larger in magnitude than predicted by KO. The reason for the observed differences are not clear.

For supersonic outlet flow, KO predicts a rapid and monotonic rise in the losses. Such a rise did appear to occur for one of the midspan sections, E2S50. However, for the other midspan geometry, E1S50, the losses remained nearly constant from $M_{2}=1.0$ to about $M_{2}=1.4$. Supersonic plateaus in losses, of varying extents, have been reported in the literature and in previous studies conducted at Carleton (e.g. Corriveau, 2005). The results for the midspan section, E2S90, also hint at such a plateau. The reasons for this plateauing behaviour are not clear, although there seems to be some influence of the magnitude of surface curvature on the suction side of the airfoil downstream of the throat. Another thesis project that is in progress as this is being written is investigating the supersonic loss behavior. In any case, it is evident that the supersonic correction applied in the KO system does not allow for such loss plateaus. 


\subsection{Effects of Reynolds Number and Surface Roughness on Profile Loss Predictions}

\subsubsection{Introduction}

As shown in Chapter 2, the total pressure loss coefficient $Y$ in the Kacker \& Okapuu loss system is

$$
Y=Y_{P} \times f(R e)_{K O}+Y_{s}+Y_{T E T}^{\prime}+Y_{T C}
$$

$f(R e)_{K O}$ in Equation 5.6 is a Reynolds number correction factor applied to the profile loss predictions. The Ainley \& Mathieson data consists mostly of cascade data with a Reynolds number of 200,000. Therefore, in order to use the loss data from AMDC to predict the losses of a turbine cascade with a significantly different Reynolds number, the KO profile losses must be corrected.

Such a correction has not been applied to the loss predictions presented in the previous section. The Reynolds number for each cascade generally falls within the range over which $f(R e)_{K O}$ is, or is close to, 1.0. However, at the highest Reynolds numbers (above $1 \times 10^{6}$ ), and for the midspan section E2S50, which is not hydraulically smooth, a correction factor should be applied to the predicted losses. Unfortunately, the KO loss system has no means by which to account for surface roughness. Aungier (2006) offers an alternative Reynolds correction factor, $f(R e)_{\text {Aungier }}$, which accounts for surface roughness at sufficiently high Reynolds numbers. The following sections will describe the Reynolds correction of Aungier. For comparison,

both $f(R e)_{K O}$ and $f(R e)_{\text {Aungier }}$ will be used to correct the predicted losses of E2S50. This cascade not only has some surface roughness, but also has a Reynolds number above $1 \times 10^{6}$ at outlet Mach numbers above about 1.0.

\subsubsection{Alternatives to the Kacker \& Okapuu Reynolds Number Correction and the Effects of Surface Roughness}

Ainley \& Mathieson (1951) built their loss prediction system upon cascade data which 
primarily had a Reynolds number of 200,000. They suggested that to estimate the performance of turbines with Reynolds numbers below 200,000, a correction factor should be applied. This was done in the form of a correction to the predicted efficiencies as follows:

$$
1-\eta_{\text {corrected }}=(1-\eta)\left(\frac{R e}{2 \times 10^{5}}\right)^{-0.2}
$$

Dunham \& Came $(1970)$ revised this by applying the Reynolds correction of $\left(\frac{R e}{2 \times 10^{5}}\right)^{-0.2}$ to the predicted profile losses from Ainley \& Mathieson, rather than to the efficiency. As shown in Equation 5.8, Kacker \& Okapuu (1982) (KO) further modified the Reynolds number correction from the Ainley \& Mathieson and Dunham \& Came loss system (AMDC) in the low Reynolds number range, and also added a high Reynolds number correction factor. There is no correction over the range $2 \times 10^{5}<R e_{c}<1 \times 10^{6}$ which is assumed to be the transitional range for the boundary layers. The effect of transition on the losses was not well documented at the time of KO's work and therefore they did not propose a correction.

$$
f(R e)_{K O}= \begin{cases}\left(\frac{R e_{c}}{2 \times 10^{5}}\right)^{-0.4} & \text { for } R e_{c} \leq 2 \times 10^{5} \\ 1.0 & \text { for } 2 \times 10^{5}<R e_{c}<1 \times 10^{6} \\ \left(\frac{R e_{c}}{1 \times 10^{6}}\right)^{-0.2} & \text { for } R e_{c} \geq 1 \times 10^{6}\end{cases}
$$

In this form, the Kacker \& Okapuu Reynolds number correction is similar to that of Denton (1973). However, while Denton accounts for surface roughness, Kacker \& Okapuu did not. More recently, several alternative Reynolds number correction factors have been proposed by different authors; for example, from Carleton, Zhu \& Sjolander (2005) and Hall (2012) have proposed alternative Reynolds number correction factors for the low and mid Reynolds number ranges, respectively. Like Kacker \& Okapuu these do not account for surface roughness and will not be discussed in this thesis. Aungier (2006) offers an alternative to the Kacker \& Okapuu Reynolds correction that does account for surface roughness.

The Reynolds number correction factor due to Aungier $f(R e)_{\text {Aungier }}$ is very similar in form to that due to Kacker \& Okapuu. Each is a piece-wise function of Reynolds number, with three distinct sections. Each has a low Reynolds number correction, a region of no correction, and a high Reynolds number correction. $f(R e)_{\text {Aungier }}$ differs from the $f(R e)_{K O}$ in 
the high Reynolds number range in that it takes into account the effects of surface roughness. Roughness is only considered when the Reynolds number is greater than 500,000. Aungier characterizes the roughness using the average height of the roughness elements, $e$.

Aungier s correction for smooth airfoils $(e=0)$ is

$$
f(R e)_{\text {Aungier }_{e=0}}= \begin{cases}\left(\frac{R e_{c}}{10^{5}}\right)^{-0.5} & \text { for } R e_{c} \leq 10^{5} \\ 1.0 & \text { for } 10^{5}<R e_{c}<5 \times 10^{5} \\ \left(\frac{\log _{10}\left(R e_{c}\right)}{\log _{10}\left(5 \times 10^{5}\right)}\right)^{-2.58} & \text { for } R e_{c} \geq 5 \times 10^{5}\end{cases}
$$

This is similar to the correction due to Kacker \& Okapuu and Denton. However, Aungier's correction factor uses a different reference Reynolds number. Since Kacker \& Okapuu were building on the work of Ainley \& Mathieson and Dunham \& Came, they continued to use 200,000 as the reference Reynolds number for the low Reynolds number correction factor. In the $\mathrm{KO}$ correction, the completion of the transitional range is at a Reynolds number of $1 \times 10^{6}$

The reference Reynolds numbers used by Aungier, namely 100,000 and 500,000, were also chosen to represent the boundaries of the three flow regimes: laminar, transitional and turbulent. Of course, these are approximations since transition does not necessarily begin exactly at $R e_{c}=10^{5}$ for all turbines, and there is likely always some region of laminar flow present on the blade surface, even at high outlet Reynolds number.

In the case of rough-surface turbines, Aungier's Reynolds number correction shows the greatest differences from Kacker \& Okapuu. Above Reynolds numbers of 500,000, surface roughness affect loss production by causing earlier onset of transition and by increasing skin friction in the turbulent boundary layer (Denton, 1973$)$. The skin friction coefficient becomes independent of Reynolds number when the roughness elements, of height e, penetrate beyond the laminar sublayer, which is thought to occur when $R e_{c}>100 c / e$ (Schlichting, 1962; Denton, 1973; Aungier, 2006). Aungier calls this the critical roughness Reynolds number, $R e_{r}$, above which the correction factor is independent of Reynolds number. For turbines with a critical Reynolds number less than 500,000 and blade Reynolds numbers above 500,000 (i.e.: $R e_{r}<$ $\left.500,000<R e_{c}\right)$, only the portion of the boundary layer which is turbulent is influenced by the roughness; the correction factor uses a weighted average based on outlet Reynolds number 
to account for this. Aungier's correction for rough turbines is

$$
f(R e)_{\text {Aungier }}= \begin{cases}\left(\frac{R e_{c}}{10^{5}}\right)^{-0.5} & \text { for } R e_{c} \leq 10^{5} \\ 1.0 & \text { for } 10^{5}<R e_{c}<5 \times 10^{5} \\ \left(\frac{\log _{10}\left(R e_{c}\right)}{\log _{10}\left(5 \times 10^{5}\right)}\right)^{-2.58} & \text { for } 5 \times 10^{5}<R e_{c}<R e_{r} \\ \left(\frac{\log _{10}\left(R e_{r}\right)}{\log _{10}\left(5 \times 10^{5}\right)}\right)^{-2.58} & \text { for } 5 \times 10^{5}<R e_{r}<R e_{c} \\ 1+\left[\left(\frac{\log _{10}\left(R e_{r}\right)}{\log _{10}\left(5 \times 10^{5}\right)}\right)^{-2.58}-1\right]\left(1-\frac{5 \times 10^{5}}{R e_{c}}\right) & \text { for } R e_{r}<5 \times 10^{5}<R e_{c}\end{cases}
$$

Both the Kacker \& Okapuu and Aungier correction factors are plotted in Figure 5.15. Indicated on the figure is the Reynolds number range covered by the Siemens cascade results in the present study. The Siemens cascades fall in the range of Reynolds numbers where KO and Aungier show noticable disagreement, even for smooth airfoils.

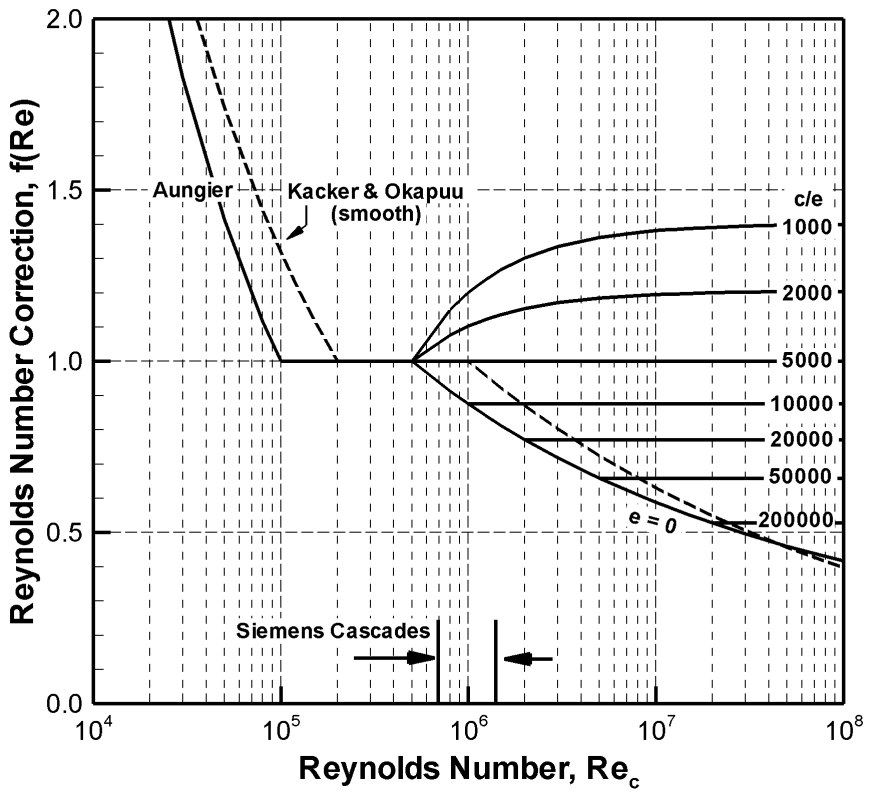

Figure 5.15: Reynolds Corrections for Rough Cascades due to Kacker \& Okapuu (1982) and Aungier (2006)

Excluding the transitional Reynolds number range, over which $f(R e)$ is constant, the 
correction factor, and hence the predicted losses, decreases with increasing Reynolds number for smooth turbines; this is true for both Kacker \& Okapuu and Aungier. When roughness is considered, the losses decrease with increasing Reynolds number so long as $R e_{c}<R e_{r}$, after which the correction factor remains constant.

Standard turbine surface finish is usually between $c / e=10,000$ and 20,000 Koch \& Smith, 1976). Therefore, in general practice, roughness will only effect turbines with Reynolds numbers higher than about $1 \times 10^{6}$. For cascade testing on the other hand, the blades should ideally be hydraulically smooth to avoid any roughness effects; this is especially important for high-speed cascade testing, like that which is discussed in this thesis, where Reynolds numbers are high. As mentioned in previous chapters, the laser-sintering manufacturing method of the midspan cascade E2S50 did not yield a hydraulically smooth blade.

In the following section, the loss predictions for E2S50 will be corrected for Reynolds number effects using both the Reynolds corrections of $\mathrm{KO}$ and Aungier.

\subsubsection{Effect of Reynolds Correction on E2S50 Loss Prediction}

The previous section described an alternative to the Kacker \& Okapuu Reynolds correction factor $f(R e)_{K O}$. This alternative was due to Aungier (2006). This section will compare the two, and evaluate the accuracy with which they predict the losses for the rough midspan cascade E2S50. This is a very limited comparison. Further evaluation of the Reynolds correction due to Aungier, $f(R e)_{\text {aungier }}$, is required before it can be recommended as a replacement for $f(R e)_{K O}$.

Aungier]s correction for Reynolds number requires knowledge of the chord-to-roughness height ratio, $c / e$. Since the roughness of E2S50 was not an intended design feature, but rather an artifact of the manufacturing process, the roughness had to be measured. This was done by Siemens and it was found that the chord-to-roughness height ratio was about $c / e=2000$ for this cascade.

Figure 5.16 shows the predicted loss for E2S50 using the standard KO Reynolds correction

as well as Aungier's correction. Two roughness values were used to calculate $f(R e)_{\text {aungier }}$; the estimated actual roughness (i.e: $c / e=2000$ ), and no roughness (i.e.: $e=0$ ). In order 


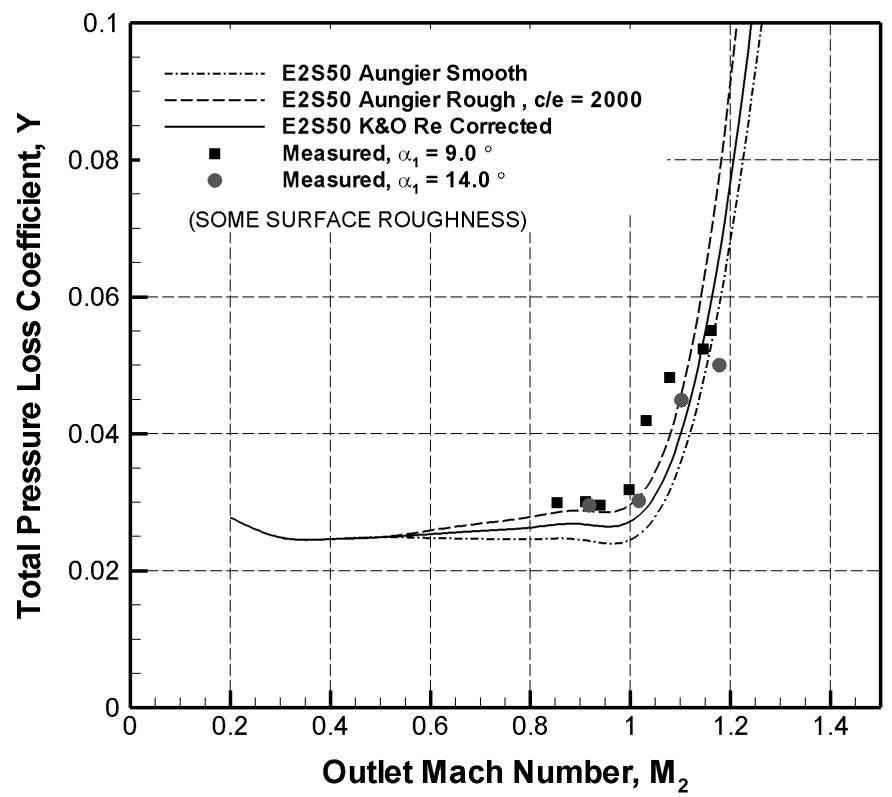

Figure 5.16: Predicted loss for E2S50: Effect of Reynolds Correction

to correct for Reynolds number over a wide range of Mach numbers, as is done here, the relationship between Mach and Reynolds numbers must be known. The experimental data for Reynolds number, shown in Section 5.5.3, is used to establish this relationship. Using an exponential fit, the relationship between Mach number and Reynolds number is

$$
R e_{c}=239346 e^{1.4639 \times M_{2}}
$$

where $e$ is Euler's constant and not roughness in this equation. The coefficient of determination for this fit is $R^{2}=0.9876$.

Figure 5.16 shows that at lower Mach numbers (below about $M_{2}=0.5$ ), there is no difference between the two corrections, since Reynolds number is below 500,000 for these cases. When compared to the original predictions shown in Section 5.6, the KO Reynolds correction had little effect. Only above an outlet Mach number of about 1.0 did the predictions change. Therefore, the uncorrected predictions are not repeated on this figure.

The effects of roughness on the loss predictions are significant above about $M_{2}=0.6$. Aungier's correction, which includes roughness, shows the best match to the experiments, 
specifically between $M_{2}=0.8$ and 1.0. Including $f(R e)_{\text {Aungier }}$ for rough cascades in the predictions seems to have made up for the underprediction of the subsonic losses shown in Section 5.6. However, the correction for smooth cascades by Aungier increases the amount of underprediction when compared with the KO correction.

\subsection{Future Work}

\subsubsection{Introduction}

Previous sections in this chapter have discussed the comparisons between the experimentally measured losses of the three cascades with the predicted losses from the Kacker \& Okapuu (KO) loss system. As seen, the comparisons have shed light on several shortcomings of the KO loss system. This section expands on these shortcomings and offers suggestions for further research to resolve the issues.

\subsubsection{Effect of Negative Inlet Angles on Kacker \& Okapuu Profile Loss Prediction}

As shown in Chapter 2, the work of Ainley \& Mathieson (1951) and Dunham \& Came (1970) (referred to collectively as AMDC), which forms the basis of the Kacker \& Okapuu (1982) loss system, is fundamentally based on the experimental loss data of two special turbine geometries: nozzle blades, which have axial in-flow (i.e.: $\frac{\beta_{1}}{\alpha_{2}}=0$ ), and impulse blades, for which inlet and outlet flow angles are the same (i.e.: $\frac{\beta_{1}}{\alpha_{2}}=1.0$ ). Loss data were collected over a range of outlet flow angles $\alpha_{2}$ and pitch-to-chord ratios $s / c$ for both nozzles and impulse blades. The AMDC loss system was designed to predict the losses of geometries that fall between these two cases.

The AMDC loss system was also intended for use at the meanline. At that location, the inlet flow or metal angle is not normally negative. Therefore, the AMDC system did not account for that possibility. However, for lightly loaded turbine stages the inlet angle can occasionally be negative even at the meanline. Furthermore, the meanline correlations have subsequently been used to predict the profile losses across the full blade span in throughflow 
analyses. Towards the blade tip, turbine rotor blades can have significantly negative values of inlet angle. Thus, the geometry is well outside the range for which AMDC was intended. As a result, the AMDC system gives implausibly high predictions for the profile losses at negative values of the inlet angle. This can be seen as follows.

As described in Chapter 2, the original expression due to Ainley \& Mathieson for the basic weighted profile loss for an arbitrary turbine blade geometry is

$$
Y_{P, A M}=\left[Y_{P N}+\left(\frac{\beta_{1}}{\alpha_{2}}\right)\left(\frac{\beta_{1}}{\alpha_{2}}\right)\left(Y_{P I}-Y_{P N}\right)\right]\left(\frac{t_{\max } / c}{0.2}\right)^{\frac{\beta_{1}}{\alpha_{2}}}
$$

where $Y_{P N}$ and $Y_{P I}$ are the loss coefficients for the nozzle and impulse blades having the same outlet flow angle and pitch-to-chord ratio as the blade of interest. The cascade data of Ainley \& Mathieson showed that the impulse blade always has higher losses than the nozzle (i.e.: $\left.Y_{P I}>Y_{P N}\right)$ and the losses for all other geometries fall between these two extremes. Consider then the case of $\beta_{1}=-\alpha_{2}$, which corresponds to a blade with zero turning. Equation 5.12 predicts that this blade will have $Y_{P}=Y_{P I}$ : that is, the losses will be the same as those for the geometry that produces the highest possible losses, namely the impulse blade. Given that the zero-turning blade is not generating any lift, it would be expected to have very low losses and the AMDC prediction is clearly unreasonable.

Kacker \& Okapuu (1982) recognized this problem and modified Equation 5.12 to

$$
Y_{P, A M D C}=\left[Y_{P N}+\left|\frac{\beta_{1}}{\alpha_{2}}\right|\left(\frac{\beta_{1}}{\alpha_{2}}\right)\left(Y_{P I}-Y_{P N}\right)\right]\left(\frac{t_{\max } / c}{0.2}\right)^{\frac{\beta_{1}}{\alpha_{2}}}
$$

The subscript "AMDC" is used to denote this form of the profile loss expression to maintain consistency with Kacker \& Okapuu (1982). Equation 5.13 predicts that losses decrease as $\beta_{1}$ becomes increasingly negative. The generic trends in losses with $\beta_{1} / \alpha_{2}$ as predicted by both AMDC and KO are shown in Figure 5.17 for a family of geometries with fixed $\alpha_{2}$ and $s / c$.

As implied by Figure 5.17, the KO expression can predict negative values of the loss for sufficiently large negative values of $\beta_{1}$. It can be shown from Equation 5.13 that this is possible if $\frac{Y_{P I}}{Y_{P N}}>2.0$. The values of this ratio as given by the AMDC correlations were therefore examined. As shown in Figure 5.18, $Y_{P I} / Y_{P N}$ is in fact greater than 2.0 for the full range of $s / c$ and $\alpha_{2}$ covered by the AMDC profile loss correlation. Consequently, the KO 


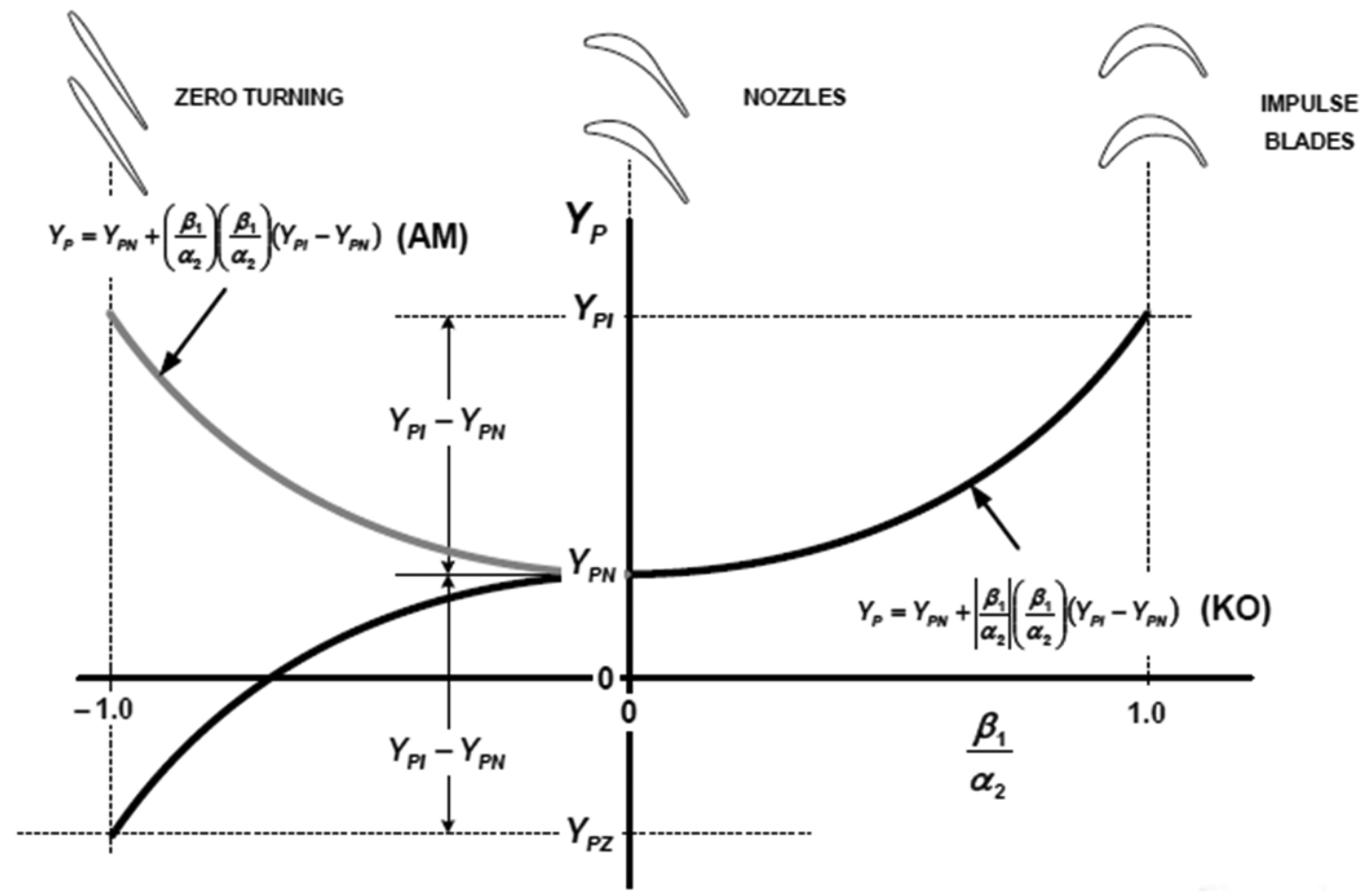

Figure 5.17: Loss Prediction at Negative Inlet Angles

predictions, which also use the AMDC values of $Y_{P I}$ and $Y_{P N}$, can also result in negative losses for sufficiently large negative $\beta_{1}$.

Figure 5.19 plots the losses that are predicted by the KO expression for the families of turbine geometries of which the four Siemens cascades are members. The figures indicate the specific values $\beta_{1} / \alpha_{2}$ that apply to the Siemens geometries. As seen, Kacker \& Okapuu predicts positive profile losses for all four cases. However, the low-turning tip section, E1S90, for which $\frac{\beta_{1}}{\alpha_{2}}=-0.48$, is just short of the value for which the $\mathrm{KO}$ expression begins to predict negative losses. This explains the unexpectedly low profile loss predictions noted for this airfoil in Figure 5.14(b),

The ratio $\beta_{1} / \alpha_{2}$ also appears as an exponent on the thickness correction in both the original AMDC and the KO expressions for basic weighted profile losses (Equations 5.12 and 5.13). The thickness correction, $K_{t}$, is given by

$$
K_{t}=\left(\frac{t_{\max } / c}{0.2}\right)^{\frac{\beta_{1}}{\alpha_{2}}}
$$




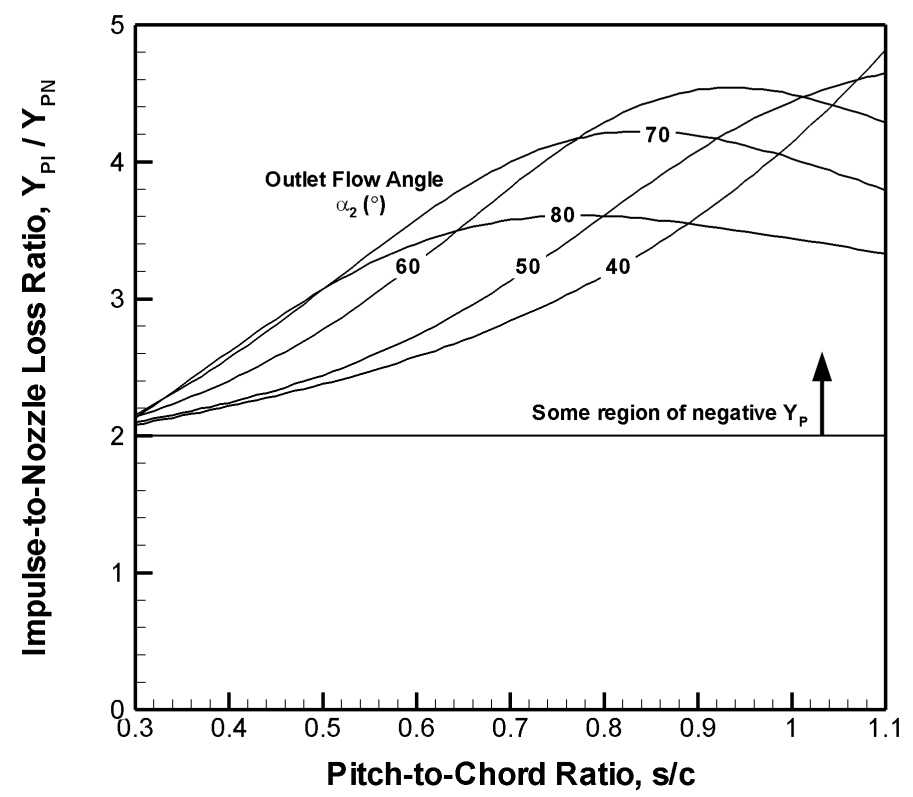

Figure 5.18: Ratio of $Y_{P I}$ to $Y_{P N}$ from AMDC Correlation

where $t_{\max }$ is the maximum thickness of the blade.

The Ainley \& Mathieson loss data were obtained for turbines which mostly had a maximum blade thickness $20 \%$ of the chord length; that is, $\frac{t_{\max }}{c}=0.2$. The thickness correction is included to account for geometries with a different maximum blade thickness. The correction was intended to behave in such a way that the losses increase when the $\frac{t_{\max }}{c}>0.2$ and decrease losses when the opposite is true. As shown in Figure 5.20, the correction does not function as it should for all possible values of $\beta_{1} / \alpha_{2}$. For geometries with negative inlet angles, the thickness correction acts to decrease predicted losses with increasing thickness.

Interestingly, the ratio $t_{\max } / c$ for the two Siemens tip sections with negative inlet angles, E1S90 and E2S90, is less than 0.2. Thus, despite being very small to begin with, the predicted losses shown in Section 5.6 for these two cascades have actually been inflated due the thickness correction.

In summary, while still an improvement, the Kacker \& Okapuu modifications to the Ainley \& Mathieson loss system to allow for negative values of inlet angle $\beta_{1}$, is clearly not satisfactory. It leads to unrealistically low and even negative values of profile losses for turbine blade 


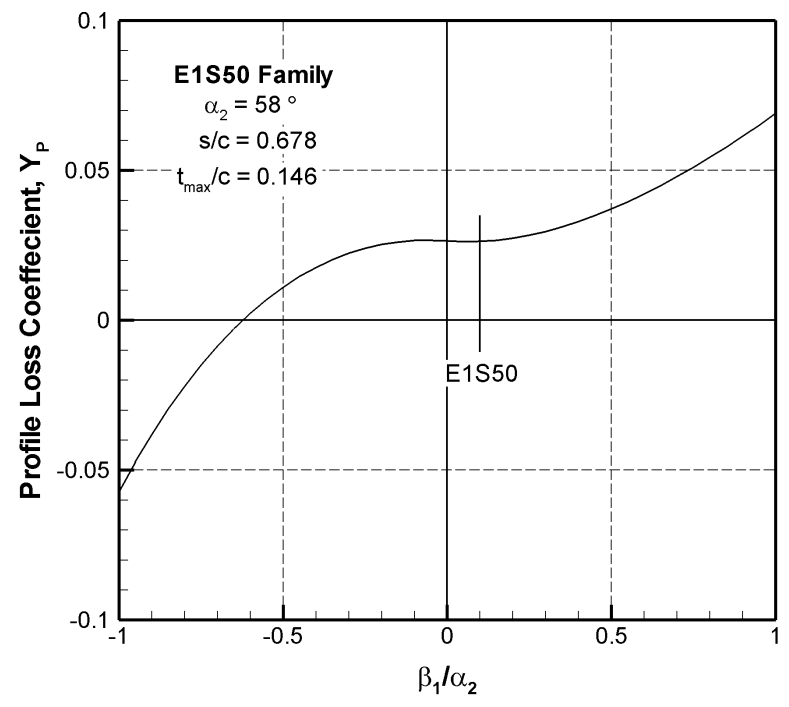

(a)

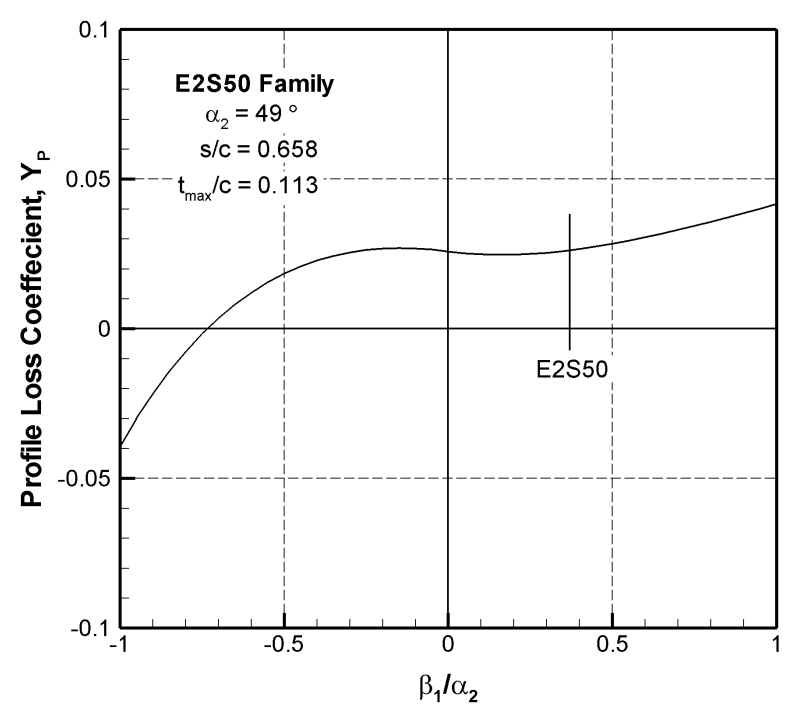

(c)

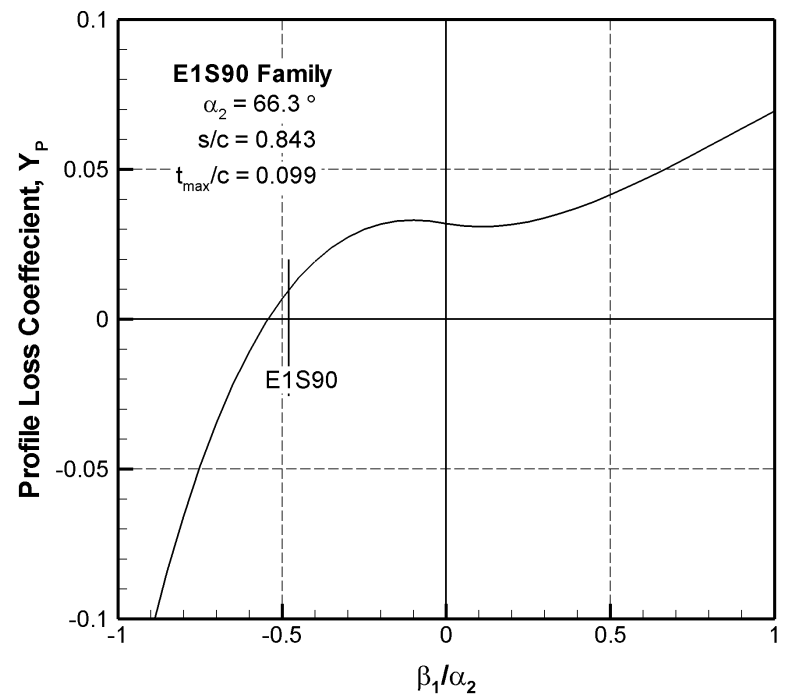

(b)

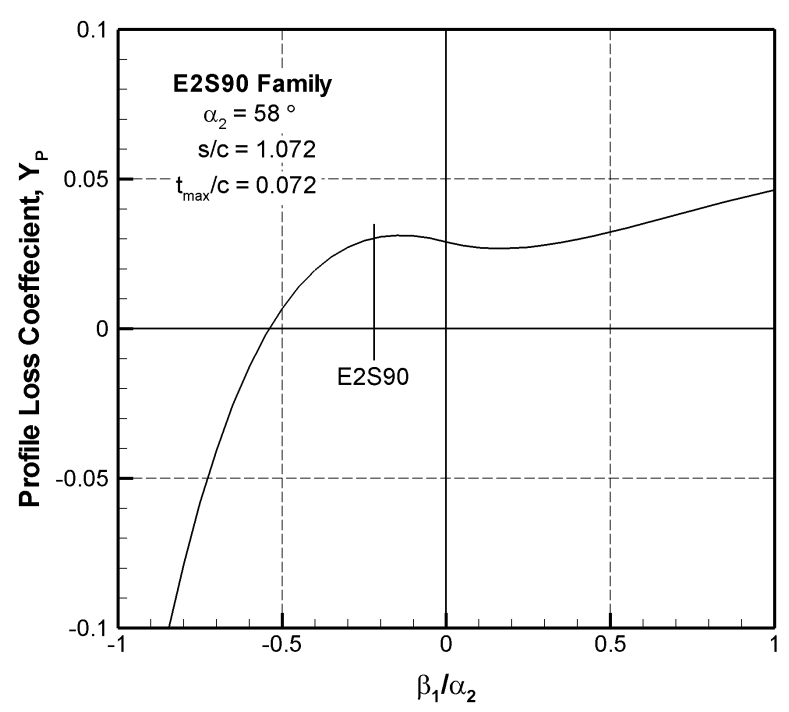

(d)

Figure 5.19: Kacker \& Okapuu Predicted Profile Losses for Siemens Cascade Families 


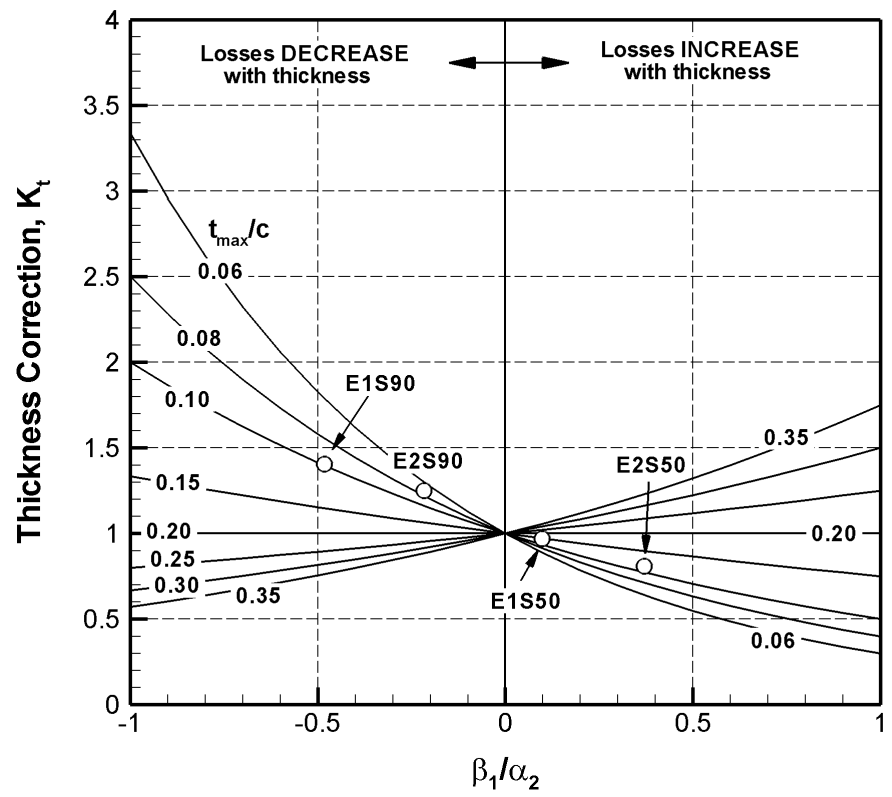

Figure 5.20: Effect of $\frac{\beta_{1}}{\alpha_{2}}$ on $K_{t}$

geometries that can occur in practice. The underprediction seems to arise from the expression for the Kacker \& Okapuu basic weighted profile losses (Equation 5.13).

Further research is required to remedy these issues. Future studies should focus on expanding the available data for cascades with large negative inlet angles. Following the form of AMDC, a third body of data might be collected and added to the correlated data for impulse blades and nozzle blades. The third set could contain profile loss measurements for zero-turning cascades at the same range of $s / c$ and $\alpha_{2}$ as the AMDC data. Equation 5.13 might then be modified such that, for geometries with negative inlet angles, the zero-turning loss coefficients $Y_{P Z}$ would be used for the interpolation as follows

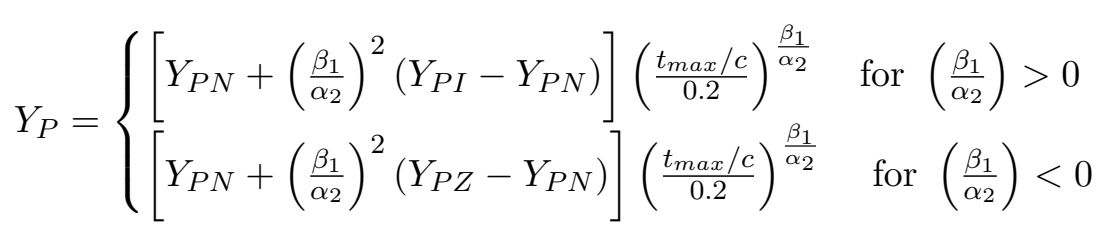




\subsubsection{Effect of Negative Inlet Angles on Kacker \& Okapuu Trailing Edge Loss Prediction}

As mentioned earlier, the subsonic losses for the Siemens cascades have been underpredicted by the KO loss system. The midspan losses predicted by the KO system are the sum of two contributions: the profile losses generated in the blade surface boundary layers, and the trailing edge losses due to the base pressure and sudden expansion in flow area at the trailing edge. The previous section highlights shortcomings of the KO system for predicting profile losses. This section will examine the trailing edge loss component to the KO loss system.

From Figure 5.14, it was shown that the trailing edge loss predictions for all the Siemens cascades are very small. This can be attributed to the very thin trailing edges of these cascades. However, it is not possible to distinguish between the two sources of loss generation experimentally. Therefore, its not possible to conclude whether the overall loss underprediction shown is caused by the issues with the profile loss prediction described in the previous section or by a significant underprediction of the trailing edge losses. Mee et al. (1992) have

shown that the trailing edge loss can contribute up to $\frac{1}{3}$ of the total two-dimensional midspan loss for subsonic cascades and as much as half the loss for supersonic cascades.

The trailing edge loss component of the KO loss system follows a similar form to the profile loss component. The trailing edge loss coefficient is calculated as a weighted average between the trailing edge losses of nozzles and impulse blades with the same outlet flow angle and pitch-to-chord ratio as the blade of interest. For trailing edge losses, the energy loss coefficient $\Delta \phi_{T E T}^{2}$, not the total pressure loss coefficient $Y$, is used. The resulting expression for trailing edge energy loss coefficient is then

$$
\Delta \phi_{T E T}^{2}=\Delta \phi_{T E T\left(\beta_{1}=0\right)}^{2}+\left|\frac{\beta_{1}}{\alpha_{2}}\right|\left(\frac{\beta_{1}}{\alpha_{2}}\right)\left(\Delta \phi_{T E T\left(\beta_{1}=\alpha_{2}\right)}^{2}-\Delta \phi_{T E T\left(\beta_{1}=0\right)}^{2}\right)
$$

Kacker \& Okapuu provide the following conversion between energy loss and total pressure loss coefficients:

$$
Y_{T E T}^{\prime}=\frac{\left[1-\frac{\gamma-1}{2} M_{2}^{2}\left(\frac{1}{1-\Delta \phi_{T E T}^{2}}-1\right)\right]^{-\frac{\gamma}{\gamma-1}}-1}{1-\left(1+\frac{\gamma-1}{2} M_{2}^{2}\right)^{-\frac{\gamma}{\gamma-1}}}
$$




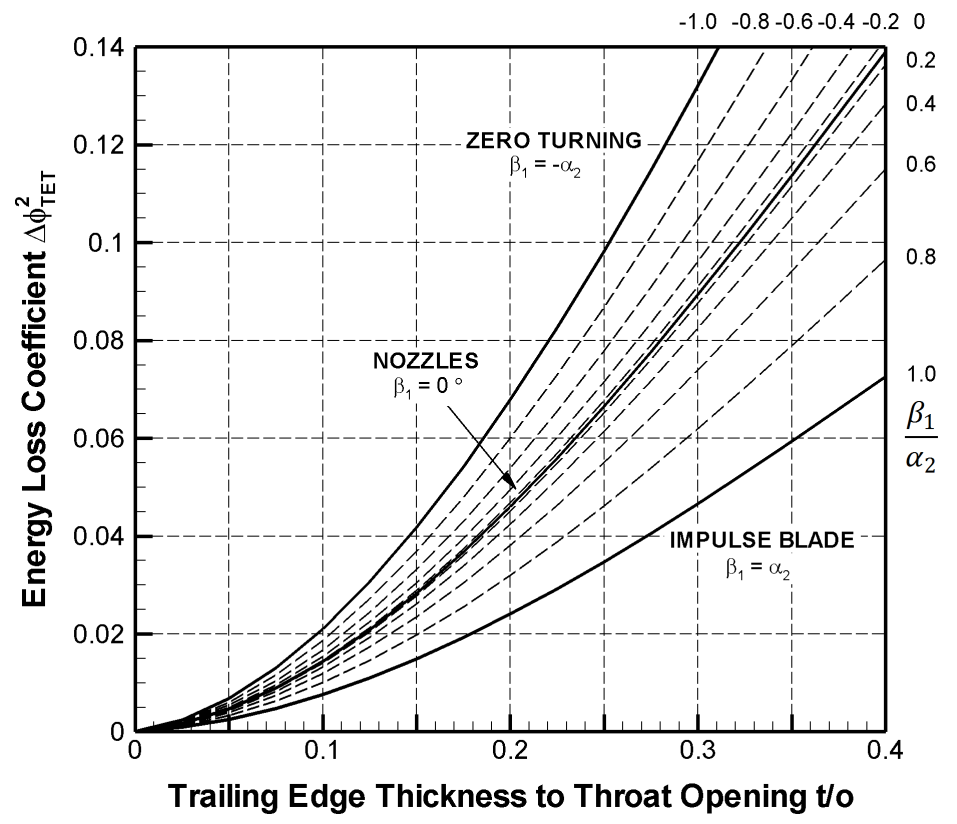

Figure 5.21: Effect of $\frac{\beta_{1}}{\alpha_{2}}$ on $\Delta \phi_{T E T}^{2}$

Figure 5.21 shows the $\mathrm{KO}$ predictions for the trailing edge loss coefficient $\Delta \phi_{T E T}^{2}$ as a function of trailing edge blockage $t / o$ for nozzles and impulse blades. Equation 5.16 was used to plot the behaviour of $\Delta \phi_{T E T}^{2}$ for several values of $\beta_{1} / \alpha_{2}$ varying between 1.0 (impulse blades) and -1.0 (zero turning cascades). The KO data shows that the impulse blade always has lower losses than nozzles (i.e.: $\left.\Delta \phi_{T E T\left(\beta_{1}=0\right)}^{2}>\Delta \phi_{T E T\left(\beta_{1}=\alpha_{2}\right)}^{2}\right)$. This can be explained by considering the approximate expression for trailing edge loss coefficient due to Denton (1993):

$$
Y_{t e}=\frac{\Delta P_{0}}{\frac{1}{2} \rho V_{2}^{2}}=-\frac{C_{P_{b}} t}{o}+\frac{2 \theta}{o}+\left(\frac{\delta^{*}+t}{o}\right)^{2}
$$

The first term in Equation 5.18 represents the loss due to base pressure acting on the trailing edge. Prior to the work of Mee et al. (1992) and Denton (1993), base pressure effects were widely neglected resulting in an underprediction of trailing edge losses. The second term accounts for the entropy rise caused by the downstream mixing of the two blade surface boundary layers. The third and final term in Equation 5.18 accounts for the loss generated by the sudden expansion in flow area at the trailing edge, including the contribution to the 
effective area change due to the displacement effect of the boundary layers.

For a fixed trailing edge blockage $t / o$, the predicted energy loss coefficient increases with decreasing $\beta_{1} / \alpha_{2}$. The dependency of the trailing edge loss on $\beta_{1} / \alpha_{2}$ is attributed to the change in passage acceleration, or more specifically the change in the thickness of the boundary layer at the trailing edge. Highly accelerated cascades, such as nozzles, tend to have thinner blade surface boundary layers at the trailing edge. On the other hand, impulse blades have no net acceleration from inlet to outlet. Consequently, any local acceleration that takes place on the blade will be countered by an equal amount of deceleration, which is likely to thicken the boundary layer. Kacker \& Okapuu suggest that thinner boundary layers result in larger (more negative) base pressure coefficients. Denton \& Xu (1990) attribute the decrease in base pressure to the lower momentum thickness of thin boundary layers. In boundary layers with low momentum thickness there is higher momentum near the separated shear layer which can entrain base region fluid, decreasing the base pressure and thus increasing loss. For a given $t / o$, nozzles will always have higher passage acceleration, thus thinner boundary layers. Consequently, nozzles will always produce higher trailing edge losses.

Figure 5.21 shows that losses increase with decreasing $\beta_{1} / \alpha_{2}$. This is expected for the range between 1.0 and 0 where the decrease in $\beta_{1} / \alpha_{2}$ leads to an increase in acceleration and hence thinner boundary layers. However, one would expect the trend to reverse when $\frac{\beta_{1}}{\alpha_{2}}<0$ where the passage acceleration decreases as the geometry approaches a zero-turning cascade. However, this is not what the correlation gives: the losses continue to increase with decreasing $\beta_{1} / \alpha_{2}$.

Similar to the profile loss predictions, the KO predictions for the trailing edge losses are unsatisfactory at negative inlet angles. To address this issue, future studies need a means by which to separate trailing edge losses from other sources of midspan losses such as shocks and boundary layer losses. Mee et al. (1992) made detailed measurements of the boundary layers near the trailing edge to determine the profile losses, and examined the mid-passage losses downstream of the cascade to determine the shock losses. The trailing edge losses can then be estimated by subtracting the profile losses and shock losses from the total midspan losses. 


\subsubsection{Mach Number and Reynolds Number Corrections}

The AMDC data were collected at low subsonic Mach numbers and at an average Reynolds number of 200,000. Losses, however, are not independent of Mach number or Reynolds number. As such, Kacker \& Okapuu include several correction factors in the loss prediction system. These include Reynolds number correction $f(R e)_{K O}$ discussed in Section 5.7.2, a subsonic compressibility correction $k_{p}$ and a supersonic correction $k_{c}$. All the corrections are applied to the predicted profile losses as follows:

$$
Y_{p}=f(R e)_{K O}\left[0.914\left(\frac{2}{3} Y_{P, A M D C}\right)\right] \times k_{p} \times k_{c}
$$

Kacker \& Okapuu loss predictions all show a steep and steady rise in loss above Mach 1.0. Contrary to this, some experimental results show a tendency for the supersonic loss to level off in a plateau for some range of Mach numbers above Mach 1.0. This suggests that there are more factors to consider than just the Mach number when calculating the supersonic Mach number correction.

The supersonic Mach number correction, $k_{c}$, is given in Equation 2.16, and is a function of Mach number only. Kacker \& Okapuu (1982) suggest that outlet angle should also be considered to have an effect on the supersonic Mach number correction, to account for airfoil limit loading. Other authors such as Ainley \& Mathieson (1951), Craig \& Cox (1971), Graham \& Kost (1979), and Sieverding (1985) mention the influence of suction side curvature downstream of the throat and unguided turning, $\theta_{U G}$, on supersonic loss.

Experimental results presented in Figure 5.14 show some evidence of a loss plateau at low supersonic Mach numbers, contrasting the Kacker \& Okapuu prediction of a steady monotonic rise. E1S50 $\left(\theta_{U G}=4.6^{\circ}\right)$ appears to have an early onset and long lasting supersonic loss plateau, whereas E2S50 and E2S90 $\left(\theta_{U G}=9.1^{\circ}\right.$ and $13.7^{\circ}$ respectively $)$ only hint at the presence of a plateau starting at the highest measured Mach numbers. Further experiments at higher Mach numbers are required to investigate and understand these phenomena. Future studies should preferably also include a number of cascades which have different levels of unguided turning to assess the effect it may have on supersonic loss. As mentioned earlier, there is currently a thesis project at Carleton studying the supersonic loss behavior of several 
turbine cascade geometries.

Kacker \& Okapuu also predicts a slight drop in loss just before Mach 1.0 but fails to accurately predict the onset and magnitude of this phenomenon for the tip section E2S90. The Kacker \& Okapuu subsonic correction is a function of the outlet Mach number and passage acceleration. Section 5.7 .3 shows that Reynolds number can also have an effect on subsonic loss. Hall (2012) investigated the effects of Reynolds number and Mach number on subsonic loss estimation. Hall suggests that these parameters effect loss production by influencing the transition location on the blades. Transition location is not often known early in design and is not a likely candidate for inclusion in a correction parameter. Future studies should investigate the relationship between Reynolds number and Mach number and the combined effect they have on subsonic loss production. Additionally, Kacker \& Okapuu apply no correction to the trailing edge losses for the effects of Reynolds number or Mach number. $Y_{T E T}^{\prime}$ is dependent on the state of the blade surface boundary layers at the trailing edge, which is influenced by $R e_{c}$ and $M_{2}$, and thus it is likely that $Y_{T E T}^{\prime}$ will also be influence by these parameters.

This thesis points to a number of potential studies that could further improve loss estimation for the early stages in design. Many of these suggested studies require substantial amounts of data which can be time consuming and costly to acquire. CFD is a possible alternative to experiment since many different turbine geometries and operating conditions can be tested with relative ease. CFD also allows for the independent study of Reynolds and Mach number effects, which can be difficult to do experimentally. 


\section{Chapter 6}

\section{Conclusions and Recommendations for Future Work}

\subsection{Summary}

Meanline analysis in the preliminary design of modern turbines relies on the ability to accurately predict the losses of the turbine being designed. Loss prediction is typically based on empirical cascade data obtained over the past five or six decades. As the design technologies improve and the application of meanline analysis extends away from the mean radius of the blade, the loss correlations must be updated and improved with additional empirical data.

In the current study, cascade measurements of the midspan flows were obtained for three turbines from two Siemens industrial engines: the two midspan sections designated E1S50 and E2S50, and the tip section designated E2S90. The measurements were obtained at several transonic outlet Mach numbers and inlet flow angles. The outlet Mach numbers ranged from 0.8 to as high as 1.4. The inlet angles for the three cascades varied widely from $-30^{\circ}$ to $30^{\circ}$. The loss data obtained at all inlet flow angles were analysed to estimate the design value of inlet flow angle, which is unknown for the Siemens cascades. The loss measurements presented in this study provide a contribution to the database on the performance of transonic turbine blades available in open literature. Furthermore, certain design parameters of the turbines, such as inlet metal angle, lie near to, and in some cases, just beyond the recommended limits of current loss prediction systems. Hence, these data can help to develop improvements to certain aspects of the loss correlations.

For all cascades, data collected at the two values of inlet angle estimated to be nearest to 
the design value were taken as an approximation of the design incidence losses. It was shown that for all cascades there was not a large dependency on inlet angle near the estimated design incidence.

The influence of outlet Mach number on losses and outlet flow angle was examined for all three cascades near the estimated design point. Losses and flow parameters were calculated by mixing wake traverse data following the Amecke \& Šafařík (1995) flow mixing procedure. The results of the loss measurements were then compared with the predictions from the Kacker \& Okapuu (1982) loss prediction system for design incidence.

\subsection{Conclusions}

It was found that the Kacker \& Okapuu loss system did not accurately predict the losses for all cascades. Several shortcomings were identified: the supersonic plateau in losses observed experimentally for the midspan cascade E1S90 was not predicted by the Kacker \& Okapuu loss system, the magnitude of the dip in losses predicted just below $M_{2}=1.0$ did not match the experimental measurements, and the predicted losses for the rough midspan cascade did not account for the surface roughness. Additionally, the tip section cascade E1S90, for which the inlet angle is $-32^{\circ}$, was shown to have unreasonably low loss predictions: significantly less than half the values for the other three cascades. The low predicted losses are believed to be a result of both the underprediction of the trailing edge losses as well as the profile losses. This is thought to be partly related to the way in which negative inlet angles are treated in the Kacker \& Okapuu loss system.

As described in Chapter 5, profile losses for turbines with large negative inlet angles are

predicted to be low and in some cases the possibility for the predicted losses to be negative exists. This is a result of the way in which the losses are predicted by a weighted interpolation between the empirical data for nozzles and impulse blades which have the same solidity and outlet flow angle as the blade for which the prediction is being made. This approach is perfectly adequate for turbines whose inlet flow angle falls between the case of the nozzle and impulse blade. The predicted losses are maximum for impulse blades and decrease as the 
inlet metal angle approaches that of the nozzle. However, it was shown that the predicted losses continue to decrease as the inlet angle is reduced to negative values. Furthermore, due to the relative magnitudes of the nozzle and impulse blade losses, the losses of turbines with sufficiently large values of negative inlet angle will be predicted to be negative. In addition to the profile losses, the trailing edge loss predictions from the Kacker \& Okapuu are unsatisfactory for turbines with negative inlet angles. The inlet angle for cascade E1S90 is very close to the value for which the Kacker \& Okapuu loss system begins to predict negative losses, explaining the unexpectedly low predicted profile losses.

Additionally, the transonic loss predictions did not capture the trends observed in the experimental data. A small dip in losses just below Mach 1.0 predicted by the loss system was not observed experimentally. Furthermore, measurements showed a tendency for losses to level off in a plateau at low supersonic Mach numbers which contrasts with the rapid monotonic rise in losses predicted by Kacker \& Okapuu. Unguided turning and suction surface curvature downstream of the throat appears to be in some way related to the extent of the loss plateau. The effect is possibly related to the strength and angle at which the impinging shock wave from the adjacent blade meets the suction surface. These questions are currently being investigated in a follow-up thesis.

The Reynolds number correction factor for the Kacker \& Okapuu predictions was also examined. Additionally, an alternative correction due to Aungier (2006), which accounts for roughness effects, was described. These two corrections were applied to the loss predictions for the rough cascade E2S50. It was found that the correction due to Aungier yields the best match to the experimental results when roughness effects are included.

\subsection{Recommendations for Future Work}

The findings of this thesis have shown that the widely used loss prediction system due to Kacker \& Okapuu is not satisfactory for the profile loss prediction of turbines with large negative inlet angles. Midspan losses were shown to be underpredicted for the three Siemens 
cascades measured. Since the Kacker \& Okapuu loss system is intended for use at design incidence, to improve the accuracy of the loss predictions at negative values of inlet angle, many cascades would need to be tested at their respective design conditions. Because manufacturing cascades is expensive, and testing cascades is time intensive, it is suggested that future studies turn to computational tools to expand the database. Computational fluid dynamics (CFD) offers the ability to obtain data for a large number of cases relatively quickly and cheaply compared with experiment. However, great care must be exercised to ensure accurate representation of the real cascade flow when modeling the turbine with CFD. Therefore, selective cascade testing should be used as validation for the computational research.

It is the opinion of the author that the plateau in losses observed at the supersonic Mach numbers is related to unguided turning of the blade and the surface curvature on the suction side of the airfoil downstream of the throat. Future work is required to confirm this relationship. Such work should focus on turbines designed for operation at high Mach numbers. Since the downstream measurements contain losses from all sources upstream, the effect of trailing edge blockage on supersonic losses could also be a factor. It was shown that the least accurate supersonic loss predictions were for the cascade with the thinnest trailing edge and lowest trailing edge blockage. Future work to determine the cause or causes of the loss plateau should in part be computational. CFD offers access to flow data at every point in the domain without measurement interference. In contrast, it can be very difficult, and time intensive to experimentally obtain detailed measurements, for example, of the blade surface boundary layer. Since the details of the shock patterns are expected to play a significant role in supersonic losses, future experimental work might consider optical techniques such as shadowgraph or Schlieren photography. 


\section{References}

Ainley, D. G. \& Mathieson, G. (1951). A method of performance estimation for axial-flow turbines. Aeronautical Research Council of Great Britain, R\&M 2974.

Amecke, J. \& Šafař́́k, P. (1995). Data reduction of wake flow measurements with injection of an other gas. Forschungsbericht DLR-FB 95-32, Cologne, Germany.

Aungier, R. H. (2006). Turbine Aerodynamics: Axial-Flow and Radial-Flow Turbine Design and Analysis. ASME Press.

Baines, N. C., King, P. I., Oldfield, M. L. G., Kiock, R., Hoheisel, H., Ramm, G., Lehthaus, F., Kost, F. H., \& Sieverding, C. H. (1986). A Comparison of Aerodynamic Measurements of the Transonic Flow Through a Plane Turbine Cascade in Four European Wind Tunnels. Technical Report Report No. OUEL 1624/86, Oxford University.

Baljé, O. E. \& Binsley, R. L. (1968). Axial turbine performance evaluation. ASME Paper $68-G T-13,$.

Benner, M. W., Sjolander, S. A., \& Moustapha, S. H. (1997). Influence of leading-edge geometry on profile losses in turbines at off-design incidence: Experimental results and an improved correlation. ASME Journal of Turbomachinery, Vol. 119, pp. 193-200.

Binder, A., Schroeder, T., \& Hourmouziadis, J. (1989). Turbulence measurements in a multistage low-pressure turbine. ASME Journal of Turbomachinery, Vol. 111, pp. 153-161.

Carter, A. D. S. \& Hughes, H. P. (1950). A theoretical investigation into the effects of profile shape on the performance of aerofoils in cascade. Aeronautical Research Council of Great Britain, R\&M 2384. 
Corriveau, D. (2005). Influence of loading distribution on the performance of high pressure turbine blades. Ph.D. thesis, Carleton University.

Corriveau, D. \& Sjolander, S. A. (2002). Impact of flow quality in transonic cacade wind tunnels: Measurements in an hp turbine cascade. Paper ICAS 2002-5.11.4.

Craig, H. R. M. \& Cox, H. J. A. (1971). Performance estimation of axial flow turbines. Proceedings of the Institute of Mechanical Engineers, Vol. 185 32/71, pp. 407-424.

Cumpsty, N. A. \& Horlock, J. H. (2006). Averaging nonuniform flow for a purpose. ASME Journal of Turbomachinery, Vol. 128, pp. 120-129.

Delery, J. \& Marvin, J. (1986). Shock-wave boundary layer interactions. AGARDograph, 280.

Denton, J. D. (1973). A survey and comparison of methods for predicting the profile loss of turbine blades. Institute of Mechanical Engineers Paper No. C76/73.

Denton, J. D. (1993). Loss mechanisms in turbomachines. ASME Journal of Turbomachinery, Vol. 115, pp. 621-656.

Denton, J. D. \& Xu, L. (1990). The trailing edge loss of transonic turbine blades. ASME Journal of Turbomachinery, Vol. 112, pp. 277-285.

Dunham, J. \& Came, P. M. (1970). Improvements to the ainley-mathieson method of turbine performance prediction. ASME Journal of Engineering for Gas Turbines and Power, Vol. 92 Ser A, pp. 252-256.

Graham, C. G. \& Kost, F. H. (1979). Shock boundary layer interaction on high turning transonic turbine cascades. ASME Paper 79-GT-3\%.

Hall, S. R. (2012). Contributions to High-Speed Linear Turbine Cascade Testing: Design of a Wind Tunnel Control System; and Investigation of the Effects of Compressibility on Total Pressure Losses. M.A.Sc. thesis, Carleton University. 
Hoheisel, H., Kiock, R., Lichtfuss, H. J., \& Fottner, L. (1987). Influence of free-stream turbulence and blade pressure gradient on boundary layer and loss behavior of turbine cascades. ASME Journal of Turbomachinery, Vol. 109, pp. 210-219.

Horlock, J. H. (1960). Losses and efficiencies in axial-flow turbines. International Journal of Mechanical Sciences, Vol. 2, pp. 48-75.

Hourmouziadis, J. (1989). Aerodynamic design of low pressure turbines. AGARD-LS-167.

Islam, A. M. T. (1999). An Experimental and Computational Study of the Aerodynamics of Turbine Blades With Damage. Ph.D. thesis, Carleton University.

Islam, A. M. T. \& Sjolander, S. A. (1999). Deviation in axial turbines at subsonic conditions. ASME Paper 99-GT-26.

Jeffries, M. S. (1994). The Commissioning and Enhancement of a Blow Down Wind Tunnel. M.A.Sc. thesis, Carleton University.

Jeffries, M. S. (2000). Initial Investigations of Transonic Turbine Aerodynamics using the Carleton University High-Speed Wind Tunnel. Ph.D. thesis, Carleton University.

Jeffries, M. S., Jouini, D., \& Sjolander, S. A. (1997). Determining sampling rates and times in a high speed wind tunnel. CASI 6th Symposium on Aerodynamics, Toronto.

Jeffries, M. S. \& Sjolander, S. A. (1995). The carleton university high-speed wind tunnel. CASI 7th Symposium on Propulsion, Montreal.

Jouini, D. B., Islam, A. M. T., \& Sjolander, S. A. (1998). Effect of surface roughness on profile losses in axial turbines at transonic mach numbers. Canadian Aeronautics and Space Journal, Vol. 44, pp. 263-272.

Jouini, D. B. M. (2000). Experimental Investigation of Two Transonic Linear Turbine Cascades at Off-Design Conditions. Ph.D. thesis, Carleton University. 
Jouini, D. B. M., Sjolander, S. A., \& Moustapha, S. H. (2002). Midspan flow-field measurements for two transonic linear turbine cascades at off-design conditions. ASME Journal of Turbomachinery, Vol. 124, pp. 176-186.

Kacker, S. C. \& Okapuu, U. (1982). A mean line prediction method for axial flow turbine efficiency. ASME Journal of Engineering for Gas Turbines and Power, Vol. 104, pp. 111119.

Kind, R. J., Serjak, P. J., \& Abbott, M. W. P. (1998). Measurements and prediction of the effects of surface roughness on profile losses and deviation in a turbine cascade. ASME Journal of Turbomachinery, Vol. 120, pp. 20-27.

Koch, C. C. \& Smith, L. H. (1976). Loss sources and magnitudes in axial-flow compressors. ASME Journal of Engineering for Gas Turbines and Power, Vol. 98, pp. 441-424.

Ladwig, M. \& Fottner, L. (1993). Experimental investigations of the influence of incoming wakes on the losses of a linear turbine cascade. ASME Paper 93-GT-394.

Mayle, R. E. (1991). The role of laminar-turbulent transition in gas turbine engines. ASME Journal of Turbomachinery, Vol. 113, pp. 509 - 537.

Mee, D. J., Baines, N. C., Oldfield, M. L. G., \& Dickens, T. E. (1992). An examination of the contributions to a loss on a transonic turbine in cascade. ASME Journal of Turbomachinery, Vol. 114, pp. 155-162.

Moustapha, S. H., Tremblay, B., \& Kacker, S. C. (1990). An improved incidence losses prediction method for turbine airfoils. ASME Journal of Turbomachinery, Vol. 112, pp. $267-276$.

Schlichting, H. (1962). Boundary Layer Theory. McGraw-Hill.

Sieverding, C. H. (1985). Thermodynamics and Fluid Mechanics of Turbomachinery Volume II, chapter Axial Turbine Performance Prediction Methods, (pp. 737-784). Martinus Nijhoff Publishers: Dordrecht. 
Sieverding, C. H., Stanislis, M., \& Snoek, J. (1979). The base pressure problem in transonic cascades. ASME Paper 79-GT-120.

Smith, S. F. (1965). A simple correlation of turbine efficiency. Journal of Royal Aeronautical Society, Vol. 60, pp. 467-470.

Starken, H. \& Lichtfuss, H. J. (1975). Aerodynamic measurements in cascades. AGARDograph, 205.

Stewart, W. L. (1961). A study of axial-flow turbine efficiency characteristics in terms of velocity diagram parameters. ASME Paper 61-WA-3\%.

Tarada, F. \& Suzuki, M. (1993). External heat transfer enhancement to trubine blading due to surface roughness. ASME Paper No. 93-GT-74.

Taremi, F., Sjolander, S. A., \& Abo El Ella, H. M. (2008). Extending the capabilities of a high-speed wind tunnel to secondary flow measurements in transonic linear turbine cascades. AIAA Paper No. 5272.

Taylor, R. P. (1990). Surface roughness measurements on gas turbine blades. ASME Journal of Turbomachinery, Vol. 112, pp. 175-180.

Traupel (1966). Thermische Turbomaschinen. Berlin: Springer-Verlag. (As referenced by Denton (1973)).

White, F. M. (2006). Fluid Mechanics. McGraw-Hill, 6th edition.

Yuan, L. Q. (2004). Measurements and Computations of Roughness Effects on Performance of a HP Turbine Cascade in Compressible Flow. M.A.Sc. thesis, Carleton University.

Zhang, Q. \& Ligrani, P. M. (2004). Mach number/surface roughness effects on symmetric transonic turbine airfoil aerodynamic losses. AIAA Journal of Propulsion and Power, Vol. 20, pp. $1117-1125$. 
Zhang, Q. \& Ligrani, P. M. (2006). Aerodynamic losses of a cambered turbine vane: Influences of surface roughness and freestream turbulence intensity. ASME Journal of Turbomachinery, Vol. 128, pp. 536-546.

Zhu, J. \& Sjolander, S. A. (2005). Improved profile loss and deviation correlations for axialturbine blade rows. ASME Paper No. GT2005-6907\%. 


\section{Appendix A}

\section{Siemens Cascade Geometry}

Table A.1: Siemens Cascade Coordinates

\begin{tabular}{|c|c|c|c|c|c|c|c|}
\hline \multicolumn{2}{|c|}{ E1S50 } & \multicolumn{2}{|c|}{ E1S90 } & \multicolumn{2}{|c|}{ E2S50 } & \multicolumn{2}{|c|}{ E2S90 } \\
\hline$x / c_{x}$ & $y / c_{x}$ & $x / c_{x}$ & $y / c_{x}$ & $x / c_{x}$ & $y / c_{x}$ & $x / c_{x}$ & $y / c_{x}$ \\
\hline 1.0016 & 0.0071 & 1.0069 & 0.0099 & 1.0020 & 0.0170 & 1.0052 & 0.0232 \\
\hline 0.9986 & 0.0122 & 1.0039 & 0.0177 & 0.9987 & 0.0212 & 1.0020 & 0.0289 \\
\hline 0.9950 & 0.0182 & 1.0004 & 0.0269 & 0.9948 & 0.0261 & 0.9982 & 0.0355 \\
\hline 0.9924 & 0.0227 & 0.9978 & 0.0337 & 0.9919 & 0.0297 & 0.9954 & 0.0405 \\
\hline 0.9880 & 0.0301 & 0.9934 & 0.0450 & 0.9871 & 0.0358 & 0.9907 & 0.0486 \\
\hline 0.9830 & 0.0383 & 0.9884 & 0.0576 & 0.9818 & 0.0425 & 0.9855 & 0.0578 \\
\hline 0.9795 & 0.0443 & 0.9849 & 0.0668 & 0.9779 & 0.0474 & 0.9816 & 0.0644 \\
\hline 0.9736 & 0.0540 & 0.9790 & 0.0815 & 0.9716 & 0.0553 & 0.9755 & 0.0750 \\
\hline 0.9673 & 0.0644 & 0.9727 & 0.0976 & 0.9647 & 0.0638 & 0.9687 & 0.0866 \\
\hline 0.9628 & 0.0719 & 0.9682 & 0.1090 & 0.9598 & 0.0699 & 0.9639 & 0.0949 \\
\hline 0.9555 & 0.0838 & 0.9611 & 0.1272 & 0.9520 & 0.0796 & 0.9562 & 0.1080 \\
\hline 0.9478 & 0.0965 & 0.9535 & 0.1468 & 0.9436 & 0.0899 & 0.9479 & 0.1220 \\
\hline 0.9423 & 0.1054 & 0.9481 & 0.1605 & 0.9376 & 0.0972 & 0.9421 & 0.1319 \\
\hline 0.9336 & 0.1195 & 0.9397 & 0.1823 & 0.9283 & 0.1087 & 0.9328 & 0.1474 \\
\hline 0.9244 & 0.1344 & 0.9308 & 0.2053 & 0.9183 & 0.1208 & 0.9229 & 0.1639 \\
\hline 0.9180 & 0.1448 & 0.9246 & 0.2214 & 0.9113 & 0.1292 & 0.9159 & 0.1753 \\
\hline 0.9079 & 0.1612 & 0.9148 & 0.2466 & 0.9003 & 0.1424 & 0.9050 & 0.1932 \\
\hline 0.8973 & 0.1784 & 0.9044 & 0.2730 & 0.8888 & 0.1562 & 0.8934 & 0.2120 \\
\hline 0.8900 & 0.1903 & 0.8972 & 0.2913 & 0.8807 & 0.1658 & 0.8854 & 0.2251 \\
\hline
\end{tabular}


Table A.1: Siemens Cascade Coordinates

\begin{tabular}{|c|c|c|c|c|c|c|c|}
\hline \multicolumn{2}{|c|}{ E1S50 } & \multicolumn{2}{|c|}{ E1S90 } & \multicolumn{2}{|c|}{ E2S50 } & \multicolumn{2}{|c|}{ E2S90 } \\
\hline$x / c_{x}$ & $y / c_{x}$ & $x / c_{x}$ & $y / c_{x}$ & $x / c_{x}$ & $y / c_{x}$ & $x / c_{x}$ & $y / c_{x}$ \\
\hline 0.8785 & 0.2089 & 0.8859 & 0.3200 & 0.8681 & 0.1806 & 0.8727 & 0.2453 \\
\hline 0.8666 & 0.2283 & 0.8742 & 0.3499 & 0.8549 & 0.1961 & 0.8594 & 0.2664 \\
\hline 0.8584 & 0.2418 & 0.8661 & 0.3706 & 0.8457 & 0.2068 & 0.8502 & 0.2810 \\
\hline 0.8456 & 0.2627 & 0.8537 & 0.4027 & 0.8314 & 0.2232 & 0.8357 & 0.3035 \\
\hline 0.8324 & 0.2845 & 0.8407 & 0.4361 & 0.8164 & 0.2403 & 0.8206 & 0.3269 \\
\hline 0.8234 & 0.2995 & 0.8318 & 0.4592 & 0.8061 & 0.2519 & 0.8102 & 0.3430 \\
\hline 0.8094 & 0.3227 & 0.8181 & 0.4948 & 0.7900 & 0.2698 & 0.7939 & 0.3678 \\
\hline 0.7999 & 0.3387 & 0.8087 & 0.5193 & 0.7788 & 0.2821 & 0.7826 & 0.3847 \\
\hline 0.7853 & 0.3635 & 0.7942 & 0.5571 & 0.7615 & 0.3008 & 0.7652 & 0.4109 \\
\hline 0.7702 & 0.3892 & 0.7792 & 0.5963 & 0.7434 & 0.3200 & 0.7470 & 0.4379 \\
\hline 0.7599 & 0.4068 & 0.7689 & 0.6231 & 0.7308 & 0.3329 & 0.7345 & 0.4563 \\
\hline 0.7440 & 0.4339 & 0.7531 & 0.6644 & 0.7113 & 0.3527 & 0.7151 & 0.4846 \\
\hline 0.7276 & 0.4618 & 0.7368 & 0.7070 & 0.6909 & 0.3727 & 0.6950 & 0.5137 \\
\hline 0.7163 & 0.4809 & 0.7256 & 0.7361 & 0.6768 & 0.3861 & 0.6813 & 0.5335 \\
\hline 0.6989 & 0.5102 & 0.7084 & 0.7809 & 0.6548 & 0.4063 & 0.6600 & 0.5640 \\
\hline 0.6806 & 0.5401 & 0.6906 & 0.8270 & 0.6317 & 0.4265 & 0.6381 & 0.5952 \\
\hline 0.6679 & 0.5603 & 0.6784 & 0.8584 & 0.6157 & 0.4399 & 0.6230 & 0.6165 \\
\hline 0.6478 & 0.5910 & 0.6596 & 0.9065 & 0.5907 & 0.4598 & 0.6000 & 0.6491 \\
\hline 0.6263 & 0.6220 & 0.6399 & 0.9558 & 0.5645 & 0.4793 & 0.5762 & 0.6825 \\
\hline 0.6113 & 0.6425 & 0.6264 & 0.9889 & 0.5465 & 0.4918 & 0.5601 & 0.7049 \\
\hline 0.5883 & 0.6719 & 0.6063 & 1.0375 & 0.5196 & 0.5093 & 0.5365 & 0.7379 \\
\hline 0.5646 & 0.6996 & 0.5863 & 1.0847 & 0.4928 & 0.5251 & 0.5133 & 0.7698 \\
\hline 0.5486 & 0.7170 & 0.5731 & 1.1153 & 0.4750 & 0.5347 & 0.4982 & 0.7905 \\
\hline 0.5240 & 0.7415 & 0.5536 & 1.1601 & 0.4487 & 0.5477 & 0.4759 & 0.8208 \\
\hline 0.4990 & 0.7641 & 0.5343 & 1.2035 & 0.4226 & 0.5590 & 0.4541 & 0.8500 \\
\hline 0.4821 & 0.7780 & 0.5214 & 1.2316 & 0.4054 & 0.5656 & 0.4398 & 0.8688 \\
\hline 0.4564 & \begin{tabular}{|l|}
0.7972 \\
\end{tabular} & 0.5016 & 1.2722 & 0.3801 & 0.5742 & 0.4187 & 0.8962 \\
\hline 0.4303 & 0.8142 & 0.4814 & 1.3110 & 0.3552 & 0.5813 & 0.3980 & 0.9224 \\
\hline 0.4129 & 0.8243 & 0.4677 & 1.3359 & 0.3390 & 0.5852 & 0.3843 & 0.9393 \\
\hline 0.3867 & 0.8376 & 0.4468 & 1.3717 & 0.3152 & 0.5899 & 0.3641 & 0.9635 \\
\hline
\end{tabular}


Table A.1: Siemens Cascade Coordinates

\begin{tabular}{|c|c|c|c|c|c|c|c|}
\hline \multicolumn{2}{|c|}{ E1S50 } & \multicolumn{2}{|c|}{ E1S90 } & \multicolumn{2}{|c|}{ E2S50 } & \multicolumn{2}{|c|}{ E2S90 } \\
\hline$x / c_{x}$ & $y / c_{x}$ & $x / c_{x}$ & $y / c_{x}$ & $x / c_{x}$ & $y / c_{x}$ & $x / c_{x}$ & $y / c_{x}$ \\
\hline 0.3605 & 0.8487 & 0.4254 & 1.4056 & 0.2921 & 0.5932 & 0.3441 & 0.9865 \\
\hline 0.3432 & 0.8550 & 0.4110 & 1.4271 & 0.2772 & 0.5948 & 0.3309 & 1.0011 \\
\hline 0.3175 & 0.8626 & 0.3890 & 1.4577 & 0.2554 & 0.5962 & 0.3114 & 1.0220 \\
\hline 0.3007 & 0.8666 & 0.3742 & 1.4771 & 0.2413 & 0.5966 & 0.2985 & 1.0352 \\
\hline 0.2759 & 0.8710 & 0.3518 & 1.5044 & 0.2210 & 0.5964 & 0.2793 & 1.0538 \\
\hline 0.2519 & 0.8735 & 0.3292 & 1.5299 & 0.2015 & 0.5954 & 0.2604 & 1.0711 \\
\hline 0.2364 & 0.8744 & 0.3142 & 1.5458 & 0.1890 & 0.5943 & 0.2480 & 1.0818 \\
\hline 0.2139 & 0.8744 & 0.2917 & 1.5681 & 0.1710 & 0.5921 & 0.2295 & 1.0966 \\
\hline 0.1925 & 0.8732 & 0.2693 & 1.5885 & 0.1540 & 0.5894 & 0.2114 & 1.1101 \\
\hline 0.1787 & 0.8719 & 0.2546 & 1.6011 & 0.1431 & 0.5873 & 0.1995 & 1.1183 \\
\hline 0.1591 & 0.8690 & 0.2327 & 1.6184 & 0.1275 & 0.5838 & 0.1819 & 1.1295 \\
\hline 0.1406 & 0.8654 & 0.2111 & 1.6340 & 0.1129 & 0.5800 & 0.1647 & 1.1392 \\
\hline 0.1289 & 0.8627 & 0.1970 & 1.6433 & 0.1036 & 0.5773 & 0.1535 & 1.1449 \\
\hline 0.1122 & 0.8583 & 0.1764 & 1.6560 & 0.0905 & 0.5731 & 0.1370 & 1.1522 \\
\hline 0.0967 & 0.8536 & 0.1563 & 1.6669 & 0.0782 & 0.5687 & 0.1212 & 1.1581 \\
\hline 0.0870 & 0.8504 & 0.1434 & 1.6734 & 0.0705 & 0.5657 & 0.1109 & 1.1612 \\
\hline 0.0733 & 0.8455 & 0.1247 & 1.6818 & 0.0597 & 0.5612 & 0.0961 & 1.1648 \\
\hline 0.0606 & 0.8405 & 0.1069 & 1.6889 & 0.0498 & 0.5566 & 0.0821 & 1.1671 \\
\hline 0.0528 & 0.8372 & 0.0957 & 1.6929 & 0.0436 & 0.5536 & 0.0732 & 1.1681 \\
\hline 0.0419 & 0.8324 & 0.0797 & 1.6981 & 0.0351 & 0.5490 & 0.0606 & 1.1686 \\
\hline 0.0319 & 0.8279 & 0.0648 & 1.7021 & 0.0275 & 0.5446 & 0.0490 & 1.1681 \\
\hline 0.0258 & 0.8250 & 0.0556 & 1.7042 & 0.0229 & 0.5417 & 0.0419 & 1.1674 \\
\hline 0.0177 & 0.8205 & 0.0427 & 1.7064 & 0.0167 & 0.5374 & 0.0324 & 1.1657 \\
\hline 0.0108 & 0.8160 & 0.0311 & 1.7075 & 0.0113 & 0.5333 & 0.0240 & 1.1635 \\
\hline 0.0070 & 0.8127 & 0.0241 & 1.7074 & 0.0083 & 0.5306 & 0.0191 & 1.1619 \\
\hline 0.0028 & 0.8076 & 0.0149 & 1.7056 & 0.0044 & 0.5268 & 0.0126 & 1.1595 \\
\hline 0.0010 & 0.8042 & 0.0099 & 1.7035 & 0.0023 & 0.5244 & 0.0089 & 1.1578 \\
\hline 0.0008 & 0.8038 & 0.0093 & 1.7031 & 0.0018 & 0.5238 & 0.0081 & 1.1574 \\
\hline 0.0006 & 0.8033 & 0.0086 & 1.7027 & 0.0014 & 0.5231 & 0.0073 & 1.1569 \\
\hline 0.0005 & 0.8029 & 0.0080 & 1.7023 & 0.0010 & 0.5224 & 0.0064 & 1.1563 \\
\hline
\end{tabular}


Table A.1: Siemens Cascade Coordinates

\begin{tabular}{|c|c|c|c|c|c|c|c|}
\hline \multicolumn{2}{|c|}{ E1S50 } & \multicolumn{2}{|c|}{ E1S90 } & \multicolumn{2}{|c|}{ E2S50 } & \multicolumn{2}{|c|}{ E2S90 } \\
\hline$x / c_{x}$ & $y / c_{x}$ & $x / c_{x}$ & $y / c_{x}$ & $x / c_{x}$ & $y / c_{x}$ & $x / c_{x}$ & $y / c_{x}$ \\
\hline 0.0004 & 0.8024 & 0.0073 & 1.7018 & 0.0007 & 0.5217 & 0.0056 & 1.1556 \\
\hline 0.0003 & 0.8020 & 0.0067 & 1.7013 & 0.0004 & 0.5209 & 0.0048 & 1.1549 \\
\hline 0.0002 & 0.8015 & 0.0061 & 1.7008 & 0.0002 & 0.5200 & 0.0040 & 1.1540 \\
\hline 0.0001 & 0.8011 & 0.0056 & 1.7002 & 0.0001 & 0.5192 & 0.0033 & 1.1531 \\
\hline 0.0000 & 0.8006 & 0.0050 & 1.6997 & 0.0000 & 0.5183 & 0.0026 & 1.1522 \\
\hline 0.0000 & 0.8001 & 0.0045 & 1.6991 & 0.0000 & 0.5175 & 0.0019 & 1.1511 \\
\hline 0.0000 & 0.7996 & 0.0040 & 1.6984 & 0.0001 & 0.5166 & 0.0014 & 1.1500 \\
\hline 0.0000 & 0.7991 & 0.0035 & 1.6978 & 0.0002 & 0.5158 & 0.0009 & 1.1488 \\
\hline 0.0000 & 0.7987 & 0.0030 & 1.6971 & 0.0004 & 0.5150 & 0.0005 & 1.1476 \\
\hline 0.0001 & 0.7982 & 0.0026 & 1.6964 & 0.0006 & 0.5142 & 0.0002 & 1.1463 \\
\hline 0.0002 & 0.7977 & 0.0022 & 1.6957 & 0.0010 & 0.5134 & 0.0000 & 1.1450 \\
\hline 0.0002 & 0.7972 & 0.0019 & 1.6950 & 0.0014 & 0.5127 & 0.0000 & 1.1437 \\
\hline 0.0004 & 0.7968 & 0.0015 & 1.6943 & 0.0018 & 0.5119 & 0.0001 & 1.1424 \\
\hline 0.0005 & 0.7963 & 0.0012 & 1.6935 & 0.0023 & 0.5112 & 0.0002 & 1.1412 \\
\hline 0.0006 & 0.7959 & 0.0010 & 1.6928 & 0.0029 & 0.5106 & 0.0004 & 1.1400 \\
\hline 0.0008 & 0.7954 & 0.0007 & 1.6920 & 0.0035 & 0.5100 & 0.0007 & 1.1389 \\
\hline 0.0010 & 0.7950 & 0.0005 & 1.6913 & 0.0041 & 0.5095 & 0.0011 & 1.1378 \\
\hline 0.0012 & 0.7945 & 0.0003 & 1.6905 & 0.0048 & 0.5090 & 0.0015 & 1.1367 \\
\hline 0.0014 & 0.7941 & 0.0002 & 1.6897 & 0.0055 & 0.5086 & 0.0019 & 1.1357 \\
\hline 0.0016 & 0.7937 & 0.0001 & 1.6890 & 0.0063 & 0.5082 & 0.0024 & 1.1348 \\
\hline 0.0019 & 0.7933 & 0.0000 & 1.6882 & 0.0070 & 0.5079 & 0.0030 & 1.1339 \\
\hline 0.0021 & 0.7930 & 0.0000 & 1.6875 & 0.0076 & 0.5077 & 0.0034 & 1.1333 \\
\hline 0.0058 & 0.7892 & 0.0010 & 1.6796 & 0.0119 & 0.5067 & 0.0073 & 1.1286 \\
\hline 0.0112 & 0.7861 & 0.0044 & 1.6709 & 0.0168 & 0.5056 & 0.0122 & 1.1235 \\
\hline 0.0155 & 0.7847 & 0.0080 & 1.6649 & 0.0206 & 0.5048 & 0.0160 & 1.1199 \\
\hline 0.0231 & 0.7832 & 0.0147 & 1.6554 & 0.0268 & 0.5037 & 0.0225 & 1.1142 \\
\hline 0.0316 & 0.7821 & 0.0226 & 1.6451 & 0.0337 & 0.5026 & 0.0302 & 1.1082 \\
\hline 0.0378 & 0.7814 & 0.0285 & 1.6378 & 0.0388 & 0.5019 & 0.0358 & 1.1040 \\
\hline 0.0478 & 0.7802 & 0.0380 & 1.6260 & 0.0470 & 0.5009 & 0.0451 & 1.0974 \\
\hline 0.0586 & 0.7787 & 0.0484 & 1.6132 & 0.0559 & 0.4999 & 0.0553 & 1.0905 \\
\hline
\end{tabular}


Table A.1: Siemens Cascade Coordinates

\begin{tabular}{|c|c|c|c|c|c|c|c|}
\hline \multicolumn{2}{|c|}{ E1S50 } & \multicolumn{2}{|c|}{ E1S90 } & \multicolumn{2}{|c|}{ E2S50 } & \multicolumn{2}{|c|}{ E2S90 } \\
\hline$x / c_{x}$ & $y / c_{x}$ & $x / c_{x}$ & $y / c_{x}$ & $x / c_{x}$ & $y / c_{x}$ & $x / c_{x}$ & $y / c_{x}$ \\
\hline 0.0662 & 0.7774 & 0.0557 & 1.6040 & 0.0623 & 0.4993 & 0.0626 & 1.0855 \\
\hline 0.0784 & 0.7749 & 0.0673 & 1.5893 & 0.0724 & 0.4983 & 0.0742 & 1.0776 \\
\hline 0.0913 & 0.7718 & 0.0794 & 1.5733 & 0.0832 & 0.4973 & 0.0866 & 1.0691 \\
\hline 0.1003 & \begin{tabular}{|l}
0.7694 \\
\end{tabular} & 0.0877 & 1.5619 & 0.0909 & 0.4967 & 0.0953 & 1.0631 \\
\hline 0.1145 & \begin{tabular}{|l|}
0.7651 \\
\end{tabular} & 0.1008 & 1.5439 & 0.1029 & 0.4956 & 0.1089 & 1.0534 \\
\hline 0.1293 & 0.7601 & 0.1144 & 1.5246 & 0.1157 & 0.4945 & 0.1233 & 1.0431 \\
\hline 0.1396 & 0.7564 & 0.1238 & 1.5110 & 0.1246 & 0.4937 & 0.1333 & 1.0358 \\
\hline 0.1555 & 0.7502 & 0.1384 & 1.4897 & 0.1385 & 0.4923 & 0.1489 & 1.0242 \\
\hline 0.1721 & 0.7432 & 0.1536 & 1.4672 & 0.1532 & 0.4908 & 0.1652 & 1.0118 \\
\hline 0.1835 & 0.7381 & 0.1641 & 1.4515 & 0.1635 & 0.4897 & 0.1764 & 1.0031 \\
\hline 0.2012 & 0.7299 & 0.1803 & 1.4270 & 0.1794 & 0.4878 & 0.1939 & 0.9894 \\
\hline 0.2194 & 0.7209 & 0.1971 & 1.4012 & 0.1962 & 0.4856 & 0.2120 & 0.9748 \\
\hline 0.2319 & 0.7144 & 0.2086 & 1.3834 & 0.2078 & 0.4839 & 0.2244 & 0.9646 \\
\hline 0.2511 & 0.7041 & 0.2264 & 1.3557 & 0.2258 & 0.4811 & 0.2435 & 0.9486 \\
\hline 0.2709 & \begin{tabular}{|l}
0.6929 \\
\end{tabular} & 0.2449 & 1.3267 & 0.2446 & 0.4779 & 0.2632 & 0.9316 \\
\hline 0.2844 & 0.6850 & 0.2575 & 1.3068 & 0.2575 & 0.4754 & 0.2767 & 0.9198 \\
\hline 0.3051 & 0.6725 & 0.2768 & 1.2758 & 0.2775 & 0.4713 & 0.2974 & 0.9012 \\
\hline 0.3192 & 0.6637 & 0.2901 & 1.2545 & 0.2912 & 0.4682 & 0.3115 & 0.8883 \\
\hline 0.3408 & 0.6498 & 0.3105 & 1.2215 & 0.3124 & 0.4631 & 0.3330 & 0.8680 \\
\hline 0.3629 & 0.6350 & 0.3314 & 1.1874 & 0.3342 & 0.4573 & 0.3549 & 0.8467 \\
\hline 0.3779 & 0.6247 & 0.3457 & 1.1639 & 0.3491 & 0.4530 & 0.3698 & 0.8318 \\
\hline 0.4008 & 0.6086 & 0.3677 & 1.1278 & 0.3721 & 0.4459 & 0.3925 & 0.8087 \\
\hline 0.4241 & 0.5916 & 0.3902 & 1.0904 & 0.3956 & 0.4380 & 0.4156 & 0.7844 \\
\hline 0.4400 & 0.5797 & 0.4056 & 1.0648 & 0.4116 & 0.4322 & 0.4311 & 0.7676 \\
\hline 0.4641 & 0.5613 & 0.4292 & 1.0255 & 0.4361 & 0.4228 & 0.4547 & 0.7414 \\
\hline 0.4886 & 0.5419 & 0.4533 & 0.9849 & 0.4612 & 0.4123 & 0.4785 & 0.7140 \\
\hline 0.5052 & 0.5285 & 0.4697 & 0.9572 & 0.4782 & 0.4048 & 0.4945 & 0.6951 \\
\hline 0.5305 & 0.5077 & 0.4947 & 0.9145 & 0.5042 & 0.3925 & 0.5187 & 0.6657 \\
\hline 0.5561 & 0.4859 & 0.5203 & 0.8707 & 0.5306 & 0.3792 & 0.5431 & 0.6352 \\
\hline 0.5732 & 0.4710 & 0.5375 & 0.8411 & 0.5483 & 0.3697 & 0.5593 & 0.6143 \\
\hline
\end{tabular}


Table A.1: Siemens Cascade Coordinates

\begin{tabular}{|c|c|c|c|c|c|c|c|}
\hline \multicolumn{2}{|c|}{ E1S50 } & \multicolumn{2}{|c|}{ E1S90 } & \multicolumn{2}{|c|}{ E2S50 } & \multicolumn{2}{|c|}{ E2S90 } \\
\hline$x / c_{x}$ & $y / c_{x}$ & $x / c_{x}$ & $y / c_{x}$ & $x / c_{x}$ & $y / c_{x}$ & $x / c_{x}$ & $y / c_{x}$ \\
\hline 0.5982 & 0.4489 & 0.5627 & 0.7975 & 0.5740 & 0.3551 & 0.5827 & 0.5834 \\
\hline 0.6222 & 0.4271 & 0.5871 & 0.7550 & 0.5988 & 0.3402 & 0.6052 & 0.5530 \\
\hline 0.6377 & 0.4127 & 0.6030 & 0.7273 & 0.6148 & 0.3301 & 0.6197 & 0.5331 \\
\hline 0.6602 & 0.3915 & 0.6261 & 0.6867 & 0.6379 & 0.3149 & 0.6407 & 0.5037 \\
\hline 0.6819 & 0.3706 & 0.6484 & 0.6471 & 0.6602 & 0.2995 & 0.6608 & 0.4749 \\
\hline 0.6959 & 0.3569 & 0.6629 & 0.6214 & 0.6745 & 0.2892 & 0.6739 & 0.4562 \\
\hline 0.7161 & 0.3366 & 0.6840 & 0.5837 & 0.6952 & 0.2739 & 0.6928 & 0.4287 \\
\hline 0.7355 & 0.3168 & 0.7044 & 0.5471 & 0.7151 & 0.2586 & 0.7111 & 0.4019 \\
\hline 0.7480 & 0.3039 & 0.7176 & 0.5234 & 0.7279 & 0.2484 & 0.7229 & 0.3845 \\
\hline 0.7660 & 0.2848 & 0.7367 & 0.4886 & 0.7463 & 0.2334 & 0.7401 & 0.3591 \\
\hline 0.7833 & 0.2662 & 0.7552 & 0.4551 & 0.7639 & 0.2185 & 0.7567 & 0.3345 \\
\hline 0.7944 & 0.2541 & 0.7671 & 0.4333 & 0.7753 & 0.2088 & 0.7674 & 0.3185 \\
\hline 0.8103 & 0.2363 & 0.7843 & 0.4016 & 0.7916 & 0.1944 & 0.7830 & 0.2953 \\
\hline 0.8206 & 0.2247 & 0.7954 & 0.3811 & 0.8021 & 0.1850 & 0.7931 & 0.2803 \\
\hline 0.8353 & 0.2078 & 0.8115 & 0.3514 & 0.8172 & 0.1712 & 0.8077 & 0.2585 \\
\hline 0.8493 & 0.1915 & 0.8269 & 0.3227 & 0.8316 & 0.1578 & 0.8217 & 0.2376 \\
\hline 0.8583 & 0.1809 & 0.8368 & 0.3043 & 0.8408 & 0.1491 & 0.8308 & 0.2241 \\
\hline 0.8711 & 0.1654 & 0.8511 & 0.2775 & 0.8540 & 0.1365 & 0.8439 & 0.2046 \\
\hline 0.8833 & 0.1506 & 0.8647 & 0.2520 & 0.8666 & 0.1243 & 0.8564 & 0.1860 \\
\hline 0.8910 & 0.1410 & 0.8734 & 0.2356 & 0.8747 & 0.1164 & 0.8645 & 0.1741 \\
\hline 0.9021 & 0.1272 & 0.8859 & 0.2119 & 0.8862 & 0.1050 & 0.8760 & 0.1569 \\
\hline 0.9125 & 0.1140 & 0.8977 & 0.1894 & 0.8971 & 0.0941 & 0.8871 & 0.1405 \\
\hline 0.9190 & 0.1055 & 0.9052 & 0.1751 & 0.9040 & 0.0871 & 0.8941 & 0.1301 \\
\hline 0.9284 & 0.0933 & 0.9160 & 0.1546 & 0.9139 & 0.0772 & 0.9042 & 0.1152 \\
\hline 0.9371 & 0.0818 & 0.9261 & 0.1352 & 0.9232 & 0.0677 & 0.9137 & 0.1012 \\
\hline 0.9426 & 0.0744 & 0.9324 & 0.1229 & 0.9291 & 0.0617 & 0.9197 & 0.0924 \\
\hline 0.9503 & 0.0640 & 0.9415 & 0.1055 & 0.9374 & 0.0532 & 0.9283 & 0.0798 \\
\hline 0.9574 & 0.0542 & 0.9499 & 0.0893 & 0.9452 & 0.0452 & 0.9363 & 0.0680 \\
\hline 0.9618 & 0.0480 & 0.9551 & 0.0791 & 0.9500 & 0.0402 & 0.9413 & 0.0607 \\
\hline 0.9680 & 0.0393 & 0.9624 & 0.0648 & 0.9568 & 0.0332 & 0.9483 & 0.0504 \\
\hline
\end{tabular}


Table A.1: Siemens Cascade Coordinates

\begin{tabular}{|c|c|c|c|c|c|c|c|}
\hline \multicolumn{2}{|c|}{ E1S50 } & \multicolumn{2}{|c|}{ E1S90 } & \multicolumn{2}{|c|}{ E2S50 } & \multicolumn{2}{|c|}{ E2S90 } \\
\hline$x / c_{x}$ & $y / c_{x}$ & $x / c_{x}$ & $y / c_{x}$ & $x / c_{x}$ & $y / c_{x}$ & $x / c_{x}$ & $y / c_{x}$ \\
\hline 0.9736 & 0.0314 & 0.9692 & 0.0517 & 0.9630 & 0.0268 & 0.9548 & 0.0410 \\
\hline 0.9770 & 0.0264 & 0.9733 & 0.0436 & 0.9669 & 0.0228 & 0.9587 & 0.0352 \\
\hline 0.9817 & 0.0196 & 0.9790 & 0.0325 & 0.9722 & 0.0173 & 0.9642 & 0.0272 \\
\hline 0.9859 & 0.0135 & 0.9840 & 0.0226 & 0.9769 & 0.0124 & 0.9691 & 0.0200 \\
\hline 0.9884 & 0.0098 & 0.9871 & 0.0166 & 0.9797 & 0.0095 & 0.9721 & 0.0158 \\
\hline 0.9917 & 0.0049 & 0.9911 & 0.0086 & 0.9835 & 0.0056 & 0.9760 & 0.0100 \\
\hline 0.9936 & 0.0021 & 0.9935 & 0.0040 & 0.9857 & 0.0033 & 0.9783 & 0.0067 \\
\hline 0.9940 & 0.0016 & 0.9939 & 0.0032 & 0.9867 & 0.0024 & 0.9796 & 0.0051 \\
\hline 0.9944 & 0.0012 & 0.9945 & 0.0025 & 0.9877 & 0.0016 & 0.9809 & 0.0038 \\
\hline 0.9948 & 0.0008 & 0.9951 & 0.0018 & 0.9889 & 0.0010 & 0.9824 & 0.0027 \\
\hline 0.9954 & 0.0005 & 0.9959 & 0.0012 & 0.9903 & 0.0005 & 0.9843 & 0.0016 \\
\hline 0.9960 & 0.0002 & 0.9967 & 0.0008 & 0.9916 & 0.0002 & 0.9862 & 0.0008 \\
\hline 0.9965 & 0.0001 & 0.9976 & 0.0004 & 0.9930 & 0.0000 & 0.9881 & 0.0003 \\
\hline 0.9972 & 0.0000 & 0.9986 & 0.0001 & 0.9945 & 0.0000 & 0.9903 & 0.0000 \\
\hline 0.9978 & 0.0000 & 0.9995 & 0.0000 & 0.9958 & 0.0003 & 0.9924 & 0.0000 \\
\hline 0.9984 & 0.0001 & 1.0004 & 0.0000 & 0.9971 & 0.0006 & 0.9943 & 0.0003 \\
\hline 0.9990 & 0.0002 & 1.0013 & 0.0001 & 0.9983 & 0.0011 & 0.9962 & 0.0007 \\
\hline 0.9996 & 0.0004 & 1.0022 & 0.0003 & 0.9996 & 0.0018 & 0.9982 & 0.0014 \\
\hline 1.0001 & 0.0007 & 1.0030 & 0.0006 & 1.0005 & 0.0026 & 0.9998 & 0.0023 \\
\hline 1.0005 & 0.0010 & 1.0038 & 0.0010 & 1.0014 & 0.0034 & 1.0012 & 0.0033 \\
\hline 1.0009 & 0.0015 & 1.0045 & 0.0016 & 1.0023 & 0.0045 & 1.0028 & 0.0048 \\
\hline 1.0013 & 0.0019 & 1.0052 & 0.0022 & 1.0030 & 0.0056 & 1.0040 & 0.0063 \\
\hline 1.0016 & 0.0024 & 1.0058 & 0.0029 & 1.0035 & 0.0068 & 1.0051 & 0.0080 \\
\hline 1.0019 & 0.0030 & 1.0063 & 0.0036 & 1.0040 & 0.0081 & 1.0060 & 0.0097 \\
\hline 1.0021 & 0.0036 & 1.0068 & 0.0046 & 1.0042 & 0.0096 & 1.0067 & 0.0119 \\
\hline 1.0022 & 0.0042 & 1.0071 & 0.0054 & 1.0042 & 0.0109 & 1.0071 & 0.0138 \\
\hline 1.0022 & 0.0048 & 1.0073 & 0.0063 & 1.0041 & 0.0122 & 1.0072 & 0.0158 \\
\hline 1.0022 & 0.0055 & 1.0073 & 0.0073 & 1.0037 & 0.0137 & 1.0070 & 0.0180 \\
\hline 1.0020 & 0.0061 & 1.0073 & 0.0082 & 1.0033 & 0.0149 & 1.0066 & 0.0198 \\
\hline 1.0018 & 0.0066 & 1.0071 & 0.0091 & 1.0027 & 0.0160 & 1.0060 & 0.0216 \\
\hline
\end{tabular}


Table A.1: Siemens Cascade Coordinates

\begin{tabular}{|c|c|c|c|c|c|c|c|c|c|}
\hline \multicolumn{2}{|c|}{ E1S50 } & & \multicolumn{2}{c|}{ E1S90 } & & \multicolumn{2}{c|}{ E2S50 } & \multicolumn{2}{c|}{ E2S90 } \\
\hline $\boldsymbol{x} / \boldsymbol{c}_{\boldsymbol{x}}$ & $\boldsymbol{y} / \boldsymbol{c}_{\boldsymbol{x}}$ & & $\boldsymbol{x} / \boldsymbol{c}_{\boldsymbol{x}}$ & $\boldsymbol{y} / \boldsymbol{c}_{\boldsymbol{x}}$ & & $\boldsymbol{x} / \boldsymbol{c}_{\boldsymbol{x}}$ & $\boldsymbol{y} / \boldsymbol{c}_{\boldsymbol{x}}$ & $\boldsymbol{x} / \boldsymbol{c}_{\boldsymbol{x}}$ & $\boldsymbol{y} / \boldsymbol{c}_{\boldsymbol{x}}$ \\
\hline \hline 1.0016 & 0.0071 & & 1.0069 & 0.0099 & & 1.0020 & 0.0170 & 1.0052 & 0.0232 \\
\hline
\end{tabular}




\section{Appendix B}

\section{Amecke \& Šafařík Method for Mixing-Out Non-Uniform Flows}

To solve for the mixed-out properties, the equations of conservation of mass, momentum and energy are solved for a control volume that extends from the measuring plane to a hypothetical downstream plane (the mixed-out plane in Figure 4.6), at which the flow properties are homogenous. A detailed derivation of the conservation equations for the flow field of a turbomachine can be found in Amecke \& Šafař́k (1995). The Amecke \& Šafař́k derivations assume the flow to behave as a perfect gas. Therefore, the following expressions are assumed to hold true:

$$
\frac{P}{\rho}=R T
$$

and

$$
\gamma=\frac{c_{p}}{c_{p}-R}
$$

where $c_{p}$ is the specific heat at constant pressure and $R$ the specific gas constant.

The non-dimensional conservation equations given by Amecke \& Šafař́k can be expressed as a system of non-linear equations as follows:

Conservation of mass:

$$
\frac{K_{2}}{K_{1}} \frac{P_{o 2}}{P_{o 1}} \sqrt{\frac{T_{o 1}}{T_{o 2}}} \Theta_{2} \cos \alpha_{2}-I_{m}=0
$$

where

$$
I_{m}=\int_{0}^{1} \frac{K_{2 y}}{K_{1}} \frac{P_{o 2 y}}{P_{o 1}} \sqrt{\frac{T_{o 1}}{T_{o 2 y}}} \Theta_{2 y} \cos \alpha_{2 y} d(y / s)
$$


Conservation of axial momentum:

$$
\frac{P_{o 2}}{P_{o 1}}\left(2 \frac{q_{2}}{P_{o 2}} \cos ^{2} \alpha_{2}+\frac{P_{2}}{P_{o 2}}\right)-I_{x}=0
$$

where

$$
I_{x}=\int_{0}^{1} \frac{P_{o 2 y}}{P_{o 1}}\left(2 \frac{q_{2 y}}{P_{o 2 y}} \cos ^{2} \alpha_{2 y}+\frac{P_{2 y}}{P_{o 2 y}}\right) d(y / s)
$$

Conservation of tangential momentum:

$$
\frac{P_{o 2}}{P_{o 1}}\left(2 \frac{q_{2}}{P_{o 2}} \cos \alpha_{2} \sin \alpha_{2}\right)-I_{y}=0
$$

where

$$
I_{y}=\int_{0}^{1} \frac{P_{o 2 y}}{P_{o 1}}\left(2 \frac{q_{2 y}}{P_{o 2 y}} \cos \alpha_{2 y} \sin \alpha_{2 y}\right) d(y / s)
$$

Conservation of energy:

$$
\frac{c_{p 2}}{c_{p 1}} \frac{K_{2}}{K_{1}} \frac{P_{o 2}}{P_{o 1}} \sqrt{\frac{T_{o 2}}{T_{o 1}}} \Theta_{2} \cos \alpha_{2}-I_{e}=0
$$

where

$$
I_{e}=\int_{0}^{1} \frac{c_{p 2 y}}{c_{p 1}} \frac{K_{2 y}}{K_{1}} \frac{P_{o 2 y}}{P_{o 1}} \sqrt{\frac{T_{o 2 y}}{T_{o 1}}} \Theta_{2 y} \cos \alpha_{2 y} d(y / s)
$$

Furthermore, the non-dimensional mass flow rate at the measurement plane $\Theta_{2 y}$ can be expressed as,

$$
\Theta_{2 y}=\left(\frac{P_{2 y}}{P_{o 2 y}}\right)^{\frac{1}{\gamma_{2 y}}}\left[\frac{2}{\gamma_{2 y}-1}\left(\frac{\gamma_{2 y}+1}{2}\right)^{\frac{\gamma_{2 y}+1}{\gamma_{2 y}-1}}\right]^{\frac{1}{2}}\left[1-\left(\frac{P_{2 y}}{P_{o 2 y}}\right)^{\frac{\gamma_{2 y}-1}{\gamma_{2 y}}}\right]^{\frac{1}{2}}
$$

the non-dimensional dynamic pressure at the measurement plane is,

$$
\frac{q_{2 y}}{P_{o 2 y}}=\frac{\gamma_{2 y}}{\gamma_{2 y}-1}\left(\frac{P_{2 y}}{P_{o 2 y}}\right)^{\frac{1}{\gamma_{2 y}}}\left[1-\left(\frac{P_{2 y}}{P_{o 2 y}}\right)^{\frac{\gamma_{2 y}-1}{\gamma_{2 y}}}\right]
$$

and $K$, which for the current study is assumed to be constant from the inlet of the cascade to the outlet, is defined as,

$$
K=\sqrt{\frac{\gamma}{R}\left(\frac{2}{\gamma+1}\right)^{\frac{\gamma+1}{\gamma-1}}}
$$

Therefore, the integrals $I_{m}, I_{x}, I_{y}$, and $I_{e}$ defined in Equations B.2b, B.3b, B.4b, and B.5b are functions only of measured quantities. Furthermore, the mixed out total temperature, 
$T_{o 2}$, can be calculated simply using Equations B.2b and B.5b as follows:

$$
T_{o 2}=T_{o 1} \frac{c_{p 1}}{c_{p 2}} \frac{I_{e}}{I_{m}}
$$

However, for the current study the total temperature is constant.

With the temperature determined, the system of equations is reduced to just three equations, namely Equations B.2a, B.3a, and B.4a, with just three unknowns: the mixed-out values of the total pressure $P_{o 2}$, static pressure $P_{2}$, and flow angle $\alpha_{2}$. To simplify the solution to this system of equations, Amecke \& Šafařik express the non-dimensional mixed-out values of the mass flow rate $\Theta_{2}$, dynamic pressure $q_{2} / P_{o 2}$, and static pressure $p_{2} / P_{o 2}$, in terms of the critical Mach number $M_{2}^{*}$ as follows:

$$
\begin{gathered}
\Theta_{2}=\left(1-\frac{\gamma_{2}-1}{\gamma_{2}+1} M_{2}^{* 2}\right)^{\frac{1}{\gamma_{2}-1}}\left(\frac{\gamma_{2}+1}{2}\right)^{\frac{1}{\gamma_{2}-1}} M_{2}^{* 2} \\
\frac{q_{2}}{P_{o 2}}=\left(1-\frac{\gamma_{2}-1}{\gamma_{2}+1} M_{2}^{* 2}\right)^{\frac{1}{\gamma-1}}\left(\frac{\gamma_{2}}{\gamma_{2}+1}\right) M_{2}^{* 2} \\
\frac{P_{2}}{P_{o 2}}=\left(1-\frac{\gamma_{2}-1}{\gamma_{2}+1} M_{2}^{* 2}\right)^{\frac{1}{\gamma-1}}\left(1-\frac{\gamma_{2}-1}{\gamma_{2}+1} M_{2}^{* 2}\right)
\end{gathered}
$$

As shown by Amecke \& Šafař́k (1995), the system of equations can be reduced to a quadratic equation in terms of $M_{2}^{*}$. The two solutions to the resulting quadratic equation are given by,

$$
\begin{aligned}
M_{2}^{* 2}=\left(\frac{\gamma_{2}+1}{2}\right)^{\frac{2}{\gamma_{2}-1}} \frac{I_{x}^{2}}{I_{m}^{* 2}} \times \\
{\left[\frac{1}{2}-\left(\frac{2}{\gamma_{2}+1}\right)^{\frac{2}{\gamma^{-1}}} \frac{I_{m}^{* 2}}{I_{x}^{2}}+\frac{\gamma_{2}+1}{2 \gamma_{2}} \frac{I_{y}^{2}}{I_{x}^{2}} \pm \sqrt{\left.\frac{1}{4}-\left(\frac{2}{\gamma_{2}+1}\right)^{\frac{2}{\gamma_{2}-1}} \frac{I_{m}^{* 2}}{I_{x}^{2}}+\frac{\gamma_{2}^{2}-1}{4 \gamma_{2}^{2}} \frac{I_{y}^{2}}{I_{x}^{2}}\right]}\right.}
\end{aligned}
$$

where,

$$
I_{m}^{*}=I_{m} \frac{K_{1}}{K_{2}} \sqrt{\frac{T_{o 2}}{T_{o 1}}}=\frac{P_{o 2}}{P_{o 1}} \Theta_{2} \cos \alpha_{2}
$$

If limit loading is not reached, the solution will be the minimum of the two positive solutions to Equation B.13. Amecke \& Šafař́k further discuss the interpretation of the solutions to Equation B.13. The mixed-out flow angle can then be calculated from the following equation:

$$
\alpha_{2}=\sin ^{-1}\left[\frac{1}{\gamma_{2} M_{2}^{*}}\left(\frac{\gamma_{2}+1}{2}\right)^{\frac{\gamma_{2}}{\gamma_{2}-1}} \frac{I_{y}}{I_{m}^{*}}\right]
$$


Equations B.10, B.11, and B.12 can also be solved. Finally, from EquationB.14, the mixed-out value of the total pressure $P_{o 2}$ can then be determined. 


\section{Appendix C}

\section{Mixed-Out Results for the Siemens Cascades}

Table C.1: E1S50 Mixed-Out Results

\begin{tabular}{|c|c|c|c|c|c|}
\hline $\boldsymbol{\alpha}_{\mathbf{1}}$ & $\boldsymbol{M}_{\mathbf{2}}$ & $\boldsymbol{R \boldsymbol { R } _ { \mathbf { 2 } } ( \times \mathbf { 1 0 } ^ { - \mathbf { 3 } } )}$ & $\boldsymbol{M}_{\mathbf{1}}$ & $\boldsymbol{\alpha}_{\mathbf{2}}$ & $\boldsymbol{Y}$ \\
\hline \hline $1.0^{\circ}$ & 0.92 & 852 & 0.31 & $61.1^{\circ}$ & 0.0322 \\
\hline $1.0^{\circ}$ & 0.97 & 904 & 0.31 & $61.3^{\circ}$ & 0.0294 \\
\hline $1.0^{\circ}$ & 1.02 & 942 & 0.32 & $61.3^{\circ}$ & 0.0310 \\
\hline $1.0^{\circ}$ & 1.03 & 1002 & 0.32 & $61.1^{\circ}$ & 0.0294 \\
\hline $1.0^{\circ}$ & 1.05 & 1019 & 0.32 & $61.1^{\circ}$ & 0.0360 \\
\hline $1.0^{\circ}$ & 1.12 & 1080 & 0.32 & $60.5^{\circ}$ & 0.0322 \\
\hline $1.0^{\circ}$ & 1.21 & 1177 & 0.32 & $59.4^{\circ}$ & 0.0367 \\
\hline $1.0^{\circ}$ & 1.23 & 1211 & 0.32 & $59.8^{\circ}$ & 0.0361 \\
\hline $1.0^{\circ}$ & 1.37 & 1282 & 0.32 & $57.5^{\circ}$ & 0.0362 \\
\hline $1.0^{\circ}$ & 1.41 & 1397 & 0.32 & $56.8^{\circ}$ & 0.0421 \\
\hline \hline $6.0^{\circ}$ & 0.87 & 817 & 0.30 & $59.1^{\circ}$ & 0.0341 \\
\hline $6.0^{\circ}$ & 0.95 & 854 & 0.31 & $59.7^{\circ}$ & 0.0329 \\
\hline $6.0^{\circ}$ & 1.00 & 915 & 0.31 & $59.8^{\circ}$ & 0.0316 \\
\hline $6.0^{\circ}$ & 1.02 & 968 & 0.31 & $59.8^{\circ}$ & 0.0307 \\
\hline $6.0^{\circ}$ & 1.06 & 1000 & 0.32 & $59.8^{\circ}$ & 0.0346 \\
\hline $6.0^{\circ}$ & 1.11 & 1057 & 0.29 & $59.4^{\circ}$ & 0.0326 \\
\hline
\end{tabular}


Table C.1: E1S50 Mixed-Out Results

\begin{tabular}{|c|c|c|c|c|c|}
\hline$\alpha_{1}$ & $M_{2}$ & $\operatorname{Re}_{2}\left(\times 10^{-3}\right)$ & $M_{1}$ & $\alpha_{2}$ & $Y$ \\
\hline $6.0^{\circ}$ & 1.20 & 1132 & 0.30 & $58.4^{\circ}$ & 0.0350 \\
\hline $6.0^{\circ}$ & 1.30 & 1157 & 0.33 & $58.0^{\circ}$ & 0.0348 \\
\hline $6.0^{\circ}$ & 1.33 & 1222 & 0.34 & $57.1^{\circ}$ & 0.0382 \\
\hline $6.0^{\circ}$ & 1.41 & 1250 & 0.34 & $56.6^{\circ}$ & 0.0273 \\
\hline $15.0^{\circ}$ & 0.91 & 817 & 0.33 & $60.5^{\circ}$ & 0.0387 \\
\hline $15.0^{\circ}$ & 0.96 & 859 & 0.33 & $60.9^{\circ}$ & 0.0357 \\
\hline $15.0^{\circ}$ & 1.03 & 928 & 0.33 & $61.0^{\circ}$ & 0.0332 \\
\hline $15.0^{\circ}$ & 1.06 & 991 & 0.33 & $60.9^{\circ}$ & 0.0330 \\
\hline $15.0^{\circ}$ & 1.07 & 1001 & 0.33 & $60.8^{\circ}$ & 0.0362 \\
\hline $15.0^{\circ}$ & 1.12 & 1054 & 0.33 & $60.5^{\circ}$ & 0.0354 \\
\hline $15.0^{\circ}$ & 1.23 & 1158 & 0.33 & $59.1^{\circ}$ & 0.0378 \\
\hline $15.0^{\circ}$ & 1.26 & 1176 & 0.33 & $59.5^{\circ}$ & 0.0410 \\
\hline $15.0^{\circ}$ & 1.34 & 1242 & 0.33 & $57.5^{\circ}$ & 0.0472 \\
\hline $15.0^{\circ}$ & 1.35 & 1281 & 0.33 & $57.8^{\circ}$ & 0.0487 \\
\hline $25.0^{\circ}$ & 0.87 & 788 & 0.35 & $58.9^{\circ}$ & 0.0458 \\
\hline $25.0^{\circ}$ & 0.93 & 839 & 0.36 & $59.5^{\circ}$ & 0.0477 \\
\hline $25.0^{\circ}$ & 1.00 & 892 & 0.35 & $60.1^{\circ}$ & 0.0404 \\
\hline $25.0^{\circ}$ & 1.06 & 975 & 0.35 & $60.1^{\circ}$ & 0.0393 \\
\hline $25.0^{\circ}$ & 1.07 & 1003 & 0.36 & $60.0^{\circ}$ & 0.0421 \\
\hline $25.0^{\circ}$ & 1.11 & 1042 & 0.36 & $59.8^{\circ}$ & 0.0439 \\
\hline $25.0^{\circ}$ & 1.22 & 1153 & 0.36 & $58.5^{\circ}$ & 0.0440 \\
\hline $25.0^{\circ}$ & 1.25 & 1187 & 0.36 & $58.9^{\circ}$ & 0.0463 \\
\hline $25.0^{\circ}$ & 1.36 & 1302 & 0.35 & $57.2^{\circ}$ & 0.0385 \\
\hline
\end{tabular}


Table C.2: E2S50 Mixed-Out Results

\begin{tabular}{|c|c|c|c|c|c|}
\hline$\alpha_{1}$ & $M_{2}$ & $\operatorname{Re}_{2}\left(\times 10^{-3}\right)$ & $M_{1}$ & $\alpha_{2}$ & $Y$ \\
\hline $9.0^{\circ}$ & 0.85 & 854 & 0.42 & $47.1^{\circ}$ & 0.0299 \\
\hline $9.0^{\circ}$ & 0.91 & 910 & 0.42 & $49.0^{\circ}$ & 0.0300 \\
\hline $9.0^{\circ}$ & 0.94 & 961 & 0.42 & $48.3^{\circ}$ & 0.0296 \\
\hline $9.0^{\circ}$ & 1.00 & 1012 & 0.42 & $49.4^{\circ}$ & 0.0318 \\
\hline $9.0^{\circ}$ & 1.03 & 1077 & 0.41 & $48.3^{\circ}$ & 0.0419 \\
\hline $9.0^{\circ}$ & 1.08 & 1134 & 0.42 & $48.7^{\circ}$ & 0.0482 \\
\hline $9.0^{\circ}$ & 1.15 & 1297 & 0.41 & $47.2^{\circ}$ & 0.0524 \\
\hline $9.0^{\circ}$ & 1.16 & 1333 & 0.41 & $47.6^{\circ}$ & 0.0551 \\
\hline $14.0^{\circ}$ & 0.92 & 914 & 0.43 & $49.1^{\circ}$ & 0.0295 \\
\hline $14.0^{\circ}$ & 1.02 & 1034 & 0.43 & $49.6^{\circ}$ & 0.0302 \\
\hline $14.0^{\circ}$ & 1.10 & 1180 & 0.42 & $49.0^{\circ}$ & 0.0449 \\
\hline $14.0^{\circ}$ & 1.18 & 1371 & 0.42 & $47.9^{\circ}$ & 0.0500 \\
\hline $19.0^{\circ}$ & 0.94 & 954 & 0.45 & $48.3^{\circ}$ & 0.0260 \\
\hline $19.0^{\circ}$ & 1.02 & 1061 & 0.45 & $48.7^{\circ}$ & 0.0278 \\
\hline $19.0^{\circ}$ & 1.11 & 1201 & 0.44 & $48.1^{\circ}$ & 0.0435 \\
\hline $19.0^{\circ}$ & 1.16 & 1397 & 0.43 & $46.9^{\circ}$ & 0.0521 \\
\hline $24.0^{\circ}$ & 0.93 & 940 & 0.47 & $48.8^{\circ}$ & 0.0289 \\
\hline $24.0^{\circ}$ & 1.01 & 1051 & 0.46 & $49.2^{\circ}$ & 0.0316 \\
\hline $24.0^{\circ}$ & 1.09 & 1180 & 0.46 & $48.6^{\circ}$ & 0.0431 \\
\hline $24.0^{\circ}$ & 1.19 & 1346 & 0.45 & $47.3^{\circ}$ & 0.0483 \\
\hline $29.0^{\circ}$ & 0.92 & 909 & 0.49 & $48.2^{\circ}$ & 0.0392 \\
\hline $29.0^{\circ}$ & 1.00 & 1027 & 0.49 & $48.8^{\circ}$ & 0.0378 \\
\hline $29.0^{\circ}$ & 1.09 & 1163 & 0.48 & $48.2^{\circ}$ & 0.0479 \\
\hline $29.0^{\circ}$ & 1.17 & 1380 & 0.48 & $46.9^{\circ}$ & 0.0618 \\
\hline
\end{tabular}


Table C.3: E2S90 Mixed-Out Results

\begin{tabular}{|c|c|c|c|c|c|}
\hline $\boldsymbol{\alpha}_{\mathbf{1}}$ & $\boldsymbol{M}_{\mathbf{2}}$ & $\boldsymbol{R e}_{\mathbf{2}}\left(\times \mathbf{1 0}^{-\mathbf{3}}\right)$ & $\boldsymbol{M}_{\mathbf{1}}$ & $\boldsymbol{\alpha}_{\mathbf{2}}$ & $\boldsymbol{Y}$ \\
\hline \hline$-12.0^{\circ}$ & 0.86 & 692 & 0.36 & $55.2^{\circ}$ & 0.0374 \\
\hline$-12.0^{\circ}$ & 0.96 & 784 & 0.37 & $56.1^{\circ}$ & 0.0294 \\
\hline$-12.0^{\circ}$ & 1.09 & 894 & 0.37 & $55.5^{\circ}$ & 0.0338 \\
\hline$-12.0^{\circ}$ & 1.18 & 975 & 0.37 & $54.4^{\circ}$ & 0.0407 \\
\hline \hline$-20.0^{\circ}$ & 0.87 & 703 & 0.39 & $55.7^{\circ}$ & 0.0293 \\
\hline$-20.0^{\circ}$ & 0.97 & 801 & 0.39 & $56.5^{\circ}$ & 0.0231 \\
\hline$-20.0^{\circ}$ & 1.09 & 924 & 0.40 & $55.7^{\circ}$ & 0.0306 \\
\hline$-20.0^{\circ}$ & 1.16 & 1026 & 0.40 & $54.6^{\circ}$ & 0.0405 \\
\hline \hline$-30.0^{\circ}$ & 0.88 & 719 & 0.43 & $55.5^{\circ}$ & 0.0240 \\
\hline$-30.0^{\circ}$ & 0.99 & 813 & 0.43 & $56.2^{\circ}$ & 0.0210 \\
\hline$-30.0^{\circ}$ & 1.10 & 926 & 0.44 & $55.4^{\circ}$ & 0.0277 \\
\hline$-30.0^{\circ}$ & 1.17 & 982 & 0.44 & $54.6^{\circ}$ & 0.0373 \\
\hline
\end{tabular}

